\title{
Untersuchungen zum Lumineszenzverhalten des Aluminiumnitrids und der Aufbau einer Kurzzeit-Lumineszenz-Spektroskopie- Apparatur
}

\author{
Dissertation \\ zur Erlangung des mathematisch-naturwissenschaftlichen Doktorgrades \\ "Doctor rerum naturalium" \\ der Georg-August-Universität Göttingen \\ im Promotionsprogramm ProPhys \\ der Georg-August University School of Science (GAUSS)
}

vorgelegt von

TRISTAN KOPPE

aus Mühlhausen Thomas-Müntzer-Stadt 


\section{$\underline{\text { Betreuungsausschuss }}$}

Professor Dr. Hans Christian Hofsäss - II. Physikalisches Institut - Universität Göttingen

Dr. Ulrich Vetter - II. Physikalisches Institut - Universität Göttingen

\section{Mitglieder der Prüfungskommission}

Referent: Professor Dr. Hans Christian Hofsäss - II. Physikalisches Institut - Universität Göttingen

Korreferent: Professor Dr. Michael Seibt - IV. Physikalisches Institut - Universität Göttingen

\section{Weitere Mitglieder der Prüfungskommission}

Professor Dr. Wolfram Kollatschny - Institut für Astrophysik - Universität Göttingen

Professor Dr. Vasily Moshnyaga - I. Physikalisches Institut - Universität Göttingen

Professor Dr. Andreas Tilgner - Institut für Geophysik und Fluiddynamik - Universität Göttingen

Professor Dr. Angela Rizzi - IV. Physikalisches Institut - Universität Göttingen

Tag der mündlichen Prüfung: 05.07.2017 


\section{Inhaltsverzeichnis}

$\begin{array}{lll}1 & \text { Einleitung } & 1\end{array}$

2 Lumineszenz in Aluminiumnitrid 5

2.1 Bandkantenlumineszenz $\ldots \ldots \ldots \ldots \ldots$

2.1 .1 Bandstruktur . . . . . . . . . . . . . . . . 6

$2.1 .2 \quad$ Freie Exzitonen . . . . . . . . . . . . . . . . . . . . . . 8

2.1 .3 Gebundene Exzitonen . . . . . . . . . . . . . . . . . . 12

$2.1 .4 \quad$ XX, P-Band und EHP . . . . . . . . . . . . . . . 17

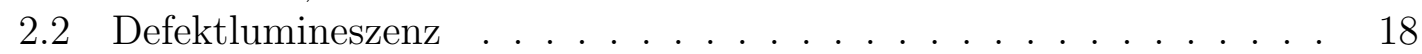

2.2 .1 Intrinsische Defekte . . . . . . . . . . . . . . . . . . . . 23

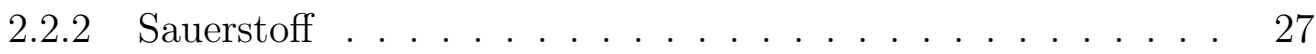

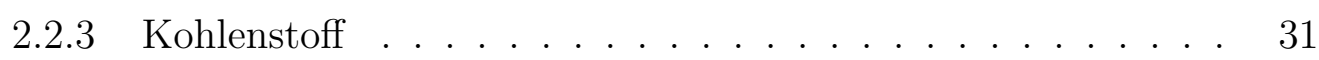

2.2 .4 Metalle . . . . . . . . . . . . . . . . . . . . 33

$2.2 .5 \quad$ Akzeptoren und Donatoren $\ldots \ldots \ldots \ldots$

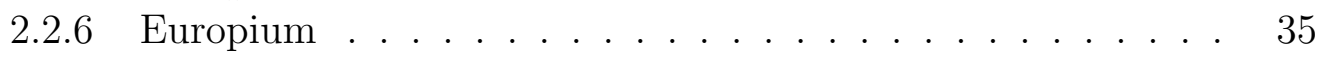

\begin{tabular}{|lll}
3 & Experimenteller Aufbau & 39
\end{tabular}

3.1 Lasersystem $\ldots \ldots \ldots \ldots \ldots \ldots$. . . . . . . . . . . . . . 40

3.1 .1 Pumplaser . . . . . . . . . . . . . . . . . . . . . . . . . 40

$\begin{array}{llll}3.1 .2 & \text { Titan-Saphir Laser } \ldots \ldots \ldots & \ldots\end{array}$

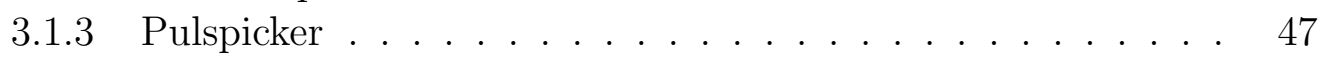

3.1 .4 Harmonischer Generator . . . . . . . . . . . . . . . . . 49

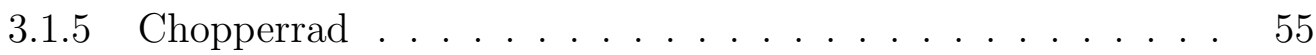

$3.1 .6 \quad$ Fokussiereinheit . . . . . . . . . . . . . . . . . . . 58

3.2 Probenkammer . . . . . . . . . . . . . . . . . . . . . . . . . . 5

3.3 Optisches Messsystem . . . . . . . . . . . . . . . . . . . . . . . . . . 62

$3.3 .1 \quad$ Spektrometer . . . . . . . . . . . . . . . . . . . . . 63

3.3 .2 Streak-Kamera . . . . . . . . . . . . . . . . . 68

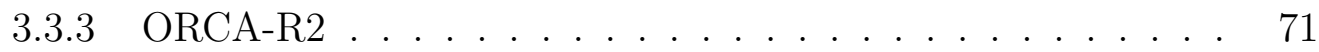

3.3 .4 Messverfahren . . . . . . . . . . . . . . . . . . . . . 74

3.3 .5 Arbeitsmodi . . . . . . . . . . . . . . . . . 78

4 Datenanalyse 83

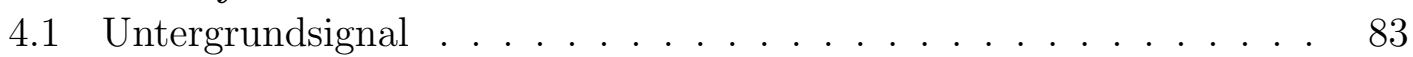

4.2 Sensitivität $\ldots \ldots \ldots \ldots \ldots \ldots \ldots$. . . . . . . . . . . . . 84 
4.3 Zeitkorrektur . . . . . . . . . . . . . . . . . . 86

4.4 Krümmungskorrektur . . . . . . . . . . . . . . . . . . . . . . . . . 89

4.5 Verkippung $\ldots \ldots \ldots \ldots$. . . . . . . . . . . . . . . . . . . . . . . . 93

4.6 Erweiterte Zeitfenster . . . . . . . . . . . . . . . . . . . . . . . . . . . . . . 94

4.7 Ratengleichungen . . . . . . . . . . . . . . . . . . . . . . . 95

$\begin{array}{lll}5 & \text { Experimentelle Untersuchung von Aluminiumnitrid } & 101\end{array}$

5.1 Proben . . . . . . . . . . . . . . . . . 101

5.2 Defektlumineszenz . . . . . . . . . . . . . . . . . 103

5.2 .1 Statisches Lumineszenzverhalten. . . . . . . . . . . . . . . . 103

5.2 .2 Zeitabhängiges Lumineszenzverhalten . . . . . . . . . . . . . 112

$5.2 .3 \quad$ Diskussion und Zuordnung . . . . . . . . . . . . . . . . . . . 120

5.3 Mangan-spezifische Lumineszenz . . . . . . . . . . . . . . . . . . . . 121

5.3 .1 Beschreibung der Unterstruktur . . . . . . . . . . . . . . . . 122

$5.3 .2 \quad$ Lebensdauer . . . . . . . . . . . . . . . . . . . . . . . . 130

5.3 .3 Diskussion . . . . . . . . . . . . . . . . . . . 135

$5.4 \quad$ Europium-spezifische Lumineszenz . . . . . . . . . . . . . . . . . . . 135

5.4 .1 Statisches Lumineszenzverhalten um $1.987 \mathrm{eV}$. . . . . . . . 136

5.4 .2 Zeitabhängiges Lumineszenzverhalten . . . . . . . . . . . . . 137

5.4 .3 Diskussion . . . . . . . . . . . . . . . . . . . . . . . . 142

$\begin{array}{lll}6 & \text { Zusammenfassung und Ausblick } & 145\end{array}$

\begin{tabular}{lr}
\hline Literaturverzeichnis & 150
\end{tabular}

\begin{tabular}{ll}
\hline A Anhang & 181
\end{tabular} 


\section{Einleitung}

Die Entdeckung des Aluminiumnitrid (AlN) ist bis zu einem Bericht von Briegleb und Geuther [1] ausgehend von Experimenten durchgeführt in Göttingen aus dem Jahre 1862 zurück zu verfolgen. Sie präparierten dabei Feilspäne aus Aluminium in einem Porzellanschiffchen und heizten dieses unter einer Stickstoffatmosphäre für etwa zwei Stunden auf. Nach der Abkühlung der Präparate beobachteten sie eine signifikante Gewichtszunahme einhergehend mit einer weißlichen Oberfläche. Das Innere der Späne zeigte zudem eine matte gelb-bräunliche Verfärbung. Zusätzlich fanden sie auch Hinweise darauf, dass sich in dem Material nun Stickstoff befinden könnte. Unabhängig von Briegleb und Geuther präparierte 1876 Mallet [2,3] AlN. Ursprünglich wollte er Kohlenstoff in Aluminium ähnlich wie schon bei Eisen einbringen; dieser Versuch misslang. Jedoch konnte er das stöchiometrische Verhältnis von Aluminium zu Stickstoff in seinen Proben auf Eins bestimmen. Eine frühe sehr präzise kristallographische Untersuchung von AlN wurde von Ott [4] an Präparaten, welche nach dem sogenannten Serpek-Prozess [5] hergestellt wurden, unternommen. Dabei fand er in seinen Proben eine wurtzitische Kristallstruktur mit einem axialen Verhältnis von $c / a=1.60_{1}$ und einen internen Parameter von $u=0.380_{1}$ unter der Nutzung der Laue-Methode vor (die tiefgestellten Zahlen sind eine zusätzliche Stelle, welche jedoch als unsicher gilt).

Aufgrund seiner hohen thermischen Leitfähigkeit $\left(320 \mathrm{Wm}^{-1} \mathrm{~K}^{-1}\right.$ [6, 7] $)$ in Verbindung mit seiner gleichzeitigen hohen intrinsischen elektrischen Isolationsfähigkeit $\left(10^{11} \Omega \mathrm{cm}-10^{13} \Omega \mathrm{cm}[8]\right)$ und einem zu Silizium sehr ähnlichen thermischen Ausdehnungskoeffizient $\left(2.7 \times 10^{-6} \mathrm{~K}^{-1}\right.$ bei Raumtemperatur [9]) findet AlN heutzutage eine breite Anwendung als Material für Kühlkörper in Hochleistungselektronik. Außerdem eignet es sich infolge seiner sehr hohen Schallgeschwindigkeiten $\left(v_{L}=10127 \mathrm{~ms}^{-1}\right.$ und $\left.v_{S}=6333 \mathrm{~ms}^{-1}[10]\right)$ und piezoelektrischen Eigenschaften zur Nutzung in Baugruppen mit akustischen Bulk- sowie auch Oberflächenwellen als Trägermaterial; insbesondere für Bauelemente, die im Frequenzbereich mehrerer $\mathrm{GHz}$ operieren [11, 12. Des weiteren können in rein elektronischen Anwendungen GaN/AlN-Heterostrukturen als eine Basis für high-electron-mobility Transistoren (HEMT) dienen [13, 14]. Überdies findet AlN auch in Gebieten der Optik einen Einsatz. In frühen Tagen wurde es zunächst als Wirt für ein Luminophor, dotiert mit Silizium [15] oder auch mit Mangan [16], eingesetzt. Später folgten testweise Anwendungsversuche als UV-Detektor mit einer Unempfindlichkeit gegenüber sichtbarem Licht [17], sowie die Entwicklung von LEDs mit Wellenlängen bis zu einem Bereich von $210 \mathrm{~nm}$ [18, 19]. Aufgrund seiner großen Bandlücke eignet sich AlN ideal als 
Wirtsgitter für Untersuchungen von optischen Emissionen der Elemente der seltenen Erden bis in den UV-Bereich hinein; beispielsweise für $G d^{3+}[20]$. Bis zum heutigen Tag kann AlN jedoch nicht in Anwendungen, in denen eine hohe effiziente Dotierung mit n-Typ- als auch p-Typ-Leitfähigkeit benötigt wird, ohne weiteres eingesetzt werden. Infolge unzureichender Kristallqualitäten können der Dotierung als auch der thermische Leitfähigkeit entgegenstehende kompensierende Effekte auftreten, die durch ein unbeabsichtigtes Einbringen verschiedener Störstellen herrühren [6, 7]. Im Allgemeinen scheint das Erreichen einer effizienten p-Typ-Leitfähigkeit bei Raumtemperatur in Bulk-AlN ein großes Problem darzustellen [21,22].

Um diese Eigenschaft der unzureichenden elektrischen Leitfähigkeit besser nachvollziehen und eine Verbesserung der Situation erreichen zu können, ist ein genaues Verständnis der energetischen Positionen der unterschiedlichen möglichen Donatoren als auch Akzeptoren vonnöten. Des weiteren müssen die während der Herstellung oft unwillkürlich eingebrachten Störstellen spezifiziert werden, um eine zielgerichtete Verbesserung der Kristallqualität erreichen zu können. Da viele der Defekte auch in optischen Rekombinationen wiedergefunden werden können, erweist sich eine spektroskopische Untersuchung des Materialsystems als eine Möglichkeit der Einschränkung der verschiedenen Ursachen. So kann im Idealfall aufgrund der energetischen Position gegebenenfalls auch gekoppelt mit dem zeitlichen Verhalten eines beobachteten Lumineszenzsignals ein eindeutiger Rückschluss auf dessen Ursache getroffen werden. In Kombination mit unterschiedlichen experimentellen Bedingungen während der Herstellung können so eine sich beispielsweise ändernde Konzentration von Leerstellen oder Einschlüssen von Fremdatomen verfolgt werden. Hierfür bedarf es jedoch einer Kenntnis der möglichen Ursachen mit deren spektroskopischen als auch elektrischen Eigenschaften. In der Literatur existiert eine Vielzahl an Publikationen, die sich mit diesen Eigenschaften sowohl theoretisch als auch experimentell auseinandersetzen. Dennoch gab es aber bis dato keine übersichtliche Zusammenfassung jener, was nun unter anderem im Rahmen dieser Arbeit nachgeholt wurde und als Basis für zukünftige Analysen genutzt werden kann.

\section{Gliederung und Inhalt der Arbeit}

Die hier präsentierte Arbeit gliedert sich in drei große Bereiche:

Zunächst wird basierend auf einer umfangreichen Literaturrecherche auf die in AlN bis jetzt beobachteten Lumineszenzen nahe der Bandkante als auch energetisch inmitten der Bandlücke befindlichen optischen Übergänge ausführlich eingegangen. Schon vorher existierten zwar einige Zusammenfassungen der Lumineszenzen in AlN, jedoch wurden dort entweder nur frühere eigene Arbeiten oder nur ein kleiner Ausschnitt der dazu verfügbaren Literatur berücksichtigt 23 28]. In Kapitel 2 wird eine Zusammenfassung der in AlN bis jetzt beobachteten optischen Transfers gegeben, wobei sich dabei auf die wurtzitische Phase (üblicherweise hergestellt) von Bulk-AlN beschränkt wird. Explizit schließt dies Nanopulver, Nanostäbchen 
als auch Lumineszenzen in Verbindung mit Quantentöpfen oder Quantenpunkten aus. Bis auf die Lumineszenz des Europium werden auch keine weiteren optischen Rekombinationen, welche anderen Elementen der seltenen Erden zugeschrieben werden, betrachtet.

Der zweite Teil (Kapitel 3) befasst sich mit der Inbetriebnahme und Charakterisierung eines Kurzzeit-Lumineszenz-Spektroskopie-Aufbaus zur Untersuchung von Festkörpern sowie Flüssigkeiten vom nahen UV- über den sichtbaren bis hin zum nahen Infrarotbereich. Dabei werden die wichtigsten Leistungsparameter der einzelnen Komponenten und die maximale Effizienz des Gesamtsystems bestimmt. Zudem werden in Kapitel 4 die verschiedenen Schritte für eine Aufarbeitung der vorhandenen Messdaten zur Erzielung eines möglichst hohen Informationsgewinns dargestellt.

Im dritten Teil (Kapitel 5) wird das vorher charakterisierte System zur spektroskopischen Untersuchung von AlN experimentell angewendet. Bei den Präparaten handelt es sich um AlN, welches nach der Hochdruck-Hochtemperatur-Methode hergestellt wurde. Einhergehend mit einer ausführlichen Analyse der Messdaten und einer Zuordnung der beobachteten Lumineszenzen werden die Stärken dieses Aufbaus sowie dessen Anwendungsgrenzen deutlich. 



\section{Lumineszenz in Aluminiumnitrid}

In der Literatur gibt es eine Vielzahl von experimentellen, sowie auch theoretischen Untersuchungen zur Bandstruktur von AlN, sowie der energetischen Position von Defekten oder potentiellen Akzeptoren und Donatoren innerhalb dieser. Als ein sehr hilfreiches Mittel hat sich dabei die Lumineszenz-Spektroskopie erwiesen, mit welcher die energetischen Abstände zwischen verschiedenen angeregten Zuständen untersucht werden können. Eine kurze schematische Darstellung der unterschiedlichen beobachtbaren Übergänge in AlN ist in Abbildung 2.1 gegeben.

Im Rahmen dieser Arbeit wurde nach einer intensiven Literaturrecherche von sowohl theoretischen als auch experimentellen Berichten, welche sich mit dem Lumineszenzverhalten und den dazugehörigen Energieniveaus beschäftigen, ein umfassendes Review zu diesem Thema veröffentlicht [29]. Das folgende Kapitel besteht inhaltlich zu großen Teilen aus dieser Veröffentlichung und soll einen breiten Überblick der Forschung aus den letzten Jahrzehnten bis hin zu aktuellen Ergebnissen liefern. Dabei werden die unter Umständen sehr unterschiedlichen Standpunkte innerhalb der Literatur, beispielsweise bezüglich Defektkomplexen mit Sauerstoff, vorgestellt.

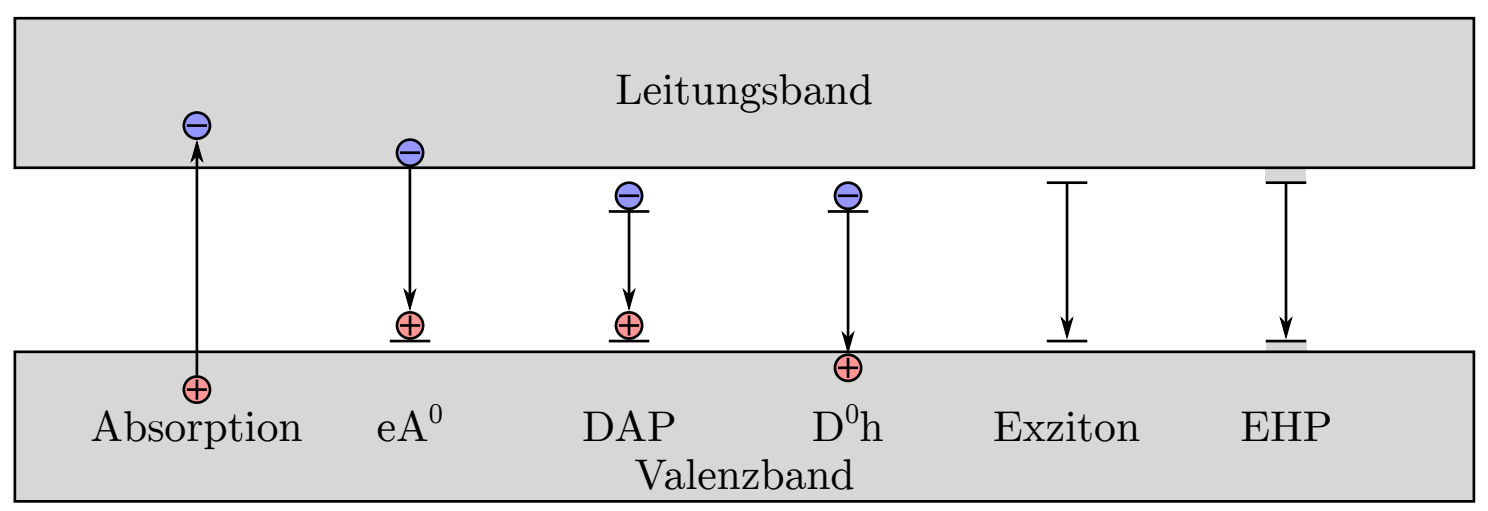

Abbildung 2.1: Schematische Darstellung verschiedener optischer Übergänge, welche in AlN schon beobachtet worden sind. Bei den ersten drei Abregungen handelt es sich um Übergänge mit Energien typischerweise weit unterhalb der Bandkante, während die letzten beiden nahe dieser stattfinden. 


\subsection{Bandkantenlumineszenz}

Aufgrund unzureichender Kristallqualität früher untersuchter AlN-Proben trat ein stark ausgeprägtes Absorptionsband ausgehend vom nahen UV-Bereich auf. Jenes führte zu einer Unterdrückung der bandkantennahen Lumineszenz [30]. Die erste Bandkanten-Lumineszenz in AlN wurde von Hossain et al. im Jahr 1996 beobachtet 31]. Sie verwendeten dafür dünne Filme aus AlN, welche mittels MOCVD auf Saphir und $\mathrm{SiC}$ aufgewachsen und über Kathodolumineszenz vermessen wurden. Später fanden Teofilov et al. Lumineszenz nahe der Bandkante in dünnen, über MBE auf Silizium aufgebrachten AlN-Filmen, ebenfalls untersucht mit einem Kathodolumineszenzaufbau. In den letzten zwei Dekaden wurde eine Vielzahl von experimentellen Untersuchungen, welche sich mit Lumineszenz in AlN nahe der Bandkante beschäftigen, publiziert [19, 21, 22, 31, 81]. Im folgenden Teilabschnitt findet eine genauere Betrachtung jener statt. Zunächst soll jedoch ein kurzer Überblick über die Bandstruktur in AlN gegeben werden.

\subsubsection{Bandstruktur}

Zur Voraussage und auch zur Erklärung physikalischer Eigenschaften von Halbleitern sind neben experimentellen Untersuchungen auch theoretische Simulationen erforderlich. Erste Bandstruktur-Berechnungen von AlN wurden in den späten 1960er und frühen 1970er Jahren zur Bestimmung der Bandlücke und der Form der Valenzbänder durchgeführt 82 85. Diese frühen Untersuchungen ergaben, dass es sich bei AlN um einen Halbleiter mit direkter Bandlücke am $\Gamma$-Punkt handelt. Die theoretisch ermittelte Größe dieser lag bei jenen Kalkulationen im Bereich zwischen $2.35 \mathrm{eV}$ [82 und $5.31 \mathrm{eV}$ [85] (größere Werte von semiempirischen Ansätzen) und ergaben eine negative Kristallfeldaufspaltung zwischen $\Delta_{c f}=-270 \mathrm{meV}$ 85 und $\Delta_{c f}=-70 \mathrm{meV}$ 83. Ein Grund für die sehr unterschiedlichen Ergebnisse bei den ermittelten $\Delta_{c f}$ ist der in den Simulationen gewählte Wert für den inneren Parameter $u$ (Versatz der beiden Untergitter). In einer idealen wurtzitischen Struktur hat dieser Parameter eine Größe von $u=3 / 8=0.375$, während in relaxierten AlN-Systemen, berechnet über eine Minimierung der Gesamtenergie, ein Wert von $u \approx 0.382[86]$ gefunden wurde. Jiang et al. 87] fand einen linearen Abfall von $\Delta_{c f}$ von $\sim 20 \mathrm{meV}$ bei einem Anstieg von $u$ um 0.001. Hingegen führt ein Abfall des strukturellen Seitenverhältnisses $c / a$ (ideal: $c / a=\sqrt{8 / 3}=1.632$, AlN: $c / a \approx 1.601$ [88]) um 0.001 auch zu einer Verringerung von $\Delta_{c f} \mathrm{um} \sim 3 \mathrm{meV}$.

Neben dem Kristallfeld führt auch die Spin-Bahn-Kopplung zu einer weiteren Aufspaltung der Valenzbänder. Im Fall einer quasi-kubischen Näherung [26] startet der niedrigste publizierte Wert bei $\Delta_{s o}=11 \mathrm{meV}$ [89] und erreicht in andernorts veröffentlichten Simulationen eine maximale Größe von bis zu $\Delta_{s o}=21.8 \mathrm{meV}$ [90]. In konsequent wurtzitisch gerechneten Kalkulationen verhalten sich die Parameter 


\begin{tabular}{ccc}
\hline Übergang & $I(E \| c)$ & $I(E \perp c)$ \\
\hline$\Gamma_{7 c} \leftrightarrow \Gamma_{7 \nu}^{+}$ & $b^{2} \frac{m_{0}^{2}}{\hbar^{2}} P_{1}^{2}$ & $a^{2} \frac{m_{0}^{2}}{2 \hbar^{2}} P_{2}^{2}$ \\
$\Gamma_{7 c} \leftrightarrow \Gamma_{9 \nu}$ & 0 & $\frac{m_{0}^{2}}{2 \hbar^{2}} P_{2}^{2}$ \\
$\Gamma_{7 c} \leftrightarrow \Gamma_{7 \nu}^{-}$ & $a^{2} \frac{m_{0}^{2}}{\hbar^{2}} P_{1}^{2}$ & $b^{2} \frac{m_{0}^{2}}{2 \hbar^{2}} P_{2}^{2}$ \\
\hline
\end{tabular}

Tabelle 2.1: Quadratische Übergangsmomente $I$ zwischen dem niedrigsten Leitungsband $\left(\Gamma_{7 c}\right)$ und dem Valenzband-Tripel für Photonen polarisiert entlang $(\|)$ und senkrecht $(\perp)$ der c-Achse nach [93]. Hierbei stehen $P_{1}^{2}$ und $P_{2}^{2}$ für Parameter in Kane's Modell (siehe Gleichung 2.3). Die Vorfaktoren $a^{2}$ und $b^{2}$ sind in Gleichung 2.4 definiert und folgen der direkten Beziehung $a^{2}+b^{2}=1$.

$\Delta_{2,3}$ nahezu identisch, mit Werten zwischen $5.7 \mathrm{meV}$ [91] und $7.5 \mathrm{meV}$ [90], wodurch die Valenzbandanalyse über eine quasi-kubische Näherung eine Rechtfertigung erhält.

Aufgrund der starken negativen Kristallfeldaufspaltung zeigen die Valenzbänder folgende energetische Anordnung: $\Gamma_{7 \nu}^{+}, \Gamma_{9 \nu}, \Gamma_{7 \nu}^{-}[92]$. Die Energie-Level der einzelnen Bänder können nach den Gleichungen 2.1 und 2.2 93 folgendermaßen berechnet werden (Beziehung zu quasi-kubischer Näherung: $\Delta_{1}=\Delta_{c f}, \Delta_{2}=\Delta_{3}=1 / 3 \Delta_{s o}$ ):

$$
\begin{gathered}
E\left(\Gamma_{9 \nu}\right)=\Delta_{1}+\Delta_{2} \\
E\left(\Gamma_{7 \nu}^{ \pm}\right)=\frac{\Delta_{1}-\Delta_{2}}{2} \pm\left[\left(\frac{\Delta_{1}-\Delta_{2}}{2}\right)^{2}+2 \Delta_{3}^{2}\right]^{1 / 2}
\end{gathered}
$$

Während Ergebnisse aus Simulationen von Valenzbändern in guter Übereinstimmung mit experimentellen Studien stehen, treten bei der Voraussage der Bandlücke große Unterschiede zu diesen auf. Häufig basieren die Berechnungen auf einer lokalen Dichtenäherung (LDA) oder einer Gradientennäherung (GGA), welche beide eine erhebliche Unterschätzung der Bandlücke aufgrund der fehlenden nicht lokalisierten Austauschpotentiale aufweisen (siehe Tabelle A.1). Dem entgegen zeigen Methoden wie Hatree-Fock (HF) [94], welche die teilweise Abschirmung dieses nicht lokalen Austauschs unberücksichtigt lassen, eine deutliche Überschätzung. Um die experimentell bestimmte Bandlücke zu erreichen, finden in den letzten Jahren häufig hybride Ansätze, wie zum Beispiel nach dem Schema von Heyd, Scuseria und Ernzerhof (HSE) [95], Anwendung. Diese Methoden besitzen einen einstellbaren Parameter, um eine Bandlücke von beispielsweise $6.1 \mathrm{eV}$ zu erreichen. Im Fall von HSE hat solch ein Mischungsparameter (Mischung zwischen HF und LDA) typischerweise eine Größe von 0.32 87,96 100]. 


\subsubsection{Freie Exzitonen}

Bei tiefen Temperaturen rekombinieren in AlN Elektron-Loch-Paare zumeist erst nach einer vorherigen Bildung von Exzitonen. Aufgrund der dreifachen Valenzbandaufspaltung am $\Gamma$-Punkt besitzt AlN drei verschiedene Arten von freien Exzitonen: $F X_{A}, F X_{B}$ und $F X_{C}$ mit dabei aufsteigender energetischer Reihenfolge. Die in der Literatur in experimentellen Messungen durchschnittlich gefundenen Rekombinationsenergien für $F X_{B}$ und $F X_{C}$ [35, 38 42 liegen jeweils 208(14) meV und $222(15) \mathrm{meV}$ über der des freien Exzitons $F X_{A}$. Diese Ergebnisse decken sich gut mit denen aus vorhergehenden theoretischen Untersuchungen (siehe dazu Tabelle A.1). Die freien Exzitonen $F X_{B}$ und $F X_{C}$ zeigen im Vergleich zu ihrem energetischen Abstand eine zumeist sehr breite Peak-Form. Daher ist eine Unterscheidung bei Messungen mit niedriger Statistik oder vergleichsweise hohem Untergrund nicht immer möglich. Folglich werden diese Peaks deshalb manchmal auch nur als ein ausgeweitetes Signal beschrieben 49 53,81. Als Ergebnis der in AlN relativ großen negativen Kristallfeldaufspaltung führt die Valenzbandanordnung $\Gamma_{7 \nu}^{+}, \Gamma_{9 \nu}, \Gamma_{7 \nu}^{-} \mathrm{zu}$ einer für die meisten Materialien sehr ungewöhnlichen Polarisation der freien Exzitonen. In Tabelle 2.1 sind die quadratischen Übergangsmomente zwischen dem niedrigstem Leitungsband $\left(\Gamma_{7 c}\right)$ und dem Valenzband-Tripel für Licht polarisiert entlang als auch senkrecht der c-Achse des Kristalls gegeben.

$$
\begin{gathered}
P_{1}^{2}=\frac{\hbar^{2}}{2 m_{0}}\left(\frac{m_{0}}{m_{e}^{\perp}}-1\right) \frac{\left(E_{g}+\Delta_{1}+\Delta_{2}\right)\left(E_{g}+2 \Delta_{2}\right)-2 \Delta_{3}^{2}}{E_{g}+2 \Delta_{2}}, \\
P_{2}^{2}=\frac{\hbar^{2}}{2 m_{0}}\left(\frac{m_{0}}{m_{e}^{\|}}-1\right) \frac{E_{g}\left[\left(E_{g}+\Delta_{1}+\Delta_{2}\right)\left(E_{g}+2 \Delta_{2}\right)-2 \Delta_{3}^{2}\right]}{\left(E_{g}+\Delta_{1}+\Delta_{2}\right)\left(E_{g}+\Delta_{2}\right)-\Delta_{3}^{2}} \\
a^{2}=\frac{E\left(\Gamma_{7 \nu}^{+}\right)}{E\left(\Gamma_{7 \nu}^{+}\right)-E\left(\Gamma_{7 \nu}^{-}\right)}, b^{2}=\frac{E\left(\Gamma_{7 \nu}^{-}\right)}{E\left(\Gamma_{7 \nu}^{-}\right)-E\left(\Gamma_{7 \nu}^{+}\right)}
\end{gathered}
$$

Hierbei stehen $P_{1}^{2}$ und $P_{2}^{2}$ für Parameter in Kane's Modell (siehe die Gleichungen 2.3 im Fall für wurtzitische Halbleiter [93, 101] mit Elektronenmassen im Leitungsband $m_{e}^{\perp}$ und $m_{e}^{\|}$, der freien Elektronenmasse $m_{0}$ und der energetischen Bandlücke $E_{g}$. In Tabelle 2.2 sind die quadratischen Übergangsmomente für AlN mit exemplarischen Stoffkonstanten berechnet. Daraus folgt für $F X_{A}$ eine nahezu vollständige Polarisation in die Richtung $E \| c$, während $F X_{B}$ und $F X_{C}$ stark oder im Fall von $F X_{B}$ sogar vollständig in Richtung $E \perp c$ auftreten. Auch dies konnte von verschiedenen Experimenten bestätigt werden [43,59, 102]. Dieser Umstand erklärt auch die unterschiedlichen experimentell, vor allem in früheren Studien oft größeren, gefundenen Werte für die Bandlücke. Jene wurde dort über Absorptions-, Transmissions- oder Reflexionsmessungen bestimmt, wobei die in diesen Fällen untersuchten dünnen Filme mit einer Orientierung in Richtung der c-Achse vorlagen. In einer Konfiguration, bei welcher mit senkrechtem Lichteinfall bezogen zur Oberfläche der Probe gearbeitet wird, hat dies eine Polarisation von $E \perp c$ zur 


\begin{tabular}{ccc}
\hline Übergang & $I(E \| c)$ & $I(E \perp c)$ \\
\hline$\Gamma_{7 c} \leftrightarrow \Gamma_{7 \nu}^{+}$ & 0.4674 & 0.0005 \\
$\Gamma_{7 c} \leftrightarrow \Gamma_{9 \nu}$ & 0 & 0.2658 \\
$\Gamma_{7 c} \leftrightarrow \Gamma_{7 \nu}^{-}$ & 0.0010 & 0.2653 \\
\hline
\end{tabular}

Tabelle 2.2: Quadratische Übergangsmomente $I$ (zusammen auf eins normiert) zwischen dem niedrigstem Leitungsband $\left(\Gamma_{7 c}\right)$ und dem Valenzband-Tripel für Photonen polarisiert entlang $(\|)$ und senkrecht $(\perp)$ der c-Achse. In die in Tabelle 2.1 angegebenen Gleichungen wurden stoffspezifische Werte für AlN eingesetzt: $\Delta_{1}=-200 \mathrm{meV}, \Delta_{2}=\Delta_{3}=6.6 \mathrm{meV}, E_{g}=6.1 \mathrm{eV}, m_{z} / m_{e}=0.32$ [105 und $m_{t} / m_{e}=0.30105$.

Folge, wodurch lediglich eine nahezu exklusive Anregung zwischen $\Gamma_{9 \nu}$ und $\Gamma_{7 \nu}^{-}$mit dem Leitungsband $\Gamma_{7 c}$ stattfindet [103]. Aufgrund dieser Polarisationseigenschaften leidet die klassische c-Ebenen-Konfiguration in AlN an einer starken Limitierung der Effizienz für lichtemittierende Anwendungen, im Gegensatz zum nahestehenden GaN, wo das freie Exziton $F X_{A}$ eine Polarisation in Richtung $E \perp c$ aufweist 104.

Eine genauere Betrachtung bezüglich der Wechselwirkung zwischen den Bändern $\Gamma_{7 c}$ und $\Gamma_{7 \nu}^{+}$zeigt, dass sich im wurtzitischem AlN Exzitonen mit $\Gamma^{1}, \Gamma^{2}$ und $\Gamma^{5}$ Symmetrie (nach [106 jedoch mit vertauschter Definition von $\Gamma^{5}$ und $\Gamma^{6}$ ) ausbilden, wobei bei der letzteren eine zweifache Entartung auftritt:

$$
\Gamma_{c}^{7} \otimes \Gamma_{\nu}^{7} \rightarrow \Gamma^{1}(z)+\Gamma^{2}+\Gamma^{5}(x+y)
$$

Exzitonen mit $\Gamma^{2}$-Symmetrie sind dipolverboten und können dahingehend in direkten optischen Rekombinationsprozessen nicht beobachtet werden. Die beiden Exzitonen nach Art von $\Gamma^{2}$ und $\Gamma^{5}$ bilden sich nur unter der Berücksichtigung der Spin-BahnKopplung und haben im Fall von $\Gamma^{5}$ ein sehr kleines Matrix-Übergangsmoment verglichen zu $\Gamma^{1}$. Dies führt zu der nahezu vollständigen $E \| c$-Polarisation für das freie Exziton $F X_{A}$ in AlN. Aufgrund der Elektron-Loch-Austauschwechselwirkung ergibt sich eine Energiedifferenz von $2 j$ zwischen den beiden Zuständen $\Gamma^{1}$ und $\Gamma^{5}$, wobei hier $j$ die Austauschkonstante darstellt. Das Vorzeichen dieser Konstante steht in der aktuell verfügbaren Literatur noch immer unter Diskussion. Es existieren sowohl experimentelle Ergebnisse, welche ein positives [80, 81, 107, 108] aber auch jene, die ein negatives Vorzeichen [34 37] suggerieren. Dabei fanden Ishii et al. eine Austauschkonstante der Größe $j=6.8 \mathrm{meV}$, welche im Vergleich zu allen anderen typischen III-V oder auch II-VI-Verbindungshalbleitern den größten gefundenen Wert darstellt [107]. Dagegen berichten Feneberg et al. von einem $j=-4.0 \mathrm{meV}$, was noch für keinen anderen Bulk-Halbleiter jemals zuvor beobachtet wurde [35]. Diese jeweiligen experimentell gefundenen Ergebnisse wurden mit polarisationsabhängigen Photolumineszenz- [34, 37, 80, 107] oder Kathodolumineszenz-Messungen [81] erzielt. 
Zusätzlich zu der Energiedifferenz zwischen $\Gamma^{1}$ und $\Gamma^{5}$ existiert noch eine $L-T$ Aufspaltung des $\Gamma^{5}$-Zustandes in den transversalen $\Gamma^{5 T}$ und den longitudinalen $\Gamma^{5 L}$-Zustand. Die wenigen existierenden Publikationen hierzu berichten von einer Aufspaltung mit Werten von mindestens $\Delta_{L-T}=5.0 \mathrm{meV}[34$ bis zu einer Größe von $\Delta_{L-T}=7.3 \mathrm{meV} 69$.

Untersuchungen des ternären Verbindungshalbleiters $\mathrm{Al}_{x} \mathrm{Ga}_{1-x} \mathrm{~N}$ berichten von einer Änderung der Polarisation der optischen Übergänge nahe der Bandkante von nahezu $E \perp c(x=0)$ zu fast vollständig $E \| c(x=1)$, wobei eine Entartung aller drei Valenzbänder in Konzentrationsbereichen zwischen $x=0.04$ und $x=0.25$ beobachtet wurde [54, 104, 109].

\section{Abhängigkeit der Valenzbänder von mechanischen Belastungen}

Neben verschiedenen Legierungen hat auch die mechanische Spannung in AlN einen starken Einfluss auf die Position der Valenzbänder. Ikeda et al. haben dafür Untersuchungen des Verhaltens der Valenzbänder für verschiedene dünne Filme auf unterschiedlichen Substraten, hergestellt über MOVPE oder MBE, durchgeführt [54]. Dabei ergab sich aus Berechnungen eine Änderung der Anordnung der Valenzbänder, wenn die mechanische Dehnung $\varepsilon_{z z}$ (in pseudo-kubischer Näherung) einen Wert von $0.70 \%$ überschreitet und ein Anti-Crossing der $\Gamma_{7 \nu}^{+}$und $\Gamma_{7 \nu}^{-}$-Zustände bei einer weiteren Erhöhung auf etwa $\varepsilon_{z z} \approx 0.74 \%$. Zusätzlich zu den beobachteten Verschiebungen fanden Pantha et al. einen linearen Anstieg der Halbwertsbreite bandkantennaher Linien mit steigender Schraubenversetzungs-Dichte, die aus einer Kompensation der anliegenden mechanischen Spannung resultiert [110]. In Abhängigkeit vom verwendetem Substrat beeinflusst eine Dehnung durch nicht genau aufeinander abgestimmte Gitterkonstanten zwischen diesem und einer Dünnschicht-Probe zusammenhängend mit den obigen Studien auch die Größe der Bandlücke. Diese hat dabei einen unmittelbaren Einfluss auf die Energie der rekombinierenden Exzitonen. In Tabelle 2.3 ist eine Auflistung der Rekombinationsenergien vom freien Exziton $F X_{A}$ für verschiedene übliche Substrate gegeben. Dabei führen $\mathrm{SiC}$ und Si normalerweise zu einer biaxialen tensilen Dehnung, was zu einer Reduzierung der Bandlücke führt, wohingegen c-Saphir oder auch GaN/c-Saphir eine kompressive Dehnung mit höheren Rekombinationsenergien verursacht [60]. Eine Möglichkeit zur Reduzierung der Dehnung und damit auch der mechanischen Spannung im AlN bietet eine Segmentierung des unter der AlN-Dünnschicht befindlichen Substrats. Reich et al. fabrizierten hier beispielsweise $10 \mu \mathrm{m}$ dicke Filme auf segmentiertem Saphir über MOVPE [55]. Dabei erreichten sie eine Reduzierung der Schraubenversetzungs-Dichte von $2 \cdot 10^{10} \mathrm{~cm}^{-2}$ auf $9 \cdot 10^{8} \mathrm{~cm}^{-2}$ verglichen zum konventionellem Saphirsubstrat. Ein anderes in den letzten Jahren mehr und mehr Anwendung findendes Substrat ist durch PVT hergestelltes Bulk-AlN, welches an sich a priori schon die idealen Gitterkonstanten aufweist, jedoch aufgrund der Herstellungstechnik nicht in den höchsten Reinheitsgraden dargestellt werden kann. Beim aufwachsen dünner Filme 


\begin{tabular}{|c|c|c|c|c|c|c|}
\hline Sub & $\begin{array}{c}\varnothing \\
(\mathrm{eV})\end{array}$ & $\begin{array}{c}\sigma \\
(\mathrm{meV})\end{array}$ & $\begin{array}{c}\sigma_{r} \\
(\%)\end{array}$ & $\begin{array}{l}\text { Min. } \\
(\mathrm{eV})\end{array}$ & $\begin{array}{l}\text { Max. } \\
(\mathrm{eV})\end{array}$ & Referenz \\
\hline c-Saphir & 6.063 & 30 & 0.49 & 6.031 & 6.138 & \begin{tabular}{|l|l|l|l|l|l|}
21, & 38, & 39, & 41 & 43 & 50 \\
51, & 55, & 58, & 60, & 62, & 63 \\
65 & 68, & 71, & 74 & 78 &
\end{tabular} \\
\hline r-Saphir & 6.033 & 3 & 0.04 & 6.030 & 6.035 & $\mid 69,70,77$ \\
\hline $\mathrm{SiC}$ & 6.027 & 56 & 0.92 & 5.974 & 6.109 & $38,48,49,58,60$ \\
\hline Bulk & 6.0404 & 23 & 0.37 & 6.025 & 6.090 & 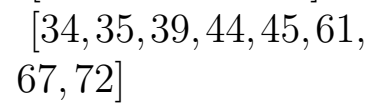 \\
\hline Film auf Bulk & 6.034 & 8 & 0.13 & 6.022 & 6.043 & 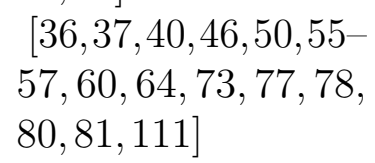 \\
\hline $\mathrm{S}$ & 5.959 & 2 & 0.02 & 5.958 & 5.960 & $|38,60|$ \\
\hline $\mathrm{GaN} / \mathrm{c}-\mathrm{S}$ & 6.045 & 0 & 0.00 & 6.045 & 6.045 & 52,53 \\
\hline
\end{tabular}

Tabelle 2.3: Gemittelte Übergangsenergien von freien Exzitonen $F X_{A}$ in AlN auf verschiedenen Substraten oder in Bulk-Material. Im Fall von Untersuchungen mit Berücksichtigung der Feinstruktur wurde die Energie des $\Gamma^{1}$-Zustandes in die Auswertung mit einbezogen. Die Spalten repräsentieren jeweils den Durchschnittswert $\varnothing$, die Standardabweichung $\sigma$ zwischen den verschiedenen Berichten, die relative Standardabweichung $\sigma_{r}$, die kleinsten und größten gefundenen Übergangsenergien und die zugehörigen Referenzen.

auf solche Bulk-Substrate mittels HVPE oder MOCVD entstehen nahezu spannungsfreie defektarme Filme, welche Versetzungsdichten geringer als $10^{-4} \mathrm{~cm}^{-2}$ aufweisen können [111].

\section{Höher angeregte Exzitonen}

In Proben hoher kristalliner Qualität lässt sich in Lumineszenzexperimenten neben dem Grundzustand des freien Exzitons $F X_{A}$ auch der erste angeregte Zustand $F X_{A}^{2}$ erzeugen. Der zugehörige Rekombinationspeak, welcher in seiner Intensität deutlich abgeschwächt verglichen zu $F X_{A}$ auftritt, wird durchschnittlich 40(5) meV (siehe dazu Tabelle 2.4 ID 4) oberhalb diesem beobachtet. Unter der Annahme eines Verhaltens nach dem Wasserstoffmodell (nach [112]):

$$
E_{B}=\left(\frac{1}{n_{1}^{2}}-\frac{1}{n_{2}^{2}}\right)^{-1} \Delta E
$$

ergibt sich für das freie Exziton $F X_{A}$ eine Bindungsenergie von 53.7(58) meV (mit $n_{1}=1, n_{2}=2$ und $\left.\Delta E=40.4(43) \mathrm{meV}\right)$. Aufgrund dieser hohen Bindungsenergie ist 
das Exziton $F X_{A}$ auch noch bei Raumtemperatur stabil und in Experimenten beobachtbar. Aus einer Addition der durchschnittlichen Exzitonenenergie für ungedehnte Filme von 6.034(8) eV (siehe Tabelle 2.3) und der zuvor berechneten Bindungsenergie ergibt sich für ungedehntes AlN bei tiefen Temperaturen eine Bandlücke von 6.088(10) eV. Aus theoretischer Sicht ist es nicht wirklich korrekt, ein Wasserstoffmodel auf ein Spin-Singulett-Exziton anzuwenden [108]. Jedoch wirken beim $\Gamma^{1}$-Anteil des Exziton $F X_{A}$ die konkurrierenden Effekte des Elektron-Loch-Austauschs und der Exziton-Phonon-Wechselwirkung in Koinzidenz derart gegeneinander, dass eine Anwendung des Modells gute Ergebnisse liefert; im Gegensatz zum $\Gamma^{5}$-Anteil bei dem Ishii et al., unter Annahme einer positiven Wechselwirkungskonstante $j$ beim Elektron-Loch-Austausch, eine Bindungsenergie von $67.3 \mathrm{meV}$ berechneten [108].

\subsubsection{Gebundene Exzitonen}

Neben freien Exzitonen ist auch eine Bindung von Exzitonen an Donatoren oder Akzeptoren möglich, was zu einer Erniedrigung der Rekombinationsenergie führt.

\section{Donatorgebundene Exzitonen}

Bei niedrigen Temperaturen sind die stärksten Lumineszenzen nahe der Bandkante in AlN in der Regel auf Exzitonen, welche an neutrale Donatoren gebunden sind, zurückzuführen (siehe Tabelle 2.4 ID 5). Störstellen im Kristall oder Dotierungen mit Fremdatomen, egal ob absichtlich oder unbeabsichtigt eingebracht, können zu einer großen Zahl von schon bis zu sieben simultan beobachteten Peaks auf der niederenergetischen Seite von $F X_{A}$ führen, welche donatorgebundenen Exzitonen zugeordnet wurden [36]. Ebenso wie das freie Exziton $F X_{A}$ sind sie in ihrer energetischen Position stabil [34] und verhalten sich bezogen zur einfallenden Anregungsleistung in ihrer Intensität nahezu linear [45,70]. Abhängig von der jeweiligen Bindungsenergie zeigen diese Peaks eine starke Verringerung in ihrer Intensität mit steigender Temperatur, da von ihnen ein Transfer in die freien Exzitonenzustände stattfinden kann. Dies begründet auch den bei temperaturabhängigen Messungen zunächst beobachtbaren Anstieg der Rekombinationen der freien Exzitonen mit steigender Temperatur. In Proben hoher kristalliner Qualität kann die Halbwertsbreite von donatorgebundenen Exzitonen Werte geringer als $500 \mu \mathrm{eV}$ [57,111] annehmen. Die energetischen Positionen reichen dabei von $37 \mathrm{meV}$ bis zu $5 \mathrm{meV}$ unter der des jeweilig beobachteten freien Exziton $F X_{A}$. Jedoch ist nur eine kleine Zahl der Peaks spezifischen Donatoren zugeordnet.

Dies trifft für Silizium zu, welches in AlN n-Typ Leitfähigkeit ermöglicht [118, 119]. Das dazu zugehörige Exziton, welches mit Experimenten auf Substraten mit verschiedenen Siliziumkonzentrationen identifiziert wurde (siehe zum Beispiel [56]), befindet sich 26.5(27) meV unterhalb der Position von $F X_{A}$ (siehe Tabelle 2.4 ID 7 ). Dotierungen mit Konzentrationen von bis $\mathrm{zu}(1-3) \cdot 10^{19} \mathrm{~cm}^{-3}$ resultieren in einer 


\begin{tabular}{|c|c|c|c|c|c|c|c|}
\hline ID & $\begin{array}{c}\varnothing \\
(\mathrm{meV})\end{array}$ & $\begin{array}{c}\sigma \\
(\mathrm{meV})\end{array}$ & $\begin{array}{c}\sigma_{r} \\
(\%)\end{array}$ & $\begin{array}{l}\text { Min. } \\
(\mathrm{meV})\end{array}$ & $\begin{array}{l}\text { Max. } \\
(\mathrm{meV})\end{array}$ & Übergang & Referenz \\
\hline 1 & 222.3 & 14.5 & 6.50 & 201.0 & 239.0 & $F X_{C}$ & \begin{tabular}{|l|l|l|}
35, & 38 & 41 \\
\end{tabular} \\
\hline 2 & 208.0 & 13.3 & 6.39 & 187.0 & 223.0 & $F X_{B}$ & \begin{tabular}{|l|l|l|}
35 & 38 & 42 \\
\end{tabular} \\
\hline 3 & 180.7 & 41.8 & 23.1 & 131.7 & 199.0 & $F X_{B C}$ & \begin{tabular}{|l|l|l|}
49 & 53,81 \\
\end{tabular} \\
\hline 4 & 40.4 & 4.3 & 10.7 & 36.0 & 51.7 & $F X_{A}^{2}$ & $\begin{array}{r}\mid 34,36,50,42 \\
51,53,55,58,64 \\
70,80,81\end{array}$ \\
\hline 5 & -18.0 & 6.8 & 37.5 & -37.0 & -5.0 & $D^{0} X$ & \begin{tabular}{|l|l|l|l|l|}
34 & 36, & 42 & 46 \\
50 & 53 & 55 & 58 & 60 \\
64, & 69 & 73 & 77 & 81 \\
110 & 113 & 117 \\
\end{tabular} \\
\hline 6 & -22.6 & 3.8 & 16.9 & -27.0 & -19.3 & $X X$ & {$[34,58,69,70$.} \\
\hline 7 & -26.6 & 2.7 & 10.1 & -28.6 & -22.0 & $S i^{0} X$ & 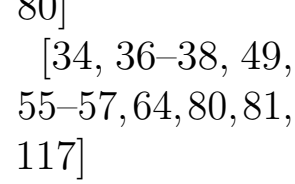 \\
\hline 8 & -26.9 & 1.5 & 5.59 & -28.6 & -26.0 & $O^{0} X$ & [36, 37, 37 \\
\hline 9 & -33.0 & & & & & $B e^{0} X$ & 71 \\
\hline 10 & -35.1 & 0 & 0 & -35.1 & -35.1 & $A^{0} X$ & 69,70 \\
\hline 11 & -37.6 & 3.1 & 8.33 & -41.7 & -34.2 & P-band & $34,44,58,80$ \\
\hline 12 & -40.0 & 0 & 0 & -40.0 & -40.0 & $M g^{0} X$ & 22,68 \\
\hline 13 & -50.0 & & & & & $Z n^{0} X$ & 21 \\
\hline 14 & -76.6 & & & & & $S i^{0} X: 2 s, p$ & 64 \\
\hline 15 & -204.3 & & & & & $E H P$ & 34 \\
\hline
\end{tabular}

Tabelle 2.4: Auflistung der verschiedenen optischen Übergänge nahe der Bandkante, welche in wurtzitischem AlN bestimmten Ursprüngen zugeordnet wurden. Dabei ist die eigentliche Energie relativ zur jeweiligen Position des freien Exzitons $F X_{A}$ gegeben. Im Fall von Untersuchungen mit Berücksichtigung der Feinstruktur wurde die Energie des $\Gamma^{1}$-Zustandes in die Auswertung mit einbezogen. Die Spalten repräsentieren jeweils den Durchschnittswert $\varnothing$, die Standardabweichung $\sigma$ zwischen den verschiedenen Berichten, die relative Standardabweichung $\sigma_{r}$, die kleinsten und größten gefundenen Energien, den zugeordneten Übergang und die zugehörigen Referenzen. 
tensilen Dehnung und einer damit einhergehenden allmählichen Rotverschiebung der beobachtbaren Lumineszenz [120]. Höhere Konzentrationen hingegen führen dann wieder zu einer Blauverschiebung, was mit einer Relaxation des Materials durch die Bildung von Rissen erklärt werden kann [120]. In manchen Fällen wird eine Renormalisierung der Bandkante als Begründung für solche energetische Verschiebungen angegeben [121]. Dies ist für die typischerweise betrachteten Fälle eine jedoch eher unwahrscheinliche Ursache, da die Konzentrationen deutlich niedriger als die kritische von etwa $1.3 \cdot 10^{21} \mathrm{~cm}^{-3}$ [118 liegen. Mit steigender Siliziumkonzentration zeigt sich zudem eine starke asymmetrische Verbreiterung des Lumineszenzsignals hin zu niedrigeren Energien [56]. Das gleiche Verhalten kann auch bei einem Vergleich von gedehnten zu ungedehnten Regionen innerhalb eines Substrates beobachtet werden [122. In der Literatur gibt es verschiedene Ansichten, ob sich Silizium in AlN als substitutioneller Donator auf dem Aluminiumplatz verhält [123 127], oder ob es ein sogenanntes $D X$-Zentrum bildet 128 134]:

$$
2 d^{0} \rightleftharpoons d^{+}+D X^{-}+U \text {. }
$$

Hierbei stehen $d^{0}$ und $d^{+}$für einen substentionellen flachen Donator in neutralem beziehungsweise positivem Ladungszustand, wohingegen $D X^{-}$einem negativ geladenen tiefer liegenden Zustand entspricht. Dieser tritt dabei in einer zur substitutionellen Position verschobenen Lage auf. Wenn die Korrelationsenergie $U$ positiv ist, wird die $D X$-Konfiguration, da energetisch günstiger verglichen zu den zwei neutralen substitutionellen Positionen, bevorzugt [131]. In einer $D X$-Konfiguration wirkt der Donator Silizium selbstkompensierend und kann daher nicht zu einer elektrischen Leitfähigkeit beitragen. Zeisel et al. [131] und Son et al. [133, 134] fanden mit Elektronenspinresonanz-Messungen $D X$-Zustände in einer Tiefe von $320 \mathrm{meV}$ beziehungsweise $78 \mathrm{meV}$ unter dem $d^{0}$-Zustand, während Hevia et al. in Simulationen basierend auf HSE nur Hinweise für kleine Gitterverzerrungen aber keinen Übergang in ein $D X$-Zentrum lieferten [127]. Unabhängig davon beobachteten Neuschl et al. eine frühe thermische Löschung bei etwa $60 \mathrm{~K}$ für den $S i^{0} X$-Übergang, was einer Barriere von $5 \mathrm{meV}$ zwischen einem metastabilen $d^{0}$-Zustand und der $D X$-Konfiguration entsprechen würde [64]. Sie erklären damit einen begleitenden Anstieg der Intensität des freien Exzitons mit dem Vorschlag, dass bei höheren Temperaturen Silizium die Barriere überschreitet und in ein $D X$-Zentrum übergehen kann. Dieses bildet für Exzitonen keinen energetisch attraktiven Zustand, weshalb es nicht zum optischen Spektrum nahe der Bandkante beiträgt. Photoleitfähigkeitsmessungen bei tiefen Temperaturen zeigen einen Schwellwert von etwa $1.5 \mathrm{eV}$ Photonenenergie mit verschwindender persistenter elektrischer Leitfähigkeit und Elektronenspinresonanz-Signalen bei Temperaturen größer als $60 \mathrm{~K}$ [131]. Ungeachtet dessen kann eine relativ "flache" $D X$-Konfiguration (nur $78 \mathrm{meV}$ tiefer als der flache Zustand $d^{0}$ nach [133]) durch thermische Aktivierung bei Raumtemperatur zu einer elektrischen Leitfähigkeit vom n-Typ führen. 
Neben dem am Silizium gebundenen Exziton $S i^{0} X$ wurde auch noch ein ZweiElektronen-Satellit $S i^{0} X: 2 s, p 76.6 \mathrm{meV}$ unterhalb von $F X_{A}$ gefunden [64]. Dieser zeichnete sich bei den Autoren durch ein festes Intensitätsverhältnis von 1:230 verglichen mit $S i^{0} X$ an verschiedenen Probenpositionen und ein gleichartiges temperaturabhängiges Verhalten aus.

Ein weiteres in einer kleinen Zahl von Publikationen identifiziertes Signal ist das an ein Sauerstoffatom gebundene Exziton $O^{0} X$ [36, 37, 57]. Durchschnittlich wurde für die dazugehörige Rekombination eine Übergangsenergie von 26.9(15) meV unter der des freien Exziton $F X_{A}$ bestimmt (siehe Tabelle 2.4 ID 8). Bryan et al. fanden zur Begründung einen nahezu linearen Zusammenhang zwischen der relativen Intensität dieses Peaks und der Sauerstoffkonzentration in ihren MOCVDgewachsenen dünnen Filmen, welche auf PVT Bulk-AlN aufgebracht wurden [36]. Dabei konnten Silizium sowie Kohlenstoff als alternative Ursachen ausgeschlossen werden. Theoretische Untersuchungen ergaben, dass Sauerstoff ein wie weiter oben schon vorgestelltes $D X$-Zentrum mit betragsmäßig hohen Korrelationsenergien von bis zu $U=-2.5 \mathrm{eV}[128$ bildet, im Gegensatz zum Verhalten im nahestehenden GaN, wo es die Position eines flachen Donators einnimmt und somit zu einer elektrischen Leitfähigkeit vom n-Typ führt 100, 124, 125, 127, 129, 132, 135, 137]. In der Legierung $\mathrm{Al}_{x} \mathrm{Ga}_{1-x} \mathrm{~N}$ wurde dabei ein Ubergang vom flachen Donator zum $D X$-Zentrum bei einem zunehmendem Aluminiumanteil im Bereich zwischen $x=0.2[128]$ und $x=0.4[124,125]$ berechnet. Dies kann die starke Reduktion der freien Elektronenkonzentration in unbeabsichtigt dotierten $\mathrm{Al}_{x} \mathrm{Ga}_{1-x}$ N-Legierungen mit steigendem Aluminiumanteil erklären [138,139]. Ebenso wie in siliziumdotiertem AlN deuten EPR-Messungen auch im Fall von Sauerstoff auf eine metastabile nicht-DX-Position hin [140]. Nach einer optischen Anregung mit Photonen einer Energie größer als $\sim 1.8 \mathrm{eV}$ ist ein persistentes EPR-Signal bis zu Temperaturen von $30 \mathrm{~K}$ zu beobachten, was die Annahme eines stabilen tiefen Zustandes für Sauerstoff in AlN unterstützt. Andere Ursprünge für spezifische donatorgebundene Exzitonenübergänge außer im Fall von $S i^{0} X$ und $O^{0} X$ konnten bis jetzt noch nicht identifiziert werden.

Vor kurzem beobachteten Feneberg et al. eine starke Abschwächung der Intensität der Rekombination zweier bis dato nicht identifizierter donatorgebundener Exzitonen und des freien Exzitons $F X_{A}\left(\Gamma^{5}\right)$ nach einem Ausheizvorgang bei $900^{\circ} \mathrm{C}$ unter einem $\mathrm{N}_{2}$ Gasfluss [117]. Nach einem zweitem Ausheizprozess, diesmal mit der Zugabe von $\mathrm{NH}_{3}$ und $\mathrm{H}_{2}$, konnte die Intensität wieder hergestellt werden. Die Proben, welche mit MOCVD hergestellt wurden, zeigten dabei eine messbare Konzentration von Wasserstoff. Für jenes wird erwartet, dass es interstitielle Plätze in AlN einnehmen kann und über einen Ausheizvorgang je nach umgebender Atmosphäre entfernt oder eingebracht werden könnte. Dabei wäre unter den jeweiligen Bedingungen eine Passivierung vorhandener eingeschlossener Donatoren denkbar. Diese Vermutung wird durch theoretische Untersuchungen unterstützt, welche Wasserstoff als amphotere Verunreinigung mit relativ geringen Bildungsenergien voraussagen [141. 


\section{Akzeptorgebundene Exzitonen}

Für eine Vielzahl von Anwendungen in der Halbleitertechnik, wie die Realisierung von Dioden und Transistoren, wird neben der n-Typ-Leitfähigkeit auch eine vom p-Typ benötigt. Bei der Suche nach einer geeigneten Dotierung für elektrische p-Typ-Leitfähigkeit in AlN sind in der Literatur verschiedene mögliche Kandidaten, die einen flachen Akzeptorzustand bilden könnten, in das Material eingebracht worden, was wiederum bei optischen Messungen zu Rekombinationen von Exzitonen mit Bindung an diese Akzeptoren $\left(A^{0} X\right)$ führte [21, 22, 68 71, 79]. Verglichen zu den donatorgebundenen Exzitonen sind die akzeptorgebundenen normalerweise bei niedrigeren Energien in einem Bereich zwischen $50 \mathrm{meV}$ 21 und $33 \mathrm{meV}$ [71 unter der des freien Exzitons $F X_{A}$ zu beobachten (siehe Tabelle 2.4 ID 9, 10, 12 und 13). In experimentellen Messungen konnten die Übergänge $B e^{0} X, M g^{0} X$ und $Z n^{0} X$ mit Energien von jeweils $33 \mathrm{meV}$ [71], $40 \mathrm{meV}$ [22, 68] und $50 \mathrm{meV}$ [21] unterhalb der von $F X_{A}$ gefunden werden. Dabei wurden auch die Lebensdauern dieser sehr kurzlebigen Übergänge auf Zeiten von 93 ps [71], 130 ps [68 beziehungsweise 123 ps [21] bestimmt. Eine momentan in der Literatur noch nicht gelöste Frage ist die des flachsten Akzeptors in AlN. In Bezug auf Haynes Regel [142] hat die Bindungsenergie eines Exzitons an eine neutrale Störstelle eine Größe von etwa $10 \%$ der Bindungsenergie der Störstelle selbst. Im Fall von Be, $\mathrm{Mg}$ und $\mathrm{Zn}$ würde dies $\mathrm{zu}$ jeweiligen Energien von $0.33 \mathrm{eV}, 0.40 \mathrm{eV}$ und $0.50 \mathrm{eV}$ führen, was bei Raumtemperatur nur einen kleinen Teil der eingebrachten Akzeptoren überhaupt aktiviert, wobei demnach Beryllium noch am aussichtsreichsten erscheint. Mit einem Blick auf die Lebensdauern der beobachteten Rekombinationen fanden Sedhain et al. einen weiteren Hinweis darauf, dass sich Beryllium flacher als Magnesium verhält [71]. Sie nutzten dafür die Beziehung:

$$
\frac{\tau_{\mathrm{Be}}}{\tau_{\mathrm{Mg}}}=\left(\frac{E_{\mathrm{Be}}}{E_{\mathrm{Mg}}}\right)^{3 / 2},
$$

wobei $\tau_{\mathrm{Be}}$ und $\tau_{\mathrm{Mg}}$ für die entsprechenden Lebensdauern und $E_{\mathrm{Be}}$ und $E_{\mathrm{Mg}}$ für die zugehörigen Bindungsenergien von Beryllium und Magnesium stehen. Des weiteren existiert eine Anzahl von theoretischen Berichten [126, 143 145], welche auch Beryllium als flachsten Akzeptor mit Bindungsenergien zwischen $0.223 \mathrm{eV}[143]$ und $0.48 \mathrm{eV}$ [145] sehen. Demgegenüber steht eine Simulation von Szab et al. [146], welche nach einem modernen Schema basierend auf HSE Magnesium als den flachsten Akzeptor der gesamten II. Hauptgruppe mit einer Bindungsenergie von $0.50 \mathrm{eV}$ sieht. Zudem widersprechen die Autoren aktiv den Experimenten von Sedhain et al. [71]. Als weiterer Kandidat wurde auch Zink in mehreren theoretischen Untersuchungen als flacher Akzeptor mit niedrigen Aktivierungsenergien vorausgesagt [143, 147, 148], jedoch zeigen sich mit Zink dotierte Filme auch bei Raumtemperatur bis dato hoch resistiv [21]. Umgekehrt wird für Magnesium in theoretischen Publikationen stets eine Bindungsenergie von etwa $0.5 \mathrm{eV}$ ermittelt 124, 126, 136, 143, 145, 149, was im 
guten Einklang mit experimentellen Berichten steht [22, 150]. Der Anteil der freien Ladungsträgerkonzentration folgt der Beziehung $\exp \left(-E_{a} / k_{\mathrm{B}} T\right)\left(k_{\mathrm{B}}\right.$ entspricht hierbei der Boltzmannkonstante). Bei Raumtemperatur und einer angenommenen Aktivierungsenergie von $E_{a}=0.5 \mathrm{eV}$ liegt jedoch nur ein kleiner Teil $\left(4 \cdot 10^{-9}\right)$ der Magnesiumatome in einem ionisierten Zustand vor, was demzufolge nur in einer schwachen p-Leitfähigkeit resultiert. Auch wenn Beryllium als Kandidat für eine mögliche flache Akzeptorposition mit niedriger Aktivierungsenergie in Frage kommt, weisen einige Simulationen auf ein Problem bezüglich der interstitiellen Position $\mathrm{Be}_{\mathrm{i}}$ hin 144 145]. Diese hat laut durchgeführten Berechnungen in einer p-Typ-Umgebung eine niedrigere Bildungsenergie als die für eine Dotierung nötige $\mathrm{Be}_{\mathrm{Al}}$-Position und könnte daher zu einer vollständigen Selbstkompensation führen. Eine Möglichkeit zur Umgehung solcher Probleme, wäre das Arbeiten in thermischen Ungleichgewichtsbedingungen. Hierfür schlagen $\mathrm{Wu}$ et al. $\mathrm{BeH}$ oder $\mathrm{Be}_{2} \mathrm{O}$ vor, welches über eine Kodotierung oder mittels Ionenimplantation mit eingebracht werden könnte [144]. Neben Beryllium kann auch bei Magnesium eine Selbstkompensation (hier über $\left.\mathrm{Mg}_{\mathrm{N}}\right)$ auftreten, was jedoch in einer stickstoffreichen Umgebung eingegrenzt werden kann [145]. Auch die Komplexe $\mathrm{Mg}_{n} \mathrm{O}(n=2,3,4)$ kommen als Kandidaten für flache Akzeptoren in AlN in Betracht. Diese scheinen eine leicht niedrigere Bindungsenergie als $\mathrm{Mg}_{\mathrm{Al}}$ aufzuweisen [146, 149 (z. B. $0.34 \mathrm{eV}$ für $\mathrm{Mg}_{2} \mathrm{O}$ [146]). Ein weiterer Vorschlag kommt von Janotti et al., die bei einer Suche nach geeigneten Elementen auf der rechten Seite des Periodensystems Fluor als möglichen Kandidaten ausfindig gemacht haben [151]. Nach deren Berechnungen verhält sich die interstitielle Position $\mathrm{F}_{\mathrm{i}}$ als ein flacher Akzeptor, welcher bis zu einer Temperatur von etwa $770 \mathrm{~K}$ stabil ist.

Verglichen zu donatorgebundenen Exzitonen zeigen akzeptorgebundene vergleichsweise starke Phononenreplika. Die Begründung hierfür liegt im Bahnmoment des Lochs, während das Donatorelektron nur ein Spinmoment besitzt [79]. Abhängig von der Kristallqualität können mehrere Replika, welche um ein ganzzahliges Vielfaches der Energie von longitudinalen optischen Phononen $A_{1}(\sim 110 \mathrm{meV})[152]$ verschoben sind im Lumineszenzspektrum beobachtet werden. Sedhain et al. bestimmten für freie Exzitonen die dazugehörigen Huang-Rhys-Faktoren $S[153$ in polarisationsabhängigen Photolumineszenzmessungen zu $S^{\perp}=0.78$ für die $E \perp c$ und $S^{\|}=0.11$ für die $E \| c$-Konfiguration [78]. Sie erklären die großen Differenzen zwischen den beiden Polarisationsrichtungen mit der ebenfalls großen Anisotropie der effektiven Lochmasse.

\subsubsection{XX, P-Band und EHP}

Mit zunehmender Intensität bei der Anregung, unabhängig davon ob Photonen, Elektronen oder aber auch Ionen zum Einsatz kommen, steigt auch die Wahrscheinlichkeit für die Bildung von Bi-Exzitonen $(X X)$ an [154]. Dieser Zustand zeigt in seiner Rekombination, verglichen zu den gebundenen Exzitonen, eine relativ breite Linie 
mit einer nichtlinearen Intensitätsabhängigkeit bezogen zur einfallenden Leistung. In AlN wurde eine durchschnittliche Bindungsenergie von 22.6(38) meV $34,58,69,70,80$ (siehe Tabelle 2.4 ID 6) und eine Proportionalitätsbeziehung von $I_{X X} \propto I_{P}^{1.5}$ gefunden [58,70], wobei hier $I_{P}$ der Intensität der Anregung entspricht. Die Streuung der in der Literatur beobachteten Rekombinationsenergien ist relativ groß. Leute et al. erklären diesen Umstand mit verschiedenen mechanischen Spannungen innerhalb der unterschiedlichen untersuchten Proben, was einen Einfluss auf die effektiven Lochmassen im Kristall und somit auch auf die Bindungsenergie der Bi-Exzitonen haben kann [58].

Wenn die Dichte der Exzitonen auf Werte in einen Bereich von $\left(10^{17}-10^{18}\right) \mathrm{cm}^{-3}$ anwächst, erreichen die durchschnittlichen Abstände zwischen ihnen die Größenordnung ihres Radius [155]. In Regionen solch hoher Dichten kann Exziton-ExzitonStreuung (das sogenannte P-Band) auftreten, wobei dort zwei Exzitonen miteinander wechselwirken. Ein Exziton geht dabei in einen energetisch höheren Zustand über $(n=2,3, \ldots)$ oder wird dabei sogar vollständig ionisiert $(n=\infty)$. Im Gegenzug hierzu wechselt das zweite in einen hauptsächlich "photonartigen" Zustand mit erniedrigter Energie und kann den Kristall als Lumineszenz-Photon verlassen [156]. In der Literatur findet sich nur eine kleine Anzahl von Publikationen, bei denen in experimentellen Untersuchungen einem Lumineszenz-Peak Exziton-Exziton-Streuung zugeordnet wurde $34,44,58,80$ (siehe Tabelle 2.4 ID 11). Ähnlich wie bei BiExzitonen zeigt das P-Band auch eine nichtlineare Intensitätsabhängigkeit bezüglich der Anregung mit beobachteten Werten zwischen $I_{\mathrm{P}_{2}} \propto I_{P}^{1.5}\left[34\right.$ bis $I_{\mathrm{P}_{2}} \propto I_{D^{0} X}^{1.66}[58]$. Mit steigender einfallender Leistungsdichte findet eine Rotverschiebung dieses Lumineszenzsignals statt [34,80], welche aufgrund einer größeren Wahrscheinlichkeit für Streuungen in höher angeregte Zustände $(n=3 \ldots \infty)$ resultiert, was wiederum zu einem Überlapp der verschiedenen Bänder $\mathrm{P}_{2} \ldots \mathrm{P}_{\infty}$ führt 34 .

Neben den P-Bändern wurde bei hohen Anregungsleistungen auch schon ein Elektron-Loch-Plasma (EHP) mit einer Ladungsdichte von schätzungsweise etwa $10^{19} \mathrm{~cm}^{-3}$ erzeugt [34]. Dabei wurde von einer Rekombination $200 \mathrm{meV}$ unterhalb von $F X_{A}$ berichtet und ebenfalls ein stärkerer als linearer Anstieg in Abhängigkeit zur Anregungsleistung beobachtet.

\subsection{Defektlumineszenz}

Neben den optischen Übergängen nahe der Bandkante können tief liegende Defekte oder eingebrachte Fremdatome auch innerhalb dieser für Lumineszenz in AlN verantwortlich sein. Grundsätzlich gibt es dabei zwei verschiedene Klassen von beobachtbaren Übergängen. Eine Art sind Interaktionen von freien Ladungsträgern (Elektronen oder Löcher) mit Defektzuständen oder Fremdatomen tief innerhalb der Bandlücke. Diese Rekombinationen zwischen Bändern und Defekten zeichnen sich durch typischerweise sehr kurze Lebensdauern im Bereich von Nanosekunden 
aus und folgen in ihrer Abregung einem exponentiellen Verlauf [157]. Die zweite Gruppe bilden Donator-Akzeptor-Paare (DAPs), bei denen das Energielevel sowohl des Donators $E_{\mathrm{D}}$ als auch des Akzeptors $E_{\mathrm{A}}$ innerhalb der verbotenen Bandlücke liegen. Die Rekombinationsenergie $E_{\gamma}(r)$ für solch einen Übergang ist dabei durch

$$
E_{\gamma}(r)=E_{g}-\left(E_{\mathrm{D}}+E_{\mathrm{A}}\right)+\frac{e^{2}}{\varepsilon r}
$$

gegeben, wobei hier $e$ für die Elementarladung, $\varepsilon$ für die Permittivität bei niedrigen Frequenzen und $r$ für den Abstand zwischen dem beteiligten Donator und dem Akzeptor stehen [158]. Unter der Annahme einer zufälligen räumlichen Verteilung von Donatoren und Akzeptoren im Kristall führt dies zu einem Emissionspeak, welcher zu höheren Energien hin eine Verbreiterung erfährt; insbesondere im Fall von hohen Anregungsintensitäten. Des weiteren besitzen nahe beieinander liegende DAPs kürzere durchschnittliche Rekombinationszeiten als gleichartige weiter voneinander entfernte, was in zeitabhängigen Messungen die Peak-Position mit fortlaufender Zerfallszeit zu niedrigeren Energien hin verschiebt und mit einer zusätzlichen Verringerung der Signalbreite einhergeht $[158,159 \mid$. Dabei zeigen die Übergänge, im Gegensatz zur vorher beschriebenen ersten Gruppe, ein zeitlich nicht exponentielles Verhalten. Insbesondere der langlebige Bereich kann über Potenzgesetze gut abgebildet werden [157, 158. Bei hohen Störstellenkonzentrationen kann die von den DAPs ausgehende Lumineszenz auch gelöscht werden. Dies führt zu scheinbar kurzlebigeren Zerfallszeiten, welche dann auch als Transienten mit exponentieller Verlaufsform beobachtet werden können [158, 159]. In AlN sind die in Experimenten gefundenen Lumineszenzen mit Energien innerhalb der Bandlücke einer Vielzahl von Ursprüngen, sowohl Übergängen zwischen Bändern und Defektleveln als auch von DAPs, zugeordnet worden. Eine zusammenfassende Liste ist in Tabelle 2.5 gegeben.

Genau wie für bandkantennahe Übergänge existiert auch eine große Anzahl an Simulationen, die sich mit den energetischen Positionen verschiedener Defekte innerhalb der Bandlücke von AlN befassen [96 100, 123 130, 132, 135 137, 141, 143, 147, 148, 160 166]. Einige dieser Berechnungen wurden dabei in Zinkblendenstruktur durchgeführt [128, 129, 137, 141, 147, 148, 161, 166]. Die Autoren in diesen Berichten gehen daraufhin auf ein gleichartiges Verhalten der Defektzustände in wurtzitischem AlN aus. Insbesondere frühere Untersuchungen, welche sich mit den Energieniveaus verschiedener Defekte auseinandersetzen, leiden an einer erheblichen Unterschätzung der Bandlücke von AlN. Dies führt so auch für die berechneten Defektniveaus zu einem zu kleinen energetischen Bereich. In einigen Publikationen [147, 148, 160 wird durch die Einführung eines "Scherenoperators" versucht, ein dem System angepassteres Ergebnis zu generieren. Dabei erfolgt im Nachhinein eine Vergrößerung der Bandlücke durch Skalierung jener als auch einer damit einhergehenden Neupositionierung der berechneten Energielevel.

Abhängig vom Verhältnis zwischen den Stickstoff- und Aluminium-Vorläuferverbindungen während des Wachstums der AlN-Präparate, herrschen auf der Wachs- 
tumsoberfläche unterschiedliche chemische Potentiale vor. Diese führen wiederum zu verschiedenen Energien für die Bildung bestimmter Defekte oder auch Defektkomplexe. Die zwei in der Literatur betrachteten Fälle sind N-reiche oder Al-reiche Bedingungen (für weitere Details sei auf das Review [167] oder Referenz [127] verwiesen). In Experimenten mit MOCVD-gewachsenen Präparaten wird häufig angenommen, dass diese Experimente unter Al-reichen Bedingungen stattfinden, da $\mathrm{N}_{2}$ eine sehr hohe Bindungsenergie besitzt. Theoretische Untersuchungen von Li und Brenner [168] suggerieren eine Untersättigung von $\mathrm{N}_{2}$ bezüglich der Kristalloberfläche, was dieses als Vorprodukt ausschließt. Hingegen diesem können sich indes $\mathrm{Al}_{n} \mathrm{~N}(n=2,3,4)$-Verbindungen als Stickstoff-beinhaltendes Vorprodukt in einer übersättigten Konzentration bilden, was zu einer möglichen N-reichen Wachstumsumgebung führen kann. Somit könnten dann in solchen Wachstumsprozessen andere Bildungsenergien als experimentell vorher erwartet für die jeweiligen Defekte auftreten, welche dadurch in ihren Konzentrationen stark beeinflusst würden.

Eine kompakte Übersicht zu den in der Literatur publizierten theoretischen Simulationen von verschiedensten Defekten oder anderen Inkorporationen in AlN ist in Tabelle A.1 gegeben. In den nun folgenden Abschnitten wird auf die verschiedenen in der Literatur sowohl theoretisch als auch experimentell untersuchten Defekte oder andere Fremdatome in AlN in Bezug zu Lumineszenzerscheinungen genauer eingegangen. Dabei findet eine Gegenüberstellung der unterschiedlichen Interpretationen statt.

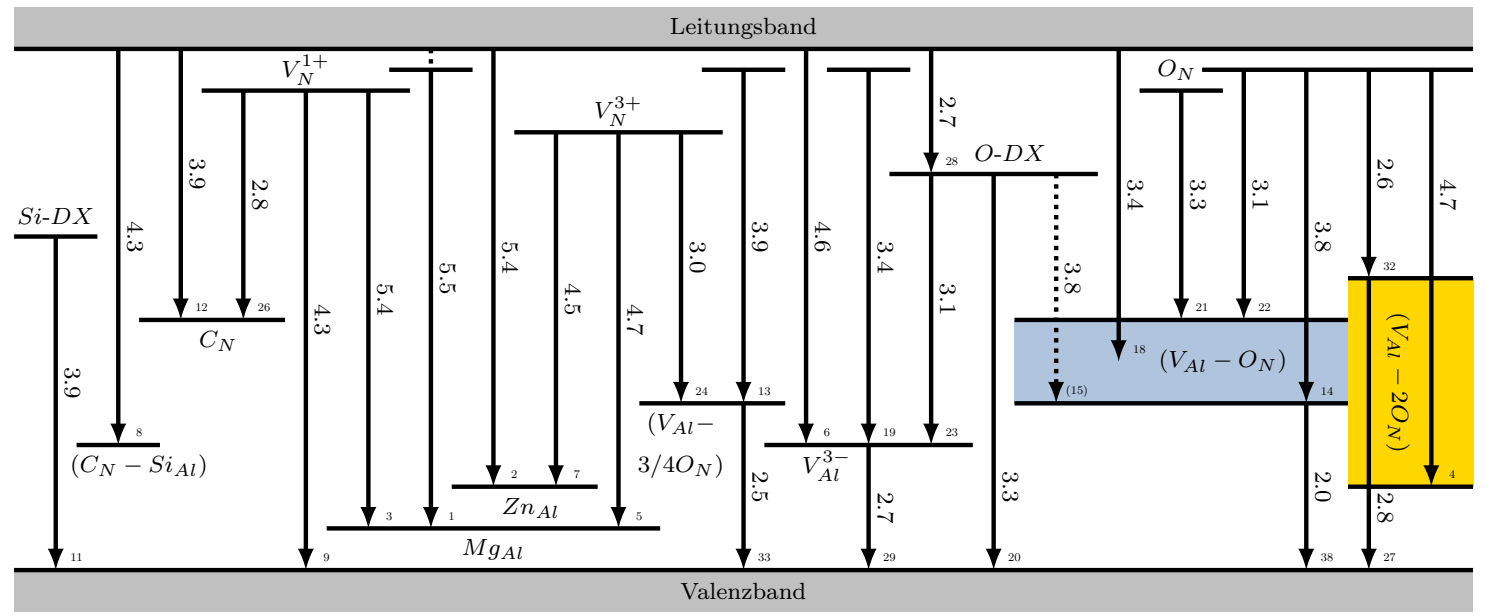

Abbildung 2.2: Graphische Darstellung der Übergänge unterhalb der Bandkantenregion. Die Energien sind in eV gegeben. Die kleinen Nummern an den Pfeilspitzen beziehen sich auf die ID-Spalte in Tabelle 2.5. Die farbigen Gebiete repräsentieren die jeweiligen großen Energiebereiche der Übergänge, bei welchen $\left(V_{A l}-O_{N}\right)$ oder $\left(V_{A l}-2 O_{N}\right)$ mit involviert sind. Der Übergang beschriftet mit ID 15 (gepunktet) ist nicht exklusiv dem $\left(V_{A l}-O_{N}\right)$-Komplex zugeordnet (nach [29]). 


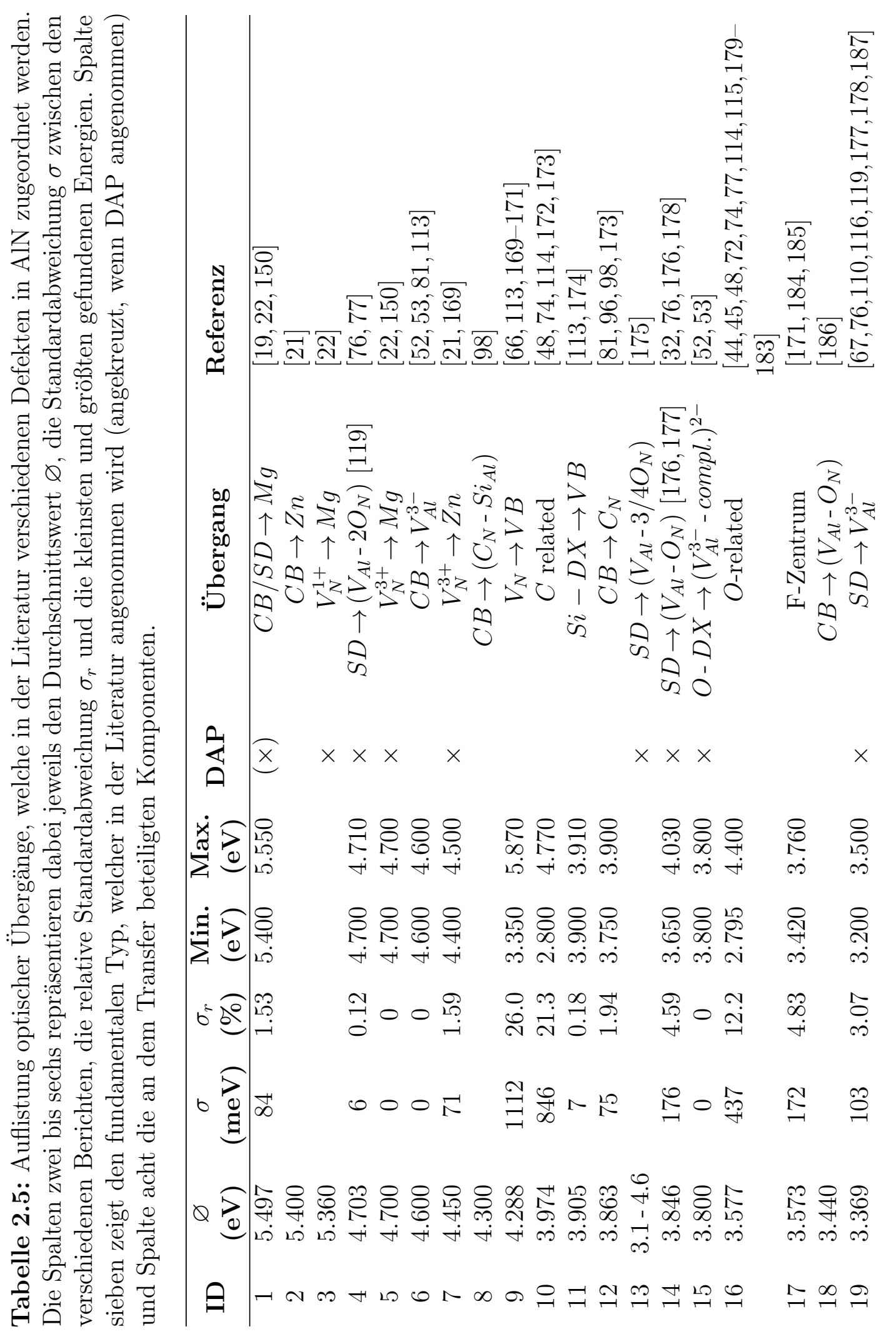




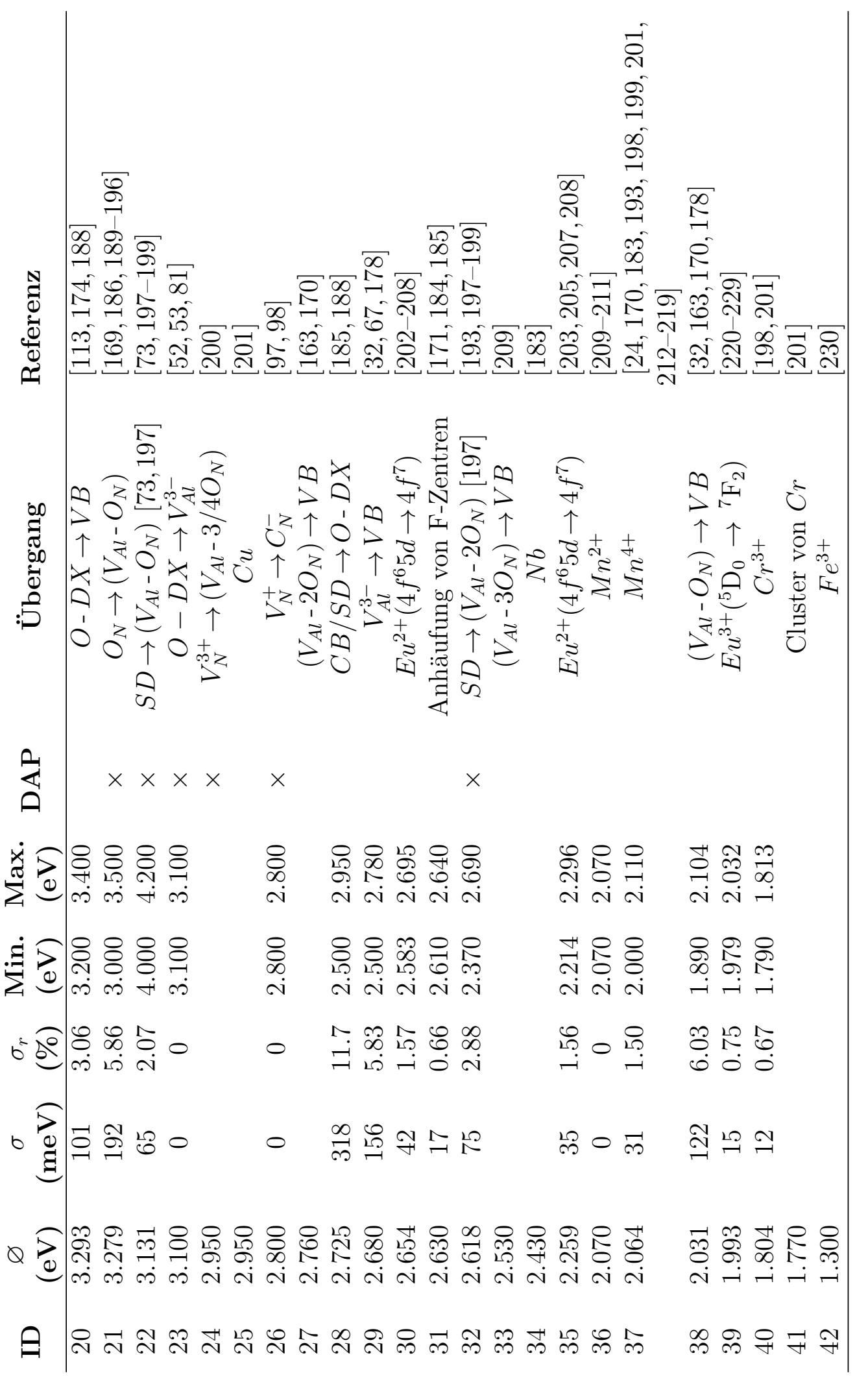




\subsubsection{Intrinsische Defekte}

Bei der Herstellung von AlN können sich je nach chemischer Umgebung und Herstellungsverfahren verschiedene intrinsische Punktdefekte, wie Leerstellen, Vertauschungen von Atomen oder auch die Besetzung interstitieller Atomplätze, ausbilden. Diese können maßgeblich zum Lumineszenzverhalten von AlN mit beitragen. In theoretischen Untersuchungen wurden in der Literatur die Leerstellen des Aluminiums $\left(V_{A l}\right)$ als auch die des Stickstoffs $\left(V_{N}\right)$ am häufigsten untersucht $97,100,123,124,126,127,129,136,137,145,147,148,160$ 162, 164].

\section{Aluminiumleerstelle}

$V_{A l}$ kann den meisten theoretischen Berechnungen folgend in den Ladungszuständen zwischen "0" und "3-" auftreten [97, 100, 126, 129, 145, 147, 160, 161]. Zusätzlich wurde in manchen Berichten auch ein stabiler "+"-Ladungszustand in einer weit im p-Bereich liegenden Umgebung gefunden [97, 100, 126]. Dieser Zustand besitzt jedoch unabhängig von den Wachstumsbedingungen eine hohe Bildungsenergie und sollte deshalb nur in verschwindend geringen Konzentrationen auftauchen. Im Gegensatz zu den anderen Ergebnissen weisen die Simulationen von Hevia et al. nur auf eine Stabilität der Zustände $V_{A l}^{0}$ und $V_{A l}^{3-}$ hin [127]. Abhängig von der jeweiligen Simulation zeigt $V_{A l} \mathrm{im}$ "3-"-Ladungszustand die niedrigste Bildungsenergie startend in einem Fermilevel-Bereich zwischen $0.8 \mathrm{eV}$ [127] und $3 \mathrm{eV}$ [97] bis hin zur Energie der Bandkante. Insbesondere unter stickstoffreichen Bedingungen verringert sich die Bildungsenergie von $V_{A l}^{3-} \mathrm{zu}$ extrem niedrigen Werten, wodurch die Leerstelle als stark kompensierender Akzeptor bei einer n-Dotierung agieren und damit zu einer drastischen Verringerung der erreichbaren n-Leitfähigkeit führen kann. In AlGaNLegierungen nimmt die Bildungsenergie von $V_{A l}$ mit zunehmendem Aluminiumanteil immer weiter ab, was zusätzlich zum Übergang des Sauerstoffs in ein $D X$-Zentrum (siehe Kapitel 2.1.3) eine Erklärung für die damit einhergehende sich immer weiter vermindernde intrinsische n-Leitfähigkeit liefern kann [124. Hung et al. fanden in ihren Untersuchungen große Unterschiede in den energetischen Positionen von $V_{A l}$ in Abhängigkeit von den verwendeten Simulationsmethoden [162]. Sie berechneten in den auf GGA oder B3LYP basierenden Ansätzen sehr unterschiedliche Positionen von jeweils $0.3 \mathrm{eV}$ und $1.4 \mathrm{eV}$ oberhalb des Valenzbandmaximums [162].

In der Literatur wird unter anderem eine experimentell beobachtete Lumineszenz einem Übergang zwischen dem Leitungsband und dem $V_{A l}^{2-/ 3-}$-Zustand zugeordnet, wobei die Veröffentlichungen einheitlich von einer Rekombinationsenergie von $4.6 \mathrm{eV}$ berichten (siehe Tabelle 2.5 ID 6). Unter der Ausnutzung von PositronenAnnihilations-Messungen und verschiedenen III/V-Verhältnissen beim Wachstum der Präparate konnte $V_{A l}$ als ein Teil der beobachteten Lumineszenz bestätigt werden 52,53,113. Mit sinkendem III/V-Verhältnis steigt die Intensität des Signals an, was mit niedrigeren Bildungsenergien und einem damit vermehrten Auftreten der 
Leerstelle aufgrund stickstoffreicherer Bedingungen begründet werden kann [113. Des weiteren korrespondiert auch der S-Parameter aus Annihilationsexperimenten, welcher sensitiv auf die $V_{A l}$-Konzentration ist, mit der Intensität dieser Lumineszenz [52,53]. Bei einer durchschnittlichen Rekombinationsenergie von 3.37(11) eV berichten mehrere Publikationen von einem DAP-Übergang zwischen einem unbekannten flachen Donator und $V_{A l}^{2-/ 3-}$ (siehe Tabelle 2.5 ID 19). Alle diese Berichte außer derjenige von Schulz et al. [187] beziehen sich direkt oder auch indirekt auf eine Publikation von Nam et al. [177]. Die dortige Zuordnung basiert jedoch lediglich auf einen Vermerk zu zwei theoretischen Berechnungen 129, 136. Schulz et al. [187] fanden eine von der Anregungsintensität abhängige energetische Verschiebung, welche typisch für DAP-Übergänge ist. Überdies beschreiben sie eine Umwandlung bei Temperaturen oberhalb von $100 \mathrm{~K}$ hin zu dem schon vorher geschilderten Übergang vom Leitungsband zur Leerstelle. In Absorptionsmessungen wurde korrespondierend mit diesem Übergang ein Band mit 3.59 eV gefunden [231], was einer Stokes-Verschiebung von etwa $0.2 \mathrm{eV}$ entspricht. Verglichen mit dem Transfer vom Leitungsband müsste damit der "flache" Donator eine Tiefe von etwa $1.2 \mathrm{eV}$ besitzen, was die großen Diskrepanzen bezüglich der Interpretation dieser beiden Übergänge in der vorhandenen Literatur verdeutlicht. Bezugnehmend auf den DAPTransfer zwischen einem flachen Donator und dem Zustand $V_{A l}^{2-/ 3-}$ existiert auch eine kleine Anzahl von Berichten, welche eine Rekombination bei durchschnittlich 2.68(16) eV einem Übergang zwischen der Leerstelle $V_{A l}$ und dem Valenzband zuordnet (siehe Tabelle 2.5 ID 29). Die Veröffentlichungen stützen sich hierbei auf das in Referenz [178] angegebene Energielevel für $V_{A l}$, welches wiederum aus der vorher schon dargestellten Publikation von Nam et al. [177] herrührt. Sedhain et al. 32, 67] fanden für den Übergang keinen energetischen Drift des Signals mit unterschiedlichen Anregungsintensitäten und schließen somit einen DAP-Transfer aus. Weiterhin erwarten sie mit steigender Sauerstoffkonzentration in den Proben auch einen Anstieg der $V_{A l}$-Konzentration, welches sich auf theoretische Berechnungen stützt 129, 136. Dieser Schlussfolgerung widersprechen Gaddy et al. [97], da in den betrachteten Proben in m-Ebene, welche über PVT hergestellt wurden, mögliche Kohlenstoffkontaminationen vorhanden sein können. Des weiteren nehmen Sedhain et al. an, dass es sich bei dem betrachteten Grundzustand um den Ladungszustand "2-" handelt. Jener ist allerdings nur in einem sehr kleinen leicht in Richtung p-Typ verschobenem Bereich des Fermilevels vorhanden (sehe z. B. 97, 100, 129]).

\section{Stickstoffleerstelle}

In AlN mit p-Dotierung, insbesondere unter aluminiumreichen Bedingungen, sind die Bildungsenergien für Stickstoffleerstellen $V_{N}$ sehr niedrig (siehe z. B. [97, 100]). Da sich $V_{N}$ typischerweise wie ein Donator verhält, können diese Leerstellen als Kompensationszentren wirken, was neben der hohen Aktivierungsenergie der bis jetzt gefundenen Akzeptoren (siehe Kapitel 2.1.3) einer effektiven p-Dotierung entgegenwirkt. Die 
aus Simulationen typischerweise gefundenen stabilen Ladungszustände sind "+" und " $3+$ ", während " $2+$ " als instabil gilt oder lediglich nur in einem sehr schmalen Bereich des Fermilevels stabil auftauchen kann $97,100,124,127,129,136,137,145,160,164$. Bei Fermileveln bis hin zur Mitte der Bandlücke ist die Bildungsenergie niedriger als jene von $V_{A l}$; selbst in einer stickstoffreichen chemischen Umgebung (siehe z. B. 97, 100, 127]). Des weiteren scheint auch der neutrale Ladungszustand instabil, oder lediglich in einem kleinem Bereich des Fermilevels bevorzugt zu sein. Berechnungen von Ye et al. ergeben eine Stabilität von $V_{N}^{0}$ nur dann, wenn es sich bei den Gittern um nicht relaxierte handelt, während bei relaxierten dieser Ladungszustand instabil erscheint 164. In AlN mit Fermileveln Richtung n-Typ zeigt $V_{N}$ sein amphoteres Verhalten mit Ladungszuständen von bis zu "3-". Diese können im Fall einer aluminiumreichen Umgebung auftreten und besitzen dabei mit $V_{A l}^{3-}$ vergleichbare Bildungsenergien (siehe z. B. [127, 129, 137]). Wenn $V_{N}$ in solch einem Ladungszustand vorliegt, zeigen die adjazenten Atome eine stark von der Leerstelle nach außen verschobene Positionierung [164.

In Lumineszenzmessungen wurde von einigen Gruppen ein Peak bei 4.3(12) eV einem Übergang von $V_{N}$ zum Valenzband zugeordnet (siehe Tabelle 2.5 ID 9). Die dabei gefundenen Werte zeigen eine breite Verteilung mit Rekombinationsenergien von $2.86 \mathrm{eV} 232$ bis zu $5.87 \mathrm{eV}$ [66]. Dieser Bereich entspricht nahezu der kompletten spektralen Region, welche bei typischen Lumineszenzexperimenten in AlN untersucht werden. Ein Problem hierbei ist eine in späterer Literatur oft fehlinterpretierte und daraufhin fehlerhaft zitierte Absorptionsmessung von Cox et al. 233. Ein Absorptionspeak bei $2.8 \mathrm{eV}$ wird in dieser Publikation tendenziell interstitiellem Aluminium $\left(A l_{i}\right)$ zugeordnet, von anderen auf diese Veröffentlichung Bezug nehmend jedoch der Stickstoffleerstelle [179, 232]. Es existieren auch einige Berichte, unter anderem auch der von Cox et al., in denen ein Absorptionspeak bei $5 \mathrm{eV}$ der Stickstoffleerstelle zugeschrieben wird [178, 188, 233, 234]. Somit erscheint auch eine eher höhere Rekombinationsenergie für $V_{N}$ dahingehend wahrscheinlicher. Ungeachtet dessen gibt es auch eine Veröffentlichung von Atobe et al. in der ein Absorptionspeak bei $3.35 \mathrm{eV}$ explizit $V_{N}$ zugeordnet wird [235]. Die Autoren begründen dies mit einer Zunahme der Absorption mit steigender Neutronenbestrahlung und vergleichen ihr Ergebnis mit einem analogen Verhalten in Oxiden. Unter Zuhilfenahme von ESR-Messungen erklären sie einen beobachteten Elektroneneinfang mit der Bildung von $V_{N}^{3+}$. Diesen Ergebnissen folgend ordneten $\mathrm{Hu}$ et al. Lumineszenzsignale aus Bestrahlungsexperimenten mit einer Rekombinationsenergie von $3.35 \mathrm{eV}$ der Stickstoffleerstelle zu 170, 171. Im Widerspruch dazu schließen Yan et al. einen Absorptionspeak von $V_{N}$ um $3.4 \mathrm{eV}$ aus und erwarten dementsprechende Signale oberhalb von $5.1 \mathrm{eV}$ oder unterhalb von $2.3 \mathrm{eV}$ [100]. Basierend auf ihren Simulationen weisen sie den $3.4 \mathrm{eV}$-Peak eher $V_{A l} \mathrm{zu}$.

Komplexe von Leerstellen wie $V_{A l}-V_{N}$ zeigen verglichen zu den vereinzelten $V_{A l}$ und $V_{N}$ höhere Bildungsenergien und sollten daher auch nur in relativ geringen Konzentrationen in AlN auftauchen [129]. 


\section{Anti-site-Defekte}

Neben theoretischen Untersuchungen von Leerstellen in AlN existieren auch einige Simulationen, welche sich mit den substitutionell vertauschten Aluminium auf Stickstoff- $\left(A l_{N}\right)$ und Stickstoff auf Aluminium-Gitterfehlern $\left(N_{A l}\right)$ auseinandersetzen 123, 127, 136, 145, 147, 148]. $A l_{N}$ besitzt demnach relativ niedrige Bildungsenergien in p-Typ AlN, dabei unter aluminiumreichen Wachstumsbedingungen mit einem Ladungszustand von "3+" [145] oder unter einer großen Verschiebung aus der substitutionellen Position um etwa $28 \%$ der Gitterkonstanten mit einem Ladungszustand von "4+" [136]. Jedoch liegt in beiden Fällen die nötige aufzubringende Energie zur Bildung höher als jene von $V_{N}^{3+}$. Zhang et al. ordnen einen Absorptionspeak um $1.5 \mathrm{eV}$ dem $A l_{N}$-Defekt unter der Begründung verschiedener stöchiometrischer Verhältnisse innerhalb ihrer Proben zu [188].

Im Gegensatz dazu besitzt $N_{A l}$ unabhängig von den gegeben Wachstumsbedingungen hohe Bildungsenergien verglichen mit anderen intrinsischen Defekten in AlN [136, 145]. Experimentelle Untersuchungen in der Literatur, bei denen explizit optische Rekombinationen den Gitterfehlern $A l_{N}$ oder auch $N_{A l}$ zugeordnet wurden, sind im Rahmen der hier durchgeführten weitreichenden Literaturrecherche nicht aufgetreten.

\section{Interstitielle intrinsische Defekte}

Interstitielle intrinsische Defekte, wie $A l_{i}$ und $N_{i}$ wurden ebenfalls in theoretischen Publikationen nur selten untersucht 124, 127, 136, 145, 147, 151]. In wurtzitischem AlN zeigen sowohl $A l_{i}$ als auch $N_{i}$ verglichen zu anderen intrinsischen Defekten relativ hohe Bildungsenergien. Nach Stampll et al. besitzt $A l_{i}^{3+}$ lediglich in aluminiumreichen Bedingungen mit einem Fermilevel weit in der p-Region niedrige Bildungsenergien, welche dennoch etwa $2 \mathrm{eV}$ über denen in kubischen AlN liegen [124, 136]. Im Gegensatz dazu fanden Zhang et al. unabhängig von den vorliegenden Umgebungsbedingungen stets hohe Bildungsenergien [145]. Für $N_{i}^{3-}$ berechneten Janotti et al. niedrige Bildungsenergien in n-Typ AlN [151], was im Widerspruch zu den Ergebnissen von Stampfl et al. steht, welche zu allen Bedingungen hohe Bildungsenergien voraussagen [136.

Aufgrund der hohen nötigen Energie zur Erzeugung dieser Defekte und einer damit einhergehenden erwarteten niedrigen Konzentration jener, wurden im Rahmen der durchgeführten Literaturrecherche keine beobachteten Lumineszenzen explizit dem interstitiellem Aluminium- oder Stickstoffdefekt zugeordnet. Cox et al. führen einen Absorptionspeak bei $2.8 \mathrm{eV}$ tendenziell auf $A l_{i}$ zurück, da sich dieser nach dem Einbringen von Aluminium in die Probe verstärkte [233. Hierfür beschichteten sie ihre Proben mit Aluminium und erhitzten sie für zwei Stunden bei $1100^{\circ} \mathrm{C}$. 


\section{Defekterzeugung im Ungleichgewicht}

Obwohl die Energie zur Bildung der meisten intrinsischen Defekte sehr groß unter den verschiedenen Gleichgewichtsbedingungen beim Wachstum von AlN ist, können jene per Neutronen- oder Ionenbestrahlung erzeugt werden [170, 171, 184, 185, 220, 235]. Sie zeigen dabei eine hohe über Raumtemperatur hinaus gehende thermische Stabilität. Insbesondere die im Nachhinein eingebrachten Leerstellen und interstitiellen Defekte können zu zusätzlichen Lumineszenzpeaks in AlN führen. Hu et al. [171,184 beobachteten einen zunehmenden Peak mit steigender Ionenfluenz, welchen sie der Stickstoffleerstelle $V_{N}$ zuordnen. Demnach fängt die Leerstelle dabei mindestens ein Elektron ein und bildet ein Farbzentrum bei einer energetischen Position von etwa 3.58(18) eV (siehe Tabelle 2.5 ID 17). Mit weiter ansteigender Ionenfluenz nimmt in den durchgeführten Experimenten ein zweites Signal bei 2.63(2) eV (siehe Tabelle 2.5 ID 31) zu, welches als eine Aggregation von Farbzentren interpretiert wird. Hu et al. erwarten eine niedrigere energetische Position für die gruppierten Farbzentren, da es sich um einen höheren Schaden im Wirtsgitter handelt und fanden ein erhöhtes Intensitätsverhältnis zwischen aggregierten und einzelnen Farbzentren im Fall von Implantationen bei höheren Temperaturen [184]. Vetter et al. beobachteten eine ausgeprägte Löschung der vorher sichtbaren Lumineszenz der Farbzentren durch eine Koimplantation von Fluor, was möglicherweise zu einer Reduzierung der Anzahl der in den Proben vorhandenen Stickstoffleerstellen geführt haben könnte [185]. Ungeachtet dessen bleibt die Zuordnung zu $V_{N}$ eher fragwürdig, da die Übergangsenergien Yan et al. [100] folgend eher zu $V_{A l}$ passen würden.

\subsubsection{Sauerstoff}

Neben intrinsischen Defekten gibt es eine ganze Reihe von Fremdatomen, die während des Probenpräparationsprozesses in das AlN eingebracht werden, wo sie dann zum Defektlumineszenz-Spektrum einen signifikanten Beitrag leisten können. Meist ungewollt, aber bei der Präparation schwierig zu vermeiden ist die Inkorporation von Sauerstoff. Abhängig von den Wachstumsbedingungen sind dabei Konzentrationen bis zur Löslichkeitsgrenze von etwa $2 \cdot 10^{21} \mathrm{~cm}^{-3}$ bei etwa $2000{ }^{\circ} \mathrm{C}(\sim 2$ at. \%) oder $6 \cdot 10^{21} \mathrm{~cm}^{-3}$ bei $100^{\circ} \mathrm{C}(\sim 6$ at. \%) möglich [6]. In manchen Veröffentlichungen berichten die Autoren auch von Sauerstoffkonzentrationen, welche sich oberhalb dieser Grenzen befinden [188, 212, 236 239]. Vermutlich bildet sich in Proben mit solch hohen Sauerstoffanteilen eine Phasenseparation von Aluminiumoxid aus [6, 212]. Wie schon zuvor in Kapitel 2.1.3 dargestellt, herrscht in der Literatur relative Einigkeit darüber, dass Sauerstoff in AlN als Grundzustand ein DX-Zentrum bildet. Ein Lumineszenzband mit einer durchschnittlichen Energie von 3.29(11) eV (siehe Tabelle 2.5 ID 20) wird einer Rekombination dieses Defektzustandes mit dem Valenzband zugeschrieben. Jedoch basiert jene Zuordnung lediglich auf frühen theoretischen Untersuchungen, ohne dabei alternative weiter unten aufgeführte 
komplexere Defektstrukturen mit in Betracht zu ziehen [113, 174]. Bickermann et al. untersuchten PVT-gewachsene Proben, welche eine Sauerstoffkonzentration von nur etwa 100 ppm wt. aufwiesen und ordneten daraufhin einen Absorptionspeak bei $5 \mathrm{eV}$ Sauerstoff zu [178, 231, was nicht zu einer Rekombinationsenergie um 3 eV korrespondieren kann. Ungeachtet dessen wurde ein Lumineszenzpeak bei 2.7(3) eV (siehe Tabelle 2.5 ID 28) einem Übergang zwischen einem flachen Donator beziehungsweise auch direkt vom Leitungsband und dem Sauerstoffdefekt zugeordnet, jedoch ohne irgendwelche darauf hindeutende Argumente zu liefern [188]. Verglichen zu anderen Ergebnissen führt dies zu einer Annahme eines extrem tiefen $D X$-Zustandes [140]. Die Gruppe um Chichibu weist einen Lumineszenzpeak bei $3.1 \mathrm{eV}$ (siehe Tabelle 2.5 ID 23) einem DAP-Übergang zwischen Sauerstoff und der Aluminiumleerstelle zu. Sie stützen dabei ihre Ergebnisse mit Positronen-Annihilationsexperimenten, welche sensitiv auf die Leerstelle $V_{A l}$ reagieren [52,53]. Des weiteren motivieren sie ihre Zuordnung mit Hilfe eines früheren Berichts von Nam et al. [177], die die energetische Position von $V_{A l}^{3-}$ nahe der Mitte der Bandlücke, etwa $0.5 \mathrm{eV}$ über einem Defekt der Form $\left(V_{A l}-\text { complex }\right)^{2-}$ ansiedeln. Verglichen zu ihrer simultanen Zuordnung des in Kapitel 2.2.1 vorgestellten Übergangs zur Aluminiumleerstelle (siehe Tabelle 2.5 ID 6) bleibt die Argumentationskette in diesem Fall nicht vollständig widerspruchsfrei. Wie anhand der hier vorgestellten Interpretationen und Zuordnungen von Lumineszenzen in Verbindung mit dem Sauerstoffdefekt erkennbar, gestaltet sich eine eindeutige Identifikation als schwierig. Noch weitaus komplizierter sieht es mit der Zuordnung von Lumineszenzen in Verbindung mit Leerstellen-Sauerstoff-Komplexen aus, auf welche im nächsten Abschnitt näher eingegangen wird.

Die in der Literatur mit Sauerstoff in Verbindung gebrachten Lumineszenzen zeigen eine extrem breite Verteilung über einen weiten Bereich von $2.7 \mathrm{eV}$ [183] bis hin zu $4.7 \mathrm{eV} \mid 76$ mit verschiedensten zum Teil im Widerspruch stehenden Zuordnungen. Eine frühe Theorie für $\left(V_{A l}-O_{N}\right)$ und $\left(V_{A l}-2 O_{N}\right)$-Komplexe mit den dazugehörigen Energieniveaus wurde von Pastrňák et al. in der Mitte der 70er Jahre entwickelt [197]. Sie fitteten ihre Anregungs- und Emissionsspektren mit sechs respektive fünf Normalverteilungen an. Die dabei gewählten jeweiligen Zentralwerte als auch Halbwertsbreiten wurden für alle experimentellen Bedingungen miteinander verknüpft und somit gleich gesetzt. Von diesen fünf Lumineszenzpeaks ordneten sie die Signale bei $2.96 \mathrm{eV}$ und $3.23 \mathrm{eV}$ einem DAP-Übergang zwischen einem unbekanntem flachen Donator und dem $\left(V_{A l}-O_{N}\right)$-Komplex zu, wobei jener in zwei verschiedenen Konfigurationen vorliegen soll. Die erste Rekombination ist dem $\left(V_{A l}-O_{N}\right)$-Komplex zugeordnet, wo $O_{N}$ sich in der zweiten Anionen-KoordinationsSphäre ( $\pi$-Konfiguration) befindet. Bei der zweiten, welche eine höhere Energie besitzt, befindet sich $O_{N}$ in der Position des nächsten Nachbarn ( $\sigma$-Konfiguration). Analog dazu schreiben sie zwei Lumineszenzen, die jeweils etwa $0.5 \mathrm{eV}$ unterhalb der vorher beschriebenen Defekte auftauchen, dem Komplex $\left(V_{A l}-2 O_{N}\right)$ zu. Hierbei hat die Konfiguration nach dem $\pi$-Typ ein $O_{N}$ als nächsten Nachbarn, während sich das andere in der zweiten Anionen-Koordinations-Sphäre befindet. 
Der Idee der DAP-Rekombination von Pastrňák et al. [197] folgend, entwickelten Harris und Youngman ein geladenes Störstellenmodell [240|. Dabei gehen sie von einer Kompensation von drei Sauerstoffatomen durch eine Aluminiumleerstelle aus. In Analogie zu einer Theorie in GaAs [241] erklären sie das in Richtung der Bandkante ihrer Proben auftauchende stetig ansteigende Absorptionsband mit einer hohen Konzentration von geladenen weit verteilten Defekten. Zusätzlich, unter Anwendung ihres Modells, können sie die schwache Temperaturabhängigkeit der energetischen Positionen und die breiten Signalformen, welche auch in Experimenten bei tiefen Temperaturen auftauchen, mit örtlichen Bandlückenfluktuationen erklären. Nach ihrer Theorie kann eine beobachtete Rotverschiebung in zeitabhängigen Messungen durch Rekombinationen von weiter entfernt voneinander liegenden Defekten erklärt werden (siehe auch Kapitel 2.2). Darauf basierend entwickelten sie ein Modell, welches einige der verschiedenen Eigenschaften von mit Sauerstoff in Verbindung gebrachten Lumineszenzen erklären kann 176, 242. Wenn die Sauerstoffkonzentration ihrer Proben einen Wert von 0.75 at. \% überschritt, beobachteten sie einen abrupten Anstieg der Rekombinationslebensdauer und eine Verringerung des AlNZellenvolumens, sowie der thermischen Leitfähigkeit. Sie begründen dieses Verhalten mit dem Auftauchen eines neuen Defekttyps [242]. Ausgehend von niedrigen Konzentrationen sagen sie die Erzeugung von Defekten der Art $\left(V_{A l}-O_{N}\right)$ bis zu einer Konzentration von 0.75 at. \% voraus. Bei Konzentrationen, welche diesen kritischen Wert überschreiten, gehen sie zunächst von einer Bildung oktaedrischer $A l(A l-O)$ Defekte aus. Mit einem weiteren Anstieg des Sauerstoffanteils kommt es dann zu einer zunehmenden Verkettung jener, was letztendlich in einer Mikrophasenseparation endet. Bei Sauerstoffkonzentrationen unterhalb der 0.75 at. \% zeigte sich ein linearer Abfall der Rekombinationsenergie von $4.1 \mathrm{eV}$ bei Werten kleiner als 0.1 at. \% auf $3.3 \mathrm{eV}$ beim kritischen Level; einhergehend mit einem Anstieg der Halbwertsbreite. Das beobachtete Verhalten begründen sie mit der Bildung von Clustern bestehend aus Defekten vom Typ $\left(V_{A l}-O_{N}\right)[176]$. Einschränkend ist dieses Modell jedoch nicht im Stande, einen Anstieg der Intensität dieser Lumineszenz um etwa zwei Größenordnungen startend kurz vor der kritischen Konzentration von 0.75 at. \% zu erklären.

Die aktuellste in der Literatur momentan vorhandene experimentelle systematische Untersuchung der Energieniveaus von mit Sauerstoff in Verbindung gebrachten Defekten stammt von der Gruppe um Jiang [23, 32, 119, 177]. Ihre untersuchten mit MOCVD hergestellten $\mathrm{Al}_{x} \mathrm{Ga}_{1-x} \mathrm{~N}$-Filme auf Saphir besitzen verschiedene Aluminiumkonzentrationen von reinem GaN bis hin zum AlN. Aufgrund der Möglichkeit verschiedener stöchiometrischer Zusammensetzungen des $\mathrm{Al}_{x} \mathrm{Ga}_{1-x} \mathrm{~N}$, waren sie in der Lage, der Energieverschiebung der gelben Lumineszenz in GaN $(\sim 2.15 \mathrm{eV})$ bis zum AlN zu folgen, wo diese eine Rekombinationsenergie von $3.90 \mathrm{eV}$ erreicht und somit weit in der UV-Region liegt. Mit ansteigendem Aluminiumanteil beobachteten sie in ihren Proben ab einer Konzentration von $x>0.5$ das Auftauchen eines zweiten Lumineszenzbandes etwa $0.5 \mathrm{eV}$ unterhalb des ersten. Sie schreiben die Lumineszenz 
neu auftretenden Kationleerstellen (siehe Kapitel 2.2.1) zu [177]. Motiviert wird dies durch Berechnungen, welche eine Verringerung der Bildungsenergie jener mit steigendem Aluminiumanteil voraussagen [129,136. Aus diesem Grund muss das Band bei $3.90 \mathrm{eV}$ einen anderen, jedoch zur gelben Lumineszenz in GaN ähnlichen Ursprung aufweisen. Angelehnt an theoretischen Simulationen [129, 243 ordnen sie diesem Band einen DAP-Übergang zwischen einem unbekanntem flachen Donator und einem Komplex der Form $\left(V_{A l}-\right.$ complex $^{2-} \mathrm{zu}$, wobei als mögliche Kandidaten Silizium oder Sauerstoff in Betracht gezogen werden, und in Bezug zu Referenz [243] Sauerstoff favorisiert wird [177]. In weiteren experimentellen Untersuchungen fand die Gruppe um Jiang einen zweiten Lumineszenzpeak mit einer gleichartigen Blauverschiebung von $2.86 \mathrm{eV}$ in $\mathrm{GaN}$ zu $4.71 \mathrm{eV}$ in $\mathrm{AlN}$ [119]. Um eine von der Aluminiumkonzentration $(0<x<1)$ abhängige Beschreibung der Bandlücke für $\mathrm{Al}_{x} \mathrm{Ga}_{1-x} \mathrm{~N}$-Verbindungen $\mathrm{zu}$ erhalten, eignet sich die folgende in zweiter Ordnung gestörte Darstellung [244,245]:

$$
E_{g}(x)=x E_{g}(\mathrm{AlN})+(1-x) E_{g}(\mathrm{GaN})+b x(1-x) .
$$

$E_{g}(\mathrm{AlN})$ und $E_{g}(\mathrm{GaN})$ stehen hierbei für die Größe der Bandlücke der jeweilig angegebenen Verbindungshalbleiter, während $b$ einen sogenannten Beugungsparameter darstellt. Abhängig von den bei der Herstellung vorherrschenden Bedingungen variiert dieser bei den ternären $\mathrm{Al}_{x} \mathrm{Ga}_{1-x} \mathrm{~N}$-Verbindungen zwischen $-0.8 \mathrm{eV}$ bis hin zu $2 \mathrm{eV}$ [245]. Negative Werte treten demnach bei ungenügender kristalliner Qualität auf. Dünne Filme auf kristallfremden Substraten bei denen zuvor eine Nukleationsschicht mit relativ niedrigen Temperaturen aufgebracht worden war, besitzen typischerweise einen Beugungsparameter $b$, welcher positiv und zudem kleiner als $1.3 \mathrm{eV}$ ist [245]. Die Gruppe um Jiang nimmt einen Wert von $b=1 \mathrm{eV}$ und Bandverschiebungen von $70 \%$ und $30 \%$ in $\mathrm{Al}_{x} \mathrm{Ga}_{1-x} \mathrm{~N}$ für das Leitungs- beziehungsweise das Valenzband an [119, 177]. Aufgetragen gegen den Aluminiumanteil $x$ liegen die drei gefundenen Energielevel so jeweils auf horizontalen Linien. Die Autoren sehen dies als einen starken Hinweis dafür, dass die drei tiefen Akzeptorzustände verglichen zum Vakuumenergielevel fest fixiert vorliegen. Aufgrund der vorhandenen Ähnlichkeit ordnen sie das Lumineszenzsignal bei $4.71 \mathrm{eV}$ einem DAP-Transfer zwischen einem unbekanntem flachen Donator und einem Komplex $\left(V_{A l}-\text { complex }\right)^{1-} \mathrm{zu}$, wobei als möglicher Kandidat $\left(V_{A l}-2 O_{N}\right)^{1-}$ angegeben wird [119]. In jüngeren Untersuchungen identifizierte diese Gruppe auch $\sigma$ und $\pi$-Konfigurationen mit energetischen Unterschieden von etwa $0.2 \mathrm{eV}$ [32], jedoch in vertauschter Zuordnung verglichen mit dem Bericht von Pastrňák et al. 197].

Wie anhand der Ergebnisse, insbesondere im Vergleich der Studien der Gruppe um Jiang [32, 119, 177] mit denen von Pastrňák et al. [197], zu erkennen ist, zeigen sich in den Interpretationen der Energieniveaus, welche mit Leerstellen-Sauerstoffkomplexen in Verbindung gebracht werden, signifikante Unterschiede als auch Widersprüche. Pastrňák et al. bestimmten die Lumineszenz mit $\left(V_{A l}-O_{N}\right)$ energetisch höher, als jene mit $\left(V_{A l}-2 O_{N}\right)$, während bei der Gruppe um Jiang ein gegenteiliges Bild 
gezeichnet wird. Im Hinblick auf die Berichte von Youngman und Harris [176, 242] führen höhere Sauerstoffkonzentrationen zu einer Verschiebung des Lumineszenzsignals zu niedrigeren Energien hin, was tendenziell als eine Unterstützung der Theorie von Pastrňák et al. [197] gesehen werden könnte. Jedoch zeigen sowohl Youngman und Harris [176, 242], als auch Jiang et al. [32, 119, 177] deutlich höhere Rekombinationsenergien im Vergleich zu Pastrňák et al. [197]. Möglicherweise lässt sich dies mit der sehr hohen Sauerstoffkonzentration von etwa $(0.7-2.0)$ at. \% in den Proben bei Pastrňák et al. [197] erklären. Nach der Idee von Youngman und Harris müssten bei diesen hohen Sauerstoffanteilen jedoch schon neue Defekttypen wie die oktaedrischen $A l(A l-O)$-Komplexe gebildet und als Ursprung für die beobachtete Lumineszenz mit in Betracht gezogen werden. Ungeachtet dessen existieren in der Literatur zwei Fraktionen, welche entweder Youngman, Harris [176, 242] und Jiang [119, 177] oder Pastrňák et al. [197] folgen, was zu der schon weiter oben angesprochenen breiten energetischen Verteilung der Zuordnungen führt. In Abbildung 2.2 sind die daran beteiligten Übergänge mit zwei farbigen Bereichen gekennzeichnet. Positronen-Annihilationsexperimente sind genau wie schon im Fall von $V_{A l}$ (siehe Kapitel 2.2.1) sensitiv auf Defekte der Form $\left(V_{A l}-\right.$ complex $)$. Aus der aus solchen Experimenten bestimmten Impulsverteilung fanden Mäki et al. [246] $\left(V_{A l}-O_{N}\right)$ als die bevorzugte und damit auch dominierende Form der Aluminimleerstelle in AlN. Bei niedrigen Sauerstoffkonzentrationen $\left(\leq 1 \cdot 10^{18} / \mathrm{cm}^{3}\right)$ gehen sie davon aus, dass nahezu alle Sauerstoffatome innerhalb von AlN in der Struktur $\left(V_{A l}-O_{N}\right)$ vorliegen, was im Einklang mit den weiter oben vorgestellten Theorien liegt. Weitere mit Unterstützung von Positronen-Annihilationsexperimenten durchgeführte Lumineszenzmessungen fanden auch DAP-Übergänge zu $\left(V_{A l}-\text { complex }\right)^{2-}$-Zuständen (siehe Tabelle 2.5 ID 15), was als eine Bestätigung dieser Annahmen gesehen werden kann [52,53].

Theoretische Berechnungen, welche den $\left(V_{A l}-O_{N}\right)$-Komplex untersuchten, ergaben für jenen einen damit konsistenten doppelt negativ geladenen tiefen Akzeptorzustand [100, 127, 129, 163]. Der Komplex $\left(V_{A l}-2 O_{N}\right)$, mit einem energetisch favorisierten Ladungszustand von " $1-$ ", zeigt höhere Bindungsenergien und wird damit als stabiler im Vergleich zu $\left(V_{A l}-O_{N}\right)$ vorausgesagt [100,163. Zusätzlich, wenn ausreichend Sauerstoff innerhalb des Materials vorliegt, zeigt auch der neutrale Ladungszustand des $\left(V_{A l}-3 O_{N}\right)$-Komplex relativ geringe Bildungsenergien [127.

\subsubsection{Kohlenstoff}

Ein zweites zumeist auch ungewollt während des Wachstumsprozesses in AlN eingebrachtes Fremdatom ist Kohlenstoff. Es existieren einige theoretische Simulationen, welche sich mit Kohlenstoff auf dem Aluminium- [96, 97, 99, 123, 126, 130, 148, 165], sowie auch auf dem Stickstoffplatz $96,99,123,148,165]$ beschäftigen. Auf der Position des Aluminiums zeigt Kohlenstoff ein amphoteres Verhalten mit relativ hohen Bildungsenergien, unabhängig von den vorliegenden Wachstumsbedingun- 
gen $(\geq 1 \mathrm{eV})$ 96, 97, 99, 126, 148, 165. Zusätzlich zeigen der neutrale und negative Ladungszustand im Gegensatz zum Positiven eine Art $D X$-Konfiguration im Grundzustand 99, 130, 165]. Hingegen hierzu besitzt Kohlenstoff unter aluminiumreichen Bedingungen und Fermileveln oberhalb der Bandmitte auf der Position des Stickstoffatoms niedrige Bildungsenergien. Dabei ist unter diesen n-Typ-Bedingungen der einfach negative Ladungszustand der energetisch favorisierte 96 99, 148, 165].

Collazo et al. schreiben einen beobachteten Peak in Photolumineszenzmessungen bei $3.9 \mathrm{eV}$ einem direkten Übergang zwischen dem Leitungsband und Kohlenstoff auf der Position des Stickstoffs zu [96]. Hierbei ermittelten sie eine Korrelation zwischen der Intensität des Signals und der Kohlenstoffkonzentration ihrer Proben. Des weiteren berechneten sie die energetischen Positionen unter der Nutzung einer HSE-Methode, welche mit einem in Absorptionsmessungen gefundenen Signal bei $4.7 \mathrm{eV}$ korrespondiert. Die dabei vorausgesagte minimale Emissionsenergie für diesen Übergang liegt bei $3.5 \mathrm{eV}$ und somit sehr nahe zur beobachteten Position des experimentellen Signals. Dieser Peak zeigt sich jedoch nur in Proben, in denen sich der Anteil an Kohlenstoff deutlich über den Konzentrationen von anderen Fremdatomen befindet 96 (beispielsweise Silizium 98]). Der hier vorgestellten Interpretation von Collazo et al. folgend existieren weitere Veröffentlichungen, welche jenes Signal Kohlenstoff zuordnen (siehe Tabelle 2.5 ID 12). Ein Signal bei $2.8 \mathrm{eV}$ wurde von Gaddy et al. zuletzt einem DAP-Übergang zwischen der Stickstoffleerstelle und Kohlenstoff auf der Stickstoffposition zugeordnet 97,98. Dabei berufen sie sich auf selbst durchgeführte HSE-Simulationen, bei denen kein Kohlenstoffkomplex allein zur beobachteten Energie korrespondierte. Sie unterstützen diese Zuordnung weiterhin mit der Kombination ihrer Ergebnisse mit früher durchgeführten Absorptions- 96 und kohlenstoffkonzentrationsabhängigen Messungen [173]. Zudem widersprechen sie aktiv den Ergebnissen von Sedhain et al. [67], die diesen Peak Aluminiumleerstellen zuordnen. In Proben, welche zusätzlich zu Kohlenstoff auch noch mit Silizium kodotiert wurden, fanden Gaddy et al. einen Lumineszenzpeak bei $4.3 \mathrm{eV}$ [98]. Diese Rekombination schreiben sie einem Transfer zwischen dem Leitungsband und dem $\left(C_{N}-S i_{A l}\right)$-Komplex zu. Im zugehörigem Bericht fanden sie mit Hilfe von theoretischen Simulationen eine kleine Region in der Nähe der Bandmitte, bei welcher die Bildungsenergie für jenen Komplex unter der der jeweils einzelnen Anteile liegt. Verglichen zu einer früheren Veröffentlichung dieser Autoren [97] liegt die Bildungsenergie hier für Kohlenstoff auf der Stickstoffposition im Fall von aluminiumreichen Bedingungen etwa $1 \mathrm{eV}$ höher. Unter einer Annahme jener niedrigerer Energie für $C_{N}$ würde sich die schmale zuvor favorisierte Region für $\left(C_{N}-S i_{A l}\right)$ jedoch schließen. In der Literatur werden ab und zu Lumineszenzpeaks um $4.4 \mathrm{eV}$ Kohlenstoff zugesprochen [48,74, 114]. Jedoch zeigt sich häufig keine Korrelation zwischen jenen Peaks und der Kohlenstoffkonzentration im Material [45,247, 248]. Eine alternative Zuordnung des $4.7 \mathrm{eV}$-Absorptionsbandes und den dazugehörigen Emissionen wurde von Irmscher et al. vorgeschlagen [249]. Sie präparierten ihre Proben neben ${ }^{12} \mathrm{C}$ auch mit ${ }^{13} \mathrm{C}$-Isotopen und untersuchten diese mit einer Spektroskopie der lokalen Schwin- 
gungsmoden (LVM), im Kontext von zusätzlichen Absorptionsmessungen. Aufgrund kleiner energetischer Verschiebungen der Schwingungsmoden, hervorgerufen durch die unterschiedlichen Massen der Isotope, konnten sie ihre LVM-Spektren einem Kohlenstoffdefekt bestehend aus drei Kohlenstoffatomen zuordnen. Bei niedrigen Sauerstoffanteilen fanden sie den Ladungszustand "1-" als energetisch favorisierten vor. Mit einer ansteigenden Sauerstoffkonzentration erklären sie das Verschwinden des zuvor beobachteten Absorptionspeak mit einem damit einhergehendem Anstieg des Fermilevels und einer Stabilisierung des "2-" Ladungszustandes. Jener lässt im betrachtetem Energiebereich jedoch keine Absorption erwarten.

\subsubsection{Metalle}

Ein untersuchtes Materialsystem, ursprünglich in der Herstellung patentiert als rot-leuchtendes Luminophor für technische Anwendungen [16], ist mangandotiertes AlN. Karel et al. fanden in diesem System eine Lumineszenz um $2.08 \mathrm{eV}$, welche eine stark ausgeprägte Feinstruktur aufweist [212, 213, 250]. Die einzelnen Peaks haben dabei Abstände einer Phononenenergie von $23 \mathrm{meV}$ [213] und werden mit lokalen Schwingungen erklärt. In zeitabhängigen Messungen fanden sie Lebensdauern im Bereich um $1 \mathrm{~ms}$ 212 und sprechen jene Lumineszenz dem $M n^{4+}$-Zustand auf einem substitutionellen Aluminiumplatz zu. Aufgrund fehlender paramagnetischer Resonanz wurde der bivalente Zustand von Mangan ausgeschlossen [213]. Mit Diffusionsexperimenten konnten dabei Konzentrationen von bis zu 0.1 at. \% eingebracht werden. Wenn der Magnesiumanteil einen Wert von 1 at. \% überschritt, kam es in den dort untersuchten Proben zu einer Löschung der Lumineszenz. Basierend auf den Ergebnissen von Karel et al. wird in der Literatur typischerweise die tetravalente Konfiguration als Zustand für die in diesem Bereich beobachtete Lumineszenz angenommen [24, 170, 183, 193, 198, 199, 215, 216, 218, 251]. Im Widerspruch hierzu stehen jedoch Untersuchungen von Miyajima et al., bei denen mit Hilfe von Röntgenabsorptionsspektroskopie mit einem Vergleich zu bekannten Ladungszuständen in anderen Manganverbindungen ein Zustand zwischen " $2+$ " und " $3+$ " in AlN ermittelt wurde [210].

Mit Eisen versetzte Proben zeigen einen scharf ausgeprägten Lumineszenzpeak bei $1.3 \mathrm{eV}$ mit einem Akzeptorniveau bei etwa $3 \mathrm{eV}$ 230]. Der Übergang wird dabei einem Transfer vom ${ }^{4} T_{1}$ zum ${ }^{6} A_{1}$-Level des $F e^{3+}$ zugeschrieben. EPR-Experimente deuten auch auf bivalentes Eisen mit einem erwarteten Akzeptorzustand um $3.96 \mathrm{eV}$ oberhalb des Valenzbandmaximums hin [140]. Aufgrund der großen Bandlücke von AlN verglichen $\mathrm{zu}$ vielen anderen III-V-Halbleitern befindet sich dieser angeregte Zustand ebenfalls innerhalb der Bandlücke [140,230.

Das Einbringen von Chrom in AlN führt zu zwei scharfen Bändern bei $1.813 \mathrm{eV}$ und $1.808 \mathrm{eV}$, welche jeweils den $R^{2}$ und $R_{2}$ - ̈̈bergängen vom ${ }^{2} E$ zum ${ }^{4} A_{2}$-Zustand des $C r^{3+}$ zugeordnet wurden 201]. Ein weiterer ebenfalls scharf ausgeprägter Peak 
mit leicht erniedrigter Energie bei $1.77 \mathrm{eV}$ wird Clustern von $\mathrm{Cr}^{3+}$ auch bekannt aus anderen Wirtsgittern zugeordnet 201.

Zusätzlich präparierte die selbe Gruppe einige Proben mit Kupfer und beobachtete daraufhin eine Lumineszenz um $2.95 \mathrm{eV}$. Eine Kodotierung dieser amorphen Proben mit zusätzlichem Mangan und Terbium führte zu einer starken Rotverschiebung des Kupfersignals zu etwa $2.3 \mathrm{eV}$, was von den Autoren mit einer Nebenaktivierung gegebenenfalls mit einer Bildung von Mangan-Kupfer-Paaren erklärt wird 201].

Yoshida et al. fanden ein Lumineszenzsignal bei $2.43 \mathrm{eV}$ in mit Niob dotierten Proben, jedoch ohne dies genauer zu spezifizieren [183].

\subsubsection{Akzeptoren und Donatoren}

Neben der ungewollten Inkorporation von Fremdatomen führt auch die Dotierung mit Akzeptoren oder Donatoren mit dem Ziel einer Steigerung der elektrischen Leitfähigkeit zu verschiedenen Defektbändern. Einige dieser Lumineszenzen sind dabei Magnesium zugeordnet $[19,22,23,150$ (siehe Tabelle 2.5 ID 1, 3 und 5). Die Gruppe um Jiang beobachtete in Mg-dotierten Filmen Rekombinationen bei $5.5 \mathrm{eV}, 5.4 \mathrm{eV}$ und $4.7 \mathrm{eV} 22,150$. Sie ordnen jene Übergänge jeweilig einem Transfer vom Leitungsband oder einem flachen Donator, der $V_{N}^{1+}$ und als drittes der $V_{N}^{3+}$ Leerstelle zu einem substitutionellen Magnesium auf einem Aluminiumplatz zu. In temperaturabhängigen Lumineszenzmessungen zeigt der Peak bei $4.7 \mathrm{eV}$ eine Aktivierungsenergie von $0.5 \mathrm{eV}$ [150], was gut $\mathrm{zu}$ den theoretischen Ergebnissen des Magnesiumlevels in AlN [124, 126, 136, 143, 145 149] und der energetischen Position von $V_{N}^{3+}[25$ korrespondiert. Zusätzlich zeigen auch temperaturabhängige Leitfähigkeitsmessungen eine Aktivierungsenergie von $0.5 \mathrm{eV}$ [22]. Die Zuordnung der Signale bei $5.4 \mathrm{eV}$ und $4.7 \mathrm{eV}$ zu DAP-Übergängen ausgehend von Stickstoffleerstellen, welche wie schon in Kapitel 2.2.1 erwähnt als Kompensationszentren agieren können, wurde von den Autoren durch eine starke Korrelation zwischen der elektrischen Leitfähigkeit und der Intensität der Lumineszenz bei $4.7 \mathrm{eV}$ motiviert [22]. Typisch für DAP-Rekombinationen zeigen diese in den Proben lange Lebensdauern im Bereich von Mikrosekunden [22] (siehe Kapitel 2.2.).

Basierend auf den Ergebnissen mit Magnesium wurden auch dünne AlN-Filme mit Zink dotiert und Lumineszenzbänder in einer gleichartigen Weise beobachtet und interpretiert (siehe Tabelle 2.5 ID 2 und 7). Hierbei fanden Nepal et al. zwei Lumineszenzsignale bei $5.4 \mathrm{eV}$ sowie $4.5 \mathrm{eV}[21]$. Analog zum vorherigem Fall bei Magnesium ordneten sie den energetisch höheren Übergang einem direkten Transfer vom Leitungsband zur Zinkstörstelle zu. Unterstützt wird diese Annahme durch eine zum Zink passende Aktivierungsenergie und eine kurze Rekombinationslebensdauer von 200 ps [21]. Auch der zweite Lumineszenzpeak, zugeordnet zu einem DAPÜbergang mit $V_{N}^{3+}$, stimmt mit der vorherig bestimmten Aktivierungsenergie gut überein und besitzt eine Lebensdauer im Mikrosekunden-Bereich [21]. Des weiteren 
wurde auch in anderen Experimenten eine vom Zinkanteil abhängige Intensität des Signals beobachtet 169].

Frühe Untersuchungen von siliziumdotiertem AlN mit einer Quecksilberlampe und Kathodolumineszenz wurden schon von Sensse in den späten 20er Jahren des letzten Jahrhunderts durchgeführt [15]. Mit Siliziumkonzentrationen zwischen $0.08 \%$ wt. und $0.5 \%$ wt. beobachtete er blaue Phosphoreszenz mit Lebensdauern im Bereich einer Minute bei Raumtemperatur. Die Proben zeigten hierbei effektive Anregungszentren um $270 \mathrm{~nm}, 280 \mathrm{~nm}$ und $290 \mathrm{~nm}$. Viel später wurde in Proben, welche auf Siliziumsubstraten aufgebracht wurden, ein Signal bei $3.9 \mathrm{eV}$ einem Übergang zwischen Silizium und dem Valenzband zugeordnet (siehe Tabelle 2.5 ID 11). Mit zunehmender Dicke der aufgebrachten Schicht AlN verschwindet das Lumineszenzsignal und ist in auf Saphir aufgewachsenen Proben von vornherein nicht präsent [174].

\subsubsection{Europium}

Da im Rahmen der hier vorliegenden Arbeit auch Proben, welche mit Europium dotiert sind, exemplarisch untersucht wurden, wird im folgenden Abschnitt eine kurze Darstellung des beobachtbaren Lumineszenzverhalten jenes in AlN gegeben. Bei Europium handelt es sich um ein Element der sogenannten seltenen Erden mit einer im neutralen Zustand äußeren Elektronenkonfiguration von $4 f^{7} 5 s^{2} 5 p^{6} 6 s^{2}$ [252]. Eingebracht in AlN kann es im Ladungszustand " $2+$ " durch Abgabe der beiden 6s-Elektronen oder im Zustand "3+" durch zusätzliche Abgabe eines Elektrons aus der $4 f$-Schale vorliegen. Das daraus mit optischer oder elektronischer Anregung resultierende Lumineszenzverhalten ist dabei von sehr unterschiedlicher Natur. Im Fall von $E u^{3+}$ ist dabei ein Spektrum mit einer Vielzahl von scharf ausgeprägten Übergängen von den Zuständen ${ }^{5} \mathrm{D}_{0}$ und ${ }^{5} \mathrm{D}_{1}$ zu den Niveaus ${ }^{7} \mathrm{~F}_{0-4}$ zu beobachten 228], wobei der Übergang ${ }^{5} \mathrm{D}_{0} \rightarrow{ }^{7} \mathrm{~F}_{2}$ das Spektrum bei einer in der Literatur durchschnittlich angegebenen Energie von 1.993(15) eV (siehe Tabelle 2.5 ID 39) dominiert. Verglichen dazu werden Lumineszenzen bei $2.65(5) \mathrm{eV}$ oder $2.26(4) \mathrm{eV}$ (siehe Tabelle 2.5 ID 30 und 35) dem divalenten Zustand mit Übergängen aus dem $4 f^{6} 5 d$ in das $4 f^{7}$ Elektronensystem in jeweilig strukturell unterschiedlichen Umgebungen zugeschrieben. Die Konzentration des Europium ist dabei in ihrer Verteilung nicht unbedingt homogen. So wurden sowohl bei $E u^{3+}$ angereicherte Gebiete an Korngrenzen [253] als auch bei $E u^{2+}$ unterschiedliche Verteilungen in Partikeln mit blauer Lumineszenz im Inneren und grüner am Rand 203, 208 beobachtet. Unabhängig vom Ladungszustand wird sowohl für $E u^{3+} 220,252,254$ als auch $\mathrm{Eu}^{2+}$ 207] eine substitutionelle Position an Stelle des Aluminiums in AlN begründet auf experimentellen Messungen angenommen. Auch in Simulationen zeigt sich $E u^{3+}$ in dieser Positionierung stabil [132]. Mit zunehmender Inkorporation des seltenen Erdmetalls ist zunächst ein Dichteanstieg [253], sowie auch eine Vergrößerung der Gitterparameter zu beobachten [207]. Bei sehr hohen Konzen- 
trationen $\left(\mathrm{Eu}_{2} \mathrm{O}_{3}>5 \%\right.$ wt. $)$ reduziert sich diese jedoch wieder [253]. Im Fall von $E u^{2+}$ führt die Zugabe von Silizium [204] als auch eine möglichst hohe Temperatur bei der Herstellung von Pulverproben [204, 205, 208] zu einem deutlichen Anstieg der Lumineszenzintensität. Ohne Silizium zeigt sich nach Inoue et al. [205] im divalenten Zustand ein verstärkt grünes Leuchtverhalten durch eine mögliche andere Phase, wohingegen mit dessen Zusatz ein Lumineszenzspektrum im blauen Bereich beobachtet werden kann. Auch in Proben mit erhöhtem Sauerstoffanteil ist eher eine Lumineszenz im blauen Bereich sichtbar [207]. Hoch aufgelöste DunkelfeldRastertransmissions-Elektronenmikroskopie-Messungen zeigen in siliziumdotierten Proben die Konfiguration monoatomarer Lagen von $E u^{2+}$ [206]. Zudem ist eine Anreicherung von Silizium um diese Lagen herum zu beobachten, welche zu einer Verringerung der Gitterkonstanten und einer damit erst möglichen Bildung jener Strukturen einhergeht. Des weiteren tritt auch zwischen diesen Schichten jeweils ein mit Silizium angereicherter Bereich auf, welcher den Autoren zufolge zu einer Polarisationsinversion im AlN führt 206]. Die Koordination erfolgt dabei unregelmäßig ohne einen Hinweis auf die Bildung eines Übergitters.

Die Europiumatome werden häufig über Ionenimplantation in das AlN eingebracht. Ein anschließendes Ausheilen der Proben bei niedrigen Temperaturen $\left(<1500^{\circ} \mathrm{C}\right)$ repariert nur einen kleinen Teil der während der Implantation entstandenen Defekte, führt jedoch zu einem starken Anstieg des Lumineszenzsverhaltens 226, 227, 254, 255]. 1 Durch sogenanntes Channelling (Implantation in Richtung der $c$-Achse) kann die Defekterzeugung von vornherein gemindert werden, was grundsätzlich in einer stärker ausgeprägten Lumineszenz resultiert [226]. Eine Kodotierung von Eu ${ }^{3+}$ implantierten Proben mit verschiedenen anderen Ionen $(\mathrm{F}, \mathrm{Cl}, \mathrm{Ne}$ ) führt lediglich zu einer zusätzlichen Abschwächung der sichtbaren Rekombinationen, jedoch nicht zu einer weiteren Aufspaltung in neue Starklevel [185]. Ein Ausheilen bei sehr hohen Temperaturen zwischen $1900-2100^{\circ} \mathrm{C}$ kann Yin et al. zufolge zudem eine Umwandlung von $E u^{3+}$ in $E u^{2+}$ mit sich bringen. Das Lumineszenzverhalten bei temperaturabhängigen Messungen deutet auf eine geringe Kopplung zwischen den Phononen in AlN und den $4 f$-Elektronen des $E u^{3+}$ hin [225]. Eine Reduzierung der Lumineszenzintensität durch thermische Löschung tritt auch noch bei Temperaturen oberhalb von Raumtemperatur meist nur geringfügig auf [205, 225]. Lediglich bei implantierten Dünnschichtproben von Rodrigues et al. 257 zeigt sich eine etwaige Halbierung der Intensität bei Raumtemperatur verglichen zu Messungen bei $14 \mathrm{~K}$.

Nach einem Modell von Lozykowski und Jadwisienczak [225, 252] bilden die seltenen Erden in AlN isovalente Zentren, welche attraktiv auf Löcher wirken. Nach Einfang eines solchen kann jenes aufgrund der coulombschen Wechselwirkung im Anregungsfall auch noch ein freies Elektron aquirieren und somit eine Umwandlung

\footnotetext{
${ }^{1}$ Ausgenommen sei hier ein in der Literatur dargestellter Fall, bei dem in nanokristallinen Filmen von einer Verringerung der Leuchtintensität bei Temperaturen oberhalb von $900^{\circ} \mathrm{C}$ berichtet wird 256.
} 
in ein isovalent gebundenes Exziton vollführen. Jene besitzen demnach eine deutlich höhere Lebensdauer (einige 100 ns bis einige 1000 ns [252]) als zu neutralen Donatoren oder Akzeptoren gebundene Exzitonen (siehe hierzu Kapitel 2.1.3). Dem Modell zufolge findet frühzeitig ein Augertransfer vom Exziton in das $4 f$-Elektronensystem des seltenen Erds statt, wodurch keine optische Abregung des isovalent gebundenen Exzitons beobachtet werden kann. Nach einer möglichen vorherigen thermischen Relaxation des angeregten Elektrons ist schlussendlich ein strahlender Übergang in eines der niedriger angeregten Niveaus oder in den Grundzustand möglich. Des weiteren gehen Lozykowski und Jadwisienczak von einem statistisch überproportionalen Auftreten von seltenen Erd-Dimeren oder auch -Trimeren aus, zwischen welchen es zu Energietransferen kommen kann 225]. Begründet wird dieses Verhalten durch die Beobachtung von bis zu drei Anregungsbändern bei Photolumineszenz-AnregungsMessungen [225, 229, 257], welche diesen Trimeren und Dimeren zugeordnet werden könnten [225]. Eine mögliche Löschung des angeregten Zustandes erfolgt der Theorie nach durch thermische Ionisation, zusätzlichen Locheinfang oder thermische dissoziation des Exzitons, Verlust durch Augertransfer in ein anderes Zentrum oder aber auch durch eine thermische Relaxation in den Grundzustand über Phononen (im Fall von $\mathrm{Eu}^{3+}$ eher unterdrückt durch großen energetischen Abstand) [225, 252]. Demgegenüber steht eine Theorie von Dorenbos und van der Kolk [258], nach welcher sich $\mathrm{Eu}^{3+}$ als Elektronenfalle verhält und eine thermische Löschung durch einen Energietransfer zurück zu einem gebundenen Exziton auszuschließen ist.

Neben stationären Lumineszenzmessungen von Europium existieren auch einige Berichte zu zeitabhängigen Versuchsreihen [220, 221, 259, 260]. Vetter et al. [259] als auch Jadwisienczak et al. [220] beobachten dabei im Fall von $E u^{3+}$ einen nahezu exponentiellen Zerfall mit Lebensdauern um $360 \mu$ s beziehungsweise $420 \mu$ s. Hingegen zeigen sich bei Liu et al. 221] überlagerte Zerfallstransienten mit deutlich kürzeren Lebensdauern von jeweils 38(1) $\mu$ s und 161(2) $\mu$ s, was auf zwei verschiedene Konfigurationen aufgrund unzureichender kristalliner Qualität der Proben hindeuten könnte. Für $E u^{2+}$ existiert ein Bericht von Yin et al. [260] in welchem auch ein doppelt exponentieller Zerfall mit jeweiligen Lebensdauern unterhalb von $10 \mu \mathrm{s}$ beobachtet wird. Durch eine Zugabe von $\mathrm{CaF}_{2}$ während des Wachstums erhöhen sich diese jedoch auf etwa $39 \mu$ s und $287 \mu$ s.

Neben den Berichten von Europium in AlN wurde in der Literatur auch die ternäre Verbindung $\mathrm{Al}_{x} \mathrm{Ga}_{1-x} \mathrm{~N}$ mit unterschiedlichen stöchiometrischen Anteilen $x$ mit Europium dotiert und optisch untersucht [222, 223, 261]. Dabei zeigt sich das stärkste Lumineszenzverhalten des $E u^{3+}$ bei einer Zusammensetzung zwischen $x=0.3[223]$ und $x=0.5[222$, welches im Vergleich zu GaN einem Intensitätszuwachs um den Faktor 100 entspricht 222]. Mit sich ändernder Komposition ergibt sich eine energetische Rotverschiebung des gesamten Lumineszenzspektrums um $10 \mathrm{meV}$ [222] respektive $7 \mathrm{meV}$ [261] von GaN zu AlN, wobei eine asymmetrische Variation der Linienbreite mit sich ändernder Zusammensetzung zu beobachten ist [261]. Dies deutet für Wang et al. auf eine exzitonische Natur der Übergänge hin [261]. Un- 
abhängig davon, ob oberhalb oder unterhalb der Bandlücke angeregt wird, zeigt sich ein ähnliches Lumineszenzverhalten für Proben mit $x>0.15$ [261]. Generell ergeben sich auch in AlN nahezu gleichartige $E u^{3+}$-Spektren bei Kathodolumineszenzals auch Photolumineszenzmessungen [225]. Als alternativer Ursprung für die in Photolumineszenz-Anregungs-Messungen stark ausgeprägten Anregungsbänder kann nach Wang et al. auch ein Ladungstransfer in die $5 d^{1}$-Konfiguration des seltenen Erds in Frage kommen [261]. Dieser Zustand ist demnach in den verschiedenen Spinzuständen um $1 \mathrm{eV}$ aufgesplittet, wodurch das höhere Anregungsband erst bei einer Komposition von $x>0.6$ innerhalb der Bandlücke liegt. 


\section{Experimenteller Aufbau}

Im Rahmen dieser Arbeit wurde ein Analysesystem für die Kurzzeit-LumineszenzSpektroskopie von Festkörpern als auch Flüssigkeiten im sichtbaren und nahen UV- sowie Infrarotbereich aufgebaut, in Betrieb genommen und auch erweitert. Die vorhandene Vakuumkammer [259], wie auch ein Spektrometer für zeitunabhängige Messungen wurden um ein Femtosekunden-Lasersystem sowie eine für zeitaufgelöste optische Messungen spezialisierte Streak-Kamera ergänzt. Eine schematische Darstellung ist in Abbildung 3.1 gegeben.

Im folgenden Kapitel werden die einzelnen neu hinzu gekommenen Komponenten des Versuchsaufbaus in ihrer Funktionsweise als auch in ihren spezifischen Möglichkeiten/Limitierungen detailliert beschrieben. Auf die Vakuumkammer und das Spektrometer für zeitunabhängige Messungen wird nur kurz eingegangen, da für diese schon andernorts längere Ausführungen bestehen [259].

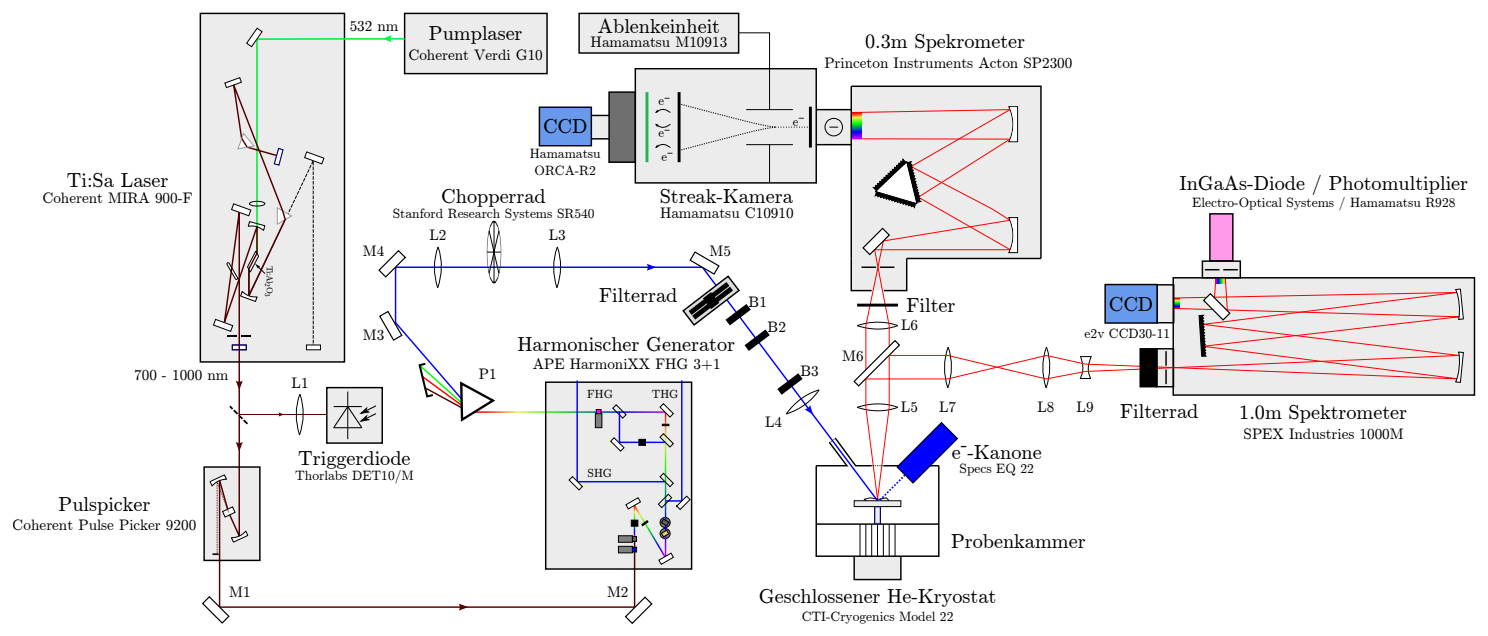

Abbildung 3.1: Schematische Darstellung des kompletten Lumineszenzaufbaus mit Femtosekunden-Lasersystem (links), Probenkammer (Mitte unten) und zweigeteilter Analysemöglichkeit jeweils für zeitabhängige (Mitte oben) oder spektral hochaufgelöste Messungen (rechts). Gezeigt ist hier die Konfiguration für die vierte harmonische Anregung (3+1) der fundamentalen Wellenlänge zeitlich separiert über den Pulspicker. 


\subsection{Lasersystem}

Zur optischen Anregung der Photolumineszenzmessungen wird ein durchstimmbares Femtosekunden-Lasersystem verwendet. Die zentrale Komponente besteht aus einem durch einen Halbleiterlaser gepumpten $\mathrm{T} i \mathrm{Al}_{2} \mathrm{O}_{3}$-Laser, welcher sich über einen Wellenlängenbereich von 700 - $1000 \mathrm{~nm}$ modengekoppelt betreiben lässt. Des weiteren befindet sich ein Pulspicker zur Selektion einzelner Pulse als auch ein harmonischer Generator zur Erzeugung von Wellenlängen im blauen sowie nahen UV-Bereich im Strahlengang.

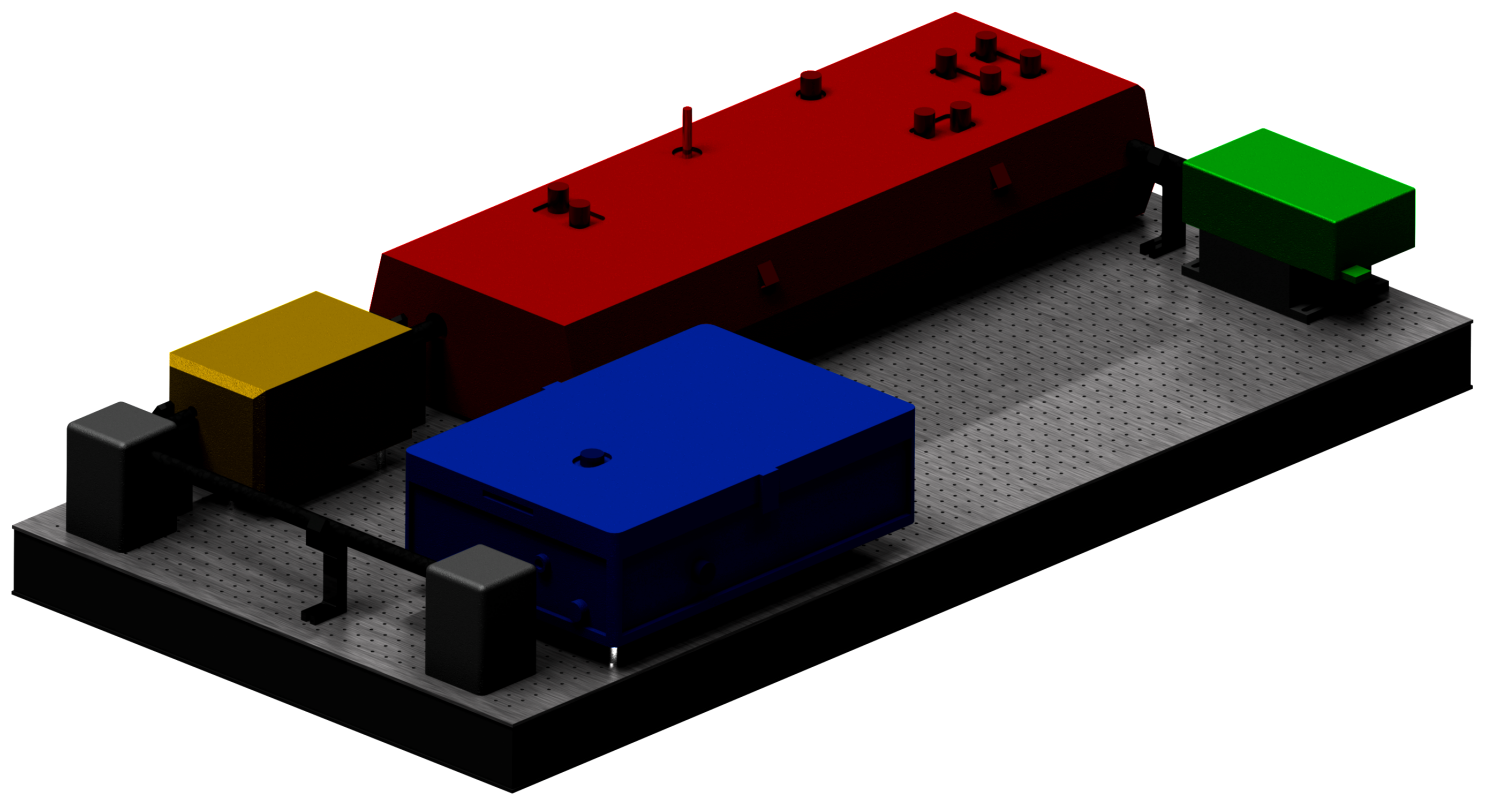

Abbildung 3.2: Lasersystem zur optischen Anregung ohne äußere Schirmung. Der Femtosekunden-Laser Mira Model 900-F (rot) gepumpt durch den Verdi G10 (grün) gefolgt vom Pulspicker Model 9200 (gelb) und dem harmonischen Generator HarmoniXX FHG 3+1 (blau).

\subsubsection{Pumplaser}

Bei dem im Versuchsaufbau für den Femtosekunden-Laser genutzten Pumpmechanismus handelt es sich um einen optisch gepumpten Halbleiterlaser. Gepumpt wird das aktive Medium über eine Laserdiode mit einer Wellenlänge von $808 \mathrm{~nm}$ [262]. Die Laserkavität besteht aus einem mit einer teils lichtdurchlässigen Begrenzungsschicht abgeschlossenen Anordnung von Quantentöpfen (Neodym-dotiert), welche das eintreffende Pumplicht absorbieren, und einen um die Kavität auf der gegenüberliegenden Seite abschließenden hochreflektiven Bragg-Spiegel [263] (siehe Abbildung 3.3). Das in dieser Kavität erzeugte Laserlicht besitzt eine Wellenlänge von $1064 \mathrm{~nm}$. Mit 
Hilfe eines frequenzverdoppelnden Kristalls (siehe hierzu Kapitel 3.1.4) emittiert der Halbleiterlaser Licht einer Wellenlänge von $532.2 \mathrm{~nm}$, was gesehen auf das Absorptionsverhalten des $\mathrm{Ti}: \mathrm{Al}_{2} \mathrm{O}_{3}$-Kristalls im Femtosekunden-Laser einer nahezu optimalen Anregungswellenlänge entspricht. Auskunft über die Strahlqualität kann die Beugungsmaßzahl [264], welche ein Maß für das Divergenzverhalten darstellt, geben. Sie wurde bei diesem Laser auf $M^{2}=1.01$ sowohl in horizontaler als auch vertikaler Richtung bestimmt. Die Laserleistung lässt sich durch den Pumpstrom und damit der Pumpleistung der Laserdiode nahezu stufenlos zwischen 0.04-10 W am zugehörigen Controller regulieren, wobei Anzeige und tatsächlich abgegebene Leistung exakt übereinstimmen. Der Diodenstrom bei $10 \mathrm{~W}$ Ausgangsleistung beträgt hierbei $40.7 \mathrm{~A}$.

Abbildung 3.3: Schematischer Aufbau des Verstärkermediums des Verdi G10. Die nur $10 \mu \mathrm{m}$ dicke Anordnung aus Quantentöpfen und Bragg-Spiegel ist direkt mit einem aktiv durch Wasser gekühlten Kühlkörper verbunden (Abbildung nach $[262]$ ).

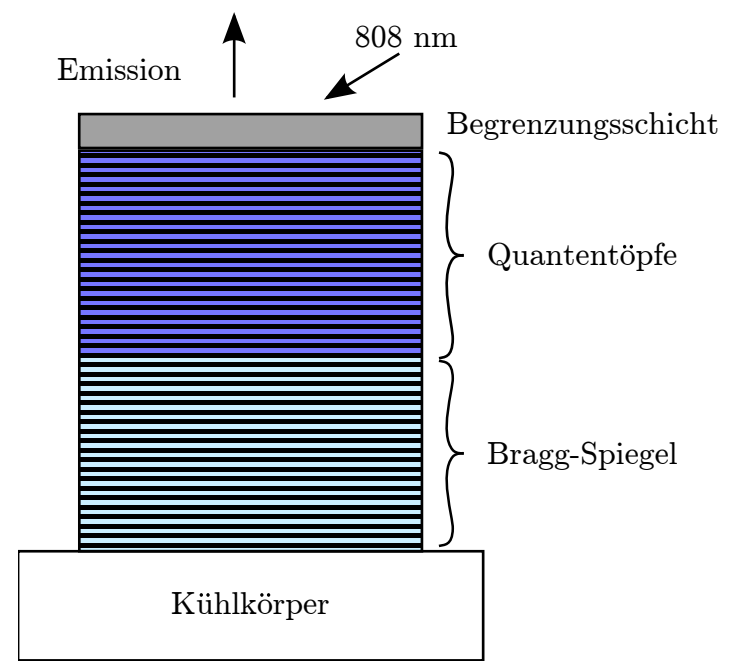

\subsubsection{Titan-Saphir Laser}

Die zur Ultra-Kurzzeitspektroskopie benötigten Femtosekunden-Pulse werden über einen Titan-Saphir-Laser erzeugt. Der erste auf $\mathrm{Ti}: \mathrm{Al}_{2} \mathrm{O}_{3}$ basierende Laser wurde 1982 vorgestellt [265]. Für eine detaillierte Beschreibung des Niveausystems sei auf das Kapitel A im Anhang verwiesen. Bei dem in diesem experimentellen Aufbau verwendeten System handelt es sich um einen Mira Model 900-F der Firma Coherent, Inc. Die Anregung des Lasers erfolgt über den in Kapitel 3.1.1 beschriebenen Verdi G10 bei einer Wellenlänge von $532.2 \mathrm{~nm}$. Dieser wird über ein Justagesystem innerhalb des Mira, bestehend aus vier Spiegeln und einer Linse, durch einen für sichtbares Licht transparenten Infrarotspiegel auf den in der Mitte des Laserkopfes befindlichen $\mathrm{T} i: \mathrm{Al}_{2} \mathrm{O}_{3}$-Kristall fokussiert. Die eigentliche Kavität des FemtosekundenLasers mit einer Länge von etwa $2 \mathrm{~m}$ besteht aus sieben Spiegeln (siehe Abbildung 3.4 M1 - M7), bei welchen es sich bis auf bei M4 und M5 um plane Infrarotspiegel mit einem hohen Reflexionsindex zwischen 700 - 1000 nm handelt. M4 und M5 sind 
gewölbte Spiegel mit einem Radius von $10 \mathrm{~cm}$ zur Fokussierung des Lichts auf den in deren Mitte befindlichen Kristall. Der für die Ultrakurzzeit-Experimente

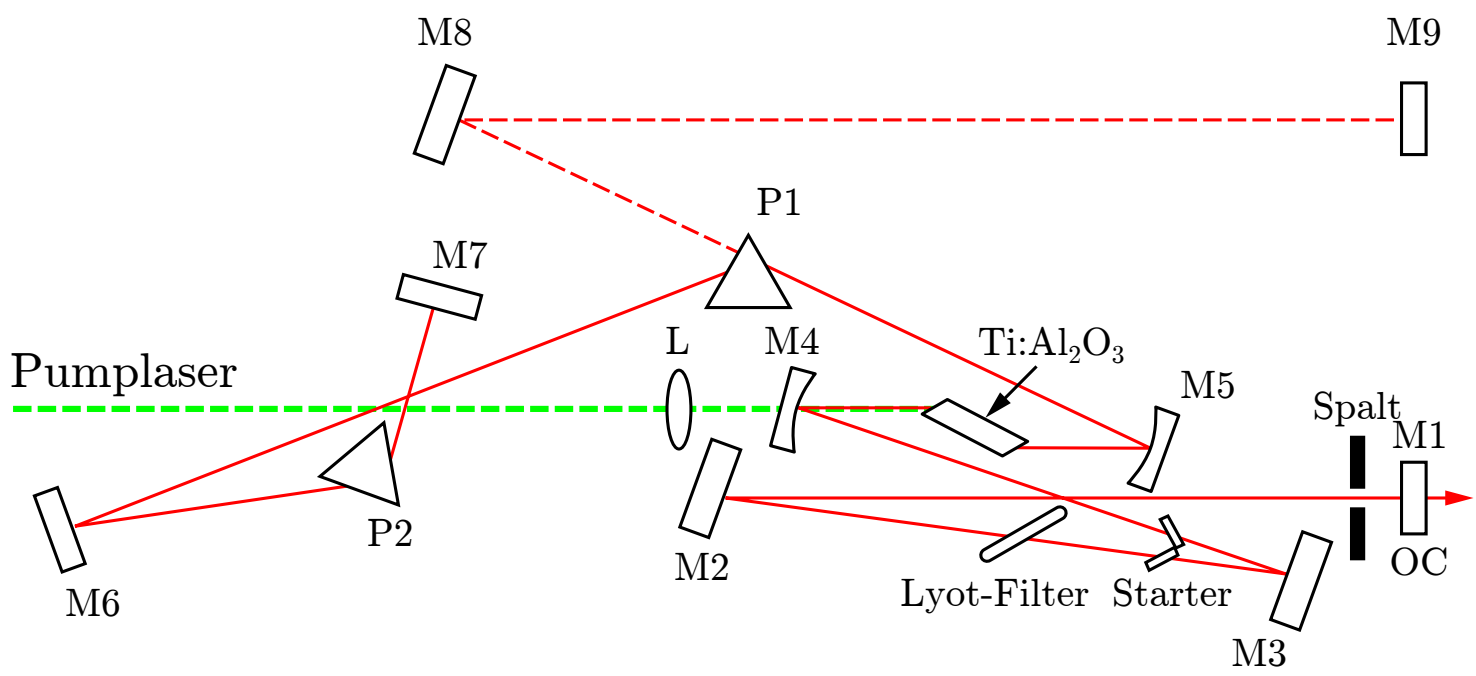

Abbildung 3.4: Kavität des Mira Femtosekunden-Lasers. Spiegel M8 und M9 werden für die Justage bei der Einrichtung, wie auch im Falle von Leistungsoptimierungen benötigt. Weiterhin kommen sie auch in der alternativen Kavität für den Pikosekundenbetrieb zum Einsatz. Das Spiegelsystem des Pumpstrahles innerhalb des Laserkopfes ist hier nicht dargestellt. Die Abbildung wurde abgewandelt aus 266 übernommen.

benötigte modengekoppelte Betrieb der Kavität wird im Mira durch ein sättigbares Absorbersystem realisiert. Dieses zweiteilige System basiert auf einer Komponente, welche lediglich hohe intensive Laserpulse fokussiert und verstärkt sowie einer zweiten, die zur Abschwächung des Dauerstrichanteils beiträgt. Die fokussierende und damit Dauerstrich- und gepulste Anteile trennende Komponente ist der $\mathrm{Ti}: \mathrm{Al}_{2} \mathrm{O}_{3}$-Kristall selbst, fungierend als Kerr-Linse. Hierbei macht man sich den elektrooptischen KerrEffekt 267] zu nutze, der eine Änderung des Brechungsindexes $\Delta n$ in Abhängigkeit der Intensität $I$ des transferierenden Lichts selbst beschreibt [268]:

$$
\Delta n=n_{0}+\gamma I .
$$

Der Kerr-Koeffizient von Saphir besitzt bei $800 \mathrm{~nm}$ einen Wert von $\gamma=3.1 \times$ $10^{-16} \mathrm{~cm}^{2} / \mathrm{W}$ [269] und führt somit bei steigender Intensität zu einer stärkeren Brechung im Kristall. Das Strahlprofil weist zur Mitte hin eine höhere Intensität auf, wodurch sich ein gradueller Brechungsindex innerhalb des $\mathrm{Ti}: \mathrm{Al}_{2} \mathrm{O}_{3}$-Kristalls ausbildet, der je nach Lichtleistung zu einer unterschiedlich starken Fokussierung führt. Da im modengekoppelten verglichen zum Dauerstrichbetrieb eine wesentlich höhere Leistungsdichte vorliegt $\left(3 \times 10^{7} \mathrm{~W} / \mathrm{cm}^{2}\right.$ Peak-Leistung $\left.270 \mathrm{zu} 2 \times 10^{2} \mathrm{~W} / \mathrm{cm}^{2}\right)$, resultiert dies in einem reduzierten Strahldurchmesser der gepulsten Leistung gegenüber 
dem Dauerstrichanteil (siehe Abbildung 3.5 (a)). Aufgrund der unterschiedlichen lateralen Ausdehnung kann der Dauerstrichanteil nun gezielt unter Zuhilfenahme eines größenverstellbaren Spalts innerhalb der Kavität stark gedämpft werden (siehe Abbildung 3.5 (b)).

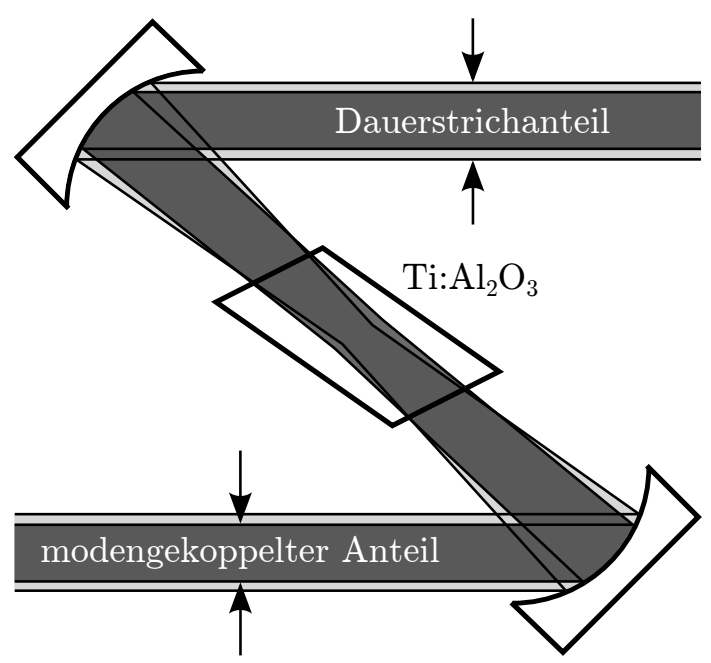

(a)

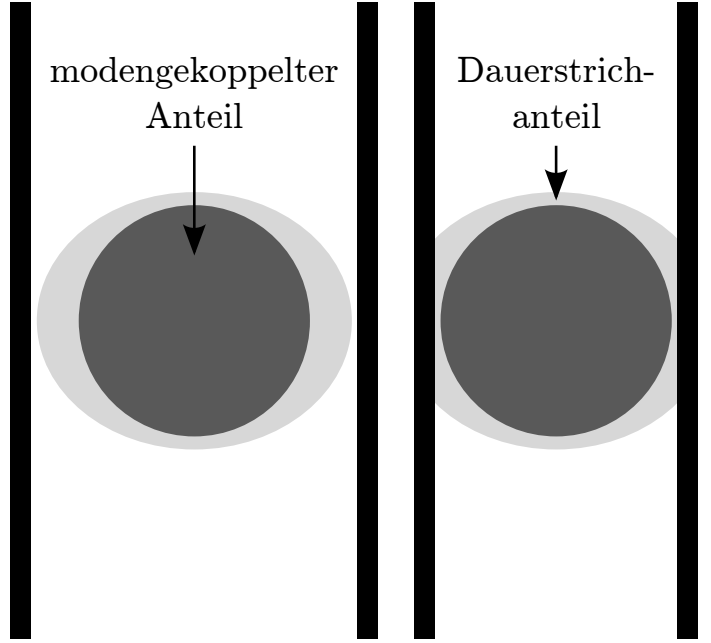

(b)

Abbildung 3.5: (a) Der Saphir fungiert als Kerrlinse und sorgt damit für eine stärkere Fokussierung der modengekoppelten gepulsten Anteile im Strahlengang, verglichen mit dem Dauerstrichanteil. (b) Bei geöffnetem Spalt können sowohl Dauerstrichanteil als auch modengekoppelter Anteil ungehindert die Kavität passieren. Durch Verringerung der Spaltbreite wird der Dauerstrichanteil stark gedämpft.

Durch das breite Emissionsspektrum des $\mathrm{Ti}: \mathrm{Al}_{2} \mathrm{O}_{3}$-Kristalls können sich eine Vielzahl von longitudinalen Moden innerhalb der Kavität ausbilden. Bedingt durch deren Länge von etwa $2 \mathrm{~m}$ unterscheiden sich die benachbarten Moden in ihrer Frequenz um etwa $76 \mathrm{MHz}$, was zudem auch der Frequenz des Pulsbetriebs entspricht. Um die Moden in Phase zu bringen und damit die eigentlichen Pulse zu erzeugen, muss sich eine Kerr-Linse im Saphirkristall ausbilden. Dies jedoch geschieht nur bei ausreichend hoher Intensität, welche durch zufällige Überlagerungen der oft nur wenigen existierenden Moden meist nicht erreicht wird. Aus diesem Grund ist innerhalb der Kavität ein Startelement bestehend aus zwei Quarz-Kristallen verbaut. Diese befinden sich im Strahlengang und können durch schnelle Rotation die effektive Länge der Kavität beeinflussen, was zur simultanen Erzeugung vieler instabiler Moden führt. Aufgrund dessen kann eine genügend hohe Leistungsdichte für die Ausbildung der Kerr-Linse aufgebracht und damit die benötigte Phasenbeziehung zwischen den Moden erzielt werden. Die transversalelektromagnetische Mode entspricht hierbei einer gaußförmigen $\mathrm{TEM}_{00}$-Mode mit einer Beugungsmaßzahl von typischerweise $M^{2}=1.1[270]$. 
Ein Puls, zusammengesetzt aus Moden unterschiedlicher Wellenlänge, unterliegt angesichts der typischerweise nichtlinearen Dispersionsrelation von Feststoffen sowie Flüssigkeiten einer örtlichen Verbreiterung. Bei normaler Dispersion, wie bei den zu durchlaufenden Materialien im Laser der Fall, eilen hierbei die roten Anteile den blauen Anteilen voraus, womit sich eine Gruppengeschwindigkeit von $\mathrm{d} n(\lambda) / \mathrm{d} \lambda<0$ ergibt, mit typischerweise positiver Gruppengeschwindigkeits-Dispersion $\mathrm{d}^{2} n(\lambda) / \mathrm{d} \lambda^{2}$. Des weiteren führt die Selbstphasenmodulation [268] hervorgerufen durch den elektrooptischen Kerr-Effekt [267] infolge unterschiedlicher Intensitäten innerhalb eines Pulses zu einer positiven Gruppengeschwindigkeits-Dispersion. Um beim vielfachen Durchlaufen der Kavität diesen Effekten entgegen zu wirken, wird ein Element mit negativer Gruppengeschwindigkeits-Dispersion benötigt. Realisiert ist dies im Mira durch zwei Quarz-Prismen (P1 und P2 in Abbildung 3.4), bei welchen aufgrund ihrer Positionierung Pulskomponenten mit längerem Wellenanteil eine größere Strecke innerhalb der Prismen zurücklegen. Folglich resultiert daraus eine negative Gruppengeschwindigkeits-Dispersion. Diese sorgt für eine Verkürzung der Pulslänge, was wiederum zu höheren Intensitäten und damit auch steigender Selbstphasenmodulation führt, welche ihrerseits die Pulslänge wieder vergrößert. Die konkurrierenden Prozesse sorgen für eine Soliton-Formation mit sich nicht mehr ändernder Pulsform. Je nach der Position eines Prismas (von außen justierbar) kann dadurch die Pulslänge zwischen etwa 90 - 150 fs variiert werden 270. Infolge dieser Selbstregulierung werden auch kleine Leistungsschwankungen innerhalb der Kavität ausgeglichen und sorgen dadurch für einen energetisch stabilen Betrieb. Bedingt durch die zeitliche Länge der Pulse, welche infolge einer Überlagerung benachbarter Moden zustande kommen, ergibt sich eine spektrale Aufweitung um die zentrale Wellenlänge. Unter der Annahme eines gaußförmigen Pulses liegt das Zeit-Bandbreiten-Produkt bei [271]:

$$
\Delta \nu \cdot \Delta T=\frac{2 \ln (2)}{\pi} \approx 0.44
$$

Somit ergibt sich für eine typische zeitliche Halbwertsbreite von $\Delta T=130 \mathrm{fs}$ eine spektrale Frequenzbandbreite von $\Delta \nu=3.4 \mathrm{THz}$, was unter Annahme eines Modenabstands von $76 \mathrm{MHz}$ etwa 46000 Moden entspricht. Im Fall einer zentralen Wellenlänge von $800 \mathrm{~nm}$ beträgt die spektrale Halbwertsbreite $\Delta \lambda=7.2 \mathrm{~nm}$.

Die Zentralwellenlänge wird durch ein Lyot-Filter [272] ausgewählt. Dieses aus mehreren Quarzscheiben samt Polarisationsfiltern bestehende Filter sorgt unter Ausnutzung der Doppelbrechung, wodurch sich ordentlicher und außerordentlicher Strahl unterschiedlich schnell ausbreiten, für eine Änderung der Polarisation des durchquerenden Lichts. Mit Hilfe der Polarisationsfilter können dadurch nur bestimmte Wellenlängen, bei denen sich dieser Effekt wieder aufhebt das Filter mit einer geringen Dämpfung passieren. Die Auswahl der ungedämpften Wellenlängen kann durch eine Variation der Scheibendicken, hier realisiert durch eine Rotation des Filters senkrecht zum Strahlengang, erfolgen. Ansteuerbar über eine Mikrometerschrau- 
be außerhalb des Laserkopfes kann so der komplette durch den $T i$ : $\mathrm{Al}_{2} \mathrm{O}_{3}$-Kristall verstärkte Bereich angewählt werden (siehe Abbildung 3.7(b)). Die Änderung der Zentralwellenlänge führt durch die nichtlineare Dispersionsrelation zu einer Änderung der Pulslänge, sowie auch der Laufbahn der Pulse innerhalb der Kavität, was durch ein Nachführen des einstellbaren Prismas kompensiert werden kann.

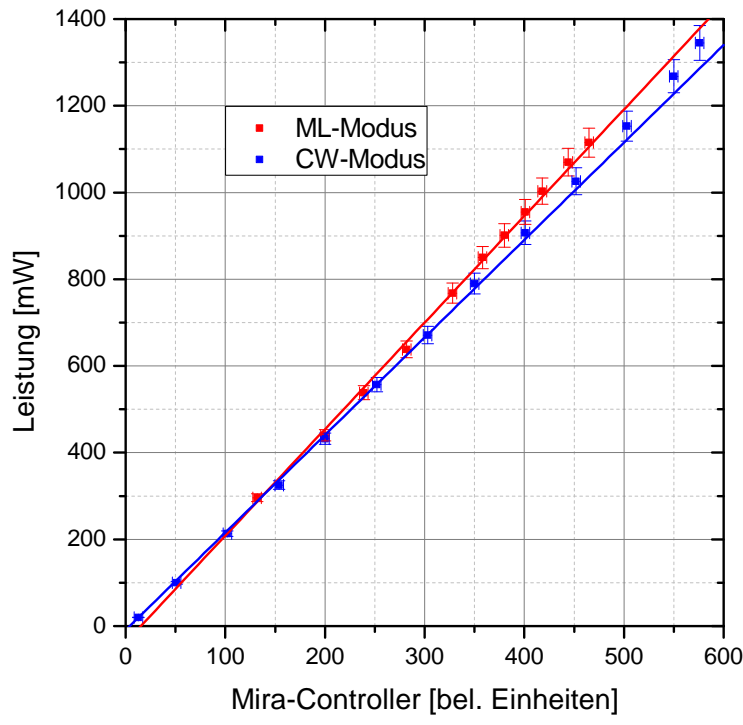

(a)

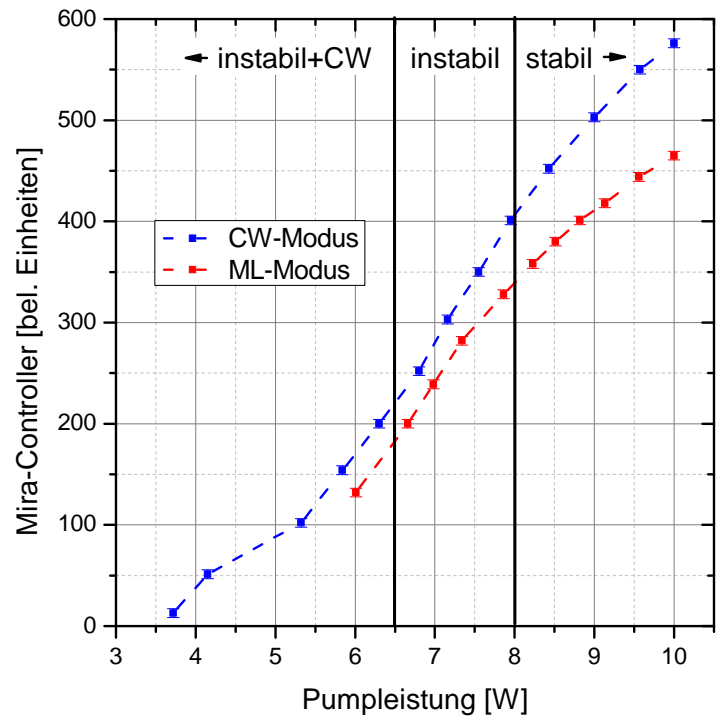

(b)

Abbildung 3.6: (a) Tatsächliche Leistungsabgabe bei einer Wellenlänge von $\lambda_{c}=800 \mathrm{~nm}$ verglichen mit der Anzeige des Mira-Controllers in den verschiedenen Betriebsmodi. Die Geraden stellen die jeweilige lineare Anpassung an die Messdaten dar, wobei nicht von strenger Proportionalität ausgegangen wird. (b) Abhängigkeit der Messanzeige von der zugeführten Pumpleistung. Oberhalb von $8 \mathrm{~W}$ zeigt sich ein stabiles Pulsverhalten, während unterhalb von $6.5 \mathrm{~W}$ ein starker Anstieg der Dauerstrichleistung zu beobachten ist.

Die elektronische Ansteuerung des Laserkopfes erfolgt über den zugehörigen Controller, welcher auch die Regelung im Falle einer installierten Pikosekundeneinheit übernimmt. Im Femtosekunden-Betrieb besteht die einzige zu steuernde aktive Komponente, ausgenommen sei hier die Versorgung des Antriebs eines Etalon, aus dem Starter, welcher zur Initialisierung im Pulsbetrieb in Bewegung gesetzt wird. Ferner überwacht der Controller mittels Photodetektoren die durchschnittliche Laserleistung und, unter Zuhilfenahme des Etalon, den Dauerstrichanteil im Strahlengang. Letzterer registriert einen durchschnittlichen Leistungsanteil von weniger als 1\%, was einem Aspektverhältnis zwischen diesem und der Spitzenleistung im Pulsbetrieb von $1 \times 10^{9}$ entspricht [270]. Zusätzlich liefert der Controller ein mittels einer schnellen Photodiode mit dem Laser synchronisiertes gepulstes Signal. Zur Bestimmung der 
Luftfeuchtigkeit, interessant für den Betrieb zwischen $920 \mathrm{~nm}$ und $980 \mathrm{~nm}$, befindet sich zudem ein Hygrometer im Laserkopf. Im genannten Wellenlängenbereich als auch bei $760 \mathrm{~nm}, 820 \mathrm{~nm}$ und $900 \mathrm{~nm}$ befinden sich starke Wasser-Absorptionslinien, welche einen Pulsbetrieb in den entsprechenden Energiebändern bei Raumluft verhindern 270,273. Abhilfe schafft hier eine Flutung des Laserkopfes mit Stickstoffgas. Bei einer Absenkung der Luftfeuchte auf einen Wert unter $10 \%$ ist auch bei diesen Energien ein stabiles Pulsen möglich. Eine einheitenlose numerische Anzeige am Controller gibt Auskunft über die momentane, sowohl vom Dauerstrich- als auch vom gepulsten Anteil stammende, den Laserkopf verlassende Strahlintensität. Verglichen mit der tatsächlichen abgegeben Leistung verhält sich die Anzeige bezüglich einer zentralen Wellenlänge nahezu linear, wenn auch unterschiedlich für die verschiedenen Betriebsmodi (siehe Abbildung 3.6(a)). Mit ansteigender Wellenlänge nimmt deren Sensitivität von $700-1000$ nm etwa um einen Faktor von 2.5 ab (siehe Abbildung $3.7(b))$. Auch die Leistungsabgabe selbst zeigt ein stark wellenlängenabhängiges Verhalten mit einem Leistungsmaximum um $800 \mathrm{~nm}$ (siehe Abbildung 3.7(a)). Bei gleicher Pumpleistung liegt ein Verhältnis von etwa vier zwischen $800 \mathrm{~nm}$ und dem niedrigsten noch pulsbaren Bereich (1000 nm) vor. Außerhalb des 700-1000 nmIntervalls ist kein stabiler Pulsbetrieb möglich. Pumpleistungsabhängige Analysen bei justierter Kavität zeigen oberhalb einer Pumpleistung von $3.5 \mathrm{~W}$ einen messbaren Laser-Output im Dauerstrichbetrieb (siehe Abbildung 3.6(b)). Unterhalb von 6.5 W kann im Pulsbetrieb das Dauerstrichsignal nicht vollständig eliminiert werden. Oberhalb ist ein reiner Pulsbetrieb mit ggf. kurzen Unterbrechungen und Instabilitäten möglich. Ab $8 \mathrm{~W}$ Pumpleistung stellt sich ein stabiler Puls ohne weitere Störungen ein. Die absoluten Leistungsangaben hängen stark von der momentanen Justage, wie auch dem Grad der Verunreinigungen, hauptsächlich hervorgerufen durch Staub, innerhalb der Laserkavität ab.

\section{Justage}

Bei intakter Grundkonfiguration innerhalb der Kavität muss sich für den Betrieb um $800 \mathrm{~nm}$ lediglich das bewegliche Prisma innerhalb des Strahlenganges befinden und der von außen rotierbare Endspiegel M7 (siehe Abbildung 3.4) um seine Horizontale gedreht werden, bis ein Lasersignal registriert wird. Anschließend kann die Leistung weiter optimiert werden. Zur Erzielung einer zentrierten lateralen Spaltposition kann dieser iterativ durch Verkleinerung, einem damit verbundenen Einbruch der Leistung, und anschließender seitlicher Bewegung bis zur Leistungsmaximierung auf eine exakte zum Strahl zentrale Position gebracht werden. Ein zu kleiner Spaltdurchmesser führt zu einer periodischen Schwankung der Pulsleistung, welche über ein Oszilloskop beobachtet werden kann. Der Dauerstrichanteil verschwindet im gepulsten Betrieb typischerweise abrupt und reduziert sich daher bei richtiger Einstellung auf eine nicht mehr messbare Intensität. Im Energiebereich starker Wasserabsorptionen springt der Laser nicht zum Pulsbetrieb an, weshalb die Wellenlänge schrittweise während eines 


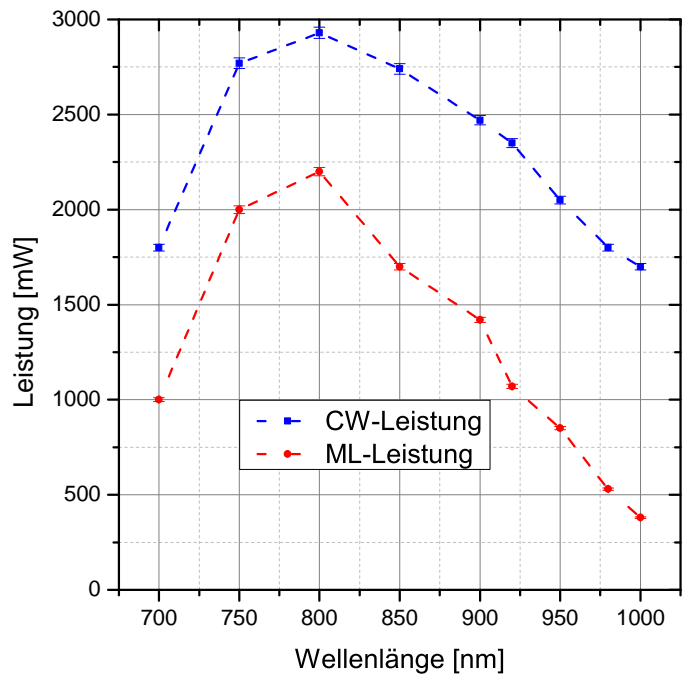

(a)

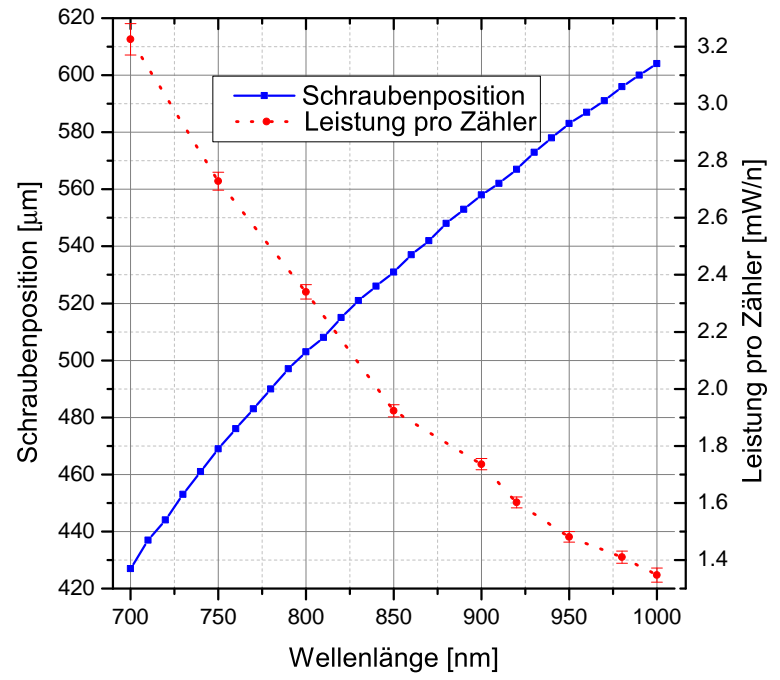

(b)

Abbildung 3.7: (a) Wellenlängenabhängige Maximalleistung bei einer Pumpleistung von $10 \mathrm{~W}$ im Dauerstrich- sowie im Pulsmodus. Außerhalb des dargestellten Bereichs ist kein Pulsbetrieb möglich. (b) Position der Mikrometerschraube zur Einstellung des doppelbrechenden Lyot-Filters zur Auswahl der gewünschten Wellenlänge (von Coherent vermessen). Rot dargestellt ist die absolute Sensitivität der Anzeige des Mira-Controllers auf die verschiedenen Wellenlängen. Die Geraden zwischen den Messpunkten innerhalb beider Darstellungen dienen lediglich der Orientierung.

stabilen modengekoppelten Betriebs an die Zielposition angepasst werden muss. Hierbei müssen das bewegliche Prisma mit dem Strahl sowie der Endspiegel M7 horizontal nachgeführt werden. Die Komponenten innerhalb der Kavität erwärmen sich, wie auch den am Saphir sowie Pumplaser angeschlossenen Wasser-Kühlkreislauf zu Beginn der Inbetriebnahme, was größere Leistungsschwankungen innerhalb der ersten 30 min zur Folge hat.

\subsubsection{Pulspicker}

Zur Selektion einzelner Pulse für Experimente mit Zeitfenstern von bis zu $100 \mu \mathrm{s}$ Länge kommt der Pulspicker Model 9200 (Coherent, Inc) zum Einsatz. Hauptbestandteil ist ein akustooptischer Modulator [274], auch Bragg-Zelle genannt, zur Umlenkung einzelner Femtosekunden-Pulse. Als akustooptisches Material findet hierbei $\mathrm{SiO}_{2}$ Verwendung. Über einen piezoelektrischen Wandler, bestehend aus $\mathrm{LiNbO}_{3}$, werden hochfrequente akustische Wellen $(380 \mathrm{MHz})$ in den Quarz induziert 275]. Diese rufen Dichteschwankungen und damit periodische Änderungen des 
Brechungsindexes innerhalb des Materials hervor [276]. Das daraus resultierende periodische Gitter, welches als longitudionale ebene Druckwelle aufgefasst werden kann, führt zu einer Beugung des einfallenden Laserpulses (siehe Abbildung 3.8(a)). Im Falle des Bragg-Regimes findet die Beugung hauptsächlich in der ersten Ordnung mit einem Maximum an Intensität bei Lichteinfall im Bragg-Winkel von 274:

$$
\Theta_{B} \approx \frac{\lambda}{2 n \Lambda}
$$

statt. Mit einer optischen Wellenlänge $\lambda=800 \mathrm{~nm}$, Brechungsindex $n=1.46$ [276] und einer akustischen Wellenlänge der ebenen Welle im Quarz von $\Lambda=15.7 \mu \mathrm{m}$ wird eine optimale Beugung bei einem Winkel von $\Theta_{B}=17.5 \mathrm{mrad}$ (etwa $1.00^{\circ}$ ) erreicht. Da nur einzelne Pulse ausgewählt werden sollen, ist die Modulationsdauer durch die Laserfrequenz des Mira von $76 \mathrm{MHz}$ auf eine Zeit von maximal 13.2 ns begrenzt. Mit einer Anregung über eine Periode von $10 \mathrm{~ns}$ bei einer Schallgeschwindigkeit von $5.96(3) \mathrm{km} / \mathrm{s}$ für longitudionale Wellen in Quarz [277] ergibt sich somit ein Wellenpaket einer Länge von etwa $60 \mu \mathrm{m}$. Um lediglich einen Puls umzulenken wird der einfallende Laser mittels eines gewölbten Spiegels (10 cm Radius) auf die BraggZelle fokussiert. Die resultierende Fokusgröße liegt jedoch über der des Wellenpaketes, da kleinere Strahldurchmesser zur Beschädigung des Quarz führen können. Die Leistung eines abgelenkten Pulses wird daher auf etwa $50 \%$ reduziert [275]. Um die Ausbildung stehender Wellen durch Reflexionen an den Enden der Bragg-Zelle $\mathrm{zu}$ verhindern, die unbeabsichtigt weitere Pulse beugen könnten, ist eine Seite des Quarz schräg gegenüber der Grundfläche abgeschliffen und aufgeraut. Nach räumlicher Auftrennung der beiden Komponenten wird der unbeeinflusste Teil über eine Strahlfalle geblockt und nur der abgelenkte Puls kann den Pulspicker passieren (siehe Abbildung 3.8(b)).

Eingestellt wird der Pulspicker über einen zugehörigen Controller, welcher die elektrische Ansteuerung der Bragg-Zelle übernimmt. Variiert werden können zeitliche Länge, Amplitude, Phase und Verzögerung der Anregung mittels Hochfrequenzverstärker in Synchronisation mit der schnellen Photodiode im Mira. Exemplarisch ist die Ausgangsspannung für ein Wellenpaket in Abbildung 3.9 (b) dargestellt. Das Teilungsverhältnis kann in einem Bereich zwischen 16 und 8096 gewählt werden, was Pulsperioden von $211 \mathrm{~ns}$ bis hin zu $107 \mu$ s entspricht. Bei korrekt justierten Parametern, welche für jeden Repititionsbereich neu gefunden werden müssen, sind Intensitätsverhältnisse zwischen ausgewählten und adjazenten Pulsen von über 100 : 1 möglich. Zu zeitlich weiter entfernten Pulsen sind Verhältnisse größer als 1000: $1 \mathrm{zu}$ erreichen 275]. In Abbildung 3.10(a) ist das resultierende Laser-Signal hinter dem Pulspicker nach optimaler Justage dargestellt. Falsche Verzögerungszeiten sowie zu lange Anregungsdauern führen zur Beugung teils mehrerer Pulse (siehe Abbildung 3.10(b)). Insbesondere die Einstellung der Strahlfalle hat starken Einfluss auf den Signaluntergrund wie auch auf die resultierende Leistung. Bei zu niedrig positionierter Falle können auch nicht gebeugte Anteile den Pulspicker, wie in 


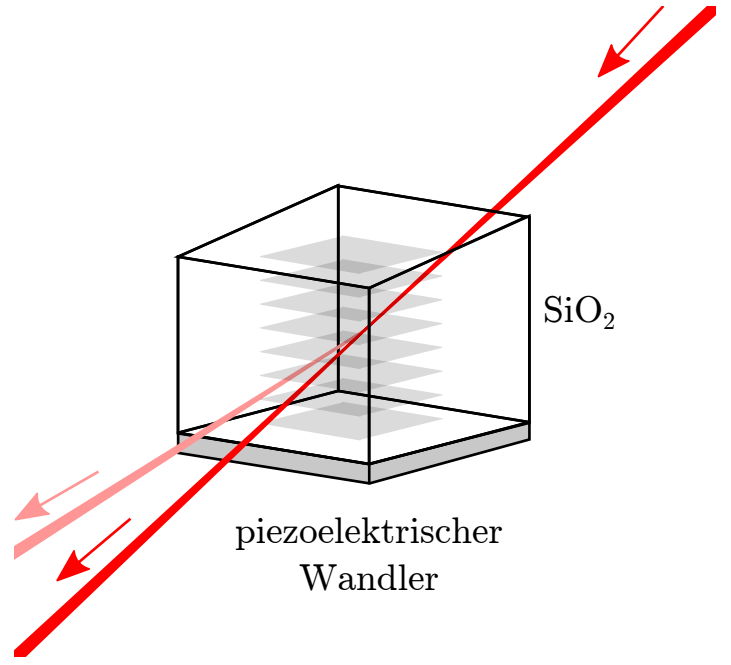

(a)

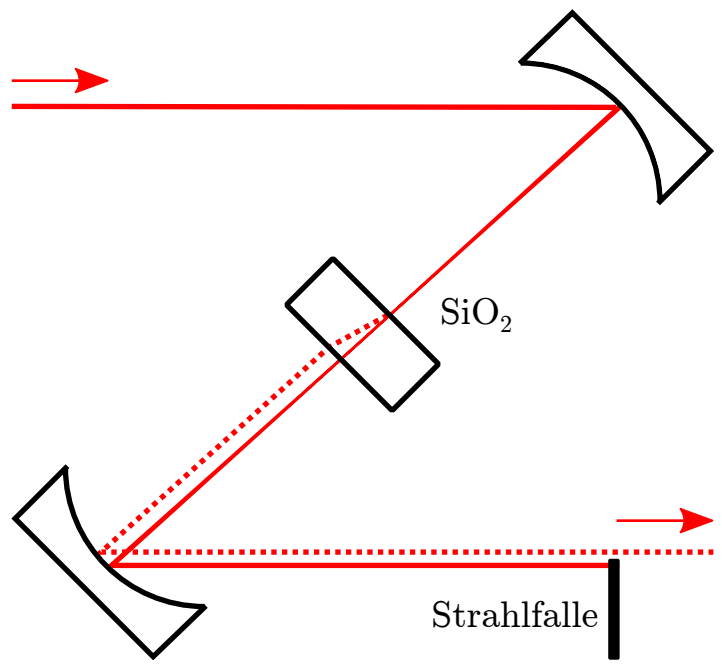

(b)

Abbildung 3.8: (a) Der sich unter dem Quarz befindliche piezoelektrische Wandler induziert akustische Wellenzüge, die zur Beugung eines eintreffenden Laserpulses führen. Durch einen schiefen Anschliff des Quarzes können sich keine stehenden Wellen und damit ungewollte Interferenzen ausbilden. (b) Für ein präzises Auswählen lediglich eines Pulses wird der einfallende Laser über einen Hohlspiegel auf den Quarz fokussiert. Der unbeeinflusste Anteil kann durch eine Strahlfalle innerhalb des Pulspickers absorbiert werden.

Abbildung 3.9 (a) dargestellt, verlassen. Für die experimentelle Nutzung wird in den in dieser Arbeit vorgestellten Messungen mit Pulspicker jederzeit die höchstmögliche Stellposition der Strahlfalle verwendet, da dies den Untergrund auf ein bestmögliches Minimum reduziert.

\subsubsection{Harmonischer Generator}

Für die Messung von Lumineszenzsignalen im sichtbaren sowie UV-Bereich wird in den hier untersuchten Materialsystemen auch entsprechend kurzwelliges Licht zur Anregung benötigt. Dies ist durch Frequenzmischung der Pulse des Mira und einer einhergehenden Erzeugung höherer harmonischer Anteile realisiert. Hierfür werden die nichtlinearen Polarisationseigenschaften von verschiedenen Kristallen ausgenutzt 278]. Erstmals beobachtet wurde dieser Effekt von Franken et al. 1961 mit Hilfe eines gepulsten Rubinlasers und einem Quarzkristall [279]. Zur effizienten Erzeugung der zweiten Harmonischen (SHG) müssen die nichtlinearen dielektrischen Koeffizienten für die Polarisation zweiter Ordnung groß als auch der Kristall transparent für Fundamentale und Harmonische sein. Im Allgemeinen besteht die Polarisationsmatrix zweiter Ordnung, analog zur piezoelektrischen, aus 18 unabhängigen 


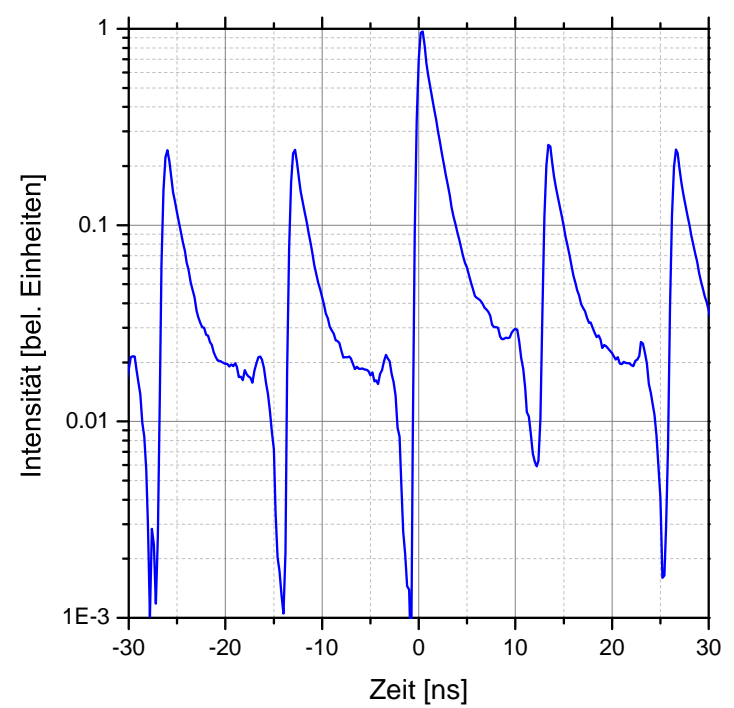

(a)

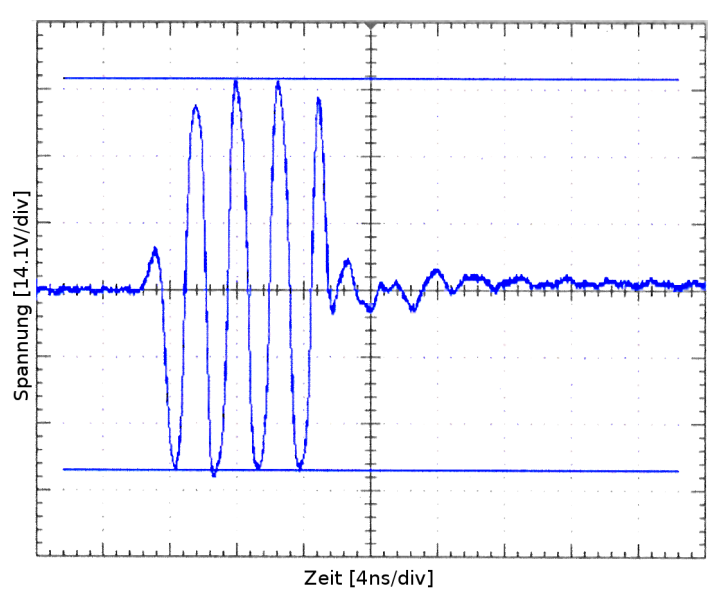

(b)

Abbildung 3.9: (a) Durch eine zu niedrig eingestellte Strahlfalle kann auch ein großer Teil des nicht abgelenkten Signals den Pulspicker passieren. Die starken Einbrüche hinter den abfallenden Flanken sind messtechnischer Natur. (b) Ausgangsspannung des Verstärkers bei voller Leistung zum Umlenken eines Laserpulses (von Coherent vermessen). Die Spannungsdifferenz zwischen den beiden blauen Linien beträgt $82.6 \mathrm{~V}$. Eine Hauptunterteilung der X-Achse entspricht 4 ns.

Komponenten, welche multipliziert mit dem sechs-komponentigen quadratischen Spaltenvektor $\boldsymbol{E} \boldsymbol{E}$ der elektrischen Feldstärke die Polarisation $P^{(2)}$ ergeben [278]. Im einfachsten Fall einer monochromatischen ebenen Welle der Frequenz $\nu$ und nur einer aktiven Komponente $d$ innerhalb der Polarisationsmatrix ergibt dies (nach [268]):

$$
\begin{aligned}
P^{(2)} & =\varepsilon_{0} d E^{2} \\
& =\varepsilon_{0} d\left[E_{0} \cos (2 \pi \nu t)\right]^{2} \\
& =\frac{1}{2} \varepsilon_{0} d E_{0}^{2}+\frac{1}{2} \varepsilon_{0} d E_{0}^{2} \cos (4 \pi \nu t) \\
& =P^{(2)}(0)+P^{(2)}(2 \nu) .
\end{aligned}
$$

Der erste Term beschreibt hierbei eine Ladungsseparation im Material, was zu einer zusätzlichen konstanten Polarisation führt, während der zweite eine Welle mit doppelter Frequenz zur fundamentalen darstellt. Die Kopplungen $d$ sind betragsmäßig sehr klein $\left(\approx 10^{-13}-10^{-12} \mathrm{~m} \mathrm{~V}^{-1}[279]\right)$, wodurch hohe elektrische Feldstärken zur Erzeugung einer SHG benötigt werden. Des weiteren hängt die Effizienz von der Phase der Harmonischen zur Fundamentalen im Interaktionsbereich ab. Bei unterschiedlichen Gruppengeschwindigkeiten entlang der Strecke aufgrund der verschiedenen Wellenlängen und nichtlinearer Dispersionsrelation führt dies 


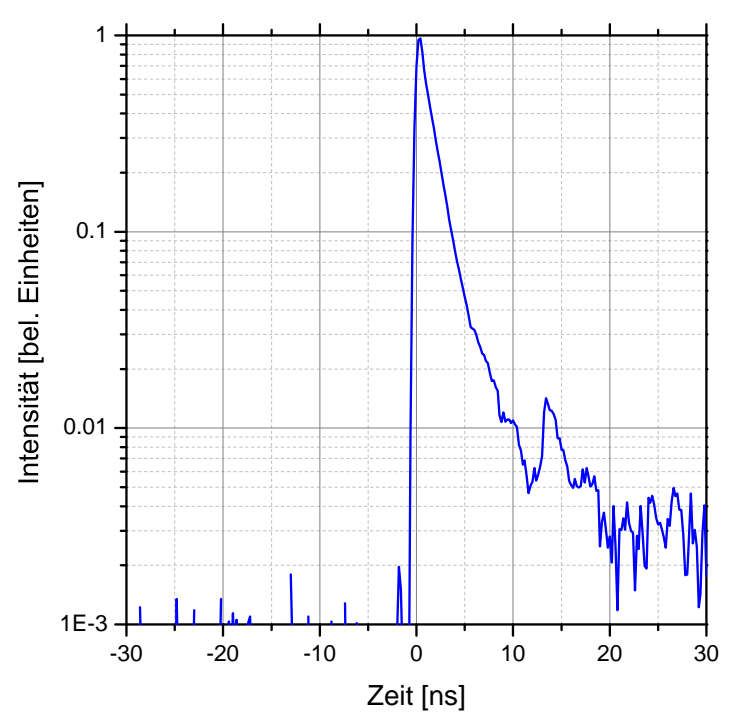

(a)

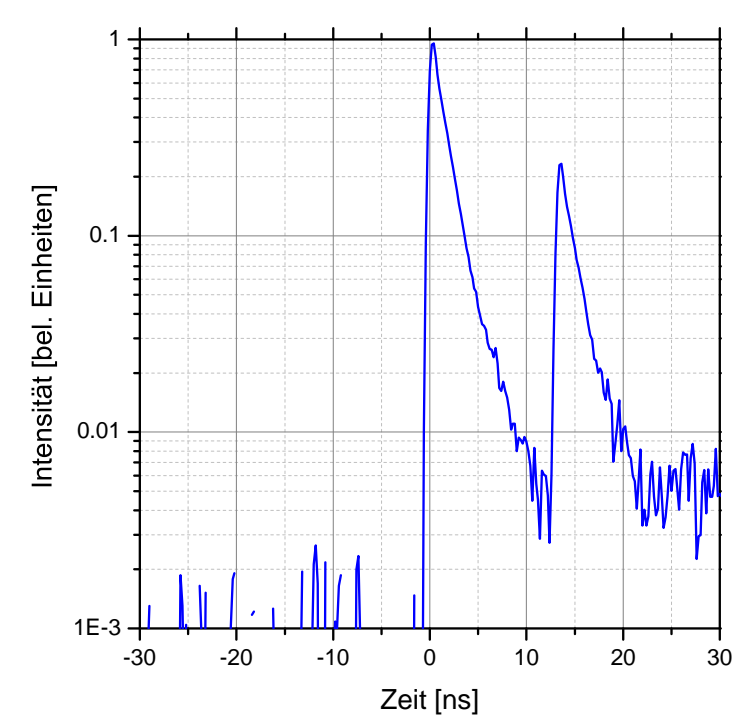

(b)

Abbildung 3.10: (a) Laserintensität nach dem korrekt eingestellten Pulspicker. Der adjazente Puls bei 13.2 ns zeigt eine Abschwächung gegenüber dem ausgewählten primären Puls auf etwa 1\%. Die lange abfallende Flanke am Hauptpuls ist der Geschwindigkeit der genutzten Photodiode geschuldet. (b) Bei phasenverschobenem Betrieb zum Laser kann eine Umleitung von zwei Pulsen erfolgen, was in Experimenten dann auch zu Doppelanregungen führt.

zu destruktiven Interferenzen und einem damit einhergehenden Intensitätsverlust. Unter Ausnutzung der Doppelbrechung innerhalb des Kristalls kann durch entsprechende Orientierung (typischerweise durch Rotation) des selbigen dieser Effekt ausgeglichen werden. Für unterschiedliche Wellenlängen sind somit aber auch jeweilige unterschiedliche Orientierungen notwendig. Bei der Erzeugung dritter (THG) als auch vierter Harmonischer (FHG) direkt aus der Fundamentalen handelt es sich um Prozesse dritter bzw. vierter Ordnung, welche verglichen zur zweiten Ordnung noch einmal deutlich geringere Effizienzen aufweisen. Um solch niedrige Wirkungsgrade zu vermeiden, können THG und FHG auch über mehrstufige Prozesse durch Mischung der Fundamentalen mit der SHG bzw. einer Mischung der SHG mit sich selbst gebildet werden.

Eine schematische Zeichnung des in diesem Aufbau verwendeten harmonischen Generators HarmoniXX (A.P.E) ist in Abbildung 3.11 dargestellt. Die nichtlinearen Kristalle X1 - X3 können je nach Konfiguration aus ihren entsprechenden Halterungen herausgenommen werden. Nach dem ersten Kristall zur Erzeugung der SHG befindet sich ein linearer Kristall (C1) mit nahezu identischem Brechungsindex aber entgegengesetzter Neigung verglichen zum nichtlinearen Kristall X1. Dies dient einer Kompensation des Strahlversatzes zwischen Fundamentaler und SHG. Bevor die 
beiden Strahlenanteile auf X2 treffen, wird eine Laufzeitanpassung mittels zweier Prismen, vergleichbar zur Anordnung im Mira (siehe Kapitel 3.1.2), durchgeführt (DC1). Des weiteren wird die Polarisationsrichtung zwischen Fundamentaler und SHG, welche zuvor orthogonal zueinander stehen, durch Rotation der verarmten Fundamentalen von horizontaler in die vertikale Ebene mit Hilfe einer Wellenplatte 280] (WP1) zusammengebracht. In X2 bildet sich bei angepasster Justage nun die dritte Harmonische. Alternativ zu X2 kann an diese Position noch ein anderer Kristall zur Erzeugung einer FHG 2+2 aus der Mischung der SHG mit sich selbst gewonnen werden. Die FHG 2+2 arbeitet jedoch nur im Bereich zwischen 840 -920 nm und deckt somit einen Wellenlängenbereich von 210-230 nm ab 281]. Zur vorzeitigen Auskopplung verschiedener Harmonischer dienen Seperatoren (S1 - S4), bestehend aus zwei planparallelen Spiegeln, welche für andere Strahlenteile transparent und damit durchlässig erscheinen. Aufgrund der weiten Durchstimmbarkeit des Systems ist die Auskopplung der THG über zwei verschiedene Seperatoren für den kurzwelligen bzw. langwelligen Bereich realisiert. Für die Erzeugung von Wellenlängen unterhalb der $210 \mathrm{~nm}$ werden THG und Fundamentale analog zur vorherigen Methode in die gleiche Polarisationsebene gebracht und Laufzeitunterschiede ausgeglichen. Als Verzögerungskompensator wird hier jedoch eine Aufspaltung des Strahlenganges (S3 rechts) zwischen Fundamentaler und THG gewählt. Dies ermöglicht neben dem Ausgleich der Laufzeiten auch eine Repositionierung der Fundamentalen bezüglich der THG um einen möglichst genauen Überlapp beider Strahlanteile zu erzielen. Nach abschließender Fokussierung über die Linse L3 auf den Kristall X3 kann somit die FHG 3+1 innerhalb des Wellenlängenbereichs von 190 - $210 \mathrm{~nm}$ 281] erzeugt werden. Die Fokussierung auf die Kristalle X1 und X2 kann bei geschlossenem Gehäuse über die Drehknöpfe K1 und K2 vorgenommen werden. Die blau dargestellten Drehknöpfe K3 - K5 dienen der Einstellung des Rotationswinkels der Kristalle, wobei K5 erst nachträglich an das Gehäuse angebracht wurde.

In Abhängigkeit zur Pumpleistung zeigen SHG und THG im stabilen Pulsbereich einen nahezu linearen Verlauf (siehe Abbildung 3.12(a)). Direkt nach der Installation konnten bei einer Pumpleistung von $1.88 \mathrm{~W}$ (bei $\lambda=800 \mathrm{~nm}$ ) für die SHG maximale Leistungsspitzen von $628 \mathrm{~mW}$ erzielt werden. Über die Zeit zeigt der Leistungsverlauf der Harmonischen ein konstantes Verhalten, wobei kurzzeitige Fluktuationen mit Schwankungen innerhalb eines Bereichs von $1 \%$ auftreten (siehe Abbildung 3.12 (b)). Die Leistung der FHG 3+1 für verschiedene Konfigurationen ist in Tabelle 3.1 dargestellt. Die FHG wurde hierfür nach dem Seperator S4 vermessen. Insbesondere nach diesem Seperator zeigt sich jedoch ein relativ starker Untergrund bestehend aus den verarmten Signalen der Fundamentalen, SHG und THG. Eine saubere Trennung kann mit Hilfe eines Prismas erreicht werden, wodurch sich jedoch die Leistung der FHG ebenfalls stark mindert. 


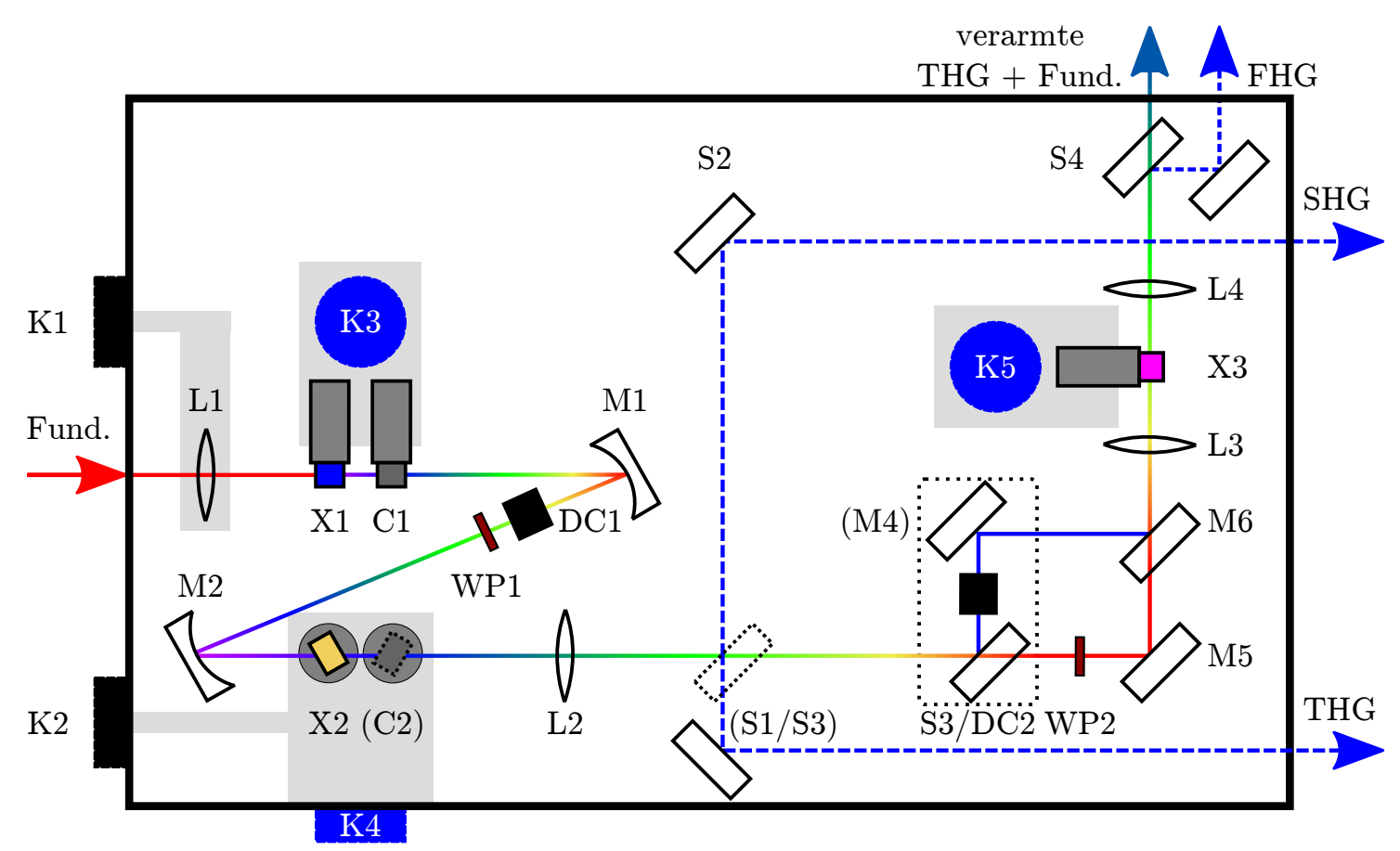

Abbildung 3.11: Schematische Darstellung des HarmoniXX Generators in der Konfiguration zur Erzeugung der FHG 3+1. Die Fundamentale fällt über die Linse L1 auf den Kristall X1, wobei die zweite Harmonische generiert wird. Im Kristall X2 wird aus Fundamentaler und zweiter Harmoischer die dritte Harmonische und zusammen mit X3 letztendlich die vierte Harmonische FHG 3+1 erzeugt. Die weiteren Elemente im Strahlengang dienen wie im Text beschrieben der Phasensowie Gruppengeschwindigkeitsanpassung der unterschiedlichen Strahlanteile. Zur vorzeitigen Auskopplung von SHG bzw. THG können optional die Separatoren S1/S2 eingebracht werden. Bei korrekter Vorjustage kann die Ausgangsleistung bei geschlossenem Gehäuse mit Hilfe der Drehknöpfe K1-K5 optimiert werden. 


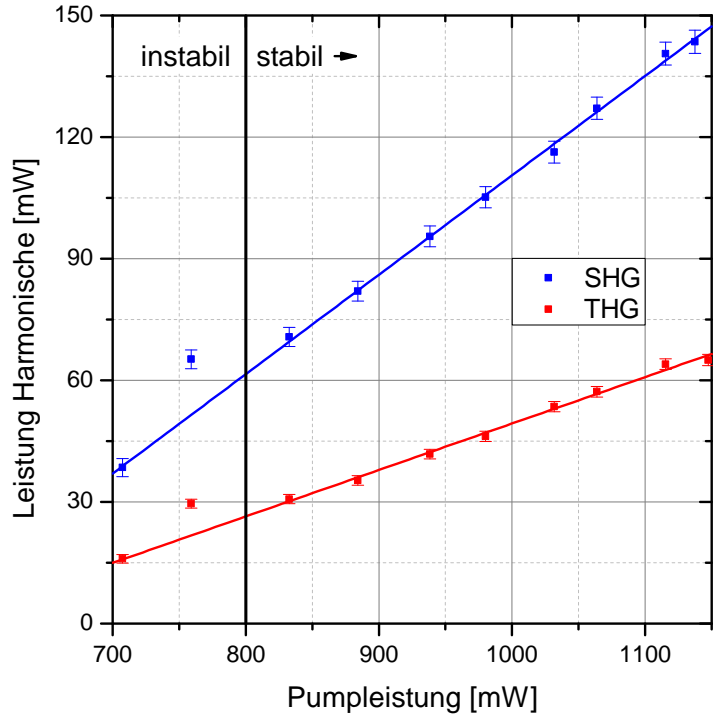

(a)

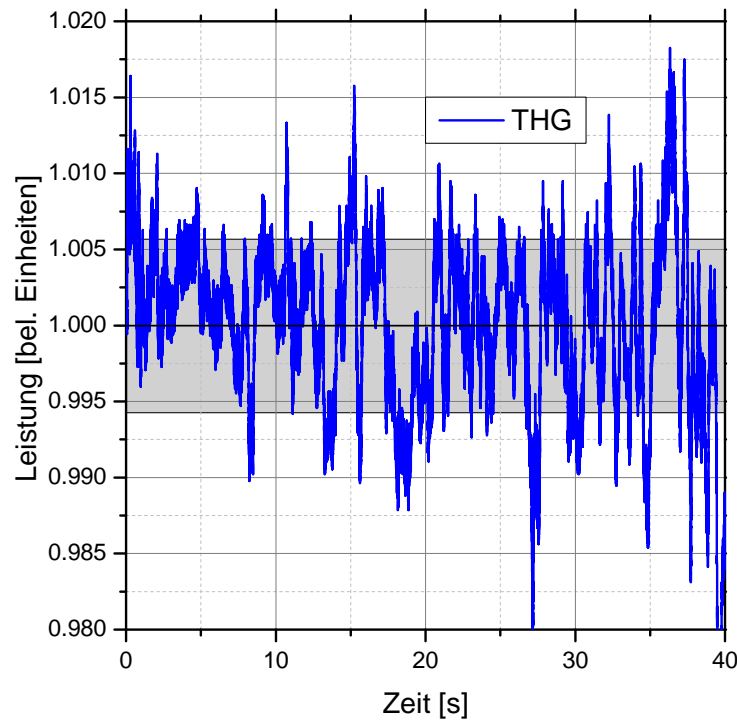

(b)

Abbildung 3.12: (a) Abhängigkeit der zweiten und dritten Harmonischen von der einfallenden Pumpleistung im Pulsbetrieb bei einer Anregungswellenlänge von $\lambda=$ $800 \mathrm{~nm}$. Im instabilen Regime tritt zunächst eine Sättigung auf, welche in der linearen Anpassungen nicht berücksichtigt wurde. Die Fehler rühren hauptsächlich von der Pumpleistung, berechnet aus Controller-Werten, her. (b) Zeitliche Schwankung der THG-Leistung. Die Standardabweichung (grau unterlegt) beträgt $0.58 \%$.

\begin{tabular}{ccc}
\hline Konfiguration & $\begin{array}{c}\text { Abstand zu Mira } \\
{[\mathbf{c m}]}\end{array}$ & $\begin{array}{c}\text { FHG 3+1 Leistung } \\
{[\mathbf{m W}]}\end{array}$ \\
\hline keine Linse & - & 0.2 \\
2000 mm Linse & 47 & 0.4 \\
2000 mm Linse & 77 & 0.8 \\
1000 mm Linse & 49 & 1.1 \\
1000 mm Linse & 63 & 1.3 \\
1000 mm Linse & 67 & 1.4 \\
1000 mm Linse & 75 & 0.6 \\
PP 2 W \& 2000 mm Linse & hinter PP & 2 \\
PP 2 W & - & $1.9-2.1$ \\
\hline
\end{tabular}

Tabelle 3.1: Abhängigkeit der Leistung der FHG für verschiedene Konfigurationen. Ohne Pulspicker (PP) verbessert eine $1000 \mathrm{~mm}$ Linse die Leistung signifikant. Der Pulspicker selbst sorgt für eine leichte Fokussierung, wodurch im Falle hoher Pumpleistungen vom Mira (hier $2 \mathrm{~W}$ ) ebenfalls hohe FHG-Leistungen erzielt werden können. 


\subsubsection{Chopperrad}

Zur Messung von zeitabhängigen Lumineszenzspektren mit Transienten größer als $100 \mu$ s kommt statt des Pulspickers (siehe Kapitel 3.1.3) ein Chopperrad (SR540, Stanford Research Systems, Inc.) zum Einsatz. Der Pulspicker ist zum einen aufgrund seines Teilungslimits auf Perioden von $107 \mu$ s limitiert, zum anderen würde die Messzeit bedingt durch die niedrige Durchschnittsleistung für lange Zeitfenster stark ansteigen (vgl. [282]). Mit dem vorhandenen Chopperrad mit sechs durchlässigen Fenstern lassen sich Teilungen des Lasersignals von $4-400 \mathrm{~Hz}$ realisieren 283]. Signifikant für die resultierende Zeitauflösung sind die Anstiegs- bzw. Abfallzeiten, sowie die Stabilität der Frequenz über den Messzeitraum. Zur Minimierung der Flankendauer wird das Lasersignal über einen Nulldurchgang, realisiert mit Hilfe von zwei Linsen, auf das Chopperrad fokussiert und danach wieder parallelisiert. Mit einer Periodendauer von $T=3.33(2) \mathrm{ms}$ (Chopperfrequenz $f_{c}=300(1) \mathrm{Hz}$ ) ergeben sich so Flankenzeiten von etwa 3.9(2) $\mu$ s. Die Zeitkonstante wird hierbei aus der Zeit der Signalpegeländerung von $10 \%$ zu $90 \%$ (abfallend von $90 \%$ zu $10 \%$ ) des Maximalwertes ermittelt. In Abbildung 3.13 sind exemplarisch die Flankenzeiten für 300 Perioden aufgetragen. Während die aufsteigenden Flanken relativ kompakt verteilt um $3.9 \mu$ s auftreten, zeigen sich bei den abfallenden Teilen mehrere unterschiedliche getrennte Bereiche. Dieser Effekt wird jedoch hauptsächlich durch die Abtastung des genutzten Oszilloskops hervorgerufen. Da sich der Laser stets im gepulsten Betrieb befindet, ergibt sich eine Unterabtastung des eigentlichen Signals einhergehend mit Aliasing [284] einer scheinbar modulierten Amplitude.

Größeren Einfluss auf die zu erzielende Zeitauflösung verübt jedoch die zeitliche Fluktuation der Flanken und Perioden untereinander. Dieses als Jitter bezeichnete Phänomen beschreibt die kurzzeitige zeitliche Variation von digitalen Ereignissen bezogen auf ihre in der Zeit ideale Position [285]. Kurzzeitig bezieht sich hier typischerweise auf Frequenzen oberhalb von $10 \mathrm{~Hz}$ [285, 286]. In Abbildung 3.14 ist der Ausschnitt eines Augendiagramms der gemessenen Flanken dargestellt. Hierfür wurden die einzelnen Perioden in Bezug zu einem jeweiligen Referenzpunkt miteinander überlagert. Die Referenz gibt hierbei ein vom Controller des Chopperrads ausgegebenes TTL-Signal, welches auch bei den Messungen als Trigger Anwendung findet. $\mathrm{Zu}$ erkennen ist eine deutlich breitere Verteilung der Signale verglichen mit den eigentlichen Flankenzeiten.

Jitter lässt sich grundsätzlich in zwei Kategorien aufteilen: zufälliger Jitter (RJ) und deterministischer Jitter (DJ) [287]. Bei zufälligem Jitter handelt es sich um zeitliches Rauschen, welches sich nicht durch erkennbare Abfolgen beschreiben lässt. Typischerweise wird dabei, begründet auf dem zentralen Grenzwertsatz [288], eine Normalverteilung der Ereignisse angenommen. Die Normalverteilung, da im Träger nicht beschränkt, kann somit bei beliebig vielen Ereignissen zu beliebig großen, wenn auch entsprechend seltenen, Jitterabweichungen führen. Im Gegensatz hierzu ist deterministischer Jitter an sich vorhersagbar und in seiner Amplitude 


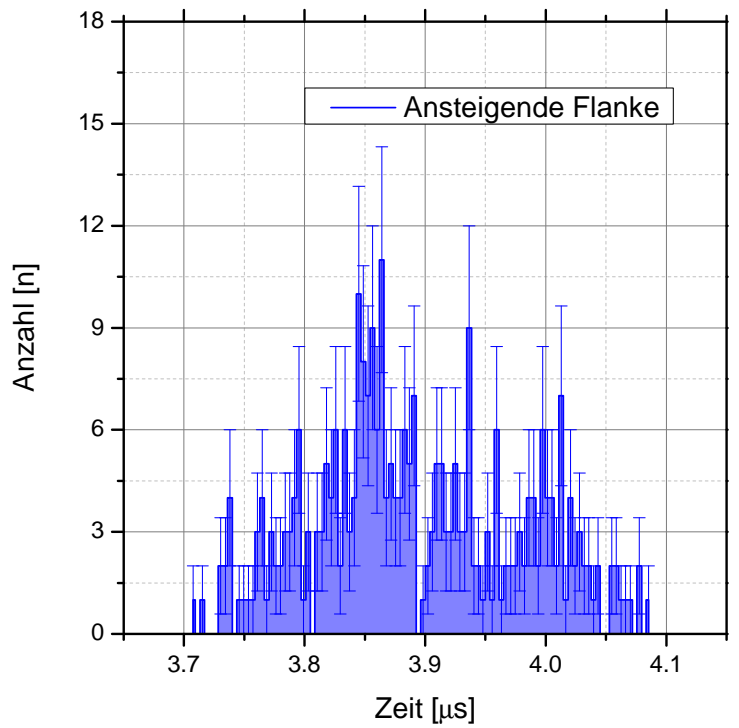

(a)

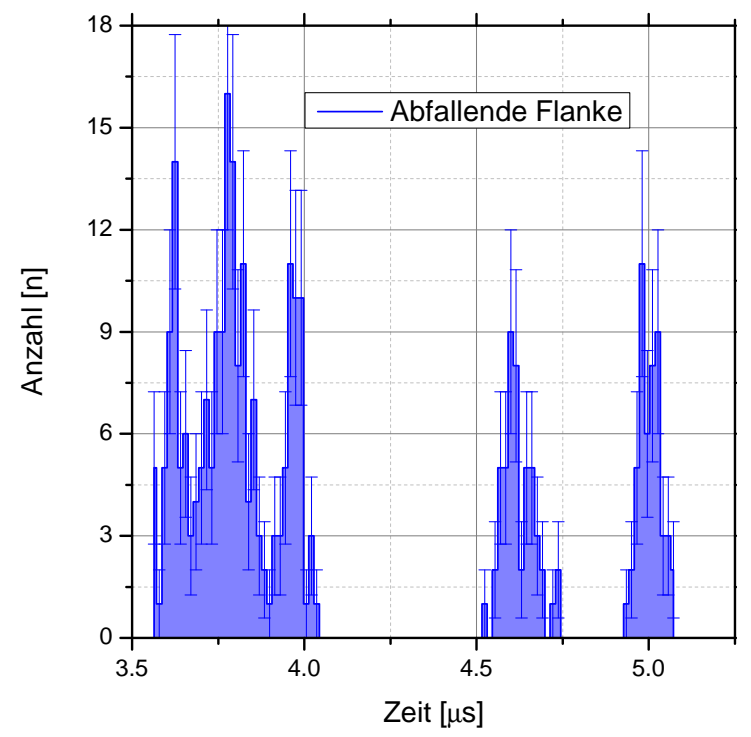

(b)

Abbildung 3.13: Statistische Verteilung der ansteigenden (a) und abfallenden Flanke (b) bei einer Chopper-Frequenz von $f_{c}=300(1) \mathrm{Hz}$. Die bei der abfallenden Flanke im höheren Bereich befindlichen Ereignisse können von hochfrequenten Modulationen der Intensität in Verbindung mit der Abtastrate des Oszilloskops herrühren.

Abbildung 3.14: Ausschnitt eines Augendiagramms aus einer Überlagerung aus ansteigender und abfallender Flanke bei einer Chopper-Frequenz von $f_{c}=$ 300(1) Hz. Der zeitliche Jitter führt zu einer Aufweitung, welche deutlich größer als die eigentlichen Flankenzeiten ist. Die Modulationen der Intensität (oben links) rühren von der Abtastung des Oszilloskops und des gepulsten Lasersignals.

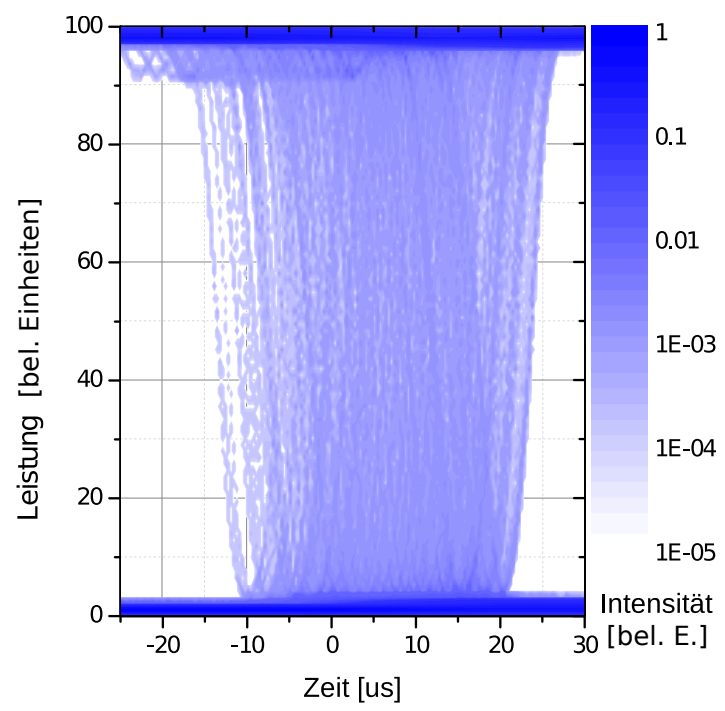


beschränkt. Beispiele sind hier periodischer Jitter durch zyklische Modulation, datenabhängiger Jitter oder Jitter durch asymmetrische Flanken. Um die Größe des deterministischen Jitters abzuschätzen kann eine Dual-Dirac-Analyse $(\delta-\delta)[289]$, welche in der Signalverarbeitung eine breite Anwendung findet, durchgeführt werden. Dafür werden im einfachsten Fall zwei Normalverteilungen (Anteil des zufälligen Jitters) an die linke und rechte Flanke der Verteilung angepasst, wobei der Abstand der jeweiligen Mittelwerte dem Wert des deterministischen Anteils entspricht. Die Größe des Jitters hängt maßgeblich von der zugrunde liegenden Referenz ab. Typische Analysen betreffen den Period-to-Period Jitter (PP-Jitter), welcher die Länge der Perioden in Bezug zu einer durchschnittlichen Periodendauer vergleicht, wie auch den Cycle-to-Cycle Jitter, bei dem die zeitliche Differenz aufeinander folgender Perioden oder auch längerer Zyklen verglichen wird [287]. Bei beiden Varianten ergibt sich die Referenz aus dem eigentlichen Signal selbst, sodass keine externe Uhr benötigt, jedoch aber auch nicht berücksichtigt werden kann. Im Gegensatz dazu steht der Time-Interval Jitter, welcher das Signal mit einer idealen Referenz vergleicht und aus den Differenzen zu dieser gebildet wird [287]. In Abbildung 3.15(a) ist der PP-Jitter (blau) des Chopperrads bei einer Frequenz von $f_{c}=300 \mathrm{~Hz}$ abgebildet. Deutlich zu erkennen sind zwei um $\pm 20 \mu$ s getrennte Regionen ohne gezählte Ereignisse im inneren Bereich, was auf einen stark ausgeprägten diskreten deterministischen Anteil im Jitter, sowie eine mögliche asymmetrische Flankenverteilung hinweist. Dieser Jitter kann als obere Grenze für kurzzeitige Messungen betrachtet werden, da Trigger und eigentliches Signal in einer sinnvollen Anordnung höchsten eine Periode voneinander entfernt sind. Im Experiment selbst wird das TTL-Signal des Choppercontrollers als Referenz verwendet. Der Jitter zu diesem und den entsprechenden Flanken des Signals ist ebenfalls in der Abbildung 3.15 (a) dargestellt (rot). Die Amplitude verglichen mit dem PP-Jitter ist in etwa nur halb so groß. Auch hier zeigen sich mehrere sehr diskrete Bereiche. Zufälliger sowie deterministischer Jitteranteil verhalten sich mit der Periodendauer linear (siehe Abbildung 3.15(b)). Zusammen sind sie in etwa eine halbe Größenordnung größer als die eigentlichen ansteigenden bzw. abfallenden Flankenzeiten, wodurch sie die zeitliche Auflösung bei Messungen mit dem Chopperrad dominieren.

Im Hinblick zu den eigentlichen Periodenzeiten bei einer Betrachtung von 300 aufeinander folgenden Perioden treten die diskret voneinander getrennten Regionen ebenfalls deutlich hervor (siehe Abbildung 3.16(a)). Dabei lassen sich sechs unterschiedliche Bereiche mit jeweils ähnlicher Integrationsfläche ermitteln. Diese korrespondieren gut mit den sechs Fenstern, welche das Chopperrad besitzt. Eine zeitliche Auftragung offenbart eine periodische Modulation mit einer Länge von sechs Ereignissen (siehe Abbildung 3.16(b)). Der starke deterministische Jitteranteil rührt daraus folgend von einer Unwucht des Chopperrads her. Zudem sind auch langsamere Schwankungen der Periodendauer (sog. Wandern 286]), sowie leichte Drifts zu erkennen. Diese Drifts lassen sich durch eine lange Aufwärmzeit sowie durch eine nachträgliche Korrektur, wie später in Kapitel 4.3 beschrieben, mindern. 


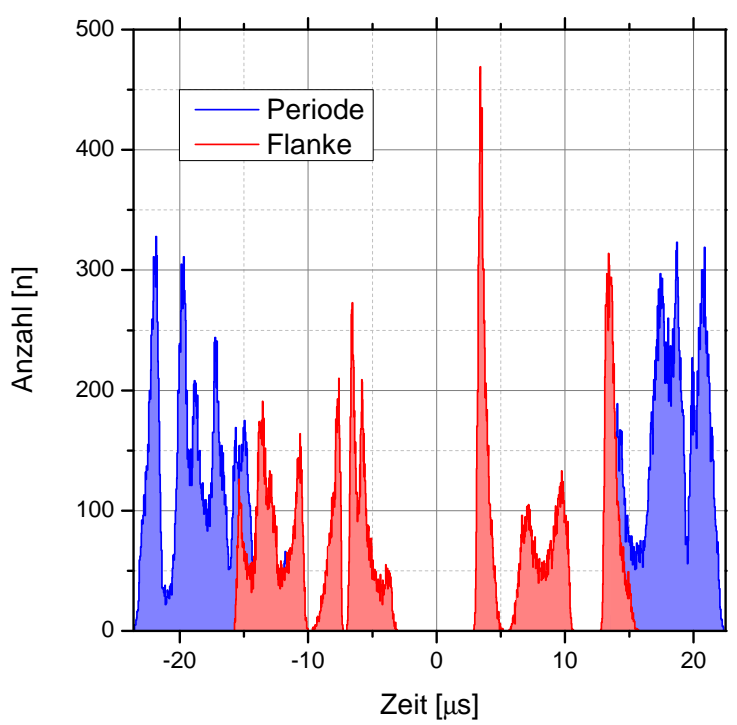

(a)

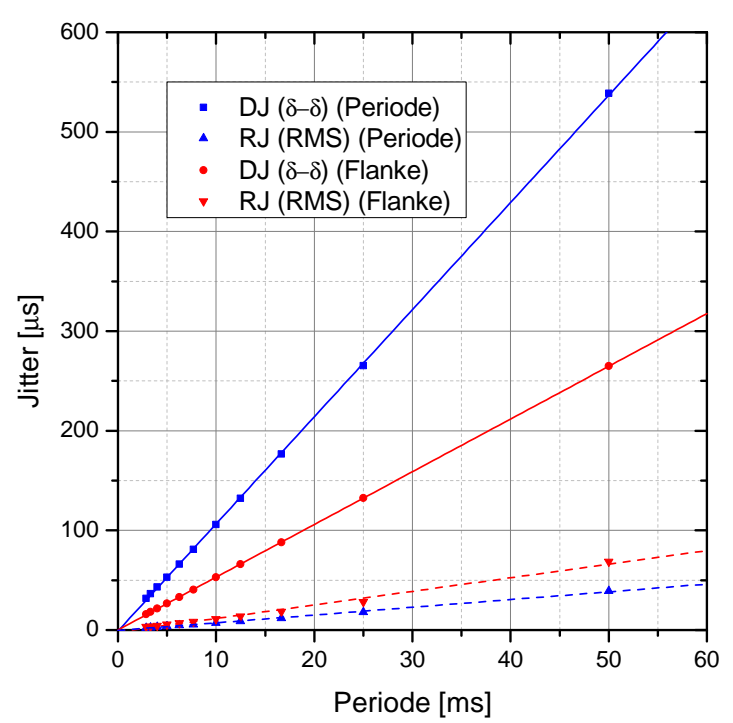

(b)

Abbildung 3.15: (a) Zeitlicher Jitter zwischen den Periodendauern des Lasers und der Chopper-Uhr (blau) und den jeweils korrespondieren Flanken (rot). Durch Unwucht im Chopperrad wird ein starker periodischer Anteil hervorgerufen, welcher für verschiedene Maxima sorgt und den Jitter nach außen drängt. (b) $\operatorname{DJ}(\delta-\delta)$ und RJ für periodengebundene (blau) sowie flankenbezogene (rot) Betrachtung mit jeweiligen linearen Anpassungen.

\subsubsection{Fokussiereinheit}

Die letzten Elemente vor der Probenkammer sind ein doppeltes Filterrad mit Neutraldichtefiltern sowie eine Fokussiereinheit bestehend auch drei Irisblenden und einer plankonvexen Sammellinse. Mit den vorhandenen Filtern können leistungsabhängige Messungen mit einer Abschwächung der Primärleistung auf 1\%o bis hin zur vollen Leistung durchgeführt werden. Die Schrittweite zwischen verschiedenen Filterkombinationen beträgt 0.1 OD. Da für verschiedene Harmonische als auch für die Fundamentale unterschiedliche Strahlwege durchlaufen werden müssen, befinden sich vor dem seitlichen Fenstereingang der Probenkammer drei linear in einer Käfighalterung ausgerichtete Irisblenden. Diese ermöglichen eine schnelle Vorjustage als auch das Abblenden ungewollter verarmter Restleistung bei der Erzeugung Harmonischer. 


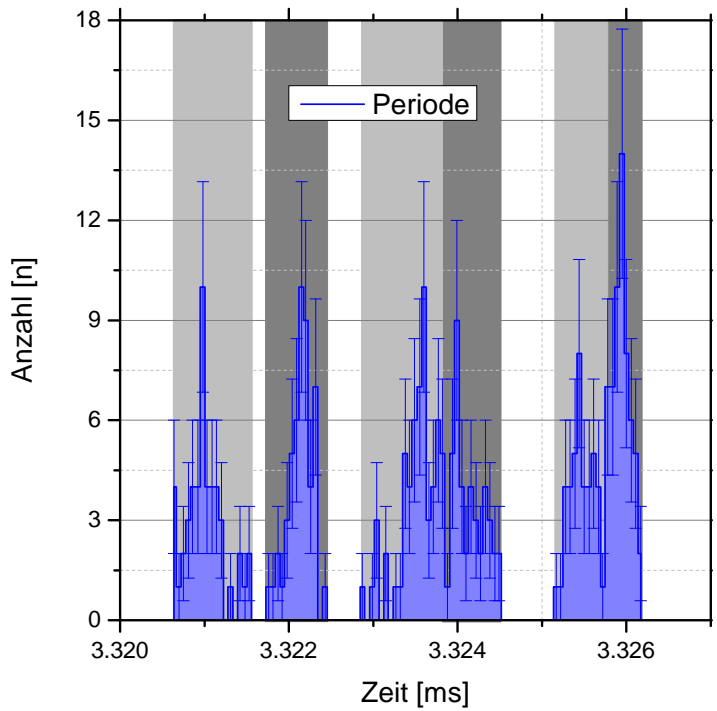

(a)

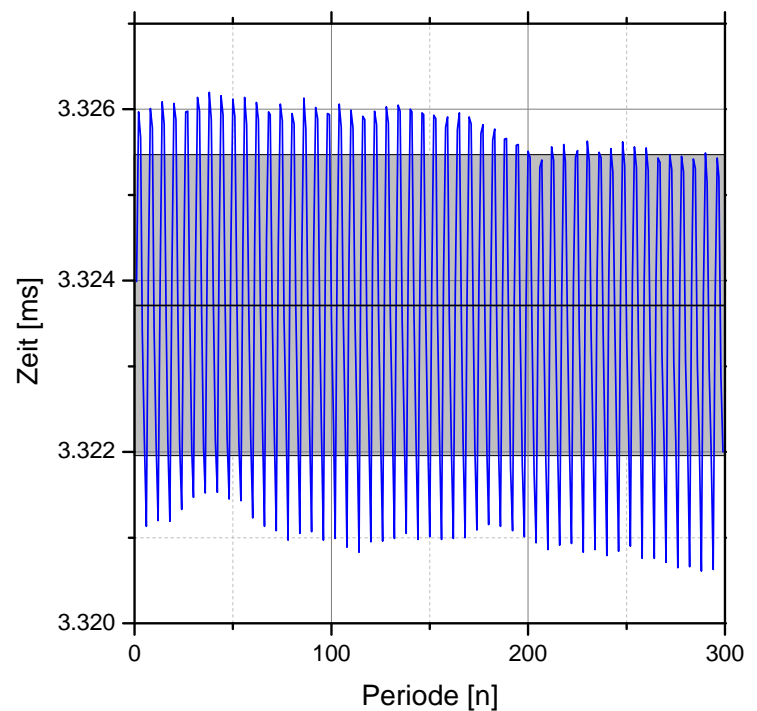

(b)

Abbildung 3.16: (a) Statistische Verteilung der Periode bei einer ChopperFrequenz von $f_{c}=300(1) \mathrm{Hz}$. Die grau hinterlegten Bereiche markieren etwa gleich viele Ereignisse, welche zu den sechs Fächern des Chopperrads korrespondieren. (b) Zeitliche Variation der Perioden mit einer zyklischen Dauer von sechs und einer Standardabweichung von $1.8 \mu$ s. Des weiteren zeigen sich langsamere Variationen und zeitliche Drifts.

\subsection{Probenkammer}

Für die eigentliche Analyse der Proben steht eine Vakuumkammer, welche mit einem geschlossenem Helium-Kryostat ausgestattet ist zur Verfügung. An der Kammer selbst befinden sich verschiedene gegenüberliegende Flansche jeweils angebracht unter einem Winkel von $60^{\circ}$. Einer dieser Flansche dient als Eintrittsfenster für den anregenden Laser. Der gegenüberliegende Flansch besitzt ebenfalls ein Fenster hinter welchem eine Fingerkamera mit vorgeschaltetem Objektiv angebracht ist. Diese dient der Überwachung während der Justage der Proben als auch der Laserpositionierung. Des weiteren befindet sich zentral von oben eingeführt eine Elektronenkanone (EQ 22, SPECS Surface Nano Analysis $\mathrm{GmbH}$ ) für Kathodolumineszenzexperimente. Das für solche Experimente nötige Vakuum wird von einer Turbomolekularpumpe (Pfeiffer Vacuum GmbH) mit vorgeschalteter Membranpumpe (vacuubrand $G M B H+C O$ $K G$ ) bereitgestellt. In der vorliegenden Konfiguration lassen sich so Basisdrücke von bis zu $p_{B}=6 \cdot 10^{-7}$ mbar bei Raumtemperatur erreichen. Ein limitierender Faktor ist hierbei das Austrittsfenster zur Beobachtung der Lumineszenz, welches zugleich zur Beladung mit bzw. Entnahme von Proben dient und somit aus praktikablen Gründen 
lediglich mit einer Gummidichtung versehen ist. Bei tiefen Temperaturen können sich so bei zu hohen Drücken Restbestandteile in der umgebenen Atmosphäre auf der Probe absetzten, was im Falle von Kathodolumineszenzmessungen aufgrund der geringen Eindringtiefe der Elektronen zu einer Abschwächung des Messsignals führt. Bei Messungen mit dem Laser spielt dieser Effekt nur eine untergeordnete Rolle, da bezogen auf die Dicke der sich ablegenden Schichten nur relativ niedrige Absorptionskoeffizienten, mit Ausnahme von Ozon, was jedoch nur in sehr geringer Konzentration auftreten sollte, vorherrschen [273]. Die Abkühlung der Proben auf bis zu $12 \mathrm{~K}$ wird mit einem geschlossenem Helium-Kryostat realisiert. Der Kühlkopf (Model 22, CTI-Cryogenics) ist mit einem Wellschlauch flexibel an den vorderen Part der Kammer angeschlossen (siehe Abbildung 3.17). Der zweistufige Kühlfinger ragt dabei in den vorderen Teil hinein. Die erste Stufe, welche auf etwa $77 \mathrm{~K}$ herunter gekühlt werden kann [290] ist zudem noch mit einem Wärmeschild zur Reduzierung der von außen eindringenden Wärmestrahlung auf die zweite Stufe, sowie den Probenhalter versehen. Der Kühlkopf selbst ist weiterhin noch mit dem zugehörigen Heliumkompressor (8200 Compressor, CTI-Cryogenics) verbunden. Die Temperatur nahe der Position des eigentlichen Probenhalters wird mit Hilfe einer Diode und einer am Kühlfinger befestigten Heizwendel über einen externen Controller (331 Temperature Controller, Lake Shore Cryotronics, Inc.) gesteuert. Durch die flexible Aufhängung kann der gesamte hintere Teil inklusive Kühlkopf und dem daran befestigten Probenhalter mittels einer motorisierten XY-Steuerung in horizontaler als auch vertikaler Richtung bewegt werden. Dies ermöglicht eine Positionierung der Proben ohne den eigentlichen Strahlverlauf der gesamten Experimentieranordnung ändern zu müssen. Eine detaillierte Beschreibung der Verschaltung kann in [259] gefunden werden. Im Falle einer benötigten elektrischen Kontaktierung der Proben befindet sich an der Kammer noch ein weiterer Flansch mit zwei koaxial durchgeführten BNC-Buchsen. Diese erlauben elektrische Signalübertragungen bis zu einem Frequenzbereich von $4 \mathrm{GHz}$ [291].

Zur Fixierung der Proben in der Kammer existieren zwei Typen von Halterungen. Für Flüssigkeiten wurde eine Halterung konstruiert, welche Einweg- oder auch eine dazu passende Quarzküvette aufnehmen kann (siehe Abbildung 3.18(a)). Tieftemperaturexperimente sind aufgrund der schlechten Wärmekopplung als auch dem Umstand der Undichtigkeit der Küvetten bzgl. einer vorzeitigen Verdampfung der meisten Flüssigkeiten bei starkem Druckabfall geschuldet, nicht möglich. Pulver-, Dünnschicht oder kleine kristalline Proben können mit einem sauerstoffarmen Kupferhalter (siehe Abbildung 3.18(b)) an den Kühlfinger angebracht werden. Die Proben werden dafür über Graphitpads oder mit Leitsilber an diesem befestigt. 


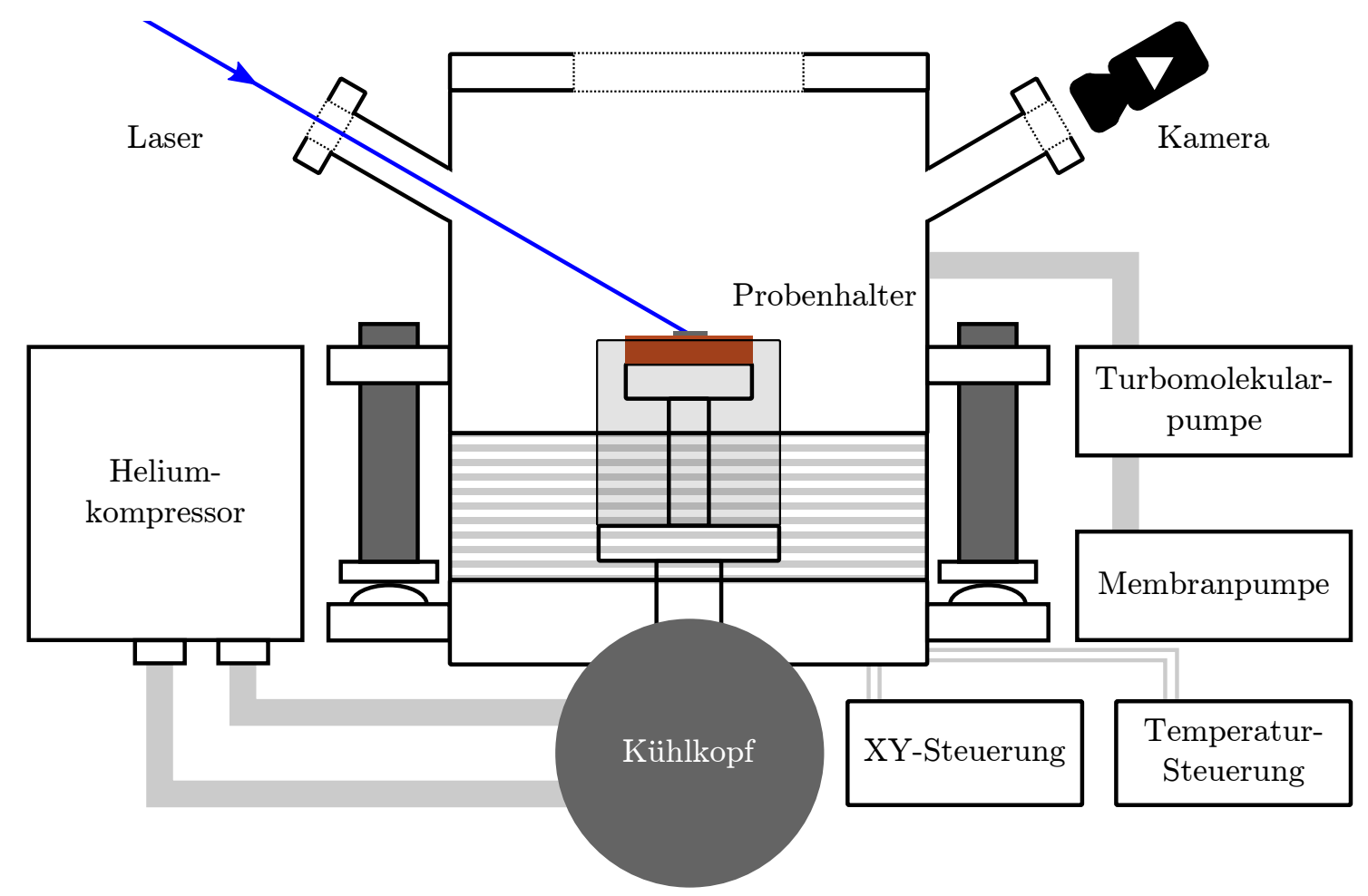

Abbildung 3.17: Schematische Darstellung der Probenkammer und ihrer Peripheriegeräte für Photolumineszenzmessungen. Der Probenhalter samt Kühlfinger und Kühlkopf, welche wiederum am hinteren Teil der Kammer befestigt sind, können unter Nutzung der motorisierten XY-Steuerung in der Probenkammer horizontal und vertikal bewegt werden. Ausgeglichen wird diese Verschiebung mit Hilfe eines Edelstahl-Wellschlauchs. 


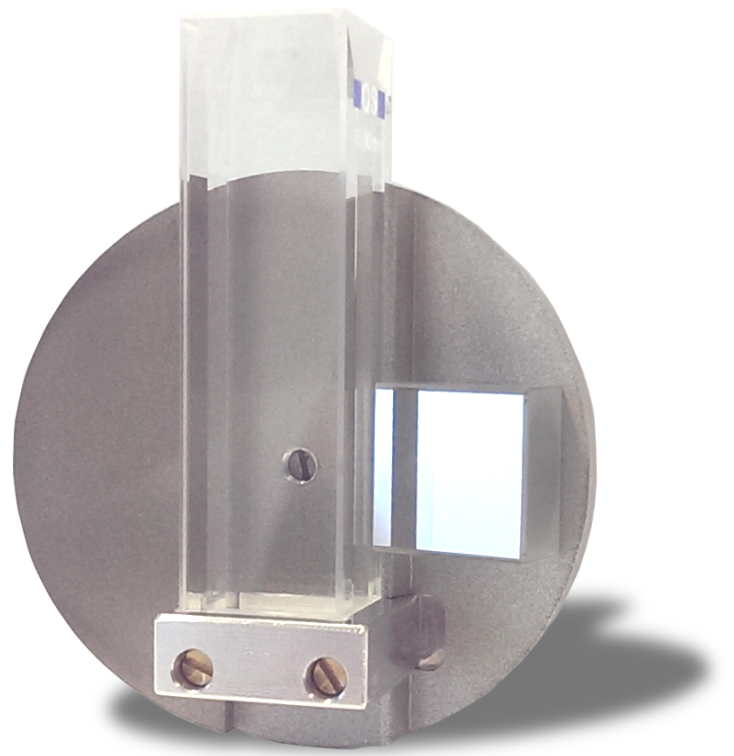

(a)

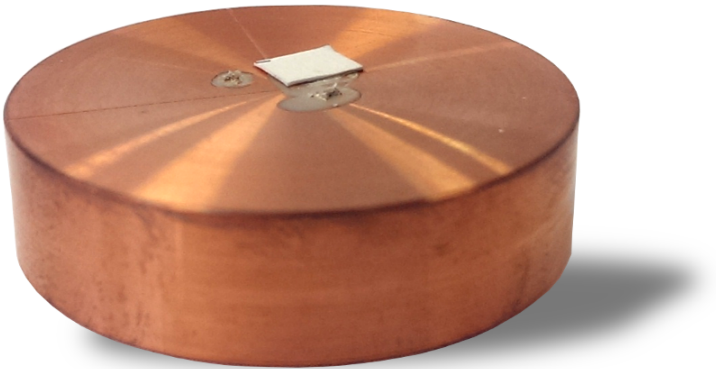

(b)

Abbildung 3.18: (a) Halterung samt Quarzküvette zur Untersuchung von Flüssigkeiten. Der Laser trifft hierbei zunächst auf den nebenstehenden Spiegel auf und wird dann planparallel durch die Küvette geführt. (b) Probenhalter aus sauerstoffarmen Kupfer für Pulver-, Dünnschicht- oder kleine kristalline Proben. An der Oberfläche für bestmögliche Wärmekopplung mit Leitsilber direkt aufgebracht befinden sich zwei kristalline HPHT-AlN-Proben. Für die Vorjustage befindet sich zusätzlich ein Stück Papier am Probenhalter.

\subsection{Optisches Messsystem}

Die eigentliche Analyse der Proben kann über zwei verschiedene Wege im Messaufbau erfolgen. Zum einen befindet sich ein $0.3 \mathrm{~m}$-Spektrometer mit anschließendem StreakSystem direkt gerade vor der Probenkammer, zum anderen ein senkrecht dazu aufgebautes $1 \mathrm{~m}$-Spektrometer mit verschiedenen Detektoren für hochaufgelöste Messungen. Ausgewählt werden können die jeweiligen Strahlbahnen über einen optional einbringbaren planen um $45^{\circ}$ zur Probenkammer rotierten AluminiumSpiegel. Die Fokussierung Richtung Eingangsspalt zum 0.3 m-Spektrometer erfolgt über zwei plankonvexe 2"-Linsen mit jeweils $150 \mathrm{~mm}$ Brennweite, wobei die erste Linse als Kollimator fungiert. Unmittelbar vor dem Spektrometer wird mit Hilfe eines Langpassfilters die von der Probe reflektierte Restleistung des Lasersignals zum Schutz der darauf folgenden Komponenten unterdrückt. Im Falle von breitbandigen Signalen oder Up-Conversion-Experimenten kommen an dieser Stelle auch weitere Filter zur Ausblendung bestimmter spektraler Bereiche zum Einsatz. Vor dem 1 mSpektrometer werden die vorhin benannten Linsen zur Erzeugung eines Abbilds, an 
dessen Position auch leicht Filter eingebracht werden können, eingesetzt. Daraufhin wird mit einem Linsensystem bestehend aus einer konvexen und einer konkaven Linse das einfallende Lumineszenzlicht auf den Eingangsspalt dieses Spektrometers fokussiert und dabei auf den passenden Divergenzwinkel eingestellt.

Im folgenden Kapitel wird nun näher auf die einzelnen Komponenten im Analyseweg des experimentellen Messaufbaus eingegangen.

\subsubsection{Spektrometer}

Wie weiter oben schon beschrieben, befinden sich zwei Spektrometer zur spektralen Aufspaltung der Lumineszenzsignale im Strahlengang, wobei sich die Anwendungsbereiche schon allein wegen der unterschiedlichen fokalen Längen und der an ihnen angeschlossenen weiteren Peripherie stark unterscheiden. Das schon vor dieser Arbeit vorhandene $1.0 \mathrm{~m}$-Spektrometer soll hier nur kurz vorgestellt werden. Für detailliertere Ausführungen sei auf die Dissertation von Vetter 259] verwiesen.

\section{$1.0 \mathrm{~m}$-Spektrometer}

Bei diesem Spektrometer handelt es sich um ein Czerny-Turner Spektrometer 292 (1000M, SPEX Industries) mit einer Fokallänge von $1.0 \mathrm{~m}$. Das im Spektrometer befindliche Gitter kann per Hand ausgetauscht werden. Es liegen drei Blazegitter mit jeweils $100 \mathrm{~g} / \mathrm{mm}, 600 \mathrm{~g} / \mathrm{mm}$ und $1200 \mathrm{~g} / \mathrm{mm}$ vor. Im Eingangsbereich direkt vor dem Spalt befindet sich ein zusätzliches Filterrad zur Unterdrückung spektraler Replika höherer Ordnung, welche bei Proben mit breitbandigem Lumineszenzverhalten auftreten können. Nach dem Spalt folgt ein Shutter. Dieser kann für automatische Hintergrundmessungen mit anschließender Subtraktion genutzt werden. Innerhalb des Spektrometers lassen sich per elektronischer Ansteuerung eines klappbaren Spiegels zwei verschiedene Ausgänge anwählen. Ohne Spiegel fällt das Signal auf einen mit flüssigem Stickstoff gekühlten CCD-Chip (CCD30-11, e2v Technologies $P L C)$, mit welchem breitbandige Übersichtsspektren gewonnen werden können. Durch den relativ geringen dynamischen Bereich $(<80000$ [259]) gestaltet sich die Auswertung von gleichzeitigen Signalen mit Intensitätsunterschieden größer als zwei Größenordnungen schwierig. Bei ausgeklapptem Spiegel wird das Licht auf einen seitlichen Ausgang des Spektrometers abgebildet. Dort befindet sich ein weiterer vertikaler Spalt, wodurch das Spektrometer als Monochromator fungiert. Angeschlossen werden können hier ein Photomultiplier (R928, Hamamatsu Photonics K.K.) oder ein InGaAs-Detektor (IGA, Electro Optical Systems GmbH). Der Photomultiplier eignet sich für Messungen im UV- als auch im sichtbaren Bereich und zeichnet sich durch eine hohe Dynamik von $>10^{6}$ aus [259]. Betrieben wird jener typischerweise im Photon-Counting-Modus. Hierbei erzeugt jedes einzelne Photonenereignis einen elektrischen Puls, sodass mit ihm auch zeitaufgelöste Messungen durchgeführt 
werden können. Im Gegensatz dazu eignet sich der InGaAs-Detektor mit einem spektralen Bereich von 800 - 1700 nm [293] für Experimente im nahen Infrarot.

Im Rahmen dieser Arbeit wurden auch einzelne Übersichtsspektren mit diesem Spektrometer aufgenommen. Dabei wurde der Photomultiplier R928 als Detektor verwendet. Da die einzelnen Komponenten innerhalb des Spektrometers eine stark wellenlängenabhängige Sensitivität aufweisen, wurde diese unter Berücksichtigung der Effizienzen der im Spektrometer verbauten Spiegel, des verwendeten Gitters und der Photokathode des Photomultipliers abgeschätzt. Die daraus resultierende gesamte Effizienz ist in Abbildung 3.19 dargestellt. Eine Korrektur bezüglich der vor dem Spektrometer befindlichen Eingangsoptik findet hingegen nicht statt. Für das optische System der Streak-Kamera erfolgt eine eben solche Abschätzung in Kapitel 4.2 .

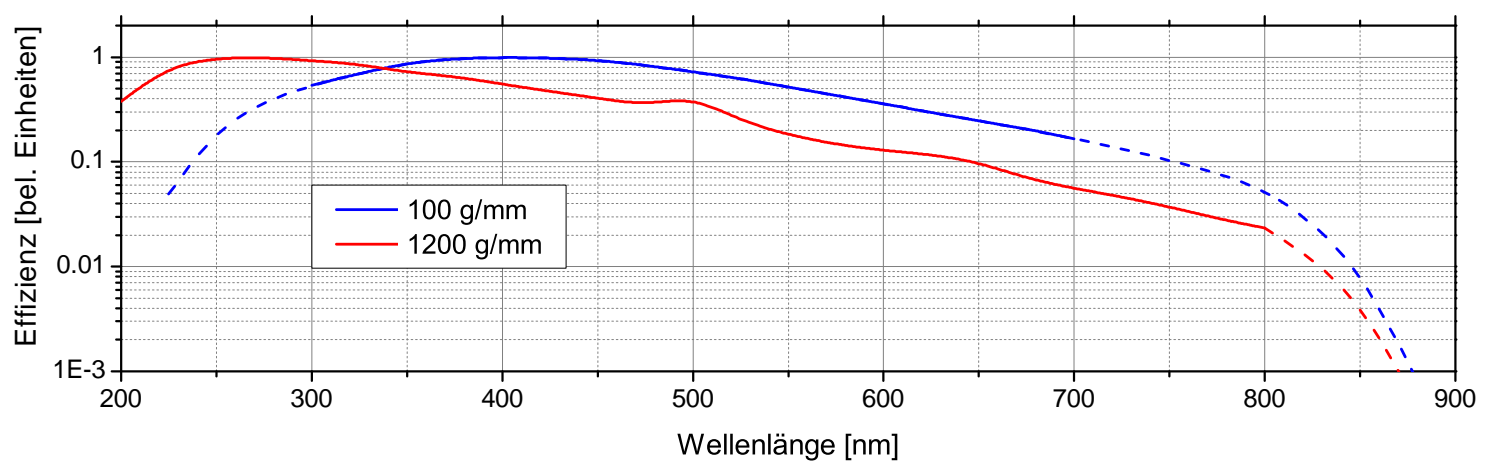

Abbildung 3.19: Abschätzung der maximal erreichbaren Effizienz des $1.0 \mathrm{~m}$ Spektrometers unter Berücksichtigung der verbauten Spiegel (Aluminium) bei einer Verwendung des $100 \mathrm{~g} / \mathrm{mm}$ - beziehungsweise des $1200 \mathrm{~g} / \mathrm{mm}$-Gitters und des R928-Photomultipliers für unpolarisiertes Licht. Die Daten für die Gitter und den Photomultiplier stammen aus den Referenzen [294,295] respektive [296]. Bei den gestrichelt dargestellten Anteilen handelt es sich um eine lineare Extrapolation der Gitterdaten, da jene vom Hersteller nur in einem kleinerem Wellenlängenbereich vorliegen.

\section{$0.3 \mathrm{~m}$-Spektrometer}

Auch bei diesem Spektrometer handelt es sich um eines in der von Czerny und Turner 292] vorgeschlagenen Bauweise. Die Fokallänge beträgt jedoch nur $0.3 \mathrm{~m}$. Im Gegensatz zum weiter oben beschriebenen Spektrometer können hier jedoch bis zu drei Gitter simultan an einen im Inneren befindlichen drehbar gelagerten Turm angebracht werden. Die Auswahl eines Gitters als auch die Einstellung der gewünschten Verkippung jenes erfolgt über die integrierte Messsoftware, welche auch zur Ansteuerung der Komponenten für die Streak-Kamera Anwendung findet. Der sich im Eingangsbereich befindliche motorisierte Spalt kann auf Größen zwischen 
5 - $3000 \mu \mathrm{m}$ eingestellt werden. Das einfallende Licht wird direkt hinter dem Eingangsspalt über einen planen Spiegel umgelenkt und anschließend mit Hilfe zweier 4" -Hohlspiegel auf den Gitterturm zunächst kollimiert und danach auf die Austrittsebene fokussiert. Alle Spiegelflächen sind dabei mit einer speziellen $\mathrm{Al}+\mathrm{MgF}_{2}$ Beschichtung versehen, die eine höhere Reflektivität verglichen zu reinem Aluminium im UV-Bereich aufweist [297]. Zur einfacheren Justage wurde das Spektrometer im Rahmen dieser Arbeit mit einem in den Strahlengang ein- und ausfahrbaren Periskop ausgestattet. An dieses wurde eine Kamera zur direkten Beobachtung des Spalts vom Inneren des Spektrometers heraus angebracht. Hierdurch kann bei korrekter Fokussierung die zu untersuchende Probe beobachtet werden.

Bei den Gittern handelt es sich um drei etwa $10 \mathrm{~cm}$ große reflektive Blazegitter mit jeweils $50 \mathrm{~g} / \mathrm{mm}, 300 \mathrm{~g} / \mathrm{mm}$ und $900 \mathrm{~g} / \mathrm{mm}$. Die beiden erstgenannten besitzen flache Blazewinkel $\Theta_{B}$ (Winkel zwischen Oberfläche der Kerben und der Ebene des Gitters [301]) von $0.86^{\circ}$ bzw. $2.4^{\circ}$, was zu einem relativ uniformen Reflexionsverhalten zwischen parallel und senkrecht polarisiertem Licht führt. Des weiteren verhält sich die Effizienz vom Maximum aus gesehen in beide Richtungen monoton (siehe Abbildung 3.20(a) und (b)). Das dritte Gitter besitzt einen Blazewinkel von $\Theta_{B}=14.3^{\circ}$, was zu starken Unterschieden zwischen den beiden Polarisationsrichtungen führt (siehe Abbildung 3.20(c)). Bei solchen Gittern lässt sich das Reflexionsmaximum näherungsweise aus dem Linienabstand $d$, der Ordnung $m$ (hier $m=1$ ) und dem Blazewinkel in Littrow-Anordnung (Licht fällt lotrecht auf die Oberfläche der Kerben) folgendermaßen bestimmen [301]:

$$
\lambda_{B}=\frac{2 d}{m} \sin \Theta_{B} .
$$

Dies ergibt für die drei Gitter jeweilige Blazewellenlängen von $600 \mathrm{~nm}, 280 \mathrm{~nm}$ und $550 \mathrm{~nm}$, was anhand der in Abbildung 3.20 dargestellten Effizienzen nachvollzogen werden kann. Die Daten stammen hierbei aus Messungen in Littrow-Anodnung 298300], welche eine höhere Effizienz verglichen zu anderen Einfallswinkeln aufweisen [301]. Da beim Czerny-Turner-Spektrometer das Gitter verglichen zum einfallenden Strahl zum Erreichen verschiedener Wellenlängenbereiche gedreht werden muss, sind für Positionierungen weiter entfernt von der Littrow-Anordnung niedrigere Effizienzen zu erwarten.

Einer der wichtigsten Parameter eines spektroskopischen Aufbaus ist das mit der Apparatur zu erreichende Auflösungsvermögen. In Abbildung 3.22 ist die Halbwertsbreite drei verschiedener Quecksilberlinien (253.7 nm, $435.8 \mathrm{~nm}$ und $546.1 \mathrm{~nm}[302])$, erzeugt mit einer Niederdruck-Argon-Quecksilber-Dampflampe (LSP035, LOTQuantumDesign $\mathrm{GmbH}$ ), für unterschiedliche Eingangsspaltbreiten dargestellt. Die natürlichen Linienbreiten der ausgewählten Linien als auch ihre Verbreiterung durch verschiedene Isotope liegen noch unter dem Auflösungsvermögen des Spektrometers [302]. Somit können die beobachteten Linien als Anhalt für die tatsächliche spektrale Auflösung des Systems dienen. Zur Messung der $435.8 \mathrm{~nm}$ und $546.1 \mathrm{~nm}$ 


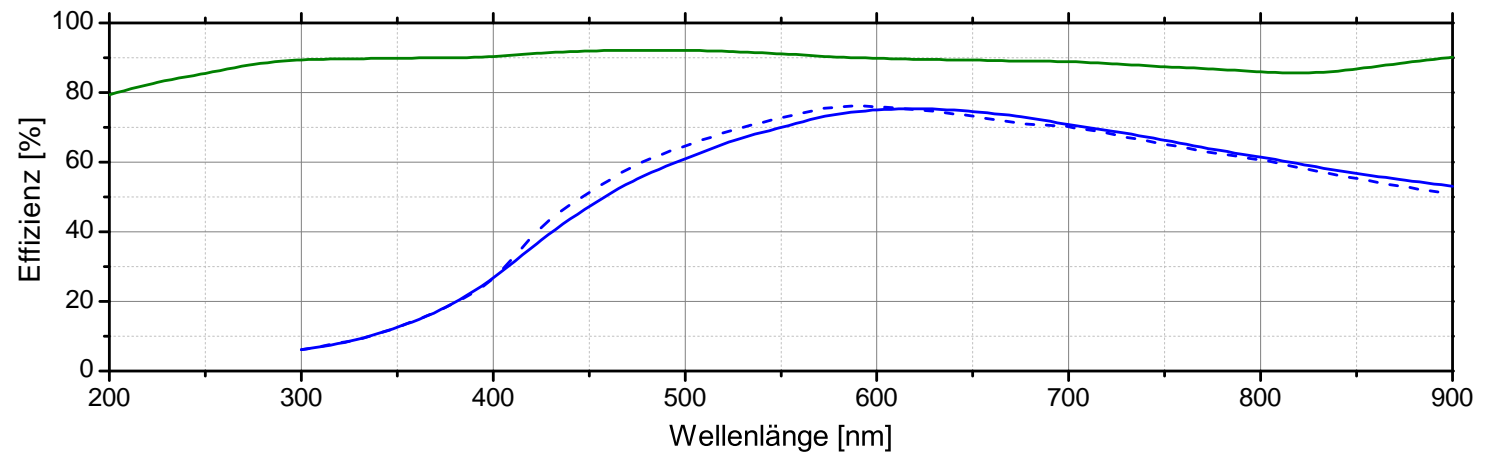

(a) Liniendichte: $50 \mathrm{~g} / \mathrm{mm}$, Blazewinkel: $0.86^{\circ}$, Blazewellenlänge: $600 \mathrm{~nm}$

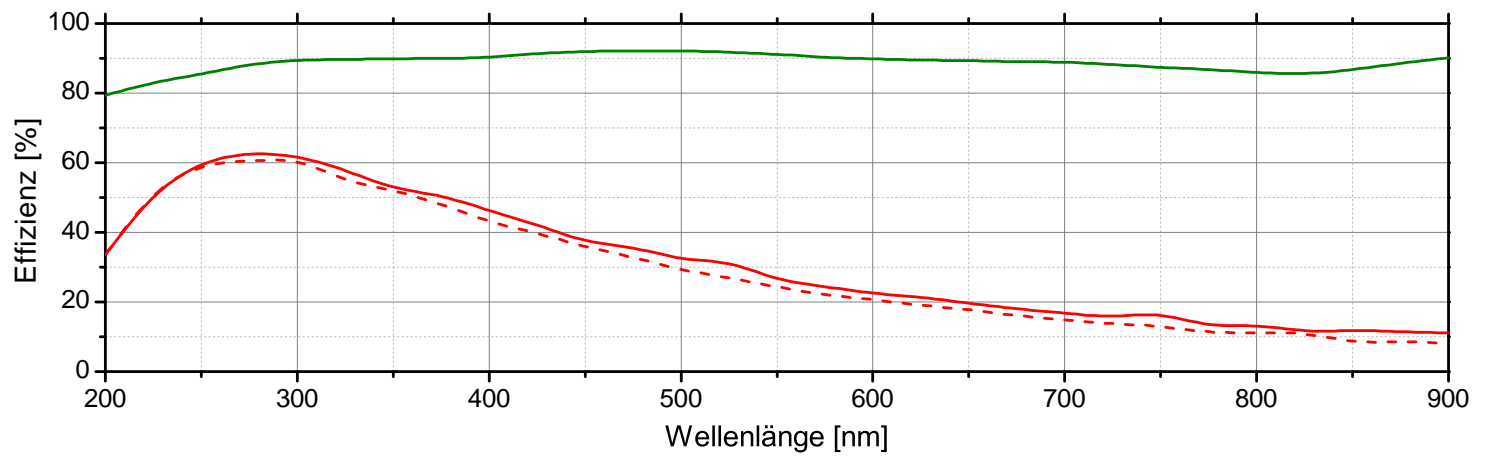

(b) Liniendichte: $300 \mathrm{~g} / \mathrm{mm}$, Blazewinkel: $2.4^{\circ}$, Blazewellenlänge: $280 \mathrm{~nm}$

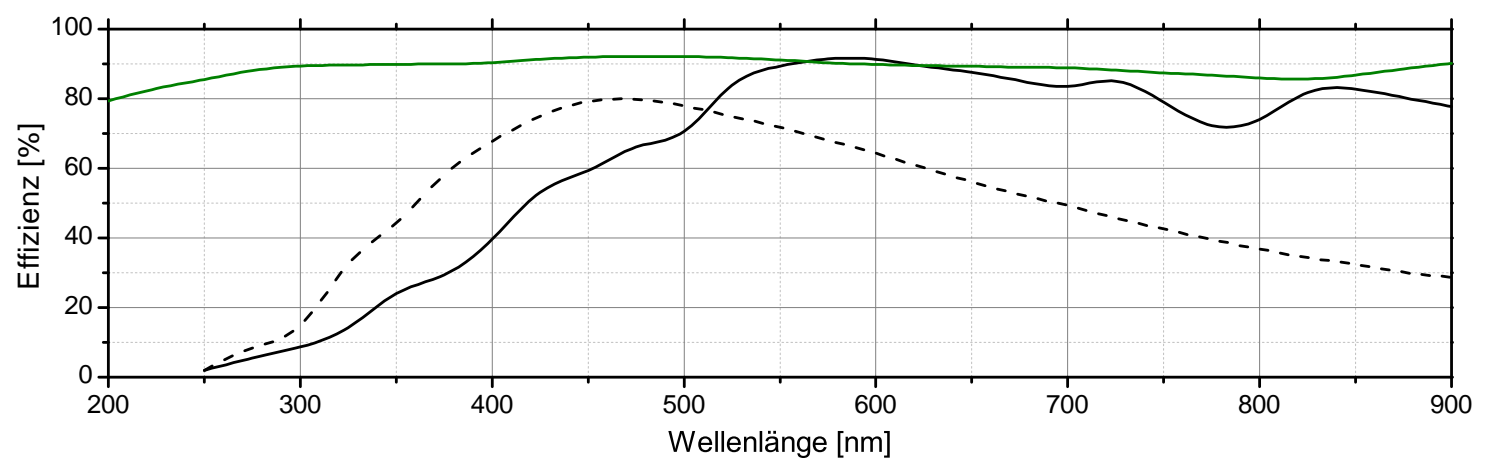

(c) Liniendichte: $900 \mathrm{~g} / \mathrm{mm}$, Blazewinkel: $14.3^{\circ}$, Blazewellenlänge: $550 \mathrm{~nm}$

Abbildung 3.20: Darstellung der Effizienz der drei Gitter im 0.3 m-Spektrometer bezogen auf Licht mit senkrechter (durchgehend) und paralleler (gestrichelt) Polarisation. Als Vergleich ist zudem der Reflexionsgrad von Aluminium (grüne Linie) angegeben. Die Daten der Gitter wurden aus [298 300] entnommen. Gemessen wurden die Gitter dabei in Littrow-Anordnung. 


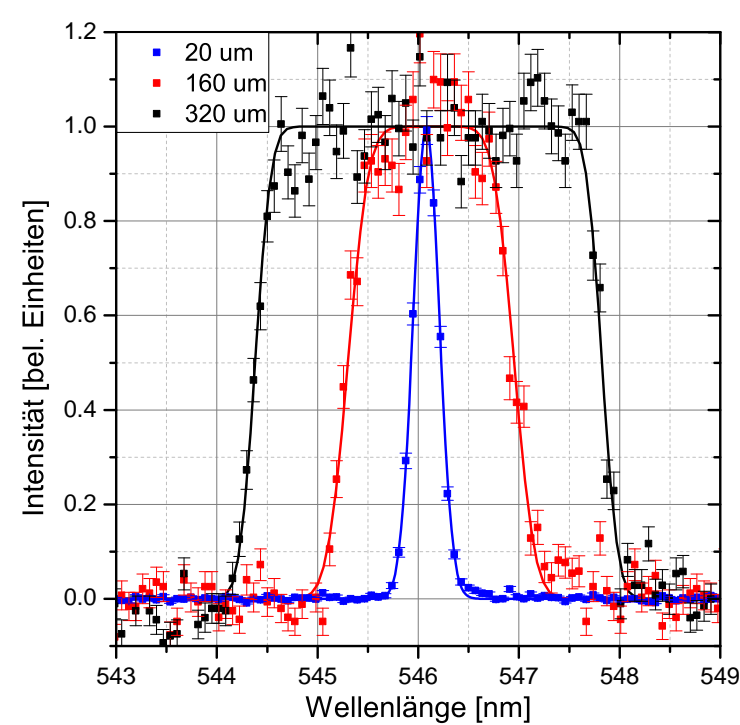

(a)

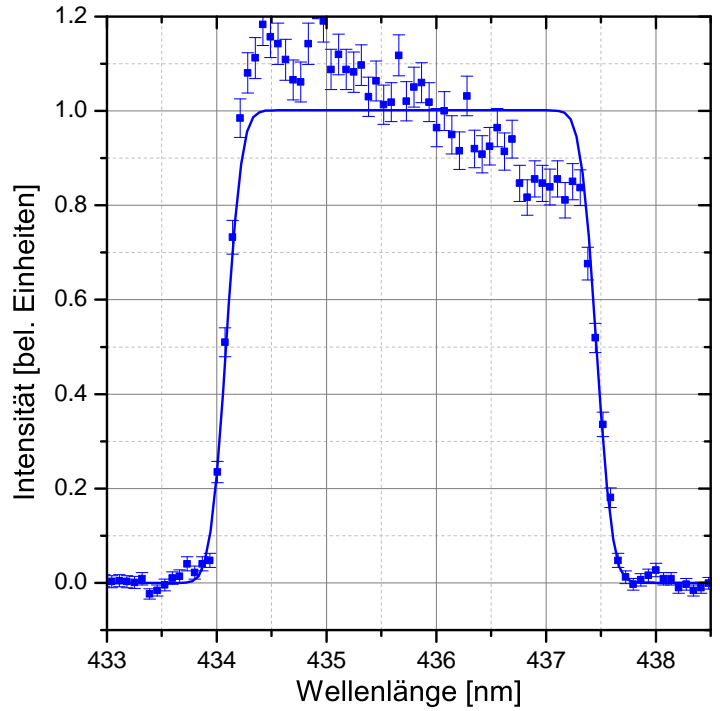

(b)

Abbildung 3.21: (a) Integrierte Spektren der $546.1 \mathrm{~nm}$ Quecksilberlinie aufgenommen mit dem $300 \mathrm{~g} / \mathrm{mm}$-Gitter für unterschiedliche Spaltbreiten. Die Spektren wurden nach Gleichung 3.9 angepasst, daraufhin der Untergrund abgezogen und auf das Maximum der angepassten Funktion normiert. (b) Eine ungleichmäßige Ausleuchtung des Eingangsspalts spiegelt sich in der Intensitätsverteilung wieder.

wurde ein Langpassfilter zur Unterdrückung der starken $253.7 \mathrm{~nm}$-Emission eingesetzt. Gemessen wurden die Spektren im Photon-Counting-Modus mit Sweep-Zeiten zwischen $200 \mu$ s und $1 \mathrm{~ms}$ (siehe hierfür Kapitel 3.3.4). Für kleine Spaltbreiten verhalten sich die Linien nahezu normalverteilt zum Maximum. Mit zunehmender Breite erweitert sich die Verteilung mit einem Plateau beim Intensitätsmaximum (siehe Abbildung 3.21(a)). Eine Normalverteilung mit Standardabweichung $\sigma$ gefaltet mit einer Rechteckfunktion der Breite $b$ :

$$
I(\lambda)=I_{0} \cdot\left[\operatorname{erfc}\left(\frac{\lambda-\lambda_{0}-\frac{1}{2} b}{\sqrt{2} \sigma}\right)-\operatorname{erfc}\left(\frac{\lambda-\lambda_{0}+\frac{1}{2} b}{\sqrt{2} \sigma}\right)\right]+c
$$

zeigt eine gute Abbildung der Quecksilberlinien. Die Parameter $I_{0}, \lambda_{0}$ und $c$ stehen hier jeweilig für den Intensitätsfaktor, die zentrale Wellenlänge und die Höhe des Untergrunds. Wie für große Spaltbreiten zu erwarten, verhält sich die Halbwertsbreite zu dieser linear. Minimale Breiten sind für alle Messungen bei einer Spaltgröße von etwa $20 \mu \mathrm{m}$ zu beobachten. Bei kleineren Weiten zeigen sich leicht verbreiterte Linien, zumeist vergleichbar mit denen bei einer Breite von $40 \mu \mathrm{m}$. Danach kann ein wie schon erwähnt linearer Anstieg beobachtet werden.

Die Öffnungsgröße des Eingangsspalts hat neben der Breite der Intensitätsmaxima auch noch Einfluss auf die Form dieser. Mit zunehmender Größe kann sich bei un- 
gleichmäßig ausgeleuchteten Proben dieser Umstand in den Messungen wiederfinden. Beispielhaft ist dies in Abbildung 3.21(b) dargestellt, bei der das Licht der Quecksilberlampe ungleichmäßig auf den Eingangsspalt fällt. Als weitere Störung kann in Abbildung 3.21(a) bei der Messung mit einem $20 \mu \mathrm{m}$-Spalt eine leichte Asymmetrie des Peaks mit erhöhter rechter Flanke möglicherweise hervorgerufen durch Koma 301 ausgemacht werden.

\subsubsection{Streak-Kamera}

Um zeitlich hochaufgelöste spektroskopische Aufnahmen realisieren zu können, kommt in der hier vorgestellten experimentellen Anordnung eine Streak-Kamera (auch Schmierbild-Kamera) zum Einsatz. Dabei handelt es sich um die Kernkomponente des während dieser Arbeit in Betrieb genommenen zeitaufgelösten Lumineszenzaufbaus. In Abbildung 3.23 ist das Funktionsprinzip konzeptionell dargestellt. Das einfallende vom Spektrometer spektral aufgeteilte Licht (siehe Kapitel 3.3.1) fällt auf einen im Gegensatz zu allen anderen im System verwendeten horizontalen Eingangsspalt. Die Lichtpulse können dabei verschiedene Intensitäten, Ankunftszeiten sowie Wellenlängen aufweisen. Hinter dem Spalt befindet sich eine Eingangsoptik (A1976-01, Hamamatsu Photonics K.K.), welche das Licht auf eine Photokathode (S-20, Hamamatsu Photonics K.K.) fokussiert. Die Eingangsoptik weist zwischen $400 \mathrm{~nm}$ und $1600 \mathrm{~nm}$ einen spektralen Transmissionsgrad von etwa $70 \%$ auf, während dieser kontinuierlich mit sinkender Wellenlänge auf einen Wert von $40 \%$ bei $200 \mathrm{~nm}$ abfällt 303]. Die Photokathode mit effektiven Ausmaßen von lediglich $0.15 \mathrm{~mm} \times$ $4.42 \mathrm{~mm}$ [304] befindet sich innerhalb einer evakuierten Vakuumröhre der eigentlichen Haupteinheit der Kamera (C10910-01, Hamamatsu Photonics K.K.). Sie besitzt zwischen $250 \mathrm{~nm}$ und $400 \mathrm{~nm}$ eine Quanteneffizienz von etwa $15 \%$, welche zu beiden Seiten gehend nahezu exponentiell bei $200 \mathrm{~nm}$ bzw. $820 \mathrm{~nm}$ auf einen Wert von $1 \%$ abfällt (siehe Abbildung 3.24(a)). Bei $900 \mathrm{~nm}$ liegt die Ausbeute nur noch bei etwa $0.1 \%$. Aufgrund des äußeren photoelektrischen Effekts 305,306 werden bei Einfall der Photonen Elektronen aus der Photokathode herausgelöst und mit Hilfe eines dahinter befindlichen Gitters weiter in die Vakuumröhre beschleunigt. Innerhalb der Vakuumröhre befindet sich eine Ablenkeinheit (Slow sweep unit M10913-01, Hamamatsu Photonics K.K.), die durch ein externes elektrisches Trigger-Signal angesteuert werden kann. Durch Anlegen einer Spannungsrampe werden Elektronen, die die Röhre zu unterschiedlichen Zeiten verglichen zum Trigger-Signal passieren auch verschieden stark in Richtung der vertikalen Achse abgelenkt. Die Rampenzeit kann aus einer Auswahl von 19 verschiedenen Zeiten zwischen $1.2 \mathrm{~ns}$ und 1 ms getroffen werden [304]. Der Vakuumröhre folgend gelangen die vertikal abgelenkten Elektronen daraufhin auf einen Sekundärelektronenvervielfacher in Form einer Mikrokanalplatte (MCP). Diese besitzt einen einstellbaren Verstärkungsfaktor zwischen 4 und etwa 9000 (siehe Abbildung 3.24(b)). Direkt im Anschluss an die MCP befindet sich ein Leuchtschirm, der beim Eintreffen der Elektronen über Kathodolumineszenz ein 


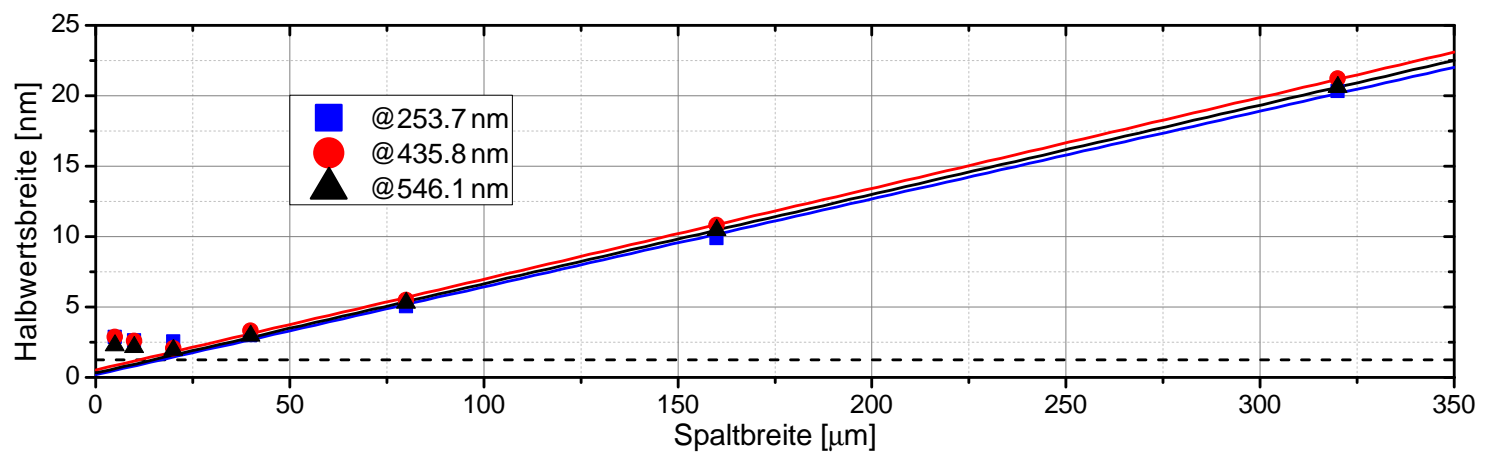

(a) Liniendichte: $50 \mathrm{~g} / \mathrm{mm}$, Blazewinkel: $0.86^{\circ}$, Blazewellenlänge: $600 \mathrm{~nm}$

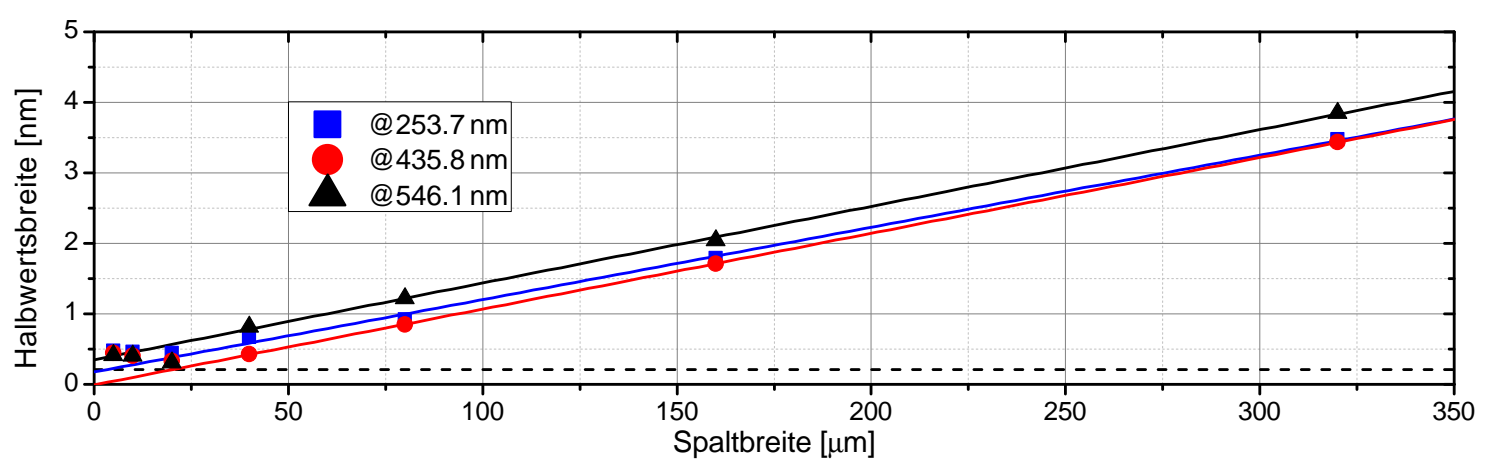

(b) Liniendichte: $300 \mathrm{~g} / \mathrm{mm}$, Blazewinkel: $2.4^{\circ}$, Blazewellenlänge: $280 \mathrm{~nm}$

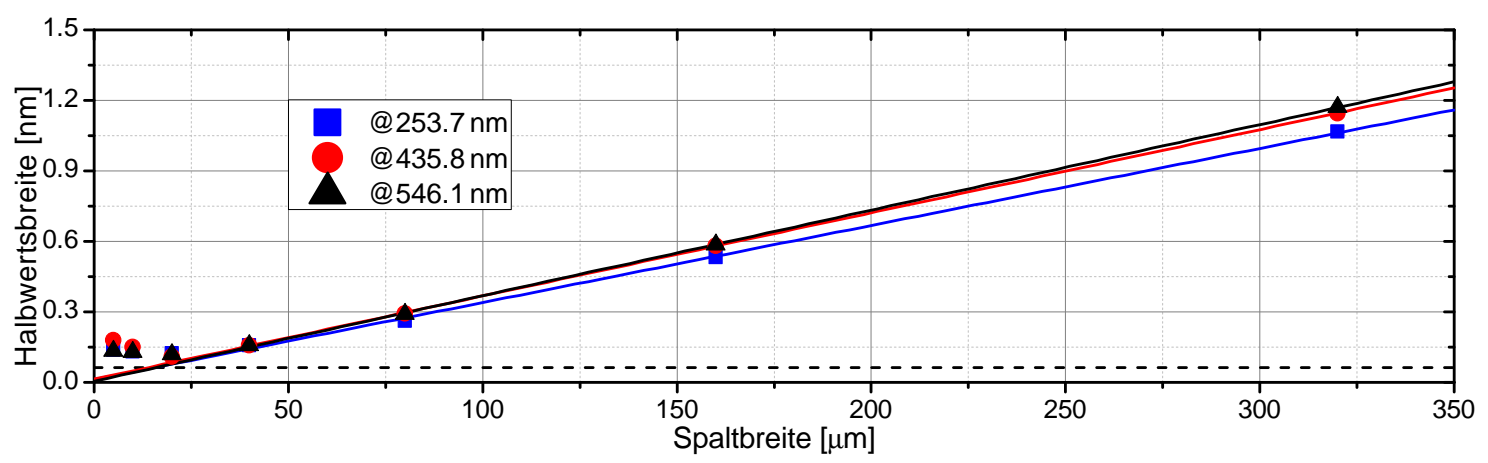

(c) Liniendichte: $900 \mathrm{~g} / \mathrm{mm}$, Blazewinkel: $14.3^{\circ}$, Blazewellenlänge: $550 \mathrm{~nm}$

Abbildung 3.22: Auflösungsvermögen der drei Gitter bei unterschiedlichen Spaltbreiten für verschiedene Wellenlängen ermittelt aus den Fits von Gleichung 3.9. Bei den Geraden handelt es sich um lineare Anpassungen für Spaltbreiten ab einer Größe von $40 \mu \mathrm{m}$. Die gestrichelte waagerechte Linie (am unteren Rand) entspricht der spektralen Breite dreier Pixel der Bildaufnahme. 
optisches Signal aus der Vakuumröhre emittiert. Somit ergibt sich ein zweidimensionales Feld, bei dem die resultierenden Leuchtpunkte in horizontaler Achse spektral aufgeteilt und in vertikaler Achse zeitlich versetzt dargestellt werden. Das Signal wird daraufhin über eine CCD-Kamera aufgenommen (siehe Kapitel 3.3.3) und in den Messrechner eingespeist. Zum Schutz der sehr lichtempfindlichen MCP befindet sich hinter der Eingangsoptik noch ein Shutter, welcher im Ruhezustand wie auch bei bestimmten Änderungen im Betriebsmodus den Eingangsbereich automatisch verschließt.

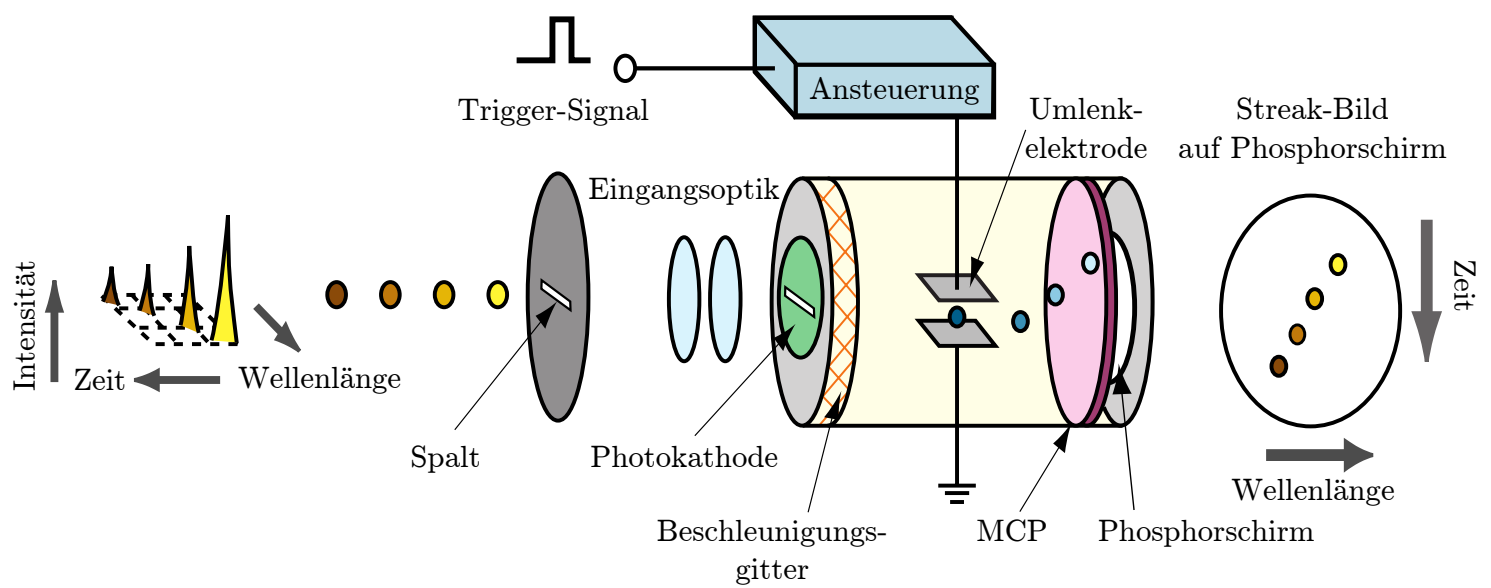

Abbildung 3.23: Schematische Darstellung der Streak-Einheit inklusive Eingangsspalt und Eingangsoptik. Die eintreffenden zeitlich versetzten Lichtpulse unterschiedlicher Wellenlängen lösen von der Photokathode Elektronen aus. Diese werden durch die Vakuumröhre beschleunigt und mit Hilfe einer Umlenkelektrode je nach Eintreffzeitpunkt unterschiedlich abgelenkt. Die Elektronen werden daraufhin durch die MCP vervielfacht und treffen auf einen Phosphorschirm auf, der hierdurch zum Leuchten angeregt wird.

Die zu erreichende zeitliche Auflösung der Streak-Einheit selbst ist an das gewählte Zeitfenster, sowie die Größe des horizontalen Eingangsspalts gebunden. Im Fall von Zeitfenstern einer Länge größer als 2 ns liegt die Halbwertsbreite unterhalb eines Prozents der Fenstergröße (siehe Abbildung 3.25). Für die Bestimmung wurde die dritte Harmonische des Lasers über mehrere Filter abgeschwächt und direkt auf den Eingangsspalt des Spektrometers ausgerichtet. Äquivalent zur Messung der spektralen Auflösung (siehe Kapitel 3.3.1) wurden die resultierenden Verteilungen mit Gleichung 3.9 angepasst. Bei Eingangsspaltbreiten unterhalb von $40 \mu \mathrm{m}$ tritt eine Sättigung mit sogar leichtem Anstieg für Breiten bei $10 \mu \mathrm{m}$ auf. Die beobachtete Sättigung wird durch die endliche Größe der verstärkten Signale am Leuchtschirm als auch durch die Auflösung der aufgenommenen Bilder von der dahinter befindlichen CCD hervorgerufen. Aufgrund der geringen Spaltbreite und dem zusätzlich auch noch verwendeten kohärenten Laserlicht kann es zu leichten Beugungen und 
einer damit einhergehenden Aufweitung hinter dem Spalt kommen, welche durch die Eingangsoptik nicht voll erfasst und somit korrigiert werden kann. Ab Spaltmaßen der Größe $40 \mu \mathrm{m}$ ist ein nahezu linearer Anstieg der Halbwertsbreite von $2.435(10) \cdot 10^{-2} \% / \mu \mathrm{m}$ bei Zeitfenstern größer als $2 \mathrm{~ns}$ zu beobachten. Bei den zwei kleinsten Fenstern hingegen wird die Auflösung durch die experimentelle Anordnung direkt limitiert. In Abbildung 3.25(b) sind beispielhaft zwei Messsignale für das kleinste Zeitfenster dargestellt. Bei $20 \mu \mathrm{m}$ Spaltbreite beträgt hier die Halbwertsbreite 32.3(5) ps. Dies beinhaltet alle Verbreiterungen hervorgerufen durch Jitter (siehe Kapitel 3.1.5) der gesamten Trigger-Anordnung als auch längere zeitliche Drifts über die Aufnahme und Integration von hier 2000 Einzelbildern. Die langsameren Schwankungen wurden jedoch schon durch Kreuzkorrelation (siehe Kapitel 4.3) teilweise kompensiert. In dieser Anordnung befanden sich sehr viele optische Elemente und Spiegel innerhalb des Strahlengangs. Bei einer früheren Messung mit unmittelbarer Einspeisung konnten auch schon Werte von 20 ps erreicht werden. Für eine genauere Darstellung der Messmethodik wird auf das Kapitel 3.3.5 verwiesen, wobei hier die Trigger-Diode in Kombination mit dem Pulspicker Verwendung fand. Abhängig von der Trigger-Frequenz für hohe Wiederholraten zeigen die Zeitfenster $1 \mathrm{~ns}, 2 \mathrm{~ns}$ und $5 \mathrm{~ns}$ bei $1 \mathrm{MHz}$ verglichen zu $4 \mathrm{Mhz}$ schnellere Durchlaufzeiten von jeweils 60(10) ps für das kleinste und 90(10) ps für die beiden anderen. Dies führt bei unterschiedlichen Frequenzen zu einer absoluten leichten Streckung oder auch Stauchung des zeitlichen Signals um maximal 4\%. Verglichen zu einer perfekt linearen Ablenkung beträgt die nichtlineare Abweichung für alle Zeitfenster außer die des 10 ns-Fensters $1 \%$ oder weniger und ist somit für die zu untersuchenden Phänomene zu vernachlässigen. Auch die des $10 \mathrm{~ns}-F e n s t e r s$ liegt mit einem Wert von 1.79(1) \% noch in einem für die hier durchgeführten Messungen nicht relevanten Bereich. Somit wird die zu erreichende Auflösung für die meisten Zeitfenster durch die Größe des horizontalen Eingangsspalts limitiert. Für die praktische Anwendung hat sich eine Größe von $30 \mu \mathrm{m}$ bewährt, da hierdurch zumeist ausreichend Signal auf die Photokathode trifft als auch die zeitliche Aufösung nicht darunter leidet. Des weiteren zeigen sich bei kleineren Spaltöffnungen deutliche Intensitätsschwankungen abhängig von der lateralen Position, bei welcher das Licht auf den Spalt eintrifft. Dies scheint von Unregelmäßigkeiten an der Spaltkante selbst her zu rühren, welche bei solch kleinen Öffnungen stärker zum Tragen kommen.

\subsubsection{ORCA-R2}

Direkt an der Streak-Einheit befestigt befindet sich eine CCD-Kamera (ORCAR2 (C10600-10B), Hamamatsu Photonics K.K.) zur Aufnahme und Übertragung der optischen Signale des Leuchtschirms an dem angeschlossenen Messrechner. Sie besitzt einen Sensor mit $1344 \times 1024$ Pixeln (quadratische Pixel mit $6.45 \mu \mathrm{m}$ Kantenlänge [307]). Zur Reduzierung von thermischen Rauschen befindet sich an der Chiprückseite ein Peltier-Element, welches nach dem gleichnamigen Effekt 308 fun- 


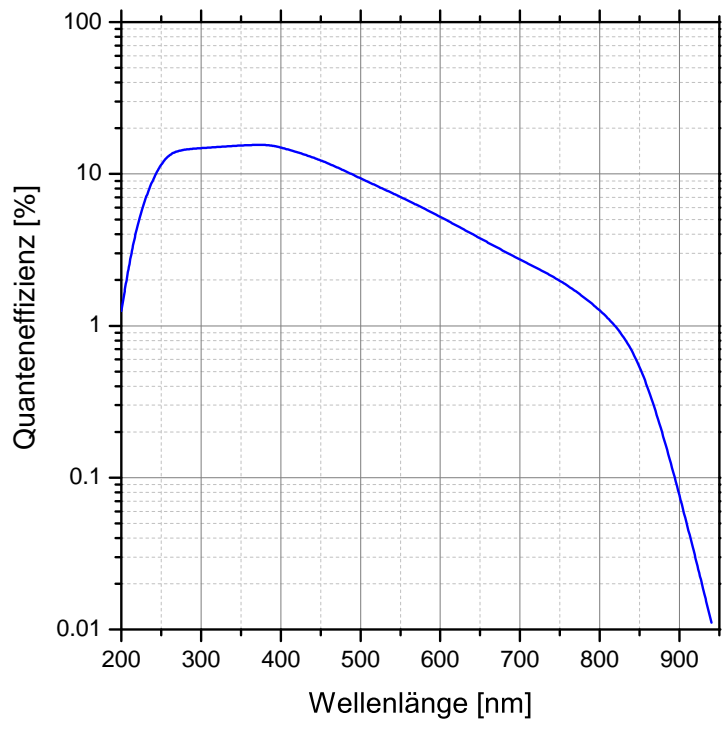

(a)

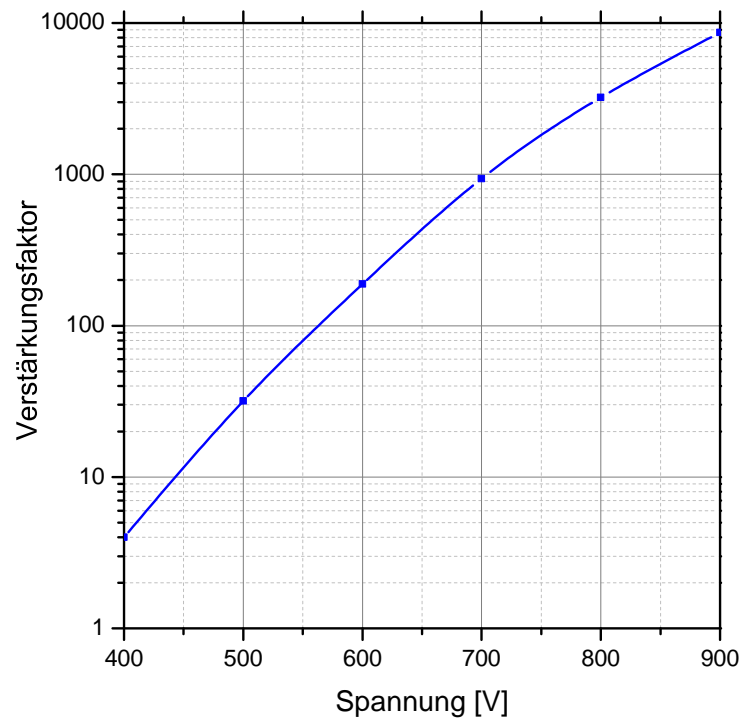

(b)

Abbildung 3.24: (a) Quanteneffizienz der Kathode im relevanten Wellenlängenbereich. Die maximale Quanteneffizienz von $15.8 \%$ wird bei einer Wellenlänge von $380 \mathrm{~nm}$ erreicht. (b) Verstärkungsfaktor der MCP für verschiedene Spannungen. Die Verstärkung kann in der Messsoftware stufenweise eingestellt werden. Die Daten aus beiden Abbildungen entstammen aus dem vor Anlieferung angefertigten Testprotokoll von Hamamatsu Photonics K.K..

gierend für eine Abkühlung auf bis $\mathrm{zu}-40^{\circ} \mathrm{C}$ sorgt. Die dadurch erzeugte Abwärme wird neben einer Luftkühlung durch die schon im Lasersystem eingesetzte Wasserkühlung abgeführt. Laut Hersteller ist auch ein exklusiv luftgekühlter Betrieb möglich. Dieser führt jedoch nach mehreren Stunden Betriebszeit zu einer Erhöhung der Chiptemperatur und einer damit einhergehenden merklichen Steigerung des thermischen Rauschens, was in Langzeitmessungen einen signifikanten Anstieg des Hintergrundsignals hervorrufen kann. Für die durchgeführten Messungen wurde die Kamera im 2×2-Binning-Modus mit einer Aufösung von 12 bit betrieben. Durch das Zusammenfassen von vier Pixeln kann ein schnelleres Auslesen des Chips erfolgen, ohne signifikanten Verlust in der zeitlichen als auch spektralen Aufösung zu erleiden. Alternativ bietet die Kamera auch einen 16 bit-Modus, der etwa zu einer Halbierung der Bildfrequenz führt. Für Photon-Counting-Experimente (siehe Kapitel 3.3.4) ist jedoch eine nicht zu niedrige Bildwiederholfrequenz (hier $28.4 \mathrm{~Hz}$ ) zur Erhöhung der Effizienz bei sehr inhomogenen Intensitäten von Nöten. Eine beispielhafte Aufnahme ohne eigentliches Messsignal ist in Abbildung 3.26 gegeben. Dabei ist ein Untergrundabfall von etwa $10 \%$ von links nach rechts zu beobachten. Dieser rührt vornehmlich nicht von Temperaturdifferenzen auf dem Chip her, sondern scheint 


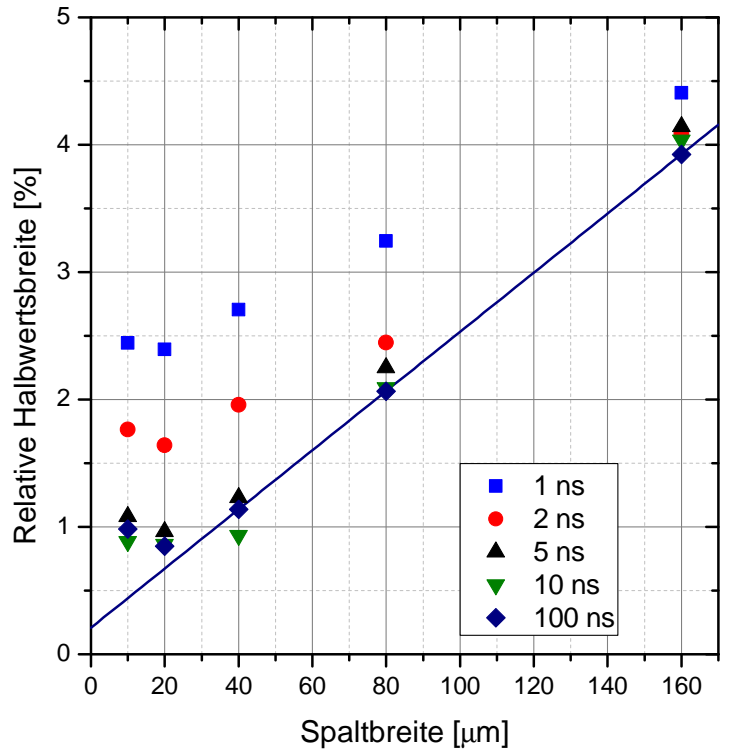

(a)

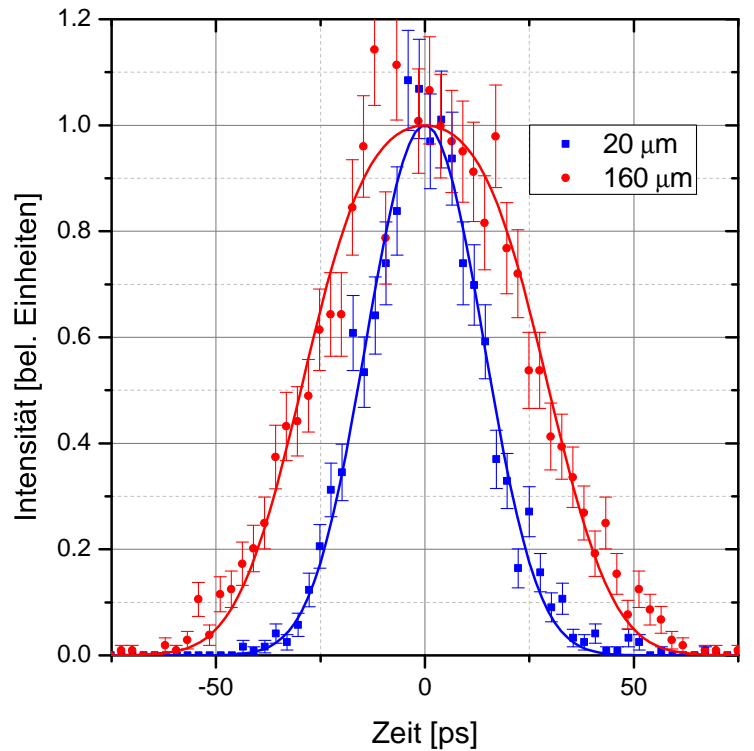

(b)

Abbildung 3.25: (a) Zeitliche Auflösung der Streak-Kamera für unterschiedlich große Zeitfenster. Eingestrahlt wurde mit einer Wellenlänge von $\lambda=267 \mathrm{~nm}$. Die relative Halbwertsbreite bezieht sich hierbei auf die zeitliche Länge des gewählten Fensters. Bei der blauen Linie handelt es sich um eine lineare Anpassung für ein Zeitfenster mit Spaltbreiten größer als $40 \mu \mathrm{m}$. (b) Signal bei kleinstem Zeitfenster (1 ns) für zwei verschiedene Spaltbreiten. Die Fits erfolgten nach Gleichung 3.9.

an die Auslesemethode gebunden zu sein, da sich im 16 bit-Modus das Untergrundverhältnis umkehrt. Der Untergrund verhält sich dabei bis zu Belichtungszeiten von über 1s relativ konstant und entspricht einem Wert von etwa 2.5\% der Sättigung, welche im 12 bit-Modus bei 4095 Zählern erreicht ist. Ein Ausschnitt eines typischen Messbildes ist in Abbildung 3.27 dargestellt. Auch hier bleiben die Intensitäten zumeist im einstelligen prozentualen Bereich verglichen zur vollen Sättigung des Chips. Bei zu hohen Intensitäten besteht die Gefahr einer Beschädigung der entsprechenden Bereiche der MCP in der Vakuumröhre der Streak-Einheit, was zur Erblindung dieser führen kann. Weiterhin führen hohe Verstärkungsfaktoren zu einem starken Anwachsen der Signalbreite, was je nach Messmodus zu niedrigeren Zählraten oder fehlerhaften Zuordnungen von Ereignissen führen kann. Aufgrund der vor der CCD-Kamera angebrachten Ausgangsoptik der Streak-Röhre und der Geometrie des Phosphorschirms zeigen sich über den gesamten Messbereich Inhomogenitäten bezüglich der Sensitivität auf ein eigentlich homogenes Eingangssignal (siehe Abbildung 3.28). Insbesondere auf der rechten Seite und zu allen Bildecken hin ist hierbei ein starker Sensitivitätseinbruch zu beobachten. In der Mitte als auch 
in Richtung der linken Seite betragen die Abweichungen zumeist nur etwa 10 - 20\%, welche durch geeignete Korrekturen (siehe Kapitel 4.2) ausgeglichen werden können.

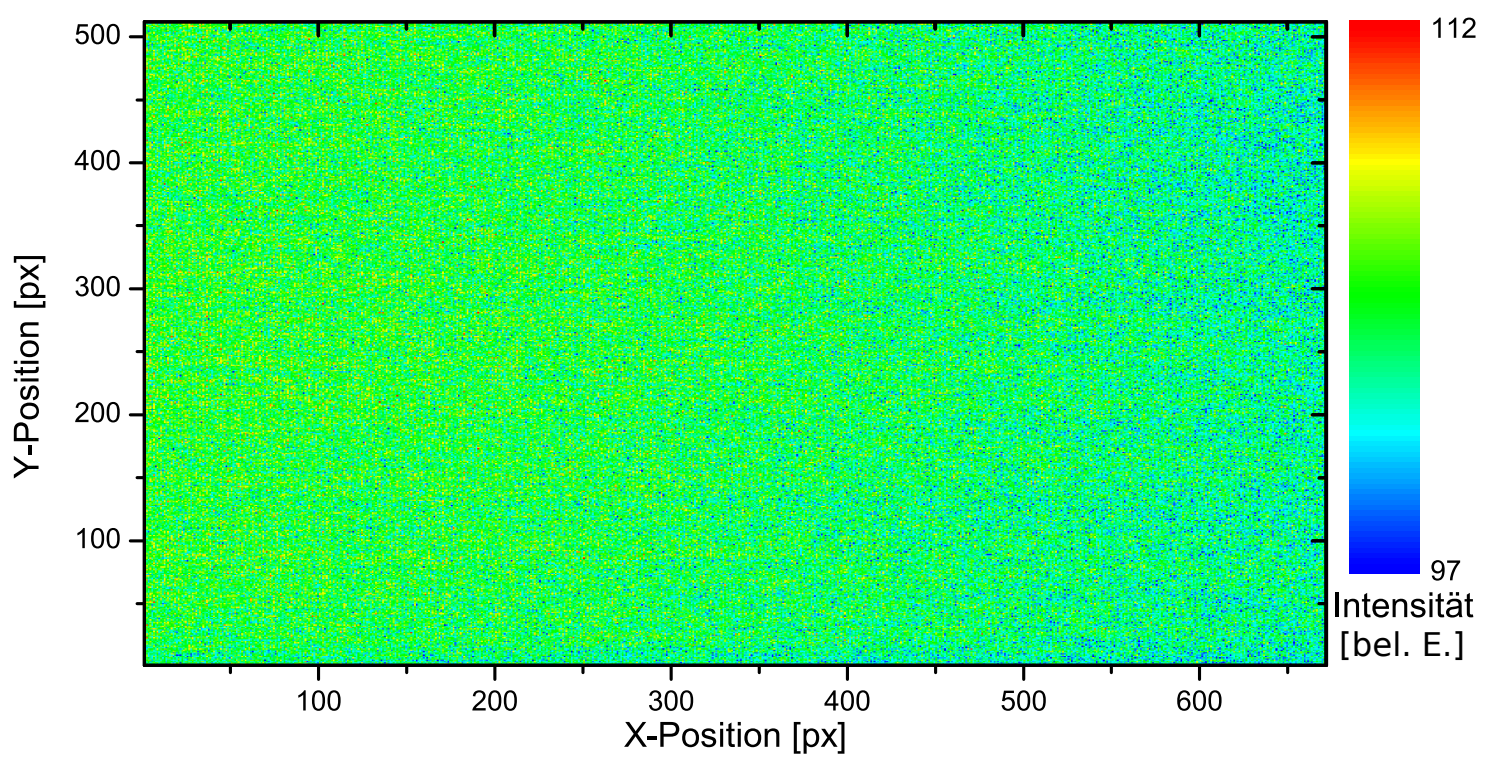

Abbildung 3.26: Aufnahme eines Bilds mit $100 \mathrm{~ms}$ Belichtungszeit bei geschlossenem Shutter, 2×2-Binning und möglichst niedriger MCP-Verstärkung (0 in Steuersoftware). Zur linken Seite zeigt sich ein leicht erhöhter Untergrund hervorgerufen hauptsächlich durch die verwendete schnelle Auslesemethode.

\subsubsection{Messverfahren}

Die Messsoftware zur Steuerung der Streak-Kamera bietet zwei Messmodi mit grundsätzlich sehr unterschiedlicher Funktionalität. Dabei handelt es sich zum einen um eine analoge Integration der einzelnen Messbilder oder als Alternative um einen eher digitalen Ansatz über den Einzelphotonen-Ereignisse registriert werden können. Letzterer kam bei nahezu allen Messungen, die im Laufe der Arbeit durchgeführt wurden, zum Einsatz. Beide werden nun im Folgenden kurz dargestellt.

\section{Photon-Counting}

Beim Photon-Counting, das hauptsächlich zum Einsatz kam, handelt es sich um ein Verfahren, bei dem Ereignisse nur als solche gewertet werden, wenn sie einen frei einstellbaren Schwellwert überschreiten. Dieser kann über eine automatische Hintergrundmessung bei geschlossenem Shutter an der Streak-Kamera bestimmt werden, fällt jedoch häufig mit Werten um 115 sehr hoch aus. Alternativ kann die Zuordnung auch manuell erfolgen. Als praktikabel hat sich dabei ein Wert zwischen 


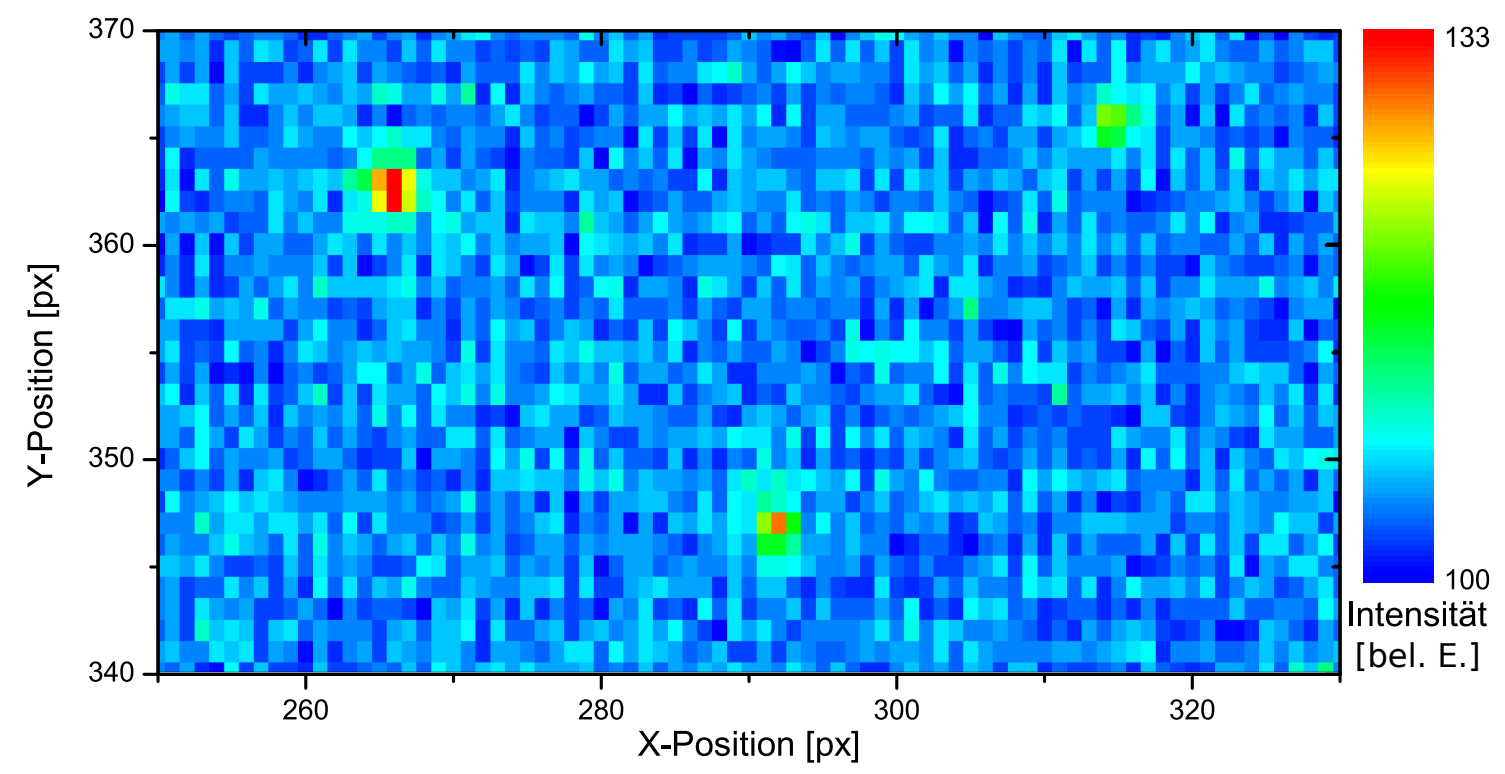

Abbildung 3.27: Ausschnitt eines Bilds mit $100 \mathrm{~ms}$ Belichtungszeit bei geschlossenem Shutter, $2 \times 2$-Binning und einer mittleren MCP-Verstärkung (27 in Steuersoftware). Die Sweep-Dauer beträgt $1 \mathrm{~ms}$. Bei den Signalen handelt es sich um Untergrundereignisse.

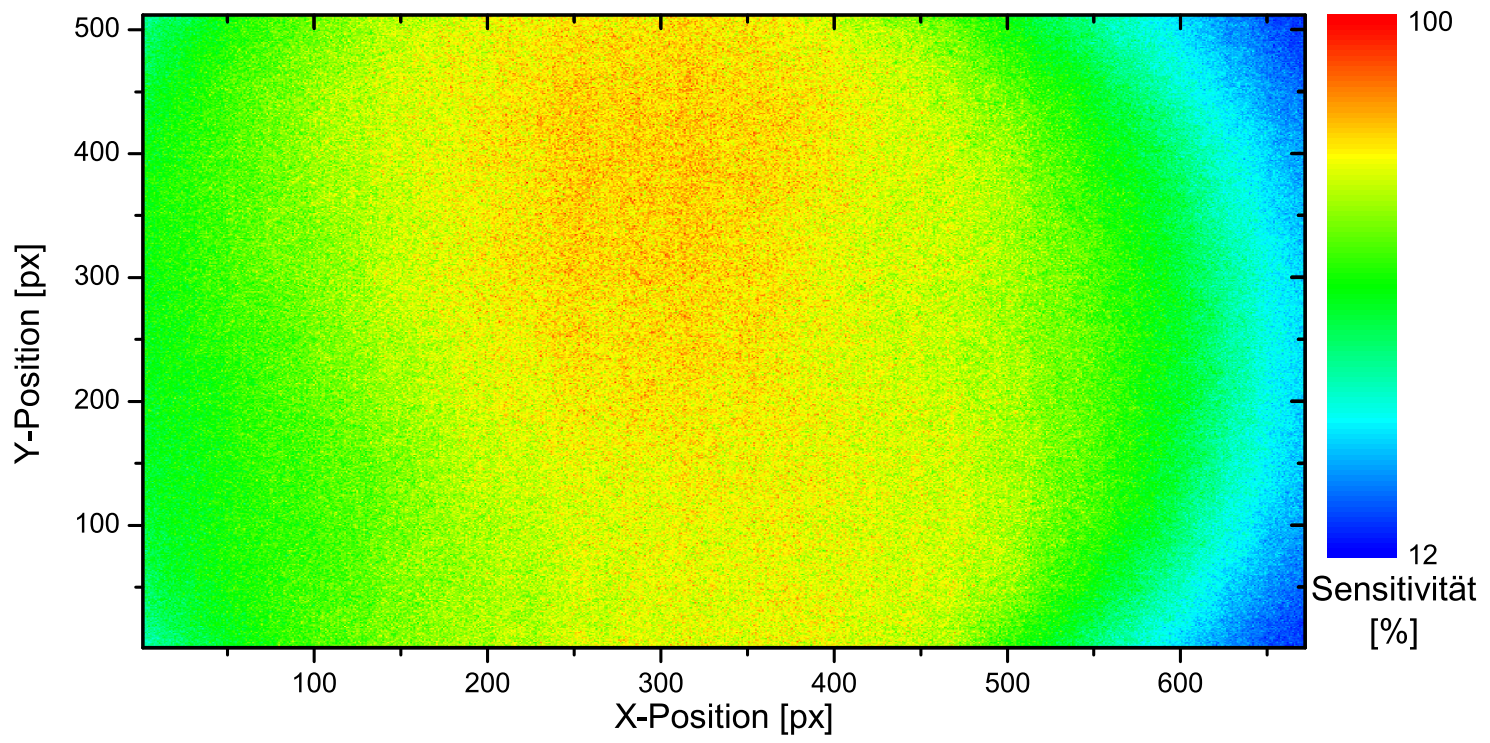

Abbildung 3.28: Sensitivitätsverteilung auf dem CCD-Chip. Aufgenommen wurde ein breitbandiges Lumineszenzsignal mit dem 900er Gitter im Photon-CountingModus ohne Synchronisation mit dem Laser. Zur rechten Seite und vor allem zu den Bildecken zeigt sich ein starker Sensitivitätsabfall. 
98 und 100 erwiesen. Bei niedrigeren Werten steigt das Hintergrundsignal signifikant an, während bei höheren Werten eine deutlich erhöhte Verstärkung für die Messung von Signalen von Nöten ist. Insbesondere bei inhomogenen Intensitätsverteilungen kann dies zu hohen Belastungen der MCP führen. Überschreiten ein oder mehrere Pixel in ihrer Intensität den Schwellwert, wird aus diesen im Fall von benachbarten Pixeln ein Schwerpunkt gebildet und jener als ein Ereignis gewertet (siehe Abbildung 3.29). Aufgezeichnet werden diese Signale in einem einfachen zweidimensionalen Histogramm, indem jedes Ereignis den Zählwert an der entsprechenden Stelle um eins erhöht. Mit einer Bildfrequenz der CCD-Kamera von typischerweise $28.4 \mathrm{~Hz}$ im $2 \times 2$-Binning-Modus baut sich so nach und nach ein zeitaufgelöstes Messsignal auf. Beispielhaft ist solch eine Messung in Abbildung 3.30 dargestellt. Neben dem Histogramm lässt sich jedoch auch jedes einzelne Signal mit einem Zeitstempel versehen, wodurch eine nachträgliche Analyse und auch Bearbeitung ermöglicht wird. Dies kann beispielsweise zur Korrektur von zeitlichen Drifts über eine längere Messzeit hinweg genutzt werden (siehe Kapitel 4.3).

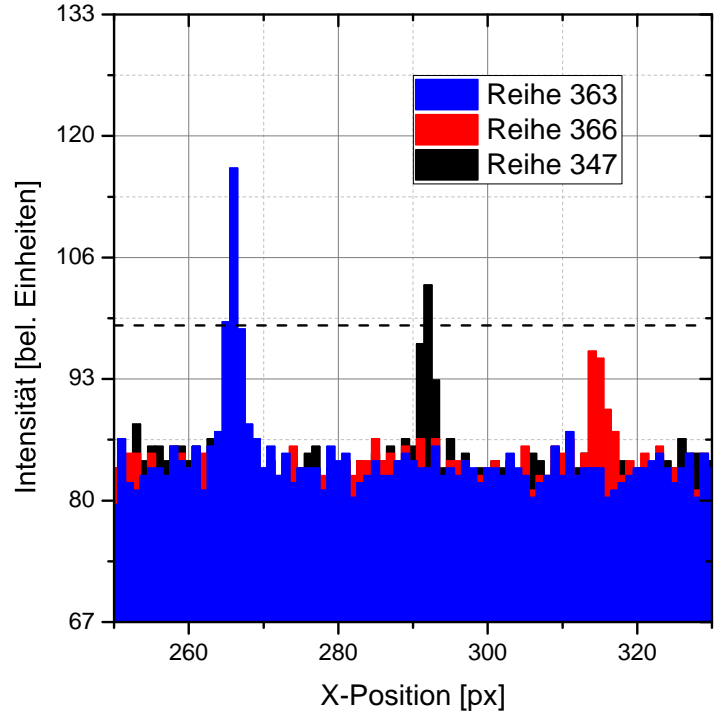

Abbildung 3.29: Ausschnitt von drei Pixelreihen der Abbildung 3.27. Der eingezeichnete Schwellwert von 99 führt zu einer Zählung des blauen als auch des schwarzen Signals. Im Fall des blauen Signals wird der Schwerpunkt der benachbarten Pixel gebildet. Das rote Signal hingegen findet bei der Zählung keinerlei Beachtung. Der konstante Untergrund bleibt ebenfalls unberücksichtigt und taucht dahingehend in den Messergebnissen nicht auf.

\section{Analoger-Modus}

In diesem Modus wird der Intensitätswert jedes Pixels unabhängig von dessen Höhe Bild für Bild in einem 2D-Histogramm aufintegriert. Je nach Verstärkungsfaktor der MCP können dabei unterschiedlich große "Kegel" um ein jeweiliges Zentrum entstehen, was zu einer niedrigeren spektralen als auch zeitlichen Auflösung bei der Integration von vielen aufeinander folgenden Bildern führen kann. Jedoch bietet diese Methode ein gutes Mittel für Messungen bei geringen Repititionsraten oder auch nur Einzelschuss-Messungen mit nur einem Bild, bei denen aber die Intensitäten entsprechend höher ausfallen. Ein Beispiel für eine integrierte analoge 


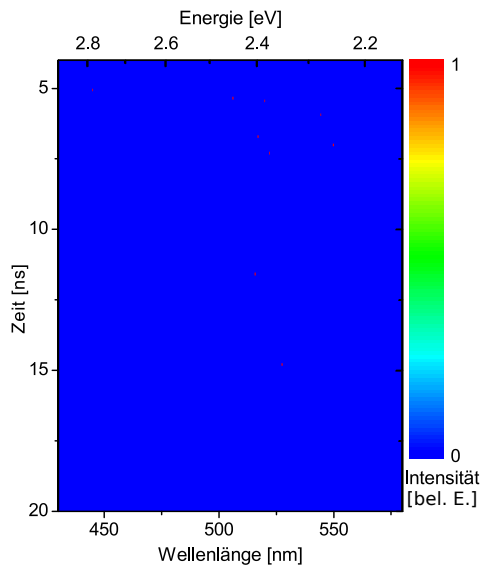

(a) 1 Bild

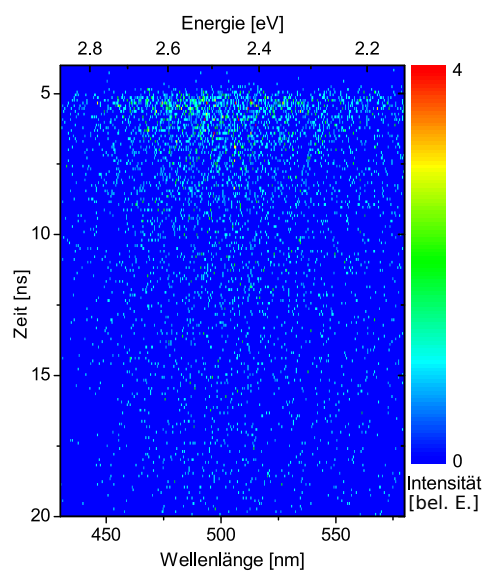

(d) 1000 Bilder

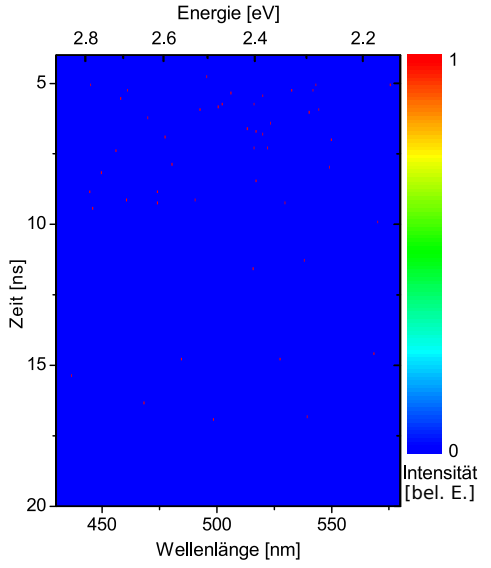

(b) 10 Bilder

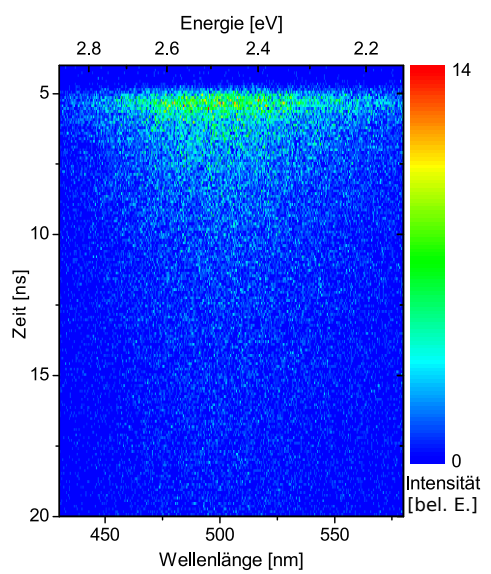

(e) 10000 Bilder

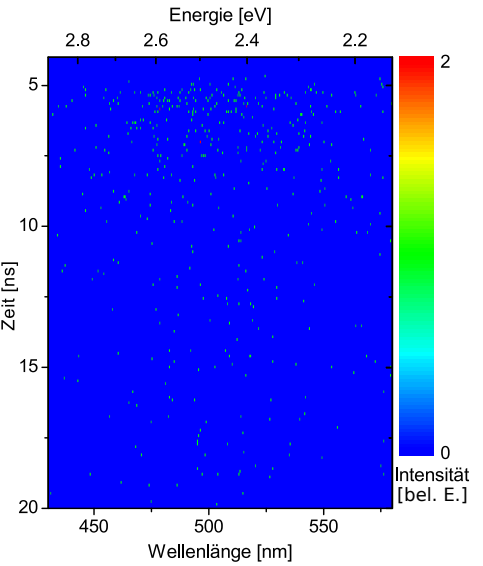

(c) 100 Bilder

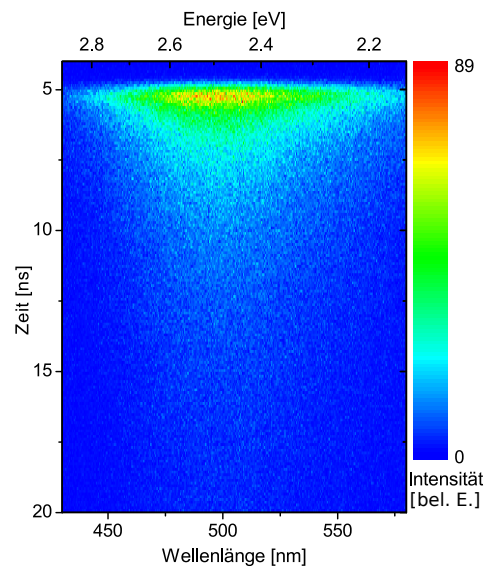

(f) 100000 Bilder

Abbildung 3.30: Aufnahmen einer Defektlumineszenz in $\mathrm{TiO}_{2}$. Die einzelnen Abbildung zeigen den Fortschritt einer Messung im Photon-Counting-Modus. Die Anzahl der zu jedem Zeitpunkt integrierten Bilder ist jeweils unten angegeben. Dabei entsprechen 100000 Bilder einer Messzeit von etwa einer Stunde. 
Messung ist in Abbildung 3.31 dargestellt. Durch die direkte Integration lässt sich in diesem Modus eine nachträgliche Korrektur durch zeitliche Drifts nur schwer durchführen. Jedoch bietet die Messsoftware zusätzlich auch eine Speicherung jedes einzelnen Bildes an, was aber bei Langzeitmessungen einen deutlich erhöhten Speicherplatzbedarf verursacht. Die Belichtungszeit im analogen Modus kann verglichen zum Photon-Counting allerdings deutlich länger gewählt werden (z. B. $100 \mathrm{~ms}$ ), was eine niedrigere Bildanzahl pro Messung zur Folge hat. Weiterhin findet hier standardmäßig der 16 bit-Modus der CCD-Kamera (siehe Kapitel 3.3.3 Anwendung, wodurch Intensitätsunterschiede deutlich besser aufgelöst werden können; insbesondere da sich die Einzelbilder typischerweise weit unterhalb der Sättigung der CCD befinden.

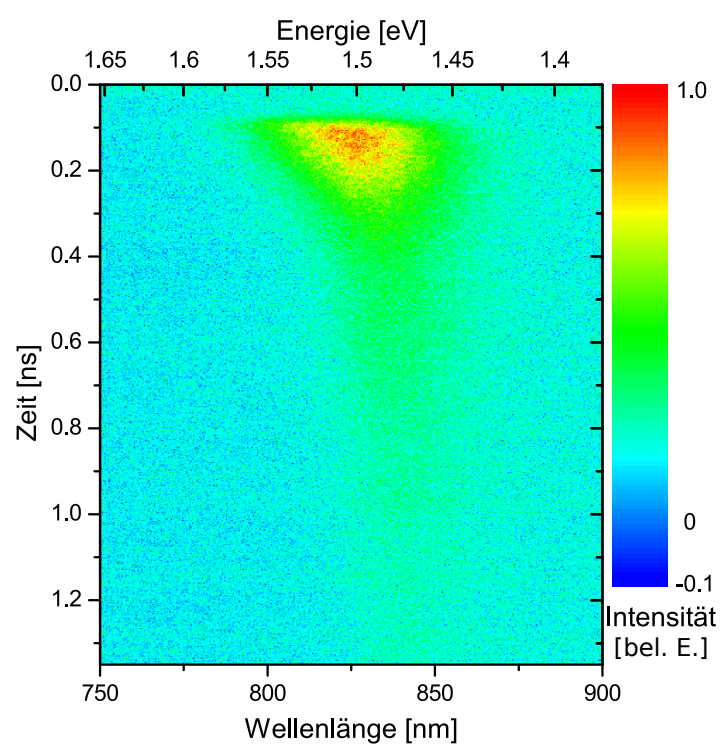

Abbildung 3.31: Aufnahme einer bandkantennahen Lumineszenz in GaAs im analogen Modus. Die Aufnahmezeit beträgt etwa 30 Minuten. Das von der CCD erzeugte Hintergrundsignal wurde durch eine Subtraktion einer Messung bei geschlossenem Shutter, welche ebenfalls 30 Muniten andauerte, kompensiert. Hierdurch zeigt sich jedoch ein starkes statistisches Rauschen, welches für jeden einzelnen Pixel etwa $10 \%$ der höchsten Intensität im Bild entspricht.

\subsubsection{Arbeitsmodi}

Das Auslösen eines Schusses der Streak-Kamera kann je nach Anwendung über unterschiedliche Trigger-Quellen erfolgen. Die in diesem Versuchsaufbau zur Verfügung stehenden und auch im Rahmen dieser Arbeit teilweise speziell hierfür konstruierten Varianten werden im folgenden Abschnitt kurz erläutert. Zentrale Komponente zur Erzeugung des finalen Trigger-Pulses für die Streak-Einheit ist in allen Fällen der mit dem System ausgelieferte taktgesteuerte Pulsgenerator (C10647-01, Hamamatsu Photonics K.K.). Dieser beinhaltet zusätzlich eine Verzögerungseinheit einstellbar vom Sekundenbereich bis hin zu einer Granularität im Bereich von Pikosekunden [309]. Als Ausgangsanschlüsse besitzt die Einheit vier BNC-Buchsen, von welchen die jeweilige Verzögerungszeit sowie auch die Pulsbreite separat eingestellt werden können. 


\section{Interner Modus}

In diesem Modus wird kein externes Trigger-Signal benötigt. Hier übernimmt der C10647-01 selbst die Erzeugung von Trigger-Ereignissen, welche beispielsweise zur Ansteuerung externer gepulster Signalquellen genutzt werden können. Der Frequenzbereich erstreckt sich dabei von mindestens $20 \mathrm{mHz}$ bis zu maximal $20 \mathrm{MHz}$ [309]. Neben der Ansteuerung externer Quellen eignet sich dieser Modus auch zum gezielt asynchronen Betrieb mit dem weiter oben vorgestellten Lasersystem (siehe Kapitel 3.1), wodurch beispielsweise Ausleuchtungstests (siehe Abbildung 3.28) des Phosphorschirms und der an der Streak-Kamera angebrachten CCD durchgeführt wurden.

\section{Photodiode und Pulspicker}

Für Messungen von Lebensdauern deutlich kürzer als $100 \mu$ s kommt eine Photodiode (DET10A/M, Thorlabs Inc.) zur Erzeugung des Trigger-Pulses zum Einsatz. Hierfür wird ein Teil der Laserleistung direkt hinter dem Laserkopf des Titan-SaphirLasers (siehe Kapitel 3.1.2 mit Hilfe einer Rasierklinge abgezweigt und ggf. über eine Sammellinse auf die Photodiode fokussiert. Versuche durch Beam-Splitting, beispielsweise mit einer dünnen Deckglasscheibe, erwiesen sich als weniger effizient, da hierdurch ein unnötig großer Teil der Laserleistung abgezweigt wurde. Die Diode wird in Sperrrichtung betrieben, wobei eine auswechselbare Batterie die dafür nötige Spannung zur Verfügung stellt. Je nach einfallender Laserleistung muss diese regelmäßig (spätestens nach 24 Stunden Messzeit) ersetzt werden, da eine Reduzierung der Gegenspannung auch die Stärke des Ausgangssignals mindert. Eine kleine Änderung in der Höhe jenes führt aufgrund einer schwellwertbasierten Erzeugung des finalen Triggers innerhalb des C10647-01 zu einer Verschiebung des zeitlichen Nullpunkts und damit zu einem Drift des Messsignals. Dieser Effekt ist ebenfalls bei der Erwärmung des Systems im Betrieb über längere Zeit und einer damit einhergehenden Änderung der Laserleistung zu beobachten, lässt sich jedoch in der Regel im Fall von Photon-Counting-Messungen noch im Nachhinein minimieren (siehe dazu Kapitel 4.3). Die Diode besitzt einen nutzbaren Sensitivitätsbereich von $200-1100 \mathrm{~nm}$ und eine Anstiegszeit von etwa $1 \mathrm{~ns}$ [310], was den Justagebereich des Lasers somit voll abdeckt. Innerhalb des Laserkopfes befindet sich zwar ebenfalls eine Photodiode, welche ein mit den Ausgangspulsen synchronisiertes Signal generiert, allerdings wird jene zur Ansteuerung des Pulspickers (siehe Kapitel 3.1.3) verwendet. Dieser liefert dazu ein synchrones Ausgangssignal, dessen Jitter jedoch etwa 100 ps beträgt, was dementsprechend bei Messungen mit kleinen Zeitfenstern auch zu einer Reduzierung der Auflösung führen würde.

In Kombination mit dem Pulspicker erfolgt die Trigger-Erzeugung im C10647-01 im sogenanntem Dump-Modus. Hierbei generiert der Pulspicker ein Referenzsignal, welches einen relativ hohen zeitlichen Jitter aufweisen kann. Nach der im C10647-01 
einstellbaren Verzögerungszeit öffnet dieser ein Tor und erzeugt mit dem nächsten eintreffenden Signal der Photodiode ein Ausgangssignal mit niedrigem zeitlichen Jitter. Somit lassen sich, da das Signal mit den Laserpulsen synchronisiert ist, einzelne Pulse direkt auswählen. Dadurch ist jedoch eine genaue zeitliche Positionierung nicht möglich; lediglich eine diskrete Verschiebung, vorgegeben durch die Laserfrequenz (76 MHz), um 13.2 ns-Schritte. Eine präzisere Justage wird durch das Nachschalten einer weiteren Verzögerungseinheit (C1097-05, Hamamatsu Photonics K.K.), welche Zeiten zwischen 0-31.96 ns liefert [311], ermöglicht. Der zeitliche Ablauf für die Erzeugung eines einzelnen Trigger-Pulses bis zum Schuss der Streak-Kamera ist in Abbildung 3.32 noch einmal grafisch zusammengefasst.

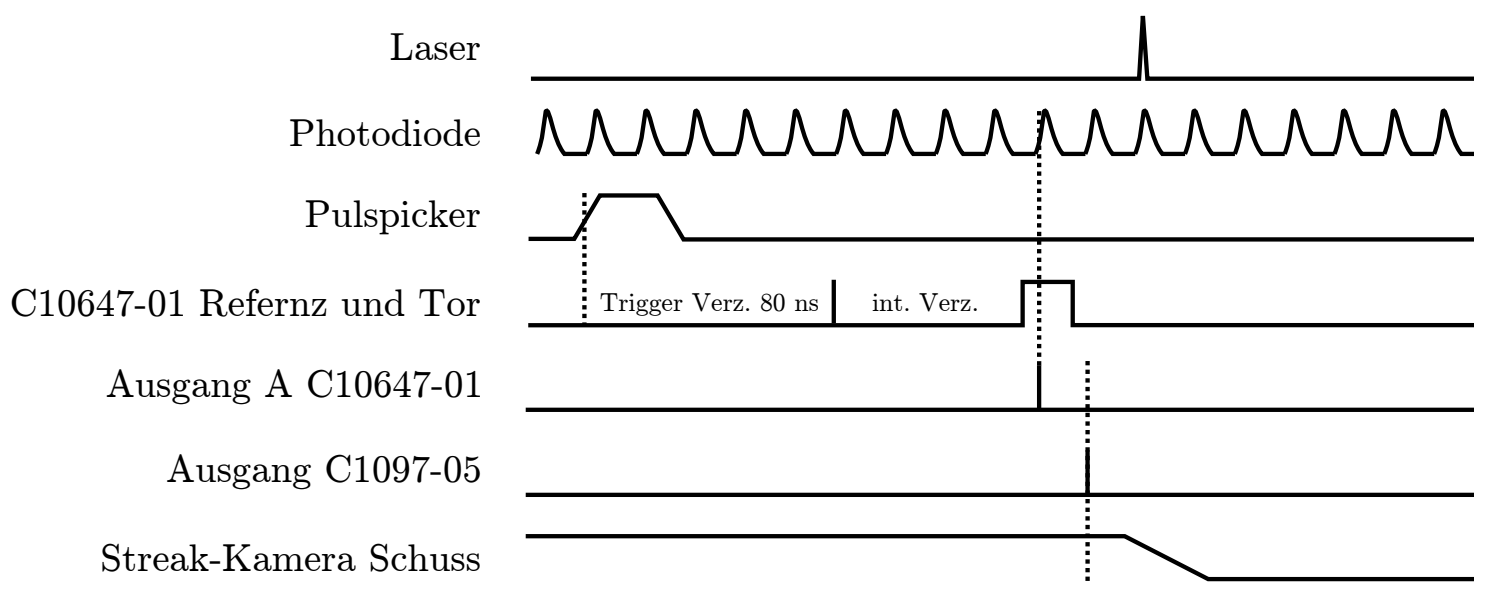

Abbildung 3.32: Signalverlauf zum Triggern kurzlebiger Ereignisse. Das Signal des Pulspickers erzeugt im C10647-01 eine interne Referenz nach etwa 80 ns [309]. Darauf folgt eine frei wählbare Verzögerung an die anschließend ein internes Tor geöffnet wird. Der nächste von der Photodiode eingehende Puls erzeugt ein Trigger-Signal, welches mit dem C1097-05 weiter verzögert und zur Synchronisation zwischen Laser und Streak-Kamera gebracht wird.

\section{Chopperrad und Trigger-Generator}

Für Lumineszenzen mit Lebensdauern größer als $100 \mu$ s kommt das schon in Kapitel 3.1.5 beschriebene Chopperrad zur Generierung eines Trigger-Signals zum Einsatz. Dieses kann, da auch hier der Laser zur Erzeugung von Harmonischen im Pulsbetrieb operiert, als Taktgeber an Stelle des Pulspickers treten. Somit würde der C1064701 wieder im Dump-Modus und die Signalkette äquivalent zu Abbildung 3.32 verlaufen. Da der zeitliche Jitter in diesem Fall jedoch hauptsächlich aus einer mechanischen Unwucht des Rads selbst resultiert, kann auch ein Betrieb des C1064701 im sogenannten externen Modus erfolgen. Hierbei werden je nach Einstellung die 
steigenden oder fallenden Flanken aus dem Controller des Chopperrads aufgenommen und daraus Trigger-Pulse generiert [309].

Da das größte Zeitfenster der Streak-Kamera nur eine Länge von $1 \mathrm{~ms}$ (siehe Kapitel 3.3.2 besitzt, lassen sich langsamere Prozesse mit Lebensdauern im hohen Mikrosekunden- oder sogar Millisekunden-Bereich auf diese Weise nur schwerlich untersuchen. Zwar können bei Zeitskalen dieser Größenordnung auch auf andere Funktionsweisen basierende Systeme eingesetzt werden, jedoch erschien eine mögliche Ausweitung des Aufnahmebereichs zu größeren Zeiten hin eine sinnvolle Erweiterung der Funktionalität des in diesem Kapitel vorgestellten experimentellen Aufbaus. Hierfür wurde ein Pulsgenerator mit variabler Verzögerungszeit und Pulszahl entwickelt. Die Grundlage bildet dabei ein Entwicklerboard (STM32 F4 Discovery, STMicroelectronics N.V.) mit ARM Cortex-M4 Prozessor, welcher eine Taktgeschwindigkeit von bis zu $168 \mathrm{MHz}$ [312] besitzt. Die Steuerung der Karte erfolgt über USB und eine eigens für diese Anwendung entwickelte Steuersoftware, welche sowohl auf Windows als auch auf aktuellen Linuxdistributionen lauffähig ist. Eine Erweiterung des nutzbaren Zeitfensters gelingt im vorliegenden Fall über eine sequentielle Verschiebung bezüglich des eigentlichen Trigger-Signals. Hierfür wird die Trigger-Einheit hinter den C10647-01 geschaltet. Das Board generiert ein Trigger-Signal, welches für eine frei wählbare Dauer eine bestimmte Verzögerung zum Ursprungssignal aufweist. Danach tritt eine kurze Pause (auch frei wählbar) ein. Mit Hilfe dieser lassen sich die späteren Zeitfenster voneinander trennen. Daraufhin erzeugt die Einheit ein um die Größe eines Zeitfensters verschobenes Signal mit vergleichbarer Dauer zu der des vorherigen. Dies wird für die gewünschte Anzahl der zu einander verschobenen Fenster wiederholt. Nach der letzten Verschiebung springt der Generator zur ersten Verzögerungszeit zurück und der Vorgang beginnt von vorn. Die Aufnahmedauer pro Fenster liegt je nach deren Anzahl bestenfalls im Bereich von einigen Sekunden, da somit langsame Änderungen in der Intensität beispielsweise hervorgerufen durch Variationen der Laserleistung oder Veränderungen auf der Probe bestmöglich über die Mittlung von vielen Zyklen kompensiert werden können. Eine schematische Darstellung zur besseren Verdeutlichung der Funktionsweise ist in Abbildung 3.33 gegeben. Diese Aufnahmetechnik lässt sich exklusiv nur im Photon-Counting-Modus in Kombination mit einer sequentiellen Speicherung der Daten nutzen, da die zeitlichen Informationen der Ereignisse zur Zuordnung zum jeweiligen Zeitfenster benötigt werden. Die spätere Rekonstruktion zu einem Messbild erfolgt in einer im Rahmen dieser Arbeit speziell für dieses Streak-System entwickelten Auswerte-Software, auf welche in Kapitel 4 genauer eingegangen wird. 


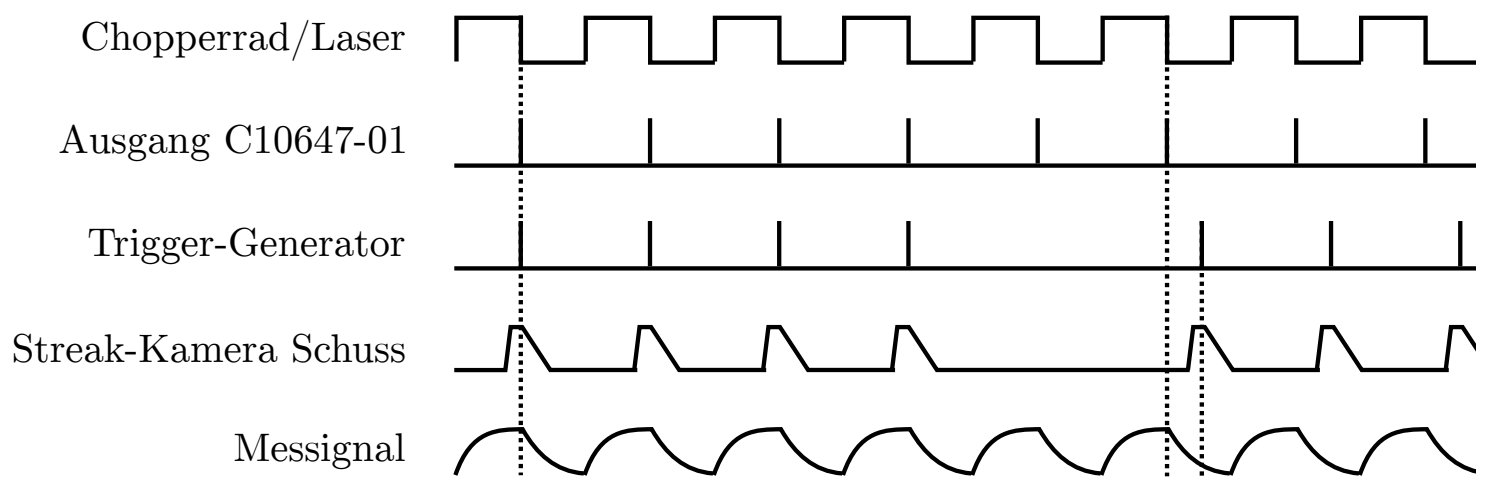

Abbildung 3.33: Signalverlauf zum Triggern sehr langlebiger Ereignisse. Der C10647-01 generiert aus den Rechteckpulsen des Chopper-Controllers ein primäres Trigger-Signal. Dieses wird vom darauf folgendem Trigger-Generator an die StreakEinheit weitergeleitet. Dabei wird nach einem Pulszyklus eine kurze Pause erzeugt und anschließend mit einer größeren Verzögerung ein neuer Zyklus gestartet. 


\section{Datenanalyse}

Für eine effektive Auswertung der mit dem zeitaufgelösten Lumineszenzaufbau aufgenommenen Messreihen wurde im Rahmen dieser Arbeit eine Software basierend auf dem Qt-Framework programmiert. Diese ist in der Lage, die verschiedenen proprietären Datenformate der Messsoftware HPDTA 9.2 (Hamamatsu Photonics K.K.), die zur Steuerung der Streak-Einheit und des Spektrometers zum Einsatz kommt, nativ weiter zu verarbeiten. Im folgenden Kapitel wird auf die verschiedenen Methoden und Möglichkeiten der Datenanalyse und Fehlerminimierung diesbezüglich eingegangen.

\subsection{Untergrundsignal}

Das während einer Messung aufgenommene Untergrundsignal hängt im wesentlichen vom gewählten Schwellwert (siehe Kapitel 3.3.4), dem Verstärkungsfaktor in der MCP (siehe Kapitel 3.3.2) und dem betrachteten Zeitfenster ab. Des weiteren bestimmt sich das Verhältnis zwischen Untergrund und Messsignal durch die Repetitionsrate der während der Messung durchgeführten Sweeps der StreakKamera. Diese wird maßgeblich durch die von außen angelegte Trigger-Frequenz beeinflusst, wodurch je nach Konfiguration vor allem bei kurzen Zeitfenstern sehr unterschiedliche maximale Schussraten erreicht werden können. Die maximal gemessenen Geschwindigkeiten sind in Abbildung 4.1 dargestellt. Für die drei kleinsten Zeitfenster gilt demnach die vom Hersteller angegebene höchste Grenze der SlowSweep-Unit von $4 \mathrm{MHz}$ [303]. Danach nimmt die Geschwindigkeit sprunghaft ab und verringert sich daraufhin kontinuierlich; auch bedingt durch die endliche zeitliche Länge der Zeitfenster. Mit deren Größe hingegen wächst die während einer Messung aufgenommene Zahl von Hintergrundereignissen kontinuierlich an (siehe Abbildung 4.1) und skaliert nahezu linear mit der reellen Aufnahmedauer. Jene beträgt beispielsweise im kleinsten Zeitfenster (1.35 ns) maximal nur etwa $5.4 \mathrm{~ms} / \mathrm{s}$ im Vergleich zum längsten Fenster (1.01 ms) mit etwa $479 \mathrm{~ms} / \mathrm{s}$. Aufgrund der geringeren Repititionsrate bei langlebigeren Prozessen sinkt zum einen die eigentliche Signalintensität (zumindest im Fall der Nutzung des Pulspickers) und zum anderen steigt der gemessene Hintergrund durch eine real längere Aufnahmezeit pro Intervall. Somit ergibt sich ein verschlechtertes Signal-zu-Rauschverhältnis mit zunehmender Fenstergröße. Die Verteilung des Hintergrundsignals folgt zu Teilen der örtlichen Sensitivität der Streak-Einheit (siehe Kapitel 3.3.3). Die in Abbildung 
4.1 angegebenen Hintergrundereignisse pro Streak-Kameraschuss wurden bei einem Schwellwert von 100 aufgenommen (siehe Kapitel 3.3.4). Je nach Temperatur des Kamerachips steigt bei niedrigeren Werten unabhängig vom gewählten Zeitfenster die Zahl von "Geisterereignissen" signifikant an, da so teilweise auch Fluktuationen im kontinuierlichen Untergrund jenen Schwellwert überschreiten können und somit als Einzelereignis registriert werden.

Eine Hintergrundmessung kann über eine Aufnahme mit geschlossenem Shutter oder besser bei identischer Konfiguration nur mit blockierter Anregung erfolgen. Eine komplette Deaktivierung ist zumeist nicht möglich, da bei einer Anregung mit Photodiode und Pulspicker zur Auslösung eines Kameraschusses das eingeschwungene Lasersystem benötigt wird (siehe Kapitel 3.3.5). Aus solch einer Messung kann mittels der Auswertesoftware eine zweidimensionale Hintergrunddatei erstellt werden, welche ihrerseits auf die Aufnahmedauer normiert ist und somit automatisiert für Messungen mit unterschiedlichen Messzeiten zur Verfügung steht. Bei einer Subtraktion einer Messdatei werden die resultierenden statistischen Unsicherheiten in den Fehlern der Messdaten berücksichtigt.

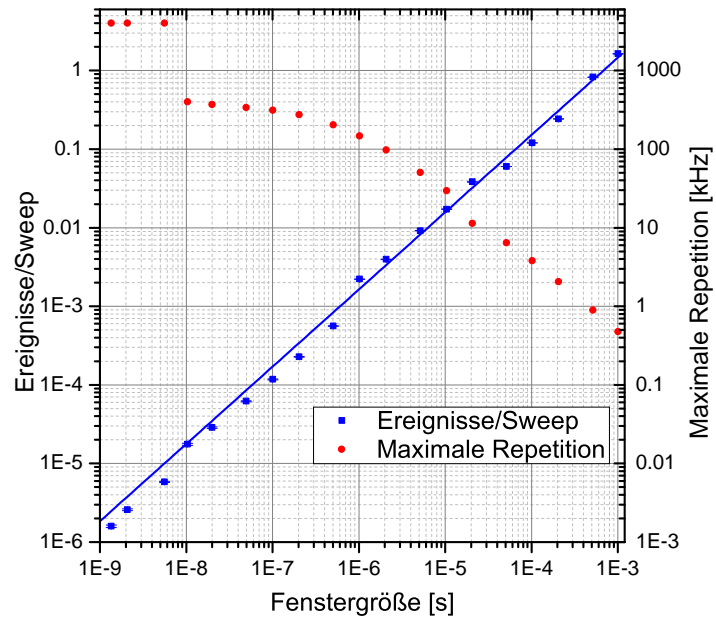

Abbildung 4.1: Hintergrundsignal pro Sweep (blau) und die maximal mögliche Repetition der Streak-Kamera (rot) für die verschiedenen Zeitfenster. Die Zahl der Hintergrundereignisse (gemessen bei geschlossenem Shutter) bezieht sich auf den kompletten Fensterbereich und skaliert nahezu linear mit der Aufnahmedauer. Bis auf für die kleinsten Zeitfenster (Repetition von $4 \mathrm{MHz}$ ) reduziert sich die Schussgeschwindigkeit der StreakKamera kontinuierlich mit deren Länge (Anregung gemessen mit $20 \mathrm{MHz}$ ).

\subsection{Sensitivität}

Wie schon in Kapitel 3.3.3 angedeutet, zeigt sich verteilt über den CCD-Chip der ORCA-R2 eine inhomogene Sensitivitätsverteilung, welche zum einen durch die Auslesemethodik und zum anderen durch die vor der Kamera befindliche Optik und eine damit ungleichmäßige Ausleuchtung hervorgerufen wird. Im Fall von zeitaufgelösten Messungen führt dies dazu, dass nicht der eigentlichen Realität entsprechende Transienten beobachtet werden (siehe Abbildung 4.2(a)). Besonders auffällig ist jener Umstand in Messungen bestehend aus mehreren Zeitfenstern (siehe Kapitel 
3.3.5), wie in Abbildung 4.2(b) dargestellt. Neben dieser in zeitlichen Messungen auftretenden Diskrepanzen existiert auch über die verschiedenen Wellenlängen eine stark variierende Sensitivitätsverteilung des Messaufbaus, hervorgerufen durch das jeweilig verwendete Gitter, die Spiegel innerhalb des Spektrometers, die Eingangsoptik der Streak-Kamera, die in der Kamera befindliche Photokathode sowie die eigentliche Einstellung der Optik vor dem Spektrometer (siehe Kapitel 3.3) auf die zu vermessende Probe. Eine effektive wellenlängenabhängige Effizienz der Messanordnung unter Berücksichtigung der verschiedenen Bauteile ist in Abbildung 4.4 dargestellt. Die Eingangsoptik vor dem $0.3 \mathrm{~m}$-Spektrometer besitzt eine in ihrer Beschaffenheit nicht vermeidbare chromatische Aberration, welche für unterschiedliche Wellenlängen Fokussierungen in verschiedenen Tiefen erzeugt. Dies kann dabei nicht ohne weiteres in die Berücksichtigung mit einfließen und müsste für jede Messung von neuem bestimmt werden. Beispielsweise führen unterschiedliche Probenhöhen oder auch durch temperaturabhängige Versuchsreihen hervorgerufene Änderungen der Länge des Kühlfingers, an welchen die Proben befestigt sind (siehe Kapitel 3.2), zu einer Verschiebung der Probenebene und einer damit einhergehenden lateralen als auch bedingt durch den schrägen Einfall des Lasers transversalen Änderung der Bildebenen auf dem Eingangsspalt des Spektrometers.

Nach einer vorherigen Subtraktion des Hintergrundsignals von den eigentlichen Messdaten (siehe Kapitel 4.1) lässt sich mit Hilfe der Auswertesoftware ein zweidimensionales Sensitivitätsprofil aus der z. B. in Abbildung 3.28 dargestellten Referenzmessung erstellen, welches zum direkten Ausgleich der auf der Zeitachse hervorgerufenen Intensitätsunterschiede genutzt werden kann. Alternativ ist dies auch für eindimensionale bereits zusammengefasste Bereiche möglich. Hier empfiehlt sich bestenfalls eine vorherige kurzzeitige Messung des zu beobachtenden Signals ohne eine Synchronisation der Streak-Einheit mit der eigentlichen Anregung. Dadurch entsteht eine zeitunabhängige Messung, welche als Referenz dienen kann. Aufgrund einer typischerweise langsamen Variation über den kompletten Messbereich ist beispielsweise über eine Anpassung eines Polynoms eine Beschreibung jener Variationen möglich (siehe Abbildung 4.2(a)). Aus solch einem Fit kann mittels der Messsoftware wieder ein Sensitivitätsprofil erstellt und auf die eigentlichen Messdaten angewandt werden. In Abbildung 4.3 (a) wurde das vorher extrahierte Polynom mit der selben Messdatei verrechnet, was bis auf das statistische Rauschen in einem über den kompletten Bereich konstanten Signalverlauf resultiert. Auf die eigentliche zeitabhängige Messung übertragen (siehe Abbildung 4.3(b)) führt dies dann nahezu zum kompletten Ausgleich der vorher sehr ausgeprägt sichtbaren Inhomogenitäten (siehe Abbildung 4.2(b)), hervorgerufen durch die unterschiedlichen Positionen auf dem CCD-Chip der aufzeichnenden Kamera.

Eine spektrale Sensitivitätskorrektur ist durch eine optionale Aktivierung jener Funktion für die drei verfügbaren Gitter innerhalb der Auswertesoftware möglich. Als grundlegende Korrektur sind aktuell die in der Abbildung 4.4 dargestellten Effizienzen für unpolarisiertes Licht hinterlegt. 


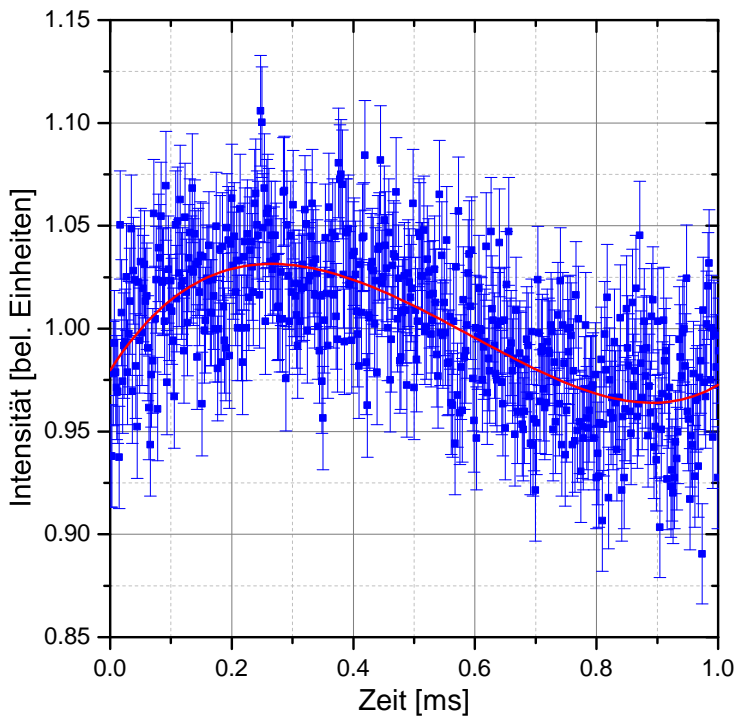

(a)

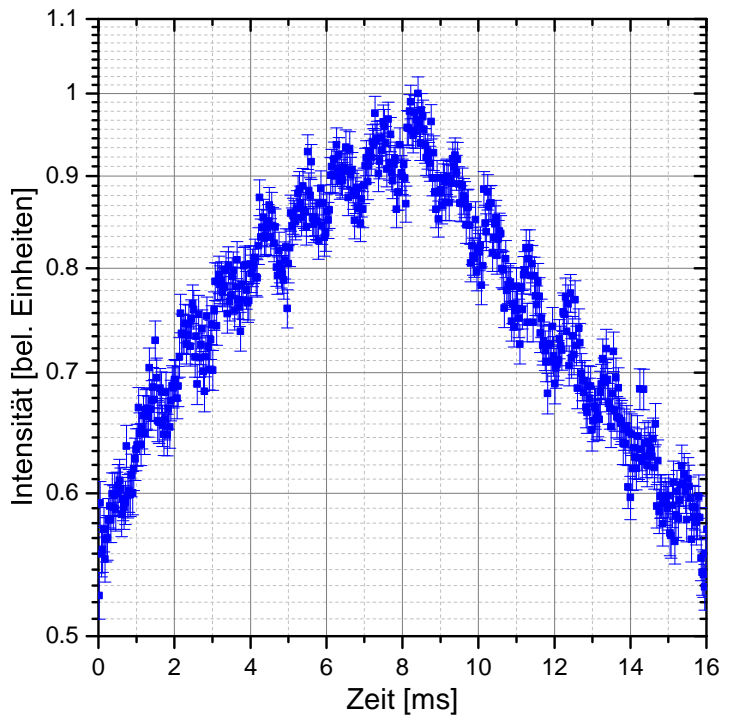

(b)

Abbildung 4.2: (a) Unkorrigierte Intensitätsverteilung eines an sich statischen Defektlumineszenz-Signals in AlN aufgenommen über ein Zeitfenster von $1 \mathrm{~ms}$ im linken Bereich der CCD-Kamera. Die rote Linie entspricht einer Anpassung über ein Polynom 5. Grades. (b) Das gleiche Signal jedoch zeitaufgelöst aufgenommen über eine Messung mit Hilfe des Trigger-Generators (siehe Kapitel 3.3.5). Die Intensität steigt durch stetiges Pumpen bis zu einer Zeit von etwa $8.2 \mathrm{~ms}$ an und sollte danach kontinuierlich abfallen.

\subsection{Zeitkorrektur}

Neben Hintergrundsignalen oder Sensitivitätsinhomogenitäten führt auch eine zeitliche Verschiebung des Trigger-Signals zu einer Verfälschung der aufgenommenen Daten. Kurzzeitiger Jitter (siehe Kapitel 3.1.5) kann aufgrund der geringen Bildwiederholfrequenz der ORCA-R2 (typischerweise $28.4 \mathrm{~Hz}$ [307]) und der geringen statistischen Rate von Ereignissen im Photoncounting-Modus im Nachhinein nicht mehr korrigiert werden. Bei einer Integration auf ein einziges Bild, was typischerweise im analogen Betriebsmodus aber auch beim Photoncounting möglich ist (siehe Kapitel 3.3.4), besteht nach einer Aufnahme nahezu keine Möglichkeit, eine zeitliche Korrektur durch eine Verschiebung der Messdaten durchzuführen. Im Gegensatz dazu können langsame Drifts oder ein Wandern innerhalb einer Messung im sequentiell aufgenommenen Photoncounting-Modus noch im Nachhinein bearbeitet werden. Abbildung 4.5 (a) zeigt die Aufnahme eines über zwei Stunden integrierten Lasersignals. Hierbei sind auch Ereignisse in Richtung größerer Zeiten zu beobachten, die aufgrund eines sich über die Zeit ändernden Triggerzeitpunktes bezüglich der Laseranregung herrühren. Noch deutlicher erscheint jener Anteil bei einer spektralen 


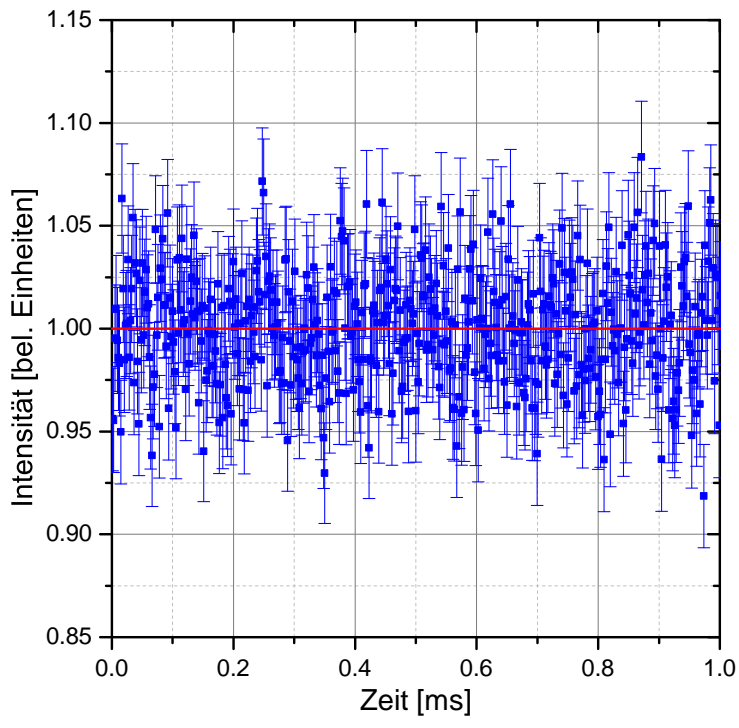

(a)

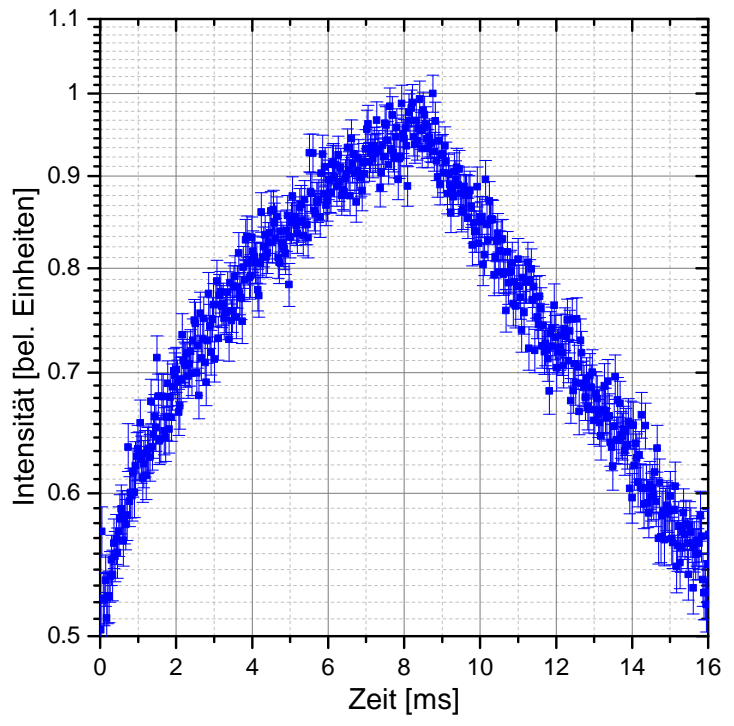

(b)

Abbildung 4.3: Gleiche Messungen wie in Abbildung 4.2, jedoch korrigiert durch eine vorher bestimmte polynomielle Anpassung. (a) Die Intensität der stationären Aufnahme ist abgesehen vom statistischen Rauschen konstant über den gesamten Messbereich verteilt. (b) Sowohl Anstieg als auch Abfall der aus 16 Zeitfenstern zusammengesetzten Messung zeigen einen kontinuierlichen Verlauf über den kompletten Aufnahmebereich.

Integration, wie in Abbildung 4.6 (a) aufgetragen. Hier könnte der Abfall sogar als vermeintliche Lebensdauer interpretiert werden. Zur Korrektur solcher zeitlicher Drifts wurden in die Auswertesoftware zwei Verfahren implementiert. Bei Messungen, welche nur einen verschwindend geringen Signalanteil an den oberen als auch unteren Rändern des Aufnahmefensters besitzen, kann eine numerische Kreuzkorrelation in Richtung der Zeitachse durchgeführt werden. Dafür wird die gesamte Aufnahme in Segmente einer bestimmten Länge (hier beispielsweise jeweils 500 Bilder) zerlegt und eine dieser Sequenzen als Referenz festgelegt. Alle weiteren Segmente werden mit jener Referenz kreuzkorreliert und der resultierende Maximalwert als Verschiebungsstrecke bestimmt. Abbildung 4.7(a) stellt die daraus berechnete Korrektur für die gesamte Aufnahmedauer dar. In dieser Messung gibt es innerhalb der ersten $30 \mathrm{~min}$ einen Drift um etwa 50 px, was einem zeitlichem Äquivalent von 130 ps entspricht. Jener resultiert aus einer Verschiebung des Trigger-Signals aufgrund sich ändernder Laserleistung und einer Erwärmung anderer Komponenten während des Betriebs. Nach dieser Anfangszeit verlangsamt sich der Drift auf etwa $2 \mathrm{px} / \mathrm{h}$. Insgesamt erscheinen die Maxima aus der numerischen Kreuzkorrelation relativ verrauscht, was aus der niedrigen Statistik innerhalb der einzelnen Zeitabschnitte resultiert. 


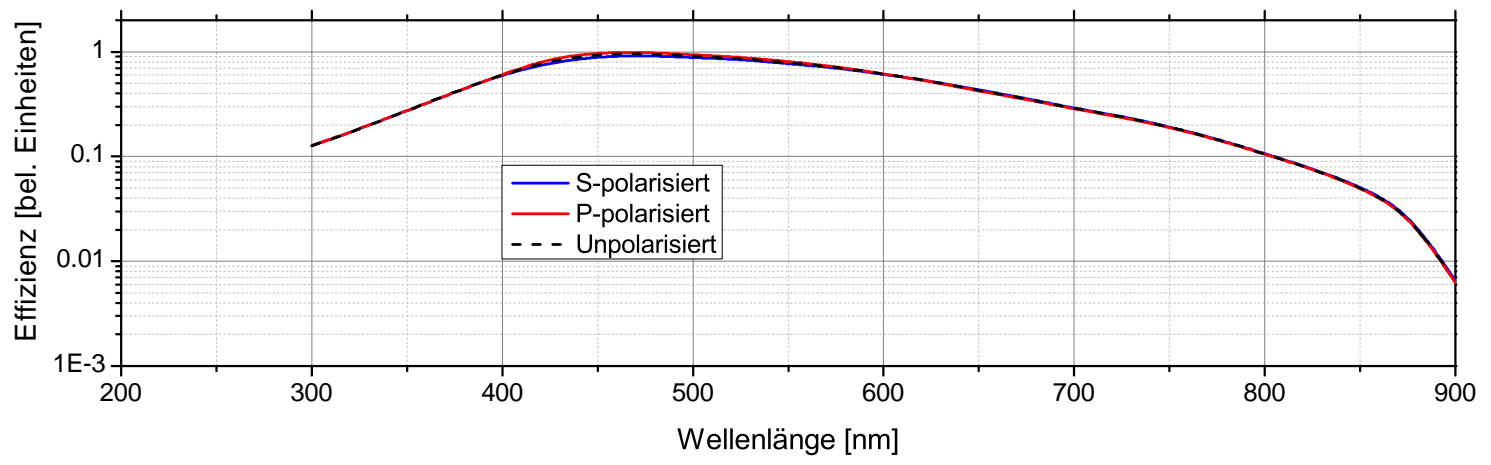

(a) Verwendetes Gitter: $50 \mathrm{~g} / \mathrm{mm}$, Effizienz ist normiert auf $3.037 \%$

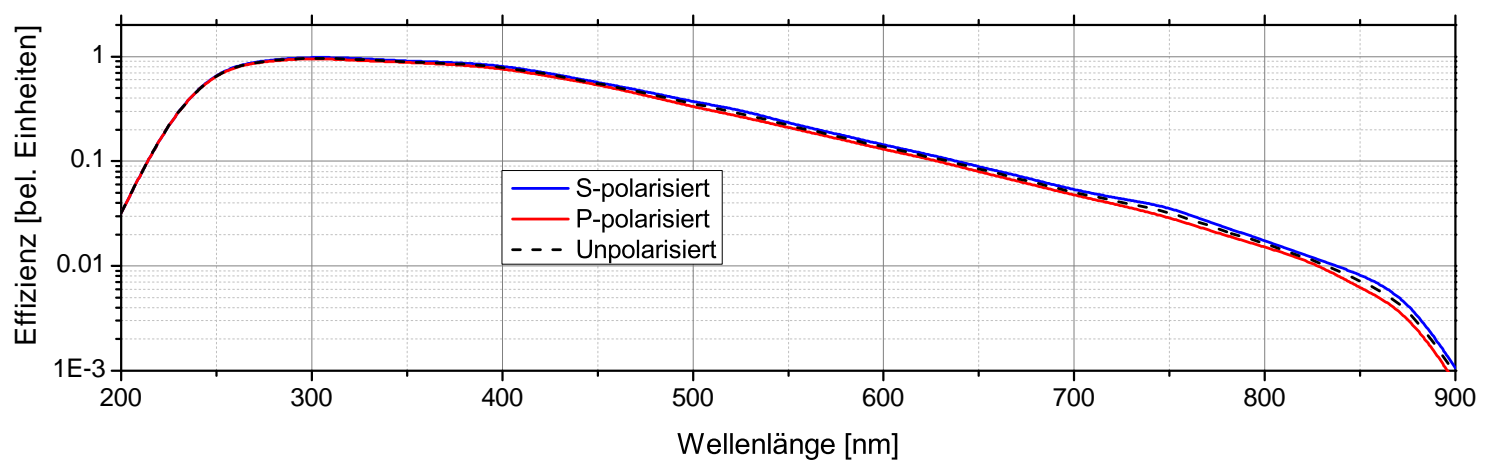

(b) Verwendetes Gitter: $300 \mathrm{~g} / \mathrm{mm}$, Effizienz ist normiert auf $3.902 \%$

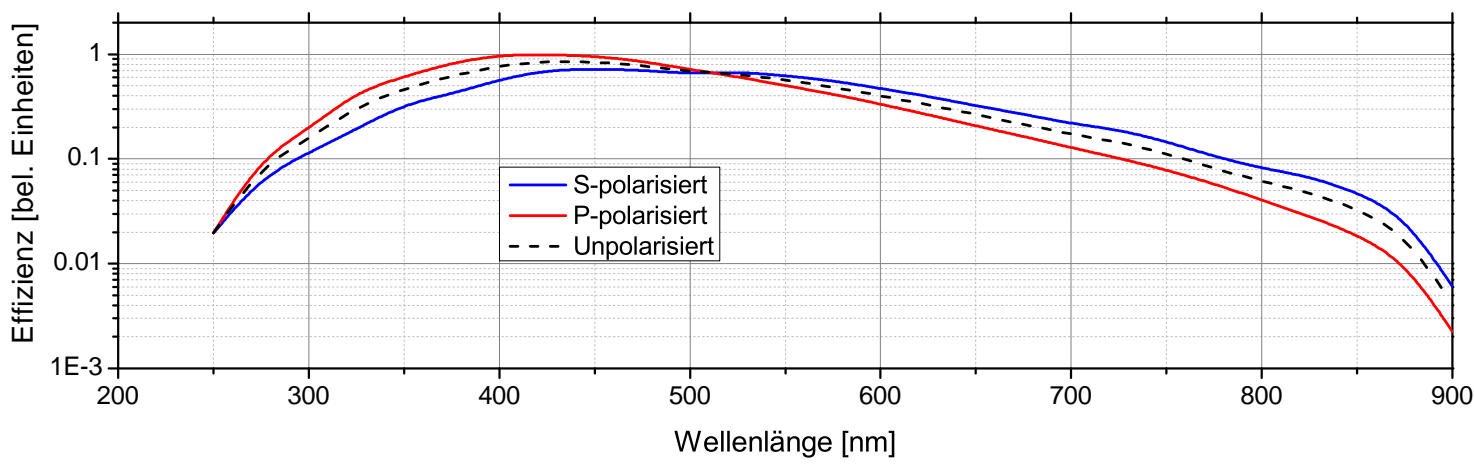

(c) Verwendetes Gitter: $900 \mathrm{~g} / \mathrm{mm}$, Effizienz ist normiert auf $4.786 \%$

Abbildung 4.4: Maximale erreichbare Effizienz des kompletten Streak-Systems unter Berücksichtigung der einzelnen Gitter ((a)-(c)), der im Spektrometer installierten Spiegel (Daten aus [297]), der Transmission der Eingangsoptik vor der Streak-Kamera und der Quanteneffizienz der Photokathode innerhalb der StreakRöhre. 
Eine Korrektur der Messdaten erfolgt anhand jener Verschiebungsdaten für jedes einzelne Bild separat unter der Nutzung einer linearen Interpolation zwischen den berechneten kreuzkorrelierten Sequenzen. Die so zeitlich verschobenen Messdaten sind in den Abbildungen 4.5(b) beziehungsweise 4.6(b) dargestellt. Insbesondere in Abbildung 4.6 (b) zeigt sich eine starke Reduktion des vermeintlichen zeitlichen Abfalls hin zu einer fast vollständig durch eine Normalverteilung beschreibbare Verteilung, welche auch für ein derart kurzlebiges Lasersignal zu erwarten ist.

Als Alternative zur Kreuzkorrelation wurde zudem noch eine Korrektur mittels funktionaler Anpassung implementiert, da ersteres Verfahren bei Messungen, welche einen signifikanten Signalanteil am zeitlichen Rand des Aufnahmefensters besitzen, im berechneten Maximalwert nicht unbedingt zu den optimalen Verschiebungsstrecken führt. Hierfür wird analog zur Kreuzkorrelation die gesamte Aufnahme in gleich lange Sequenzen aufgeteilt, eine Sequenz als Referenz erwählt und eine Anpassung einer zumindest in Teilen dem Signalverlauf entsprechenden Funktion durchgeführt. Dabei wird für jede Teilsequenz eine spektrale Integration in einem vorher festzulegenden Bereich durchgeführt. Für alle weiteren Segmente wird diese in der Abweichung minimierte Funktion (nach $\chi^{2}$-Test) jeweilig erneut angepasst, jedoch nur noch in ihrer Amplitude und dem Referenzzeitpunkt optimiert. Die daraus resultierenden Zeitnullpunkte dienen als Verschiebungsparameter und werden analog zur Kreuzkorrelation auf die Messdaten angewandt. Zum Vergleich wurde die zuvor schon präsentierte Messung des Lasersignals mit einer Normalverteilung für jedes Segment angepasst und die daraus berechneten Verschiebungsparameter in Abbildung 4.7(b) dargestellt. Der Verlauf ist der aus der Kreuzkorrelation ermittelten Verschiebung sehr ähnlich, zeigt jedoch ein vermindertes statistisches Rauschen aufgrund höherer Zählraten durch die lediglich eindimensionalen Anpassungen.

Die zeitlichen Verschiebungen des Trigger-Signals über lange Messzeiten bei Aufnahmen unter der Nutzung der Trigger-Diode und des Pulspickers besitzen typischerweise Werte in der Größenordung um 100 ps und sind somit nur für Zeitfenster mit einer Länge $\leq 10$ ns von Interesse. Bei Messungen mit dem Chopperrad liegen die Verschiebungen jedoch je nach Laufgeschwindigkeit im Bereich von $10-100 \mu$ s und sind somit auch bei langen Zeitfenstern von bis zu $1 \mathrm{~ms}$ zu registrieren.

\subsection{Krümmungskorrektur}

Bei breitbandigen Lumineszenzen oder einem gezielten zeitlichen Vergleich von mehreren innerhalb einer Messung aufgenommen Signalen, welche sich lateral weit über das komplette Aufnahmefenster erstrecken, empfiehlt sich eine zusätzliche Korrektur bezüglich unterschiedlicher Zeitnullpunkte. Wie in Abbildung 4.8(a) dargestellt besitzt die Streak-Kamera bauartbedingt eine leichte Krümmung der Zeitachse entlang der fortlaufenden lateralen Position innerhalb des Aufnahmefensters. In der Auswertesoftware wurde zur Korrektur jener Abweichung die Möglichkeit einer 


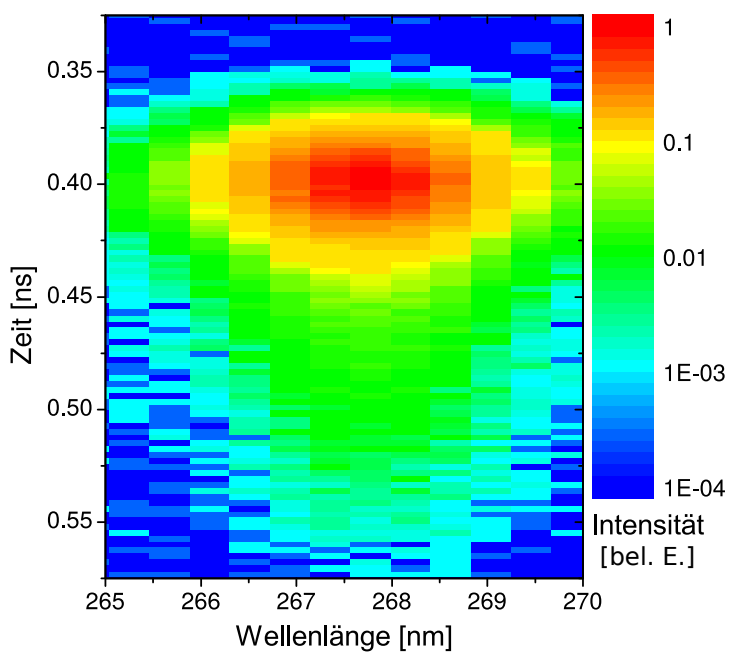

(a)

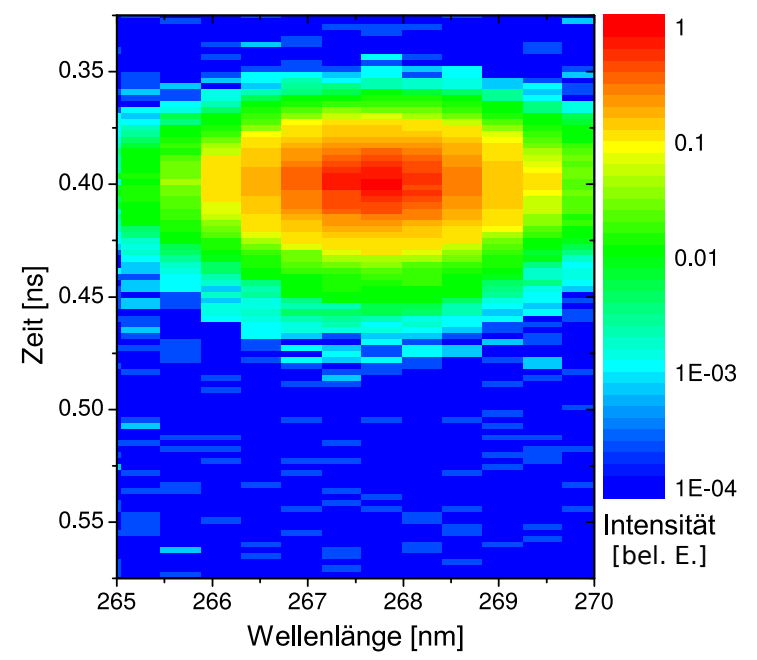

(b)

Abbildung 4.5: (a) Aufnahme einer dritten Harmonischen integriert über einen Zeitraum von zwei Stunden. Es ist eine Ausschmierung des Signals hin zu größeren Zeiten zu beobachten. (b) Das gleiche Signal nur mit Hilfe einer Kreuzkorrelation kontinuierlich in seiner Zeit nachjustiert. Die Verschiebung der einzelnen Ereignisse erfolgte dabei nach der Auftragung in Abbildung 4.7.

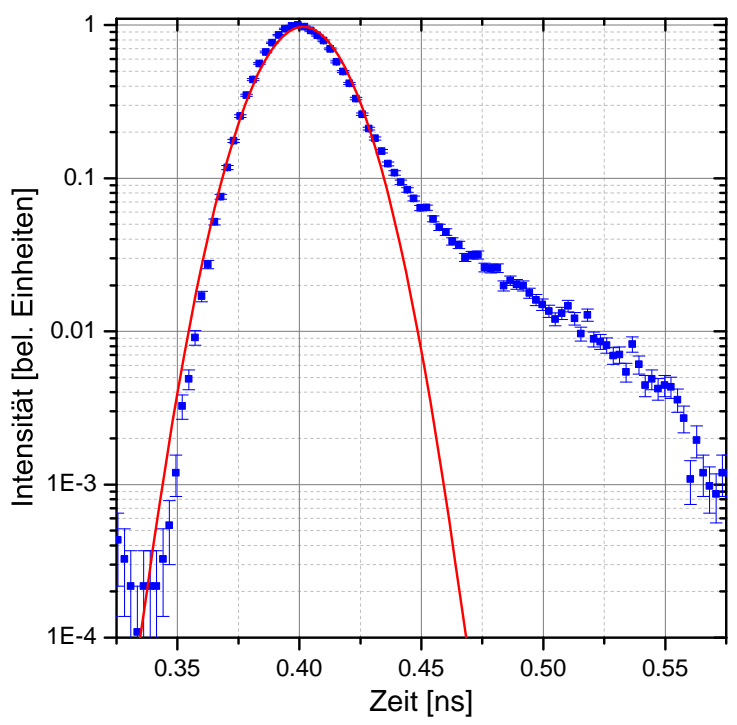

(a)

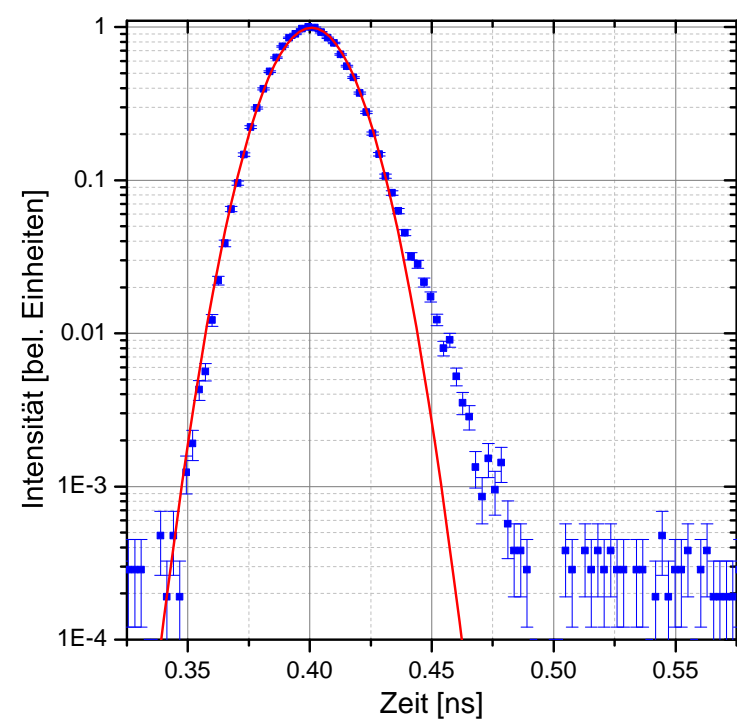

(b)

Abbildung 4.6: Gleiche Signale wie in Abbildung 4.5 nur zusätzlich über einen Wellenlängenbereich von 265-270 nm integriert. Bei den roten Kurven handelt es sich um an die Messdaten angepasste Normalverteilungen. Dabei beträgt der Anteil der nicht von der Funktion abgedeckten Fläche bei (a) $7.1 \%$ und bei (b) $1.0 \%$. 


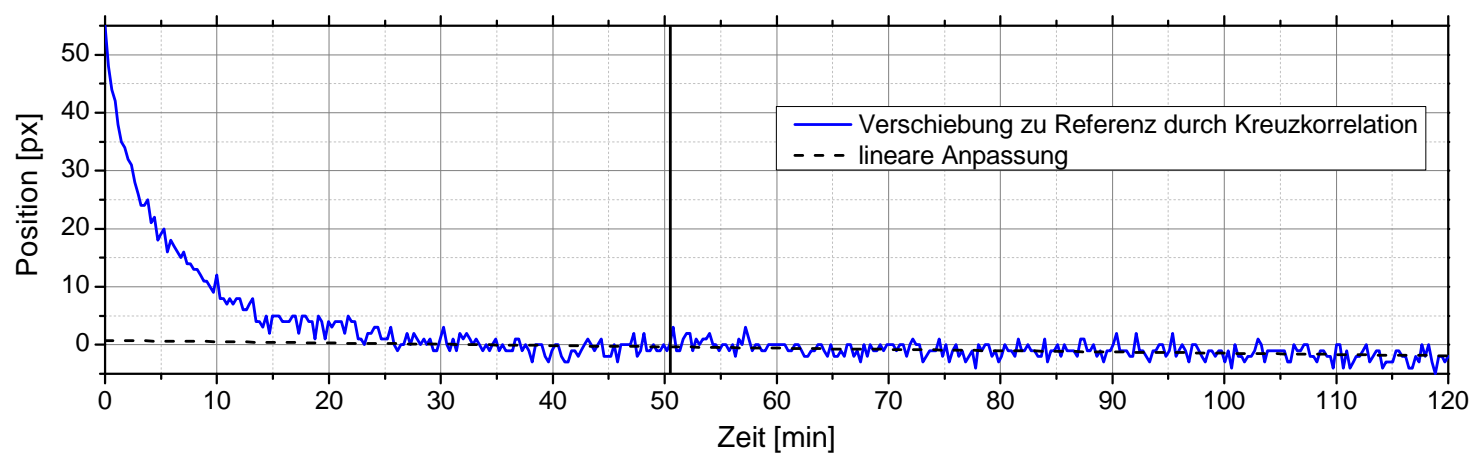

(a)

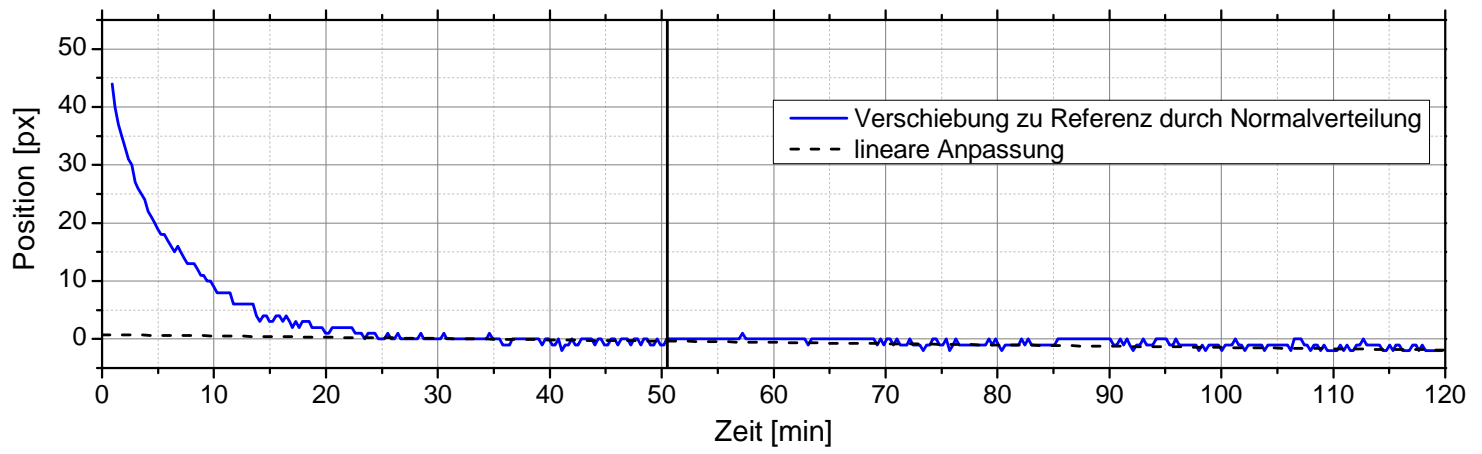

(b)

Abbildung 4.7: Zeitlicher Verlauf der Korrektur aus der berechneten Kreuzkorrelation (a) als auch einer skalierten Verschiebung einer Normalverteilung (b). Als Referenz dient eine Aufnahme von 500 Bildern bei einer Zeit von 51 min (schwarze senkrechte Linien). Die ersten 30 min ist jeweils ein starker Drift über fast 50 Pixel zu beobachten. Danach verlangsamt sich jener und führt zu einem allmählichen Wandern mit einer Tendenz weiter hin zu früheren Zeiten. Die linearen Anpassungen erfolgten ab einer Zeit von 30 min. Beide Varianten liefern ein äquivalentes zeitliches Verhalten, wobei bei der Kreuzkorrelation durch eine Anwendung auf das zweidimensionale Feld ein höherer statistischer Rauschwert auftritt. Dem hingegen konvergiert die automatisierte Anpassung der Normalverteilung für die ersten drei Bilder nicht im korrekten Bereich, wodurch diese Werte fehlen. 
Interpolation mittels eines kubischen Polynoms implementiert. Dafür wird innerhalb eines oberen Aufnahmebereichs für jede Spalte der Schwerpunkt gebildet und das Polynom entlang der einzelnen Schwerpunkte angepasst. Die daraus resultierenden Parameter können zur Verschiebung der einzelnen Spalten und einer anschließenden neuen anteiligen Zuordnung wieder zu einer Gesamtmessung zusammengeführt werden. Das so korrigiertes Signal ist in Abbildung 4.8(b) gezeigt.

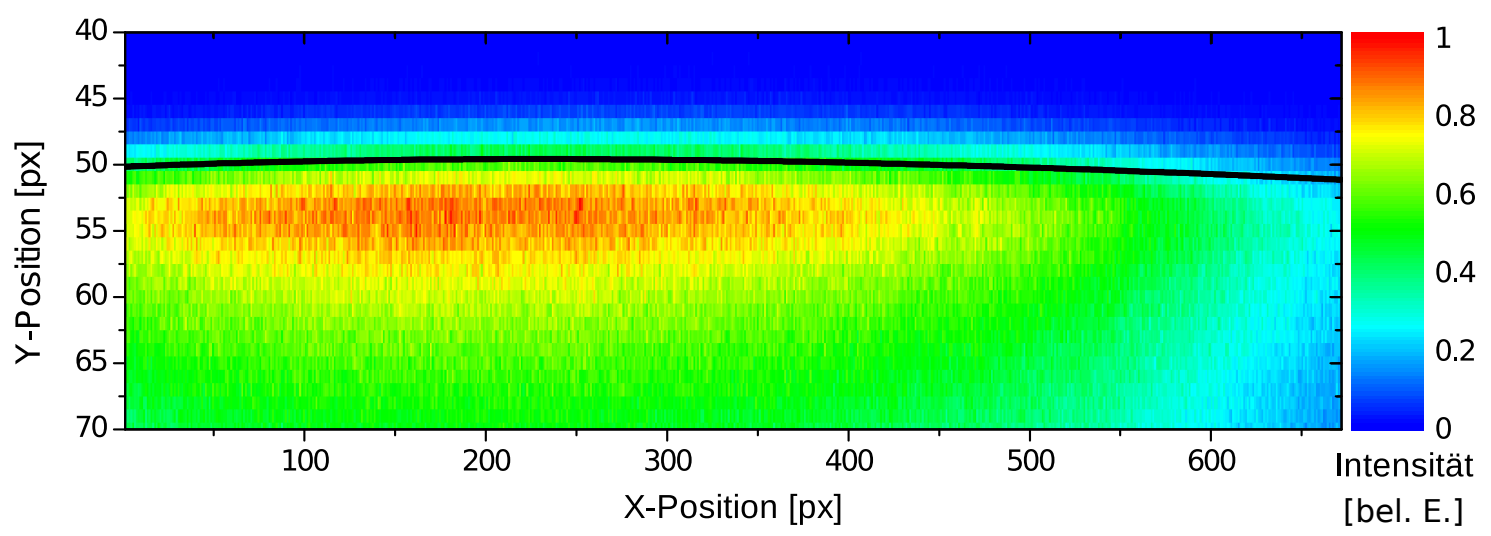

(a)

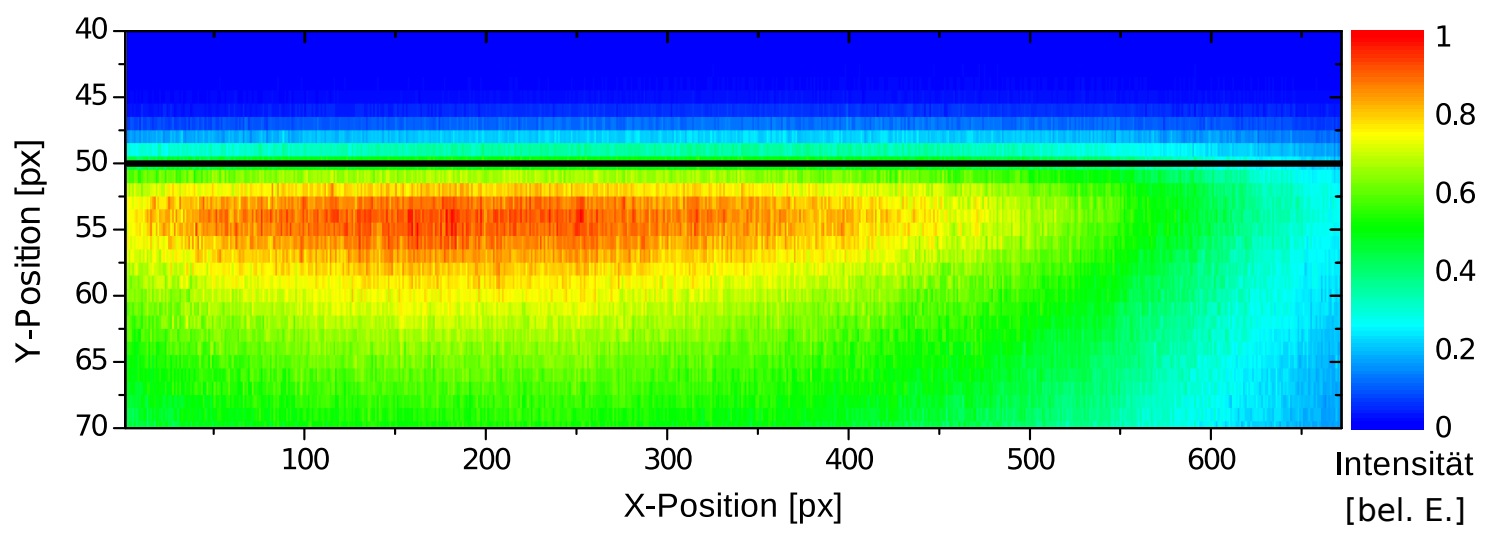

(b)

Abbildung 4.8: (a) Ausschnitt einer Aufnahme einer breitbandigen Lumineszenz von Fluorescein in einem 20 ns Zeitfenster. Die kubisch angepasste Krümmung (schwarze Kurve) besitzt über den gesamten Bereich eine maximale Verschiebung von $1.55 \mathrm{px}$, was etwa $0.3 \%$ des gesamten Zeitfensters entspricht. (b) Durch die angepasste Kurve korrigierte Aufnahme. Die einzelnen Spalten wurden um die entsprechende Position Richtung der y-Achse verschoben und anteilig neu auf die Pixelmatrix aufsummiert. 


\title{
4.5 Verkippung
}

Da die CCD-Kamera generell drehbar gelagert separat an der Streak-Einheit befestigt ist, kann zwischen der Sweep-Richtung und der senkrechten Pixelanordnung auf dem CCD-Chip eine kleine Winkeldifferenz auftreten. Jene Verkippung führt im aktuellen Montagezustand zu einer Verschiebung des Signals vom obersten Bereich bis hin zur unteren Kante des CCD-Chips um 2.45(17) px. Gemessen an der Gesamtbreite des Aufnahmefensters entspricht dies zwar nur einem Anteil von $0.36 \%$ oder einem Winkel von $0.27^{\circ}$, jedoch ist bei schmalbandigen sich über den gesamten Messbereich erstreckenden Signalen eine solche Verschiebung des Schwerpunktes gut auszumachen (siehe Abbildung 4.9(a)). Bei einer zeitlichen Integration der Lumineszenzen über den kompletten Messbereich hinweg führt dies zu einer leichten Verschlechterung der spektralen Aufösung. Da es sich um eine lineare Abweichung handelt, ist eine stückweise Neupositionierung des Messsignals analog zur Korrektur der Krümmung (siehe Kapitel 4.4) möglich. In Abbildung 4.9(b) ist das gleiche Signal nach einer kontinuierlichen Verschiebung dargestellt. Eine vollständige Wiederherstellung der spektralen Auflösung ist über dieses Verfahren dabei nicht möglich, da ein kontinuierliches Versetzen um lediglich Anteile eines Pixels zu einer Aufweitung des gesamten Signals um maximal 1 px führen kann.

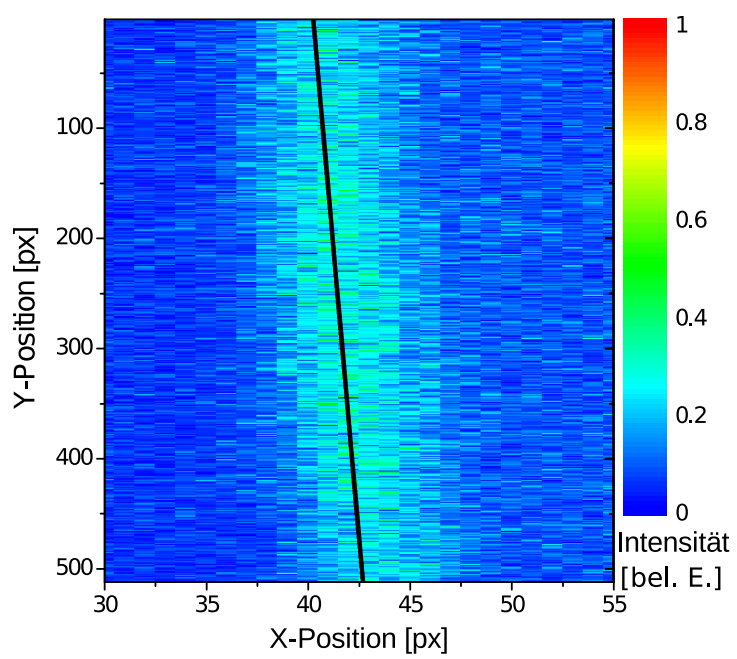

(a)

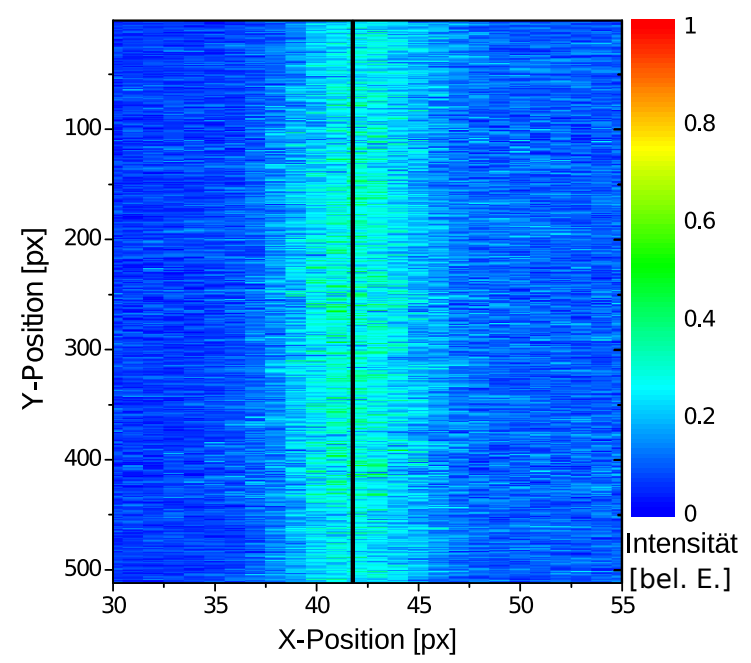

(b)

\begin{abstract}
Abbildung 4.9: (a) Ein sich über den gesamten Messbereich erstreckendes statisches Signal einer $E u^{3+}$-Lumineszenz. Über die vertikale Position auf dem CCD-Chip hinweg verschiebt sich das Zentrum um 2.45(17) px. (b) Linear korrigierte Verkippung der schmalbandigen Lumineszenz. Das Messsignal zeigt nun nahezu keine Verschiebung innerhalb des kompletten Fensterbereiches mehr.
\end{abstract}




\subsection{Erweiterte Zeitfenster}

Die Neuimplementierung einer Messung von Zeitfenstern länger als 1 ms mit Hilfe eines in dieser Arbeit dafür entwickelten Trigger-Generators (siehe Kapitel 3.3.5) bedarf bei der Datenanalyse einer speziellen Auswertemethodik, da die Rohdaten sich von einer gewöhnlichen sequentiellen Photoncounting-Messung nicht unterscheiden. Aufgrund des Aufnahmemusters können die einzelnen Messbereiche jedoch aus den Rohdaten im Nachhinein extrahiert werden. In Abbildung 4.10 ist der Ausschnitt eines Teils einer Sequenz dargestellt. Zwischen den verschiedenen Aufnahmebereichen (hier jeweils $10 \mathrm{~s}$ ) pausiert die Messung für eine kurze Zeit (hier $300 \mathrm{~ms}$ ). Innerhalb dieser Zeiten werden bei nicht zu niedrig eingestelltem Schwellwert keine Ereignisse im Streak-System erfasst. Über die Auswertesoftware können jene Stellen registriert und anschließend eine Aufteilung und Integration der einzelnen Segmente über die gesamte Messung erfolgen. Hierbei spielt die Wahl der Position des ersten Elements eine entscheidende Rolle, da jenes auch in den resultierenden Daten an erster Position steht und gegebenenfalls keine kontinuierliche Messkurve entstehen würde, wenn beispielsweise ein Segment in der Mitte eines Zerfalls an diese Stelle tritt. Die Anzahl der genutzten Bins zur Integration entspricht dabei der einer gewöhnlichen Photoncounting-Messung, sodass die daraus resultierende Matrix der Messdaten die gleiche Größe besitzt. Da bei solchen Aufnahmen stets der gesamte zeitliche Bereich des in der Messung real existierenden Messfensters genutzt wird, ist eine Korrektur der Sensitivitätsverteilung unablässig (vergleiche hierzu Abbildung 4.2(b)). Die entsprechende Bestimmung der Sensitivität für die jeweiligen Bereiche wird bei vorhandener Korrekturdatei in der Auswertesoftware automatisch durchgeführt.

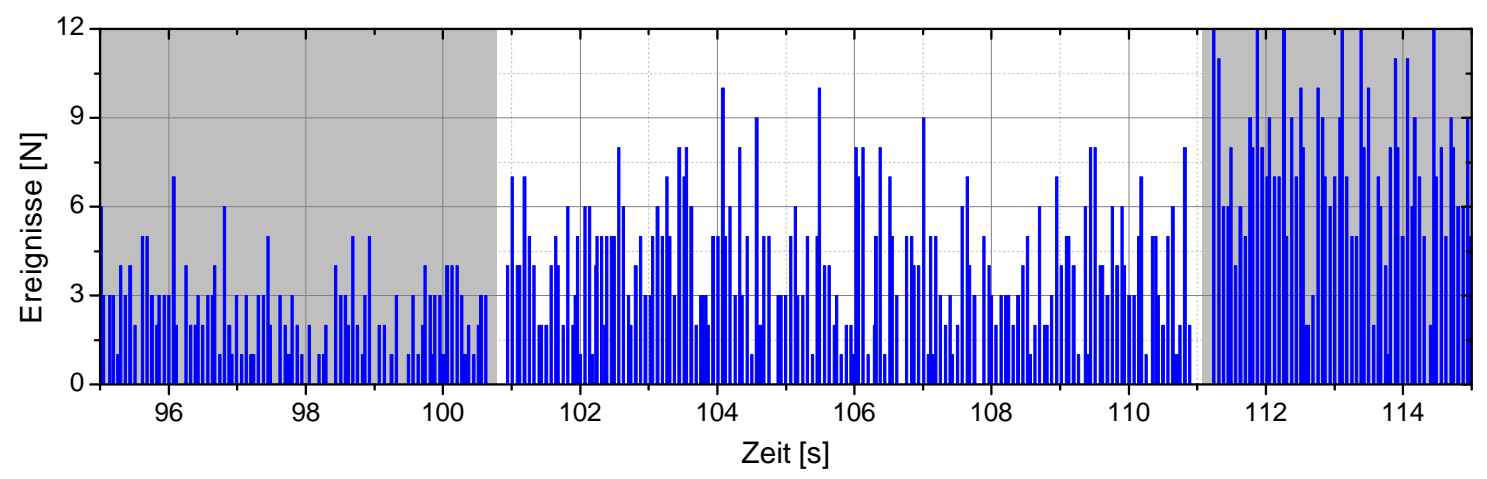

Abbildung 4.10: Ausschnitt der Zählrate pro Bild einer Messung mit dem TriggerGenerator in Verbindung mit dem Chopperrad (siehe Kapitel 3.3.5). Nach jeweilig einem Messintervall von etwa $10 \mathrm{~s}$ (unterschiedlich hinterlegte Bereiche) erfolgt eine Messpause von $300 \mathrm{~ms}$ und eine anschließende Messung des nächsten Intervalls, was zu verschiedenen durchschnittlichen Zählraten innerhalb jener führt. 


\subsection{Ratengleichungen}

Die im Rahmen dieser Arbeit zeitlich vermessenen Lumineszenzanregungen folgen zumeist einem Verlauf, welcher mit Übergangsraten nach exponentieller Form gut abgebildet werden kann. Die dabei primär betrachteten Fälle beziehen sich hauptsächlich auf Rekombinationen, die direkt aus dem angeregten Energielevel zurück in den Grundzustand erfolgen, wobei eine eventuelle Transferzeit in den oberen Zustand unberücksichtigt bleibt (siehe Abbildung 4.11(a)). Sekundär werden auch Modelle mit einer verglichen zur Lebensdauer der lumineszierenden Rekombination signifikanten vorherigen Transferzeit angewandt (siehe Abbildung 4.11(b)). In beiden Modellen wird für jeden Übergang zu einem niedrigerem Niveau von einer konstanten Rate $k$ ausgegangen. Im Allgemeinen erhalten die Ratengleichungen im Fall nur eines Anregungsniveaus für jenes und den Grundzustand folgende Form:

$$
\begin{aligned}
& \frac{\mathrm{d} A(t)}{\mathrm{d} t}=p(t)-k_{A} \cdot A(t) \\
& \frac{\mathrm{d} G(t)}{\mathrm{d} t}=k_{A} \cdot A(t)-p(t),
\end{aligned}
$$

wobei hier $p(t)$ für die zeitlich abhängige Pumpleistung in das obere Niveau und $k_{A}$ für dessen Transferrate in das untere stehen. Inklusive eines Zwischenlevels besitzen die Ratengleichungen folgende Gestalt:

$$
\begin{aligned}
& \frac{\mathrm{d} A(t)}{\mathrm{d} t}=p(t)-k_{A} \cdot A(t) \\
& \frac{\mathrm{d} B(t)}{\mathrm{d} t}=k_{A} \cdot A(t)-k_{B} \cdot B(t) \\
& \frac{\mathrm{d} G(t)}{\mathrm{d} t}=k_{B} \cdot B(t)-p(t) .
\end{aligned}
$$

Die Funktionen $A, B$ und $G$ beschreiben die jeweiligen Besetzungszahlen der einzelnen Niveaus, wobei für die oberen beiden stets $A(-\infty)=0$ beziehungsweise $B(-\infty)=0$ gilt. Für den Grundzustand wird von einer gegenüber der Anregungsleistung großen Besetzungszahl ausgegangen, sodass eine mögliche Entleerung jenes Zustandes nicht berücksichtigt wird. Im Betrieb mit Trigger-Diode und Pulspicker kann der Anregungspuls aufgrund seiner im Verhältnis zu den Längen der Aufnahmefenster verschwindend geringen Dauer von $\approx 130$ fs (siehe Kapitel 3.1.2) im einfachsten Fall als infinitesimal kurzer Puls, welcher zu einer Besetzung des oberen angeregten Zustandes zum Startzeitpunkt $t_{0}$ von $A\left(t_{0}\right)=A_{0} / k_{A}$ führt, charakterisiert werden. Unter Berücksichtigung der zeitlichen Auflösung ist auch eine Beschreibung über eine Normalverteilung der Form:

$$
p(t)=\frac{p_{0}}{k_{A} \sqrt{2 \pi \sigma^{2}}} \exp \left(-\frac{\left(t-t_{0}\right)^{2}}{2 \sigma^{2}}\right)
$$




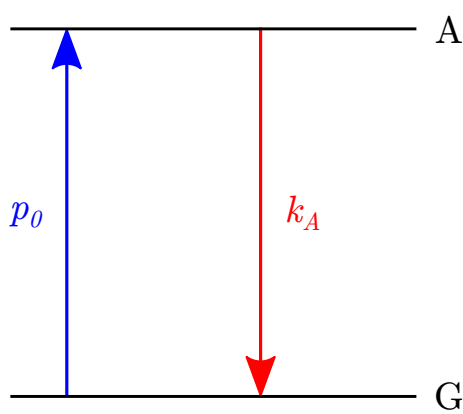

(a) Direkter Zerfall

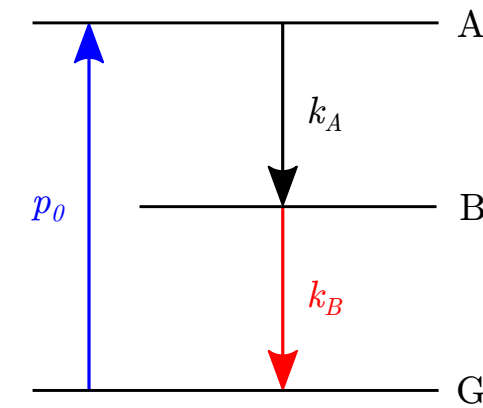

(b) Mit Zwischenniveau

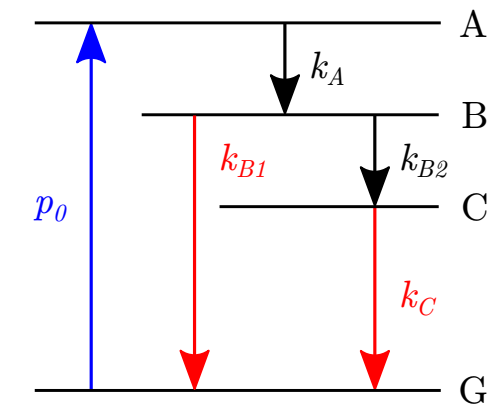

(c) Komplexe Kaskade

Abbildung 4.11: Verschiedene Zerfallsschemata mit einer maximal anregenden Generierungsrate $p_{0}$. Die Übergangsrate von den jeweilig höher gelegenen Niveaus ("A", "B", "C") in energetisch niedrigere Anregungszustände oder den Grundzustand "G" wird mit $k_{A}, k_{B}$ und $k_{C}$ angegeben.

angebracht, wobei hier $\sigma$ für die Standardabweichung und $p_{0}$ für eine beliebige Referenzleistung stehen. Im Betrieb mit Chopperrad dienen hingegen, im Fall ohne einer Einbeziehung der zeitlichen Auflösung, Stufenfunktionen der Beschreibung sich öffnender beziehungsweise schließender Fenster des Chopperrads. Bei langen Zeitfenstern unter der Verwendung des Trigger-Generators (siehe Kapitel 3.3.5), da sowohl Anstieg als auch Abfall beobachtbar sind, eine Rechteckfunktion der Breite $t_{A}$. Zur Berücksichtigung der zeitlichen Auflösung werden diese Funktionen mit einer Normalverteilung nach Gleichung 4.6 gefaltet. Alle hier betrachteten Anregungsformen sind in Abbildung 4.12 schematisch dargestellt. Die aus den Differentialgleichungen 4.1-4.5 folgenden zeitabhängigen Lumineszenzen für die verschiedenen vorgestellten Anregungsfälle sind im Anhang in den Gleichungen A.1-A.12 angegeben. Für die Wahrung einer besseren Übersicht wurde bei den Funktionen ein Anregungszeitpunkt von $t_{0}=0$ gewählt. Ermittelt wurden jene Lösungen unter anderem durch die Zuhilfenahme der Software Mathematica (Wolfram Research, Inc.) [313]. Dabei konnten die Algorithmen keine analytische Lösung für Zerfälle mit Zwischenniveau in Kombination mit einer Normalverteilung gefalteten Anregung bestimmen. Während bei kurzlebigen Zerfällen mit Dauern im Bereich der zeitlichen Auflösung eine Berücksichtigung jener große Unterschiede in den ermittelten Zerfallskonstanten liefert, spielt sie bei im Verhältnis dazu langlebigen Prozessen nur eine untergeordnete Rolle. Eine grafische Übersicht der verschiedenen gefundenen Lösungen ist in Abbildung 4.13 gegeben. Für die bei einem Fit eventuell möglichen freien Variablen wurden $\operatorname{dort} k_{A}=5, k_{B}=4, \sigma=0.1$ und $t_{A}=0.75$ gewählt. Hier ist zu beobachten, dass bei einer Unberücksichtigung der zeitlichen Auflösung die Funktionen jeweils am Zeitnullpunkt signifikante Änderungen innerhalb ihrer Verläufe aufweisen (siehe Abbildung 4.13 erste und zweite Spalte), während mit Zeitauflösung diese Punkte keinen auffälligen Charakter mehr zeigen (siehe Abbildung 4.13 dritte Spalte). 


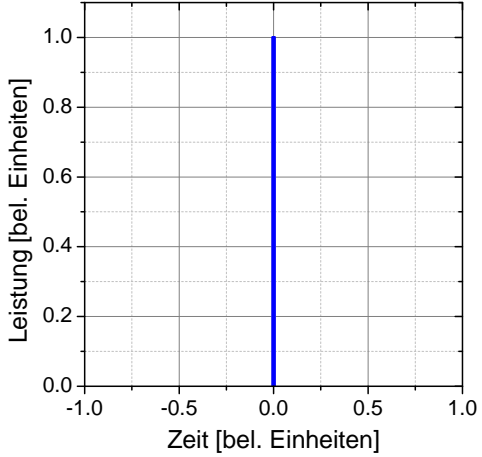

(a) Puls

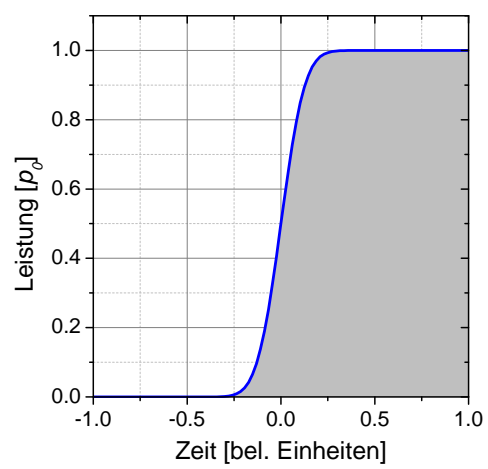

(d) Fehlerfunktion Anstieg

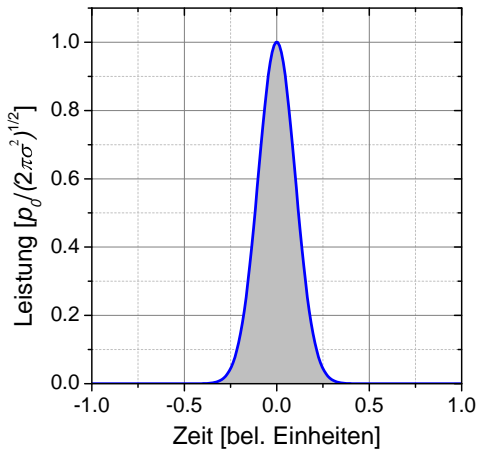

(b) Normalverteilung

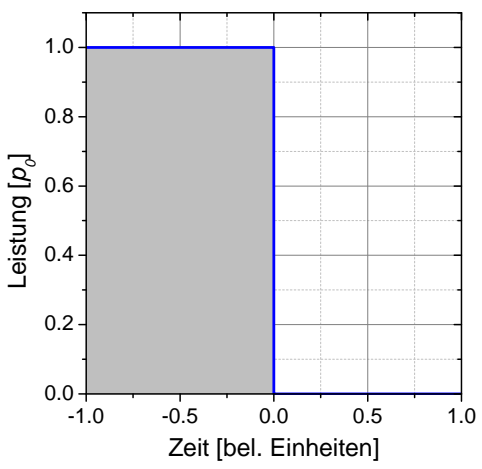

(e) Stufenfunktion Abfall

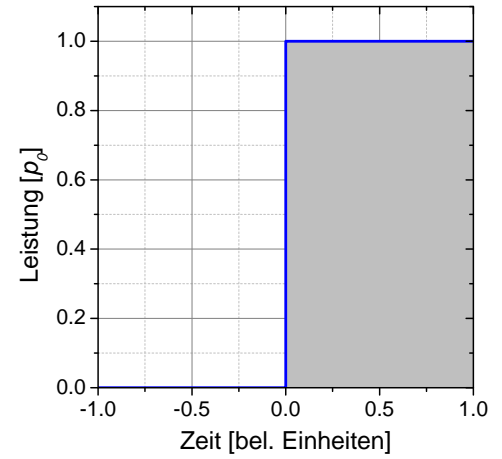

(c) Stufenfunktion Anstieg

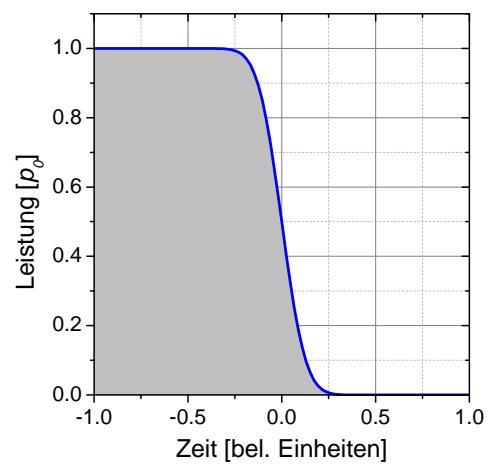

(f) Fehlerfunktion Abfall

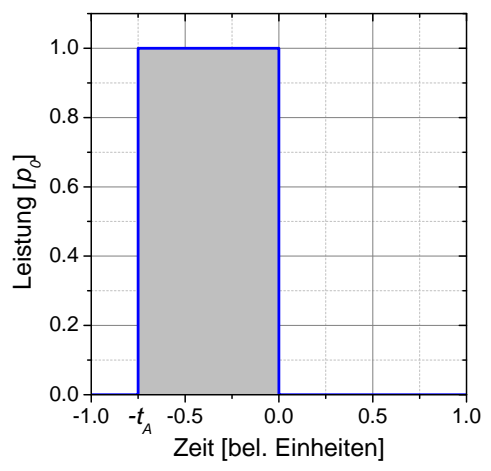

(g) Box-Anregung

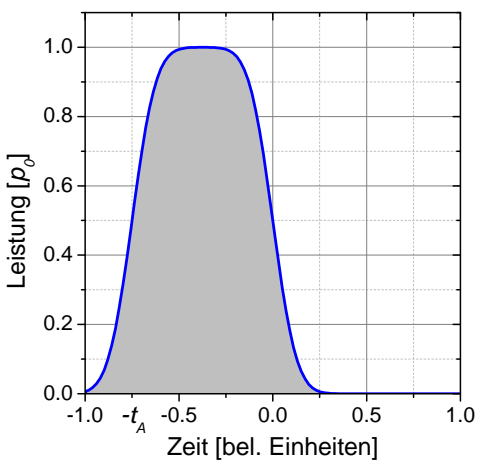

(h) Box + Fehlerfunktionen

Abbildung 4.12: Anregungsmodelle für verschiedene Szenarien. Die Modelle (a) und (b) kommen bei der Auswertung von Messdaten, bei denen mit der TriggerDiode und dem Pulspicker gearbeitet wurde zum Einsatz. Bei Aufnahmen mit Hilfe des Chopperrads in Zeitfenstern bis 1 ms finden typischerweise (c) - (f) ihre Verwendung. Für Messungen unter Nutzung des Trigger-Generators können je nach Einstellung Anstieg als auch Abfall innerhalb eines langen Zeitfensters simultan und damit direkt gekoppelt ausgewertet werden. 


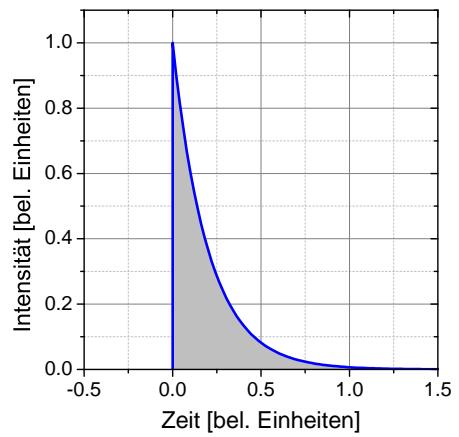

(a) Puls direkt

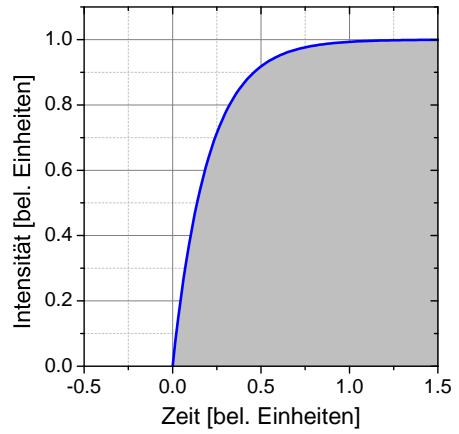

(d) Stufe direkt

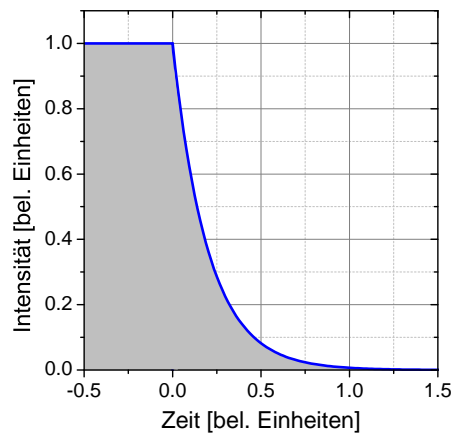

(g) Stufe direkt

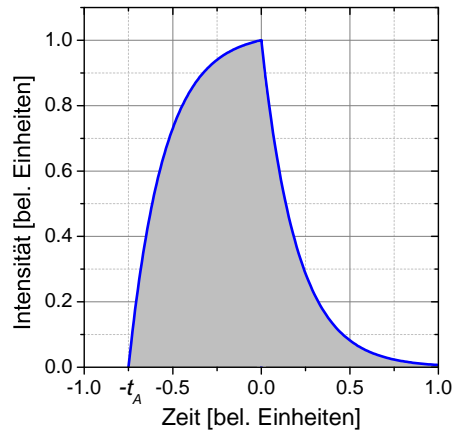

(j) Box direkt

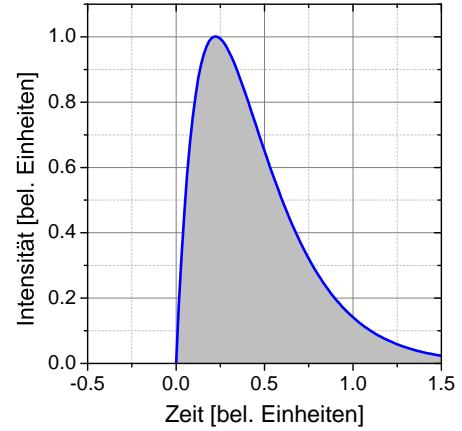

(b) Puls mit Zwischenniveau

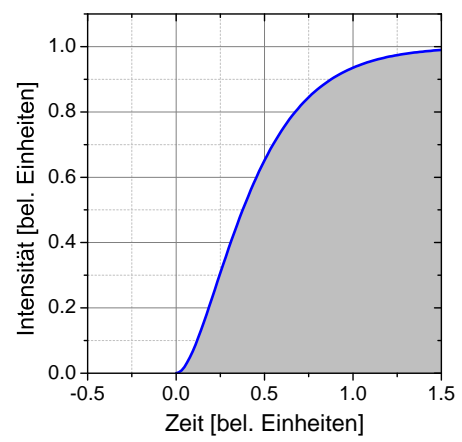

(e) Stufe mit Zwischenniveau

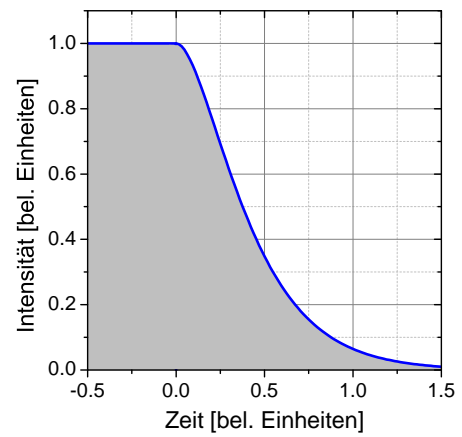

(h) Stufe mit Zwischenniveau

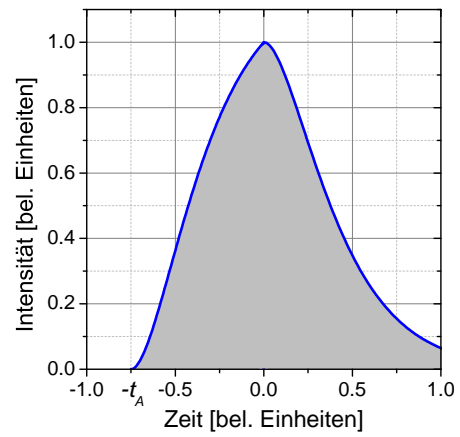

(k) Box mit Zwischenniveau

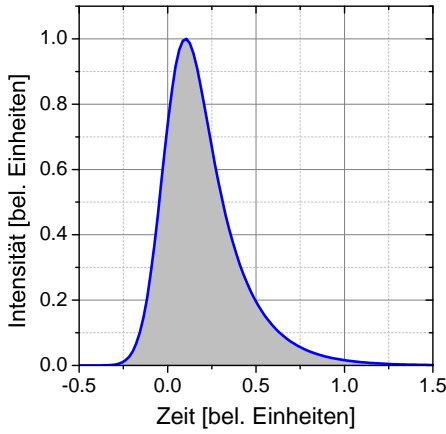

(c) Normalverteilung direkt

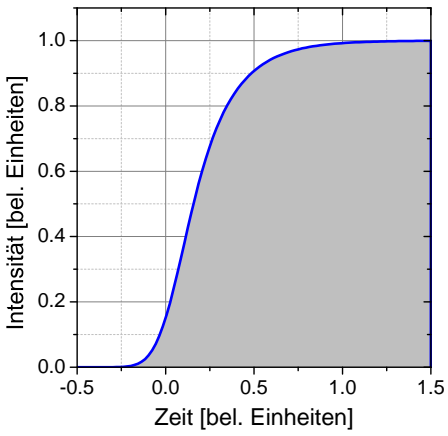

(f) Fehlerfunktion direkt

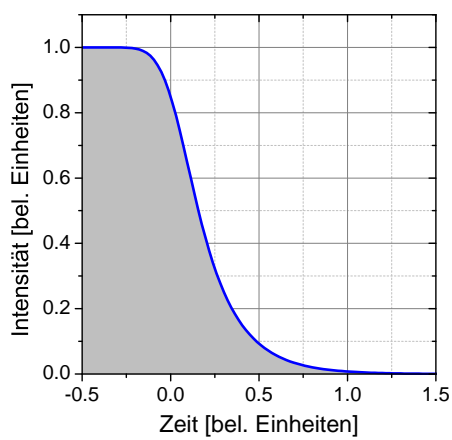

(i) Fehlerfunktion direkt

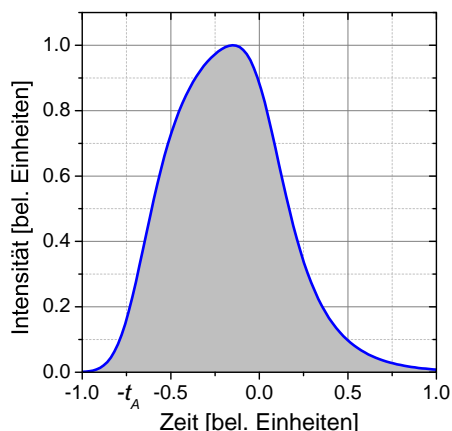

(l) Fehlerfunktion-Box direkt

Abbildung 4.13: Beispielhafte Kurvenverläufe der Gleichungen A.1-A.12 aus den Zerfallsschemata von Abbildung 4.11 und den Anregungen aus Abbildung 4.12. 
Als Beispiel einer Messung einer komplexeren Zerfallskaskade sei an dieser Stelle das Lumineszenzverhalten von $\mathrm{Ho}^{3+}$ in $\mathrm{YVO}_{4}$ Nanokristallen vorgestellt. Der Energietransfer folgt dabei in vereinfachter Form dem Zerfallsschema nach Abbildung 4.11(c) und führt zu zwei Lumineszenzbändern bei etwa $2.3 \mathrm{eV}$ und $1.9 \mathrm{eV}$. Die in Abbildung 4.14 dargestellten Intensitätsverläufe entstammen aus einer Messung mit dem 50er Gitter und konnten so simultan aufgenommen werden. Auffällig zeigt sich hier ein verspäteter Anstieg des Signals, welches dem Transfer ${ }^{5} F_{5} \rightarrow{ }^{5} I_{8}$ zugeordnet ist, da jener Übergang hauptsächlich durch das früher ansteigende ${ }^{5} F_{4}$-Niveau populiert wird. Rein augenscheinlich scheinen beide Zerfälle in etwa die gleichen Lebensdauern zu besitzen. Jedoch zeigt sich bei einer Lösung des Schemas aus 4.11(c) eine davon sehr abweichende Interpretation. Durch eine gleichzeitige Anpassung der Gleichungen in A.13 ergibt sich eine fast vier mal so schnelle Übergangsrate von dem ${ }^{5} F_{5}$-Level in das Grundniveau verglichen zum ${ }^{5} F_{4}$-Niveau. Die komplette Anpassung basiert auf lediglich 5 freien Parametern, wobei bei einer Kenntnis der genauen relativen Intensität zwischen den beiden Zerfällen auch eine Unterscheidung der Transferraten $k_{B 1}$ und $k_{B 2}$ getroffen werden könnte. Der anfängliche unsichtbare Übergang in das obere der beiden leuchtenden Niveaus beträgt 3.51(5)/ $\mu$ s was einer Transferzeit von $0.285(4) \mu$ s entspricht. Für das untere der beiden Level scheint zu Beginn des Anstiegs die Anpassung den Signalverlauf leicht zu unterschätzen, was auf einen weiteren Transfer in dieses Niveau schließen lässt. Dieser kann jedoch aufgrund der nur geringen Diskrepanz eine lediglich leicht größere Rate, als der berücksichtigte Transfer aufweisen.

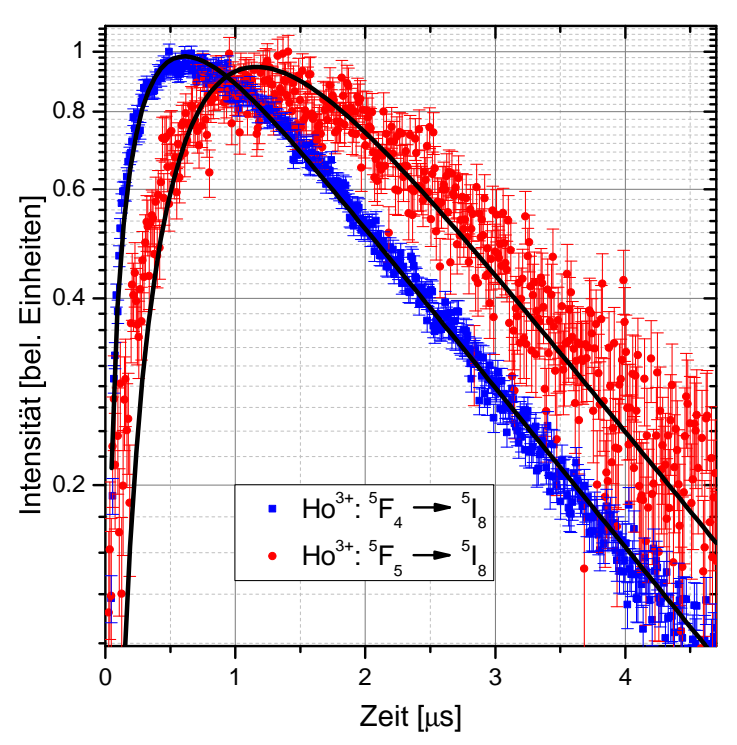

Abbildung 4.14: Anpassung von Gleichung A.13 nach dem Schema 4.11(c) mit angenommener kurzer pulsförmiger Anregung für eine $\mathrm{Ho}^{3+}{ }_{-}$Lumineszenz in $\mathrm{YVO}_{4}$ Nanokristallen. Die beiden Zerfallskurven wurden, da physikalisch miteinander gekoppelt, simultan für 5 freie $\mathrm{Pa}$ rameter (2 sichtbare Zerfallsraten, eine nicht sichtbare Transferrate und 2 Intensitäten) angepasst. Für die Kurven ergibt sich jeweils eine Rate von $k_{B 1}+k_{B 2}=$ $0.592(4) / \mu \mathrm{s}$ und $k_{C}=2.33(6) / \mu \mathrm{s}$ (rot). 



\section{Experimentelle Untersuchung von Aluminiumnitrid}

Im folgenden Kapitel wird auf das Lumineszenzverhalten von über die HPHTMethode hergestellten AlN-Einkristalle (siehe Kapitel 5.1) näher eingegangen. Zunächst werden das Herstellungsverfahren und die Eigenschaften der Proben dafür kurz vorgestellt. Darauf folgen drei Abschnitte, die sich jeweilig mit einem anderen Lumineszenztyp beschäftigen. Zunächst werden die beobachteten breitbandigen Defektlumineszenzen untersucht (Kapitel 5.2). Darauf folgt ein Abschnitt, der sich mit einem gut zu lokalisierenden Leuchtverhalten um $600 \mathrm{~nm}$ befasst, welches sich im Rahmen der Analyse auf Mangan zurückführen lässt (Kapitel 5.3). Zuletzt erfolgt die Analyse einer Auswahl von Europium-spezifischen Linien (Kapitel 5.4). In jedem Abschnitt wird sowohl auf die vermessenen zeitabhängigen Transienten als auch die zeitunabhängigen spektralen Eigenschaften der Proben eingegangen. Wie zu Beginn der Arbeit schon dargestellt dient AlN hierbei nur als genauer betrachtetes Beispielsystem. Generell lassen sich mit dem hier vorgestellten Lumineszenzaufbau eine Vielzahl verschiedener Materialsysteme untersuchen.

\subsection{Proben}

Im Gegensatz zu den in der Literatur typischerweise durch MBE, MOCVD, HVPE oder PVT hergestellten AlN-Proben (siehe dazu Kapitel 2) handelt es sich bei den hier verwendeten Präparaten um AlN, welches über ein Hochdruck-HochtemperaturVerfahren (engl. high-pressure, high-temperature, HPHT) mit der Temperaturgradienten-Methode von Taniguchi et al. (National Institute for Materials Science, Namiki 1 - 1, Tsukuba, Ibaraki 305-0044, Japan) synthetisiert worden ist. Als Ausgangsmaterial dient $\mathrm{Li}_{3} \mathrm{AlN}_{2}$-Pulver, welches im Fall von europiumdotierten Proben unter einer trockenen Stickstoffatmosphäre (zur Vermeidung der Oxidation der Ausgangsmaterialien) mit $\mathrm{EuF}_{3}$ vermengt wurde. Danach wurden die Mischungen in einer Molybdän-Kammer verschlossen und in einen HochdruckHochtemperaturapparat mit einem Bohrdurchmesser von $60 \mathrm{~mm}$ eingebracht 314. Für eine Dauer von bis zu 67 Stunden wurden die Ausgangsmaterialien einem Druck von $6.3 \mathrm{GPa}$ und einer Temperatur von maximal $1620^{\circ} \mathrm{C}$ ausgesetzt. Anschließend fand eine langsame Abkühlung zurück auf Raumtemperatur statt. Um die Proben aus der verpressten Molybdän-Kammer einfach zu extrahieren, wurde jene darauf- 
hin mit Königswasser aufgelöst. Bei den extrahierten Kristallen mit maximalen Größen kleiner als $0.5 \mathrm{~mm}$ handelt es sich Röntgenspektroskopiemessungen nach um AlN, welches in wurtzitischer Phase mit der Raumgruppe $C_{6 v}^{4}$ vorliegt [228]. Aluminium und Stickstoff besetzten dabei jeweils eine $C_{3 v}$-Position. Neben der wurtzitischen Phase wurden keine weiteren anderen Phasen gefunden. Auch gibt es keinerlei Hinweise auf eine Anreicherung von $E u^{3+}[228]$. Jenes liegt in den mit $\mathrm{EuF}_{3}$ vermischten Ausgangspräparaten in den Kristallen mit einer Konzentration von etwa 0.2 at. wt. $\dot{\%}$ vor. Eine spektroskopisch hoch aufgelöste Untersuchung der mit Europium dotierten Proben mit einer entsprechenden Zuordnung der zum Europium gehörigen Übergänge ist bei Gruber et al. 228 zu finden. Zuletzt durchgeführten RBS-Messungen (Rutherford-Rückstreu-Spektrometrie-Messungen) nach liegt die obere Grenze für eine chemische Verunreinigung durch schwere Elemente für die untersuchten Proben bei unter 0.1\%. Um die Präparate auf dem Probenhalter (siehe Abbildung 3.18(b)) anzubringen, wurde sich hier für Leitsilber als Bindemittel entschieden, da die Graphitpads selbst auch eine ausgeprägte optische Emission im hier relevanten spektralen Bereich aufweisen. Dieser mögliche zusätzliche, störende Untergrund kann so ausgeschlossen werden. Des weiteren ist mit Hilfe des Leitsilbers auch eine deutlich bessere thermische Kopplung durch ein teilweises Umschließen der Kristalle an den Kupferhalter gegeben. Diese umschließende Eigenschaft erweist sich jedoch für kleine Kristalle als Nachteil, da jene leicht komplett im Leitsilber versinken und damit nicht mehr für spektroskopische Experimente zur Verfügung stehen.

Zur besseren Vergleichbarkeit mit in der Literatur gegebenen Werten als auch der Möglichkeit zur Anpassung der Spektren mit typischen Verteilungsfunktionen werden die über die Wellenlängen aufgenommenen Spektren linear gegen die zugehörigen Energien aufgetragen. Aufgrund der linearen Intervallbreite der Pixeldetektoren beziehungsweise des Photomultipliers bezüglich der Wellenlänge und der Beziehung:

$$
E=\frac{h c}{\lambda}
$$

ergibt sich für die daraus folgenden gemessenen Intensitäten der Zusammenhang:

$$
I(E) \propto \lambda^{2} I(\lambda)
$$

Die hier gezeigten Spektren wurden durch die in den Abbildungen 4.4 beziehungsweise 3.19 dargestellten Sensitivitäten korrigiert. Diese Bereinigung der Spektren durch den Ausgleich der Effizienzen der einzelnen im Strahlengang und zur Messung des Signals vorhandenen Elemente ist nicht als vollständige Korrektur zu verstehen, da chromatische Abbildungsfehler durch das davor befindliche Linsensystem aus schon in Kapitel 4.2 benannten Gründen nicht vollständig berücksichtigt werden konnten. Zudem liegen für die Gitter nur Daten in der Littrow-Anordnung vor, welche durch eine Rotation der Gitter bezüglich des Eingangsspalts zur Auswahl unterschiedlicher 
Wellenlängenbereiche nicht mehr gegeben ist. Dennoch führt diese Anpassung zu einem realistischeren Bild der spektralen Emissionseigenschaften der Proben.

Für die zeitunabhängigen Messungen zur spektralen Analyse wurde stets das $1 \mathrm{~m}$-Spektrometer (siehe Kapitel 3.3.1) unter Zuhilfenahme des Photomultipliers verwendet, da jener im Gegensatz zum auch vorhandenen CCD keine Einschränkung bezüglich der in einer Messung gleichzeitig aufnehmbaren Energiebereiche aufweist und in seiner Effizienz gut dokumentiert ist. Zudem lässt er ein deutlich höheres Kontrastverhältnis zwischen geringen und hohen Intensitäten zu. Nachteilig hingegen erweist sich hier die Arbeit des Spektrometers als Monochromator, indem jeweils nur eine Wellenlänge allein analysiert werden kann. Dies erhöht die Messzeit eines Spektrums erheblich (>6 min), wodurch Drifts der Proben durch die Pumpenbewegungen und Intensitätsänderungen des Anregungssignals über die Zeit (siehe Kapitel 3.1.4) starke Einflüsse auf das zu vermessende Intensitätsspektrum haben. Zur Minderung solcher Effekte wurden diese Experimente in Serie bis zu 20 mal wiederholt und daraufhin gemittelt. Die in solchen Spektren angegebenen Fehler basieren auf der Bestimmung des Standardfehlers des Mittelwertes.

Dargestellt in Abbildung 5.1 sind zwei typische Übersichtsspektren bei Raumtemperatur der hier primär behandelten Proben. In den folgenden Abschnitten werden die einzelnen spektralen Bereiche als auch deren zeitliches Verhalten genauer untersucht.

\subsection{Defektlumineszenz}

Das Lumineszenzverhalten der hier untersuchten Proben wird durch die in den Kristallen vorhandenen Defekte dominiert. Über einen Bereich von $1.7 \mathrm{eV}$ bis hin zu $4.1 \mathrm{eV}$ sind unabhängig davon, ob die Präparate dotiert oder nicht dotiert sind breit verteilte Lumineszenzsignale zu beobachten (siehe Abbildung 5.1).

\subsubsection{Statisches Lumineszenzverhalten}

Für die spektrale Analyse wurden zunächst zeitunabhängige Aufnahmen für einen Temperaturbereich von $25 \mathrm{~K}$ bis $290 \mathrm{~K}$ angefertigt. Nach einer Subtraktion des Untergrunds wurden daraufhin Anpassungen der Form:

$$
I(E)=\sum_{i=1}^{4} \frac{I_{i}}{\sqrt{2 \pi \sigma_{i}^{2}}} \exp \left(-\frac{\left(E-E_{i}\right)^{2}}{2 \sigma_{i}^{2}}\right)
$$

vorgenommen. Für die breiten Lumineszenzbänder können zur Beschreibung des Verlaufs jeweils Normalverteilungen angenommen werden. Neben den energetischen Peak-Positionen $E_{i}$ sind auch die jeweiligen Standardabweichungen $\sigma_{i}$, sowie die Intensitäten $I_{i}$ zunächst als Parameter frei gewählt. Dies führt jedoch mit steigender 


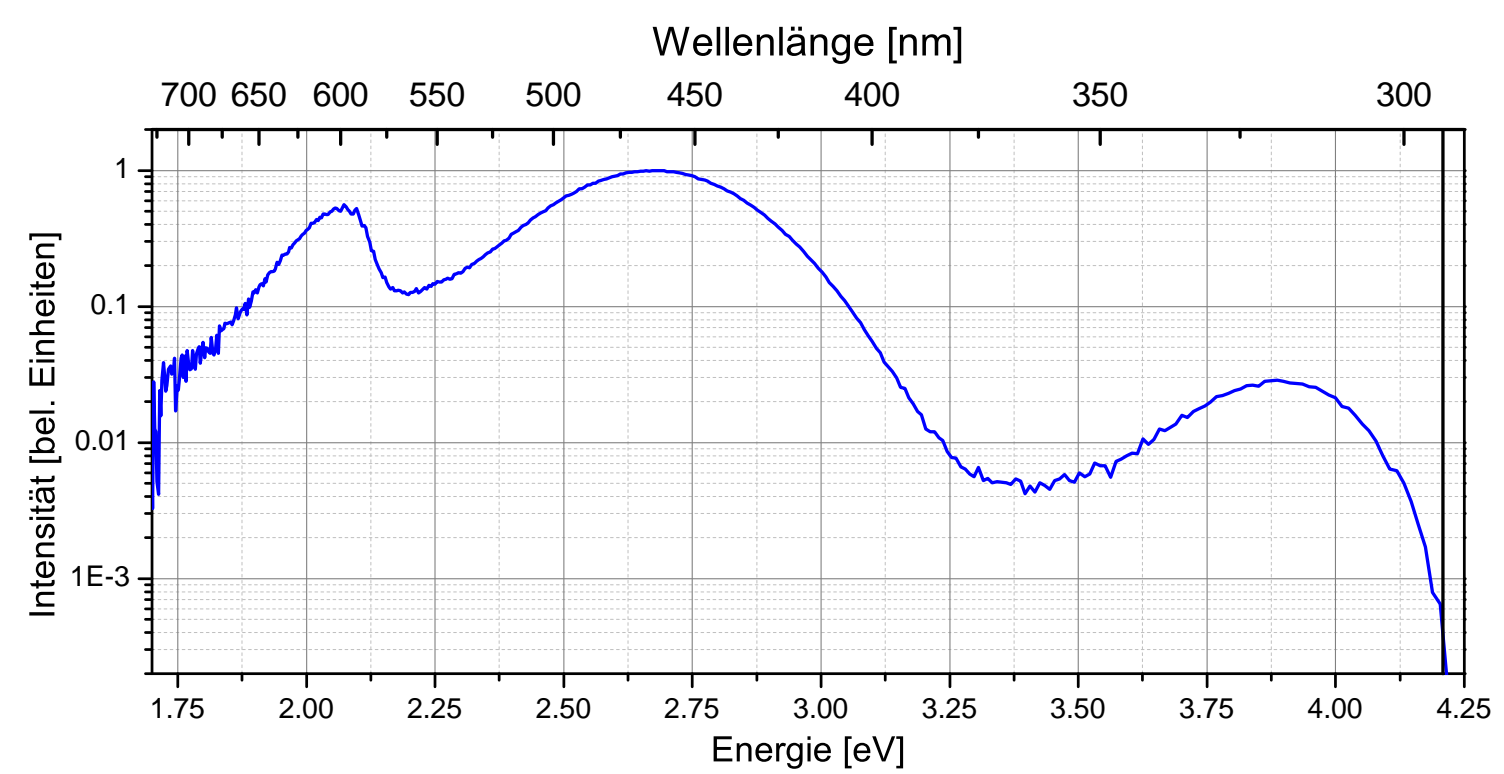

(a) Undotiertes AlN

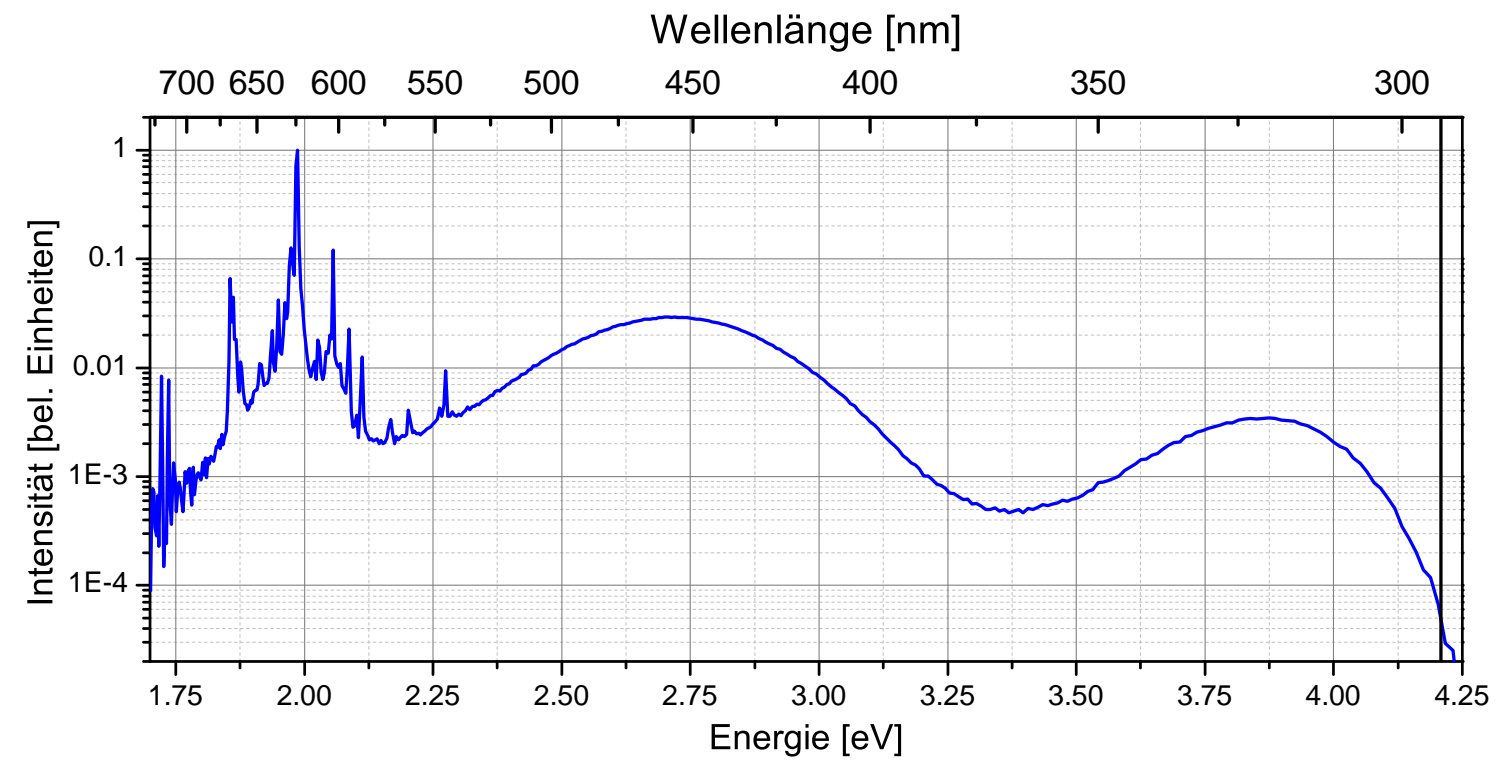

(b) $E u^{3+}$ dotiertes $\mathrm{AlN}$

Abbildung 5.1: Übersichtsspektren einer undotierten (oben) und einer mit $E u^{3+}$ dotierten AlN-Probe (unten) aufgenommen mit dem $1 \mathrm{~m}$-Spektrometer, einem $100 \mathrm{~g} / \mathrm{mm}$-Gitter und dem Photomultiplier R928 bei Raumtemperatur. Zur Unterdrückung des Lasersignals (THG, $\lambda_{e x}=267 \mathrm{~nm}$ ) befindet sich ein Tiefpassfilter $\left(\lambda_{\text {cut }}=295 \mathrm{~nm}\right)$ im Strahlengang (schwarze senkrechte Linie). Das Messsignal wurde nach der in Abbildung 3.19 dargestellten Effizienz korrigiert. 
Temperatur zu physikalisch nicht sinnvollen Lösungen durch einen Drift einer Normalverteilung hin zu einem Median um $1 \mathrm{eV}$ mit hohen Intensitäten in Bereichen negativer Emissionsenergie; hervorgerufen durch die Symmetrie der Verteilung und eine große Standardabweichung. Zur Stabilisierung der Fits wurde als Randbedingung daher eine Intensität von 0 (realisiert als $10^{-30}$ ) bei der Position $0 \mathrm{eV}$ eingeführt. Die resultierenden Anpassungen besitzen dabei ein reduziertes $\chi^{2}$ zwischen 0.3 und 1, was diesen Schritt rechtfertigen kann. In Abbildung 5.2 sind beispielhaft für jeweils zwei Temperaturen die Ergebnisse der Anpassungen an die Probensysteme grafisch dargestellt. Qualitativ zeigen die undotierten zu den dotierten Proben ein gleichartiges Spektrum, welches durch die vier Normalverteilungen gut beschrieben werden kann. Bei den Anpassungen bleiben die Signale unterhalb von $2.3 \mathrm{eV}$ unberücksichtigt, da jene später (siehe Kapitel 5.3 und 5.4 separat untersucht werden. Auch für Signale oberhalb von $4 \mathrm{eV}$ findet keine Berücksichtigung statt, da aufgrund des im Strahlengang bei den Messungen eingesetzten Langpassfilters $\left(\lambda_{\text {cut }}=295 \mathrm{~nm}\right)$ Signale im tiefen UV-Bereich nicht detektiert werden können und auch schon durch eine endliche Güte des Filters kurz nach $4 \mathrm{eV}$ eine Abschwächung erfahren.

Dominiert wird das Spektrum von einem breiten Band um $2.7 \mathrm{eV}$ (in Abbildung 5.2 jeweils mit " 2 " bezeichnet). In Abbildung 5.3 (a) sind die Halbwertsbreiten aus den Anpassungen nach Gleichung 5.3 dargestellt. Dabei fallen in ihrer Größe sehr unterschiedliche Unsicherheiten bei den ermittelten Werten für die verschiedenen Temperaturen auf. Die berechneten Fehler unterhalb von $225 \mathrm{~K}$ liegen in etwa eine Größenordnung über denen ab dieser Temperatur. Insgesamt zeigen sich für die zwei Probensysteme in ihrer Größe und Entwicklung sehr ähnliche Verläufe. Für solch breitbandige Übergänge kann eine Änderung der Halbwertsbreite nach 315 folgenden Verlauf annehmen:

$$
\gamma(T)=\gamma(0) \sqrt{\operatorname{coth}\left(\frac{E_{a}}{k_{\mathrm{B}} T}\right)} .
$$

Unter Berücksichtigung der ermittelten Unsicherheiten lassen sich durch einen Fit jeweils Aktivierungsenergien von $E_{a}=39.8(20) \mathrm{meV}(\mathrm{AlN})$ und $E_{a}=29.0(18) \mathrm{meV}$ $(\mathrm{AlN}+\mathrm{Eu})$ bestimmen. Besonders im Fall des undotierten AlN zeigt sich dabei jedoch eine starke Überschätzung der ermittelten Werte bei niedrigen Temperaturen. Bei einer Betrachtung der Streuung der Parameter im Bereich hoher Temperaturen scheint der dort angegebene Fehler als deutlich zu gering abgeschätzt. Deshalb wurde die gleiche Anpassung noch einmal bei einer Gleichgewichtung aller Werte ausgeführt, was Aktivierungsenergien von $E_{a}=30.9(12) \mathrm{meV}(\mathrm{AlN})$ und $E_{a}=29.4(21) \mathrm{meV}$ $(\mathrm{AlN}+\mathrm{Eu})$ liefert. Während sich der Fit beim dotierten AlN nur unwesentlich ändert, zeigt sich für das undotierte Präparat eine weitaus bessere Beschreibung der Daten.

Da insbesondere bei niedrigen Temperaturen unter der Annahme von vier Normalverteilungen auch signifikante Anteile der Intensität um $2.7 \mathrm{eV}$ von den zwei benachbarten Peaks (in der Abbildung 5.2 mit " 1 " und " 3 " bezeichnet) herrühren, 


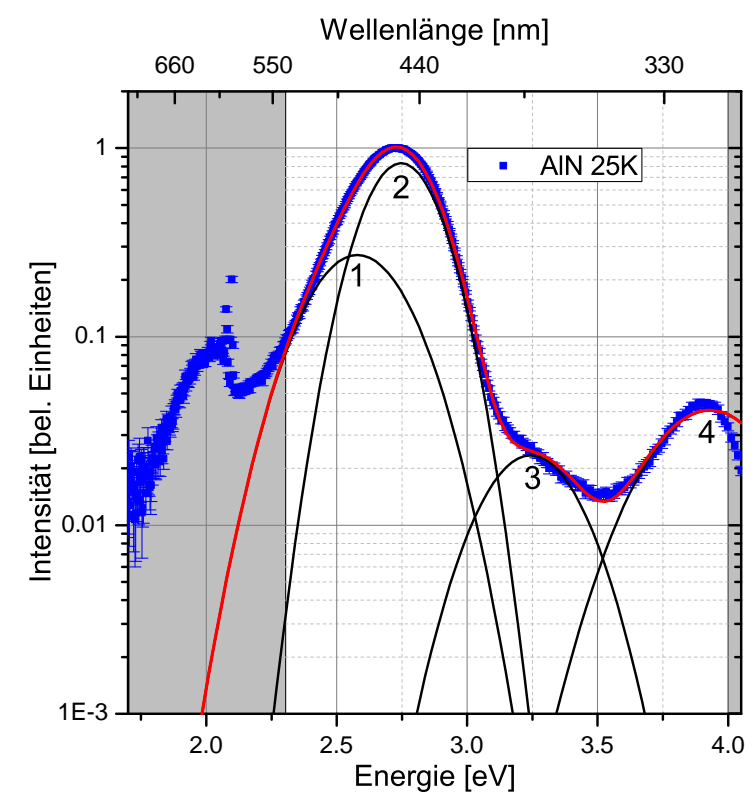

(a)

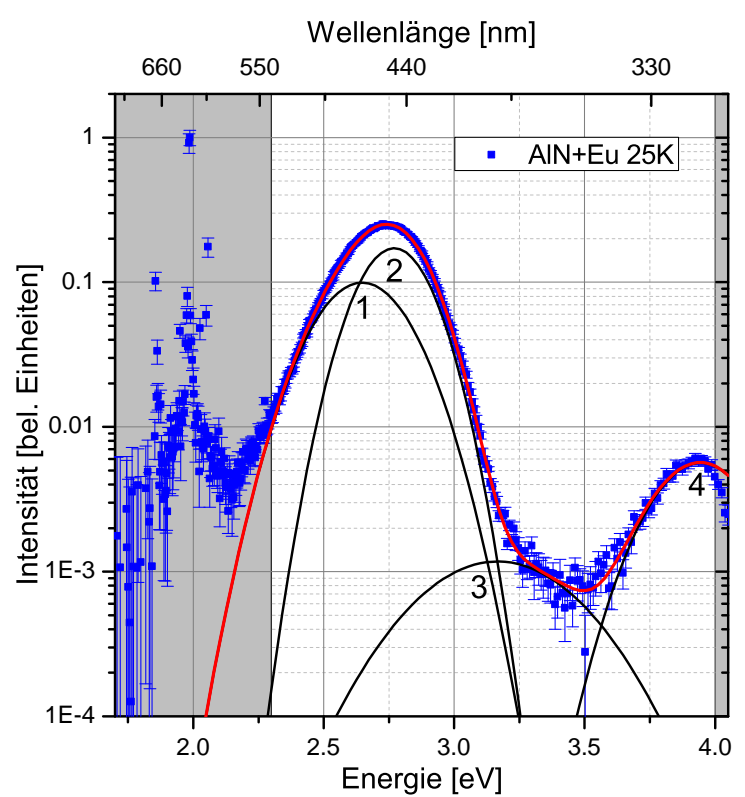

(c)

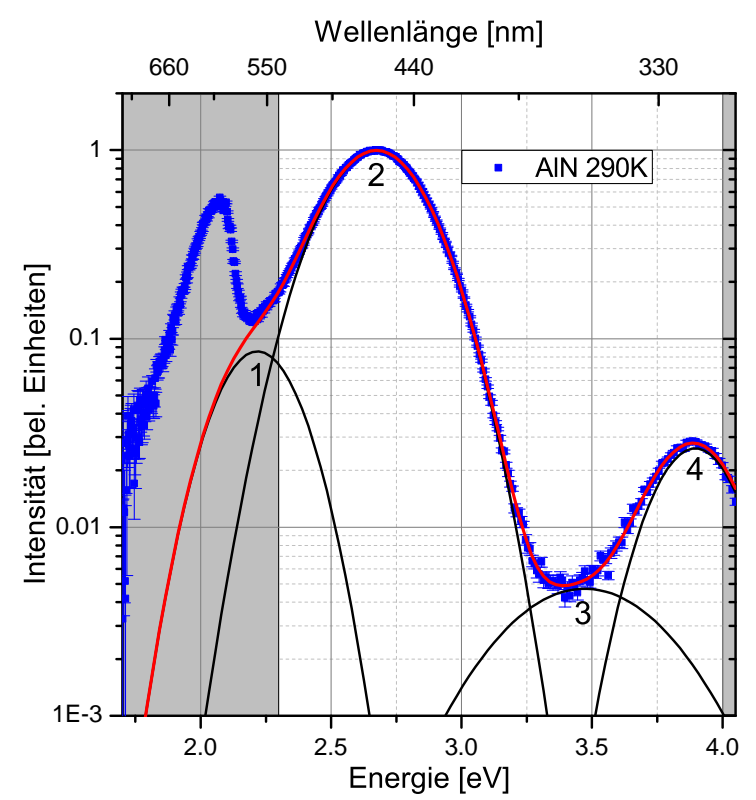

(b)

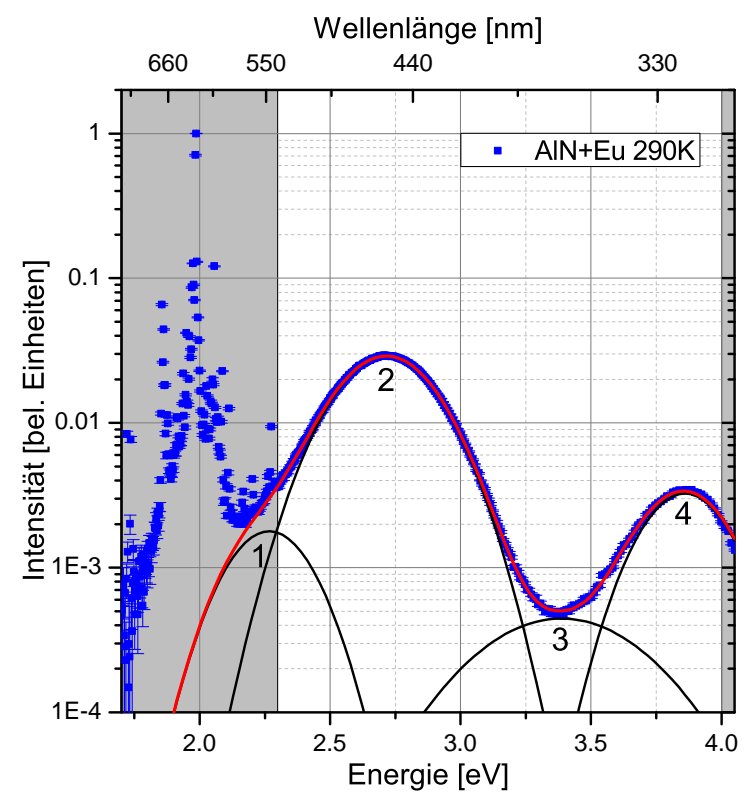

(d)

Abbildung 5.2: Anpassung der Defektlumineszenzen im undotiertem und mit Europium dotiertem AlN für jeweils zwei Temperaturen nach Gleichung 5.3 (rote Linien). Der jeweilig grau hinterlegte Bereich bleibt bei der Anpassung ohne Berücksichtigung. Mit Ausnahme von (a) zeigen sich mit den Daten gut übereinstimmende Kurvenverläufe (reduziertes $\chi^{2}<1$ ). 


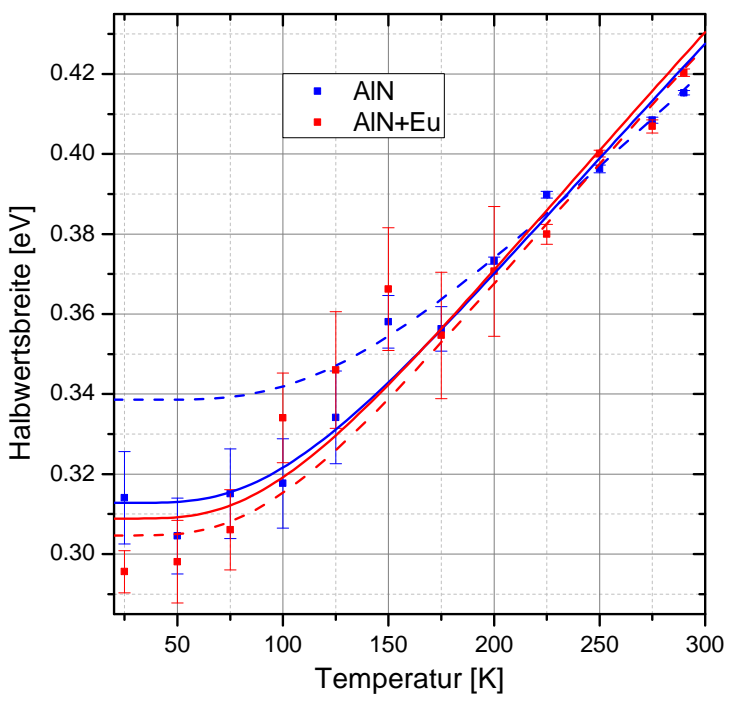

(a)

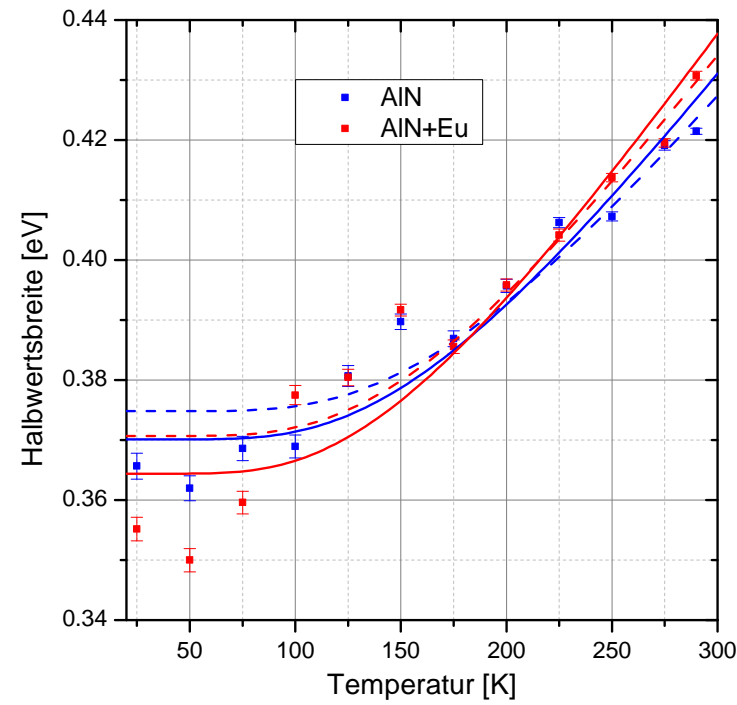

(b)

Abbildung 5.3: Temperaturabhängigkeit der Halbwertsbreite der Lumineszenz um $2.7 \mathrm{eV}$ für die zwei untersuchten Probensysteme. Die Linien kennzeichnen jeweilige Fits nach Gleichung 5.4 unter Berücksichtigung (gestrichelt) oder Missachtung (durchgezogen) der gegebenen Unsicherheiten. (a) Halbwertsbreite bestimmt aus der zweiten Normalverteilung der Anpassung nach Gleichung 5.3. (b) Halbwertsbreite bestimmt aus separater Anpassung des Messsignals mit einfacher Normalverteilung nach Gleichung 5.5 im Bereich von $2.4 \mathrm{eV}$ bis $3.1 \mathrm{eV}$.

wurde noch eine alternative Beschreibung des gesamten Maximums als einzelne Normalverteilung der Gestalt:

$$
I_{N}(E)=\frac{I_{N 0}}{\sqrt{2 \pi \sigma^{2}}} \exp \left(-\frac{\left(E-E_{N}\right)^{2}}{2 \sigma^{2}}\right)
$$

in Betracht gezogen. Dabei wurde nur ein eingeschränkter Bereich der Messdaten von $2.4 \mathrm{eV}$ bis $3.1 \mathrm{eV}$ in der Anpassung berücksichtigt. Die Ergebnisse für die daraus resultierenden Halbwertsbreiten sind in Abbildung 5.3 (b) dargestellt und weisen aufgrund der Zusammenfassung des Signals als eine einzelne Normalverteilung für niedrige Temperaturen verglichen zu der vorherigen Bestimmung etwa $20 \%$ größere Werte auf. Wie auch schon bei der vorherigen Methode zeigen die Datenpunkte für niedrige Temperaturen weitaus größere Unsicherheiten. Deshalb wurden auch in jenen Datensätzen jeweils zwei Anpassungen nach Gleichung 5.4 einmal unter einer Berücksichtigung und einmal unter einer Vernachlässigung der Fehler durchgeführt. Dabei ergeben sich für die Aktivierungsenergien jeweils Werte von $E_{a}=52.6(22) \mathrm{meV}$ $(\mathrm{AlN})$ und $E_{a}=47.9(25) \mathrm{meV}(\mathrm{AlN}+\mathrm{Eu})$; beziehungsweise unter Vernachlässigung der Unsicherheiten $E_{a}=48.8(21) \mathrm{meV}(\mathrm{AlN})$ und $E_{a}=44.2(26) \mathrm{meV}(\mathrm{AlN}+\mathrm{Eu})$. 
Neben der Halbwertsbreite ist auch eine Untersuchung der Verschiebung der PeakMaxima möglich. Nach Shionoya et al. 315] kann diese in den hier betrachteten Fällen durch eine lineare Verschiebung mit sich ändernder Temperatur beschrieben werden:

$$
E(T)=\left[\frac{\nu_{g}^{2}-\nu_{e}^{2}}{\nu_{e}^{2}}+\frac{8 \nu_{g}^{4}}{\nu_{e}^{2}\left(\nu_{g}^{2}+\nu_{e}^{2}\right)} \cdot \frac{E_{a b}(0)-E(0)}{E(0)}\right] \cdot k_{\mathrm{B}} T+E(0) .
$$

Der Vorfaktor bestehend aus vibronischen Frequenzen des Grundzustandes $\nu_{g}$ und des angeregten Zustandes $\nu_{e}$ (siehe Kapitel 3.1.2), sowie der Absorptions- $E_{a b}(0)$ und Emissionsenergie $E(0)$ des Maximums beim Temperaturnullpunkt kann aufgrund seiner Komplexität und Unbestimmtheit der Parameter lediglich als eine Konstante zusammengefasst werden. Die jeweiligen Verschiebungen der Maxima des Hauptpeaks innerhalb der Probensysteme sind für die zwei unterschiedlichen weiter oben vorgestellten Methoden in der Abbildung 5.4 dargestellt. Verglichen zur Verschiebung resultierend aus lediglich einer Normalverteilung zeigen die beiden Anpassungen aus der ersten Methode einen in ihrer Steigung ähnlicheren Wert. Die Werte bezüglich der ermittelten Unsicherheiten sind auch hier für die verschiedenen Temperaturbereiche höchst unterschiedlich, führen jedoch zu einer adäquaten Anpassung über den kompletten betrachteten Bereich. Insgesamt erscheinen die ermittelten Fehler bei der Anpassung lediglich einer Normalverteilung trotz einer konzeptionell guten Beschreibung des Verlaufs verglichen zu deren Streuung dennoch als zu klein angegeben.

Unabhängig vom dominierenden Peak bei $2.7 \mathrm{eV}$ lässt sich relativ weit separiert davon auch ein Lumineszenzzentrum um $3.9 \mathrm{eV}$ (in Abbildung 5.2 jeweils mit " 4 " bezeichnet) beobachten. Die ermittelten zugehörigen Halbwertsbreiten sowie Positionen sind in Abbildung 5.5 dargestellt. Aufgrund des während der Messung notwendigerweise eingesetzten Filters zur Unterdrückung der anregenden Wellenlänge, kann lediglich der niederenergetische Teil dieses Übergangs vollständig vermessen werden. Dies führt zu sehr großen Unsicherheiten bei den ermittelten Werten, weshalb auf eine Anpassung verschiedener theoretischer Modelle in diesen Fällen verzichtet wurde. Die Halbwertsbreite scheint demnach mit steigender Temperatur zumindest konstant, wenn nicht sogar im Fall des undotierten AlN zu sinken, was durch einen einzelnen Übergang nicht ohne weiteres erklärt werden kann. Konsistent für beide Probensysteme sinkt auch die energetische Position des Übergangsmaximums, jedoch auch hier unter hoher statistischer Streuung.

Eine relative Intensitätsbestimmung einzelner Linien ist aufgrund des schrägen Lasereinfalls und einer damit einhergehenden lateralen Verschiebung der Leuchtzentren für verschiedene Temperaturen und einer somit notwendigen Nachjustage der Eingangsoptik nicht ohne weiteres realisierbar. Jedoch ergibt sich die Möglichkeit, bei einer konstant gehaltenen Fokusebene die Intensitäten der verschiedenen Linien untereinander zu vergleichen. Eine auf die Intensität des stärksten Lumineszenzsignals 


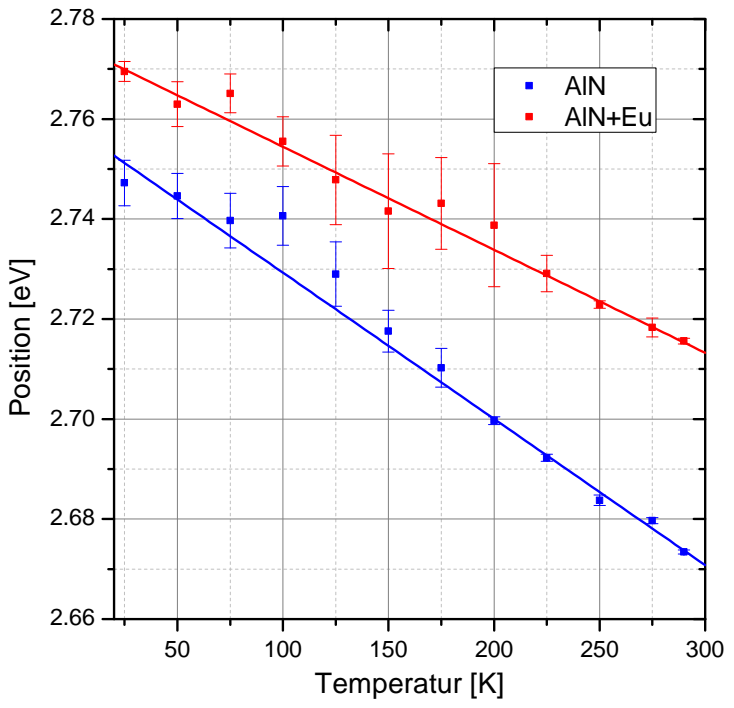

(a)

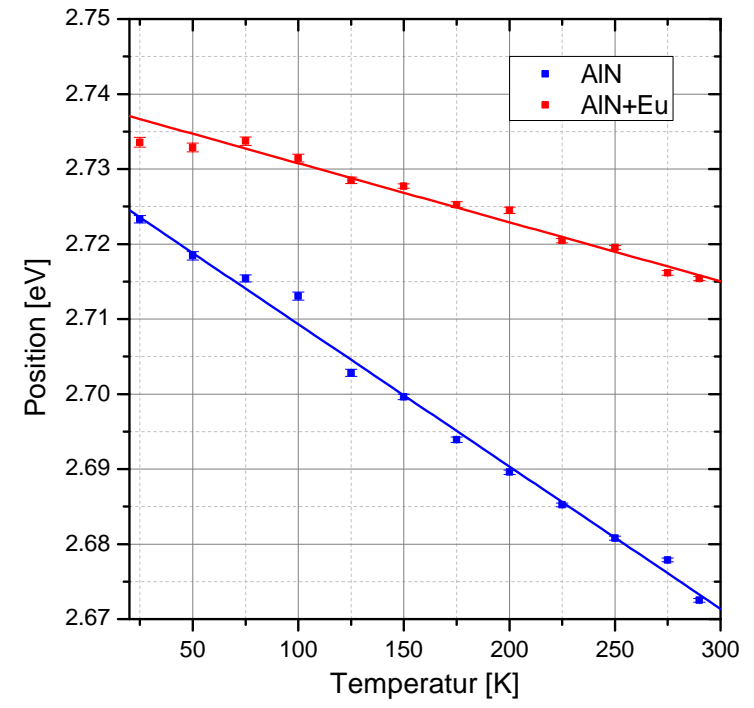

(b)

Abbildung 5.4: Temperaturabhängigkeit der energetischen Peak-Position der Lumineszenz um $2.7 \mathrm{eV}$ für die zwei untersuchten Probensysteme. Die Daten stammen, äquivalent zu Abbildung 5.3, aus den Anpassungen nach: (a) Gleichung 5.3 beziehungsweise (b) Gleichung 5.5. Bei den Linien handelt es sich um jeweilige Fits der Gleichung 5.6 .

um $2.7 \mathrm{eV}$ normierte Darstellung der Intensitäten der drei weiteren Nebenbänder ist in Abbildung 5.6 gegeben. Für die zwei betrachteten Probensysteme zeigen sich bei der integrierten Intensität der Normalverteilung "1" sowohl in der Richtung als auch in der Größenordnung gleiche abfallende Verläufe. Im Gegensatz dazu sinkt die relative Intensität der zwei verbleibenden Bänder im Verhältnis zum Hauptpeak im Fall des undotierten AlN mit steigender Temperatur stetig ab, während sie sich im europiumdotiertem Präparat relativ stabil mit einer sogar leicht steigenden Tendenz über den betrachteten Temperaturbereich entwickeln.

\section{Diskussion der zeitunabhängigen Spektren}

Die Beschreibung der breitbandigen Lumineszenzen im sichtbaren und nahen UVBereich in den zwei betrachteten Probensystemen durch vier Normalverteilungen unter Einführung einer zusätzlichen Randbedingung liefert eine gute Repräsentation der Daten mit einem reduzierten $\chi^{2}$ in einer Größenordnung des aus den Messungen resultierenden Fehlers. Jedoch zeigen sich für niedrige gegenüber hohen Temperaturen in den ermittelten Halbwertsbreiten, Positionen als auch den integrierten Intensitäten deutlich größere Unsicherheiten. Eine Möglichkeit zur Erklärung könnten um eine Größenordnung unterschiedliche absolute Zählraten bei verschiede- 


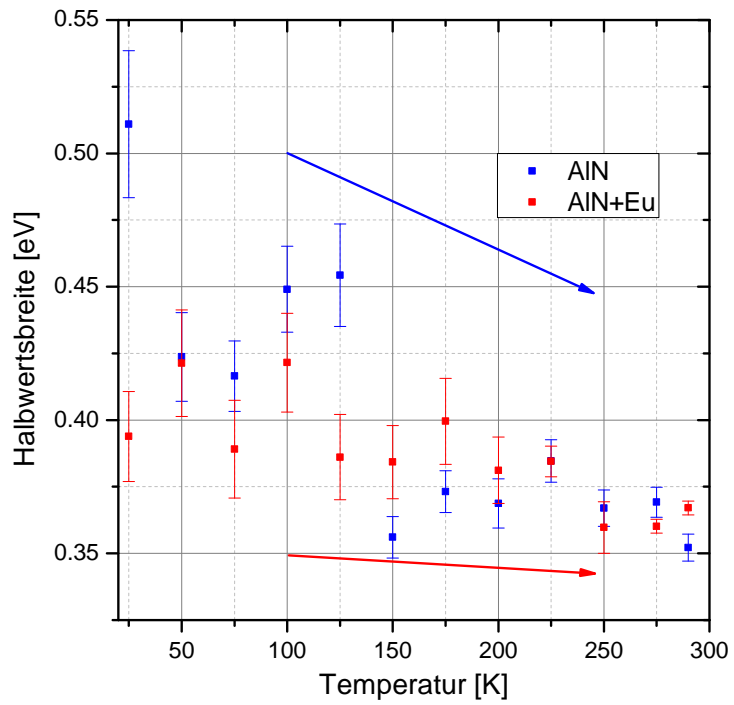

(a)

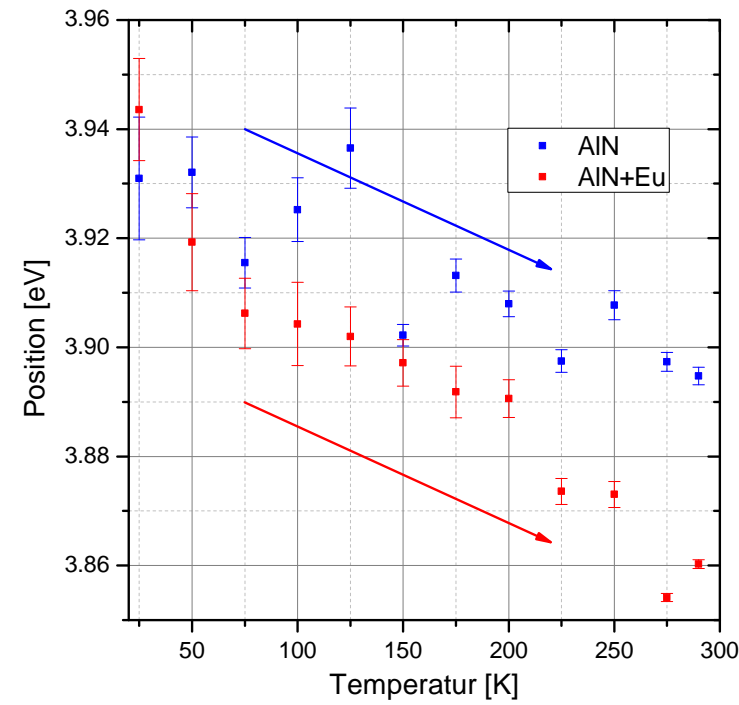

(b)

Abbildung 5.5: (a) Halbwertsbreite beziehungsweise (b) Position der Lumineszenz um $3.9 \mathrm{eV}$ in Abhängigkeit zur Temperatur. Auf eine Anpassung nach verschiedenen Modellen wurde aufgrund der hohen Streuung beziehungsweise des unspezifischen Verlaufs, hervorgerufen durch eine nicht mögliche vollständige Erfassung des Lumineszenzsignals, verzichtet. Die Pfeile deuten die jeweiligen Tendenzen mit zunehmender Temperatur an.

nen Temperaturen, welche dann auch die Größe der jeweiligen relativen Fehler der Messdaten beeinflussen würden, sein. Die relativen Abweichungen zeigen bezüglich der Intensitäten jedoch keine dahingehenden Unterschiede um solch große Diskrepanzen zu erklären. Eine andere Ursache könnte die während einer Messung sich über die Zeit ändernde Anregungsintensität (siehe Kapitel 3.1.4) in Verbindung mit einer leichten Modulation der Position der Proben durch die Kühlpumpe (siehe Kapitel 3.2) darstellen, welche durch die Mittlung von jeweils 20 Messreihen einen starken Einfluss auf die statistischen Schwankungen der Messdaten haben. Bei gleichen prozentualen Schwankungen ergeben sich so unabhängig von den absolut gemessenen Zählraten ähnlich große relative Fehler innerhalb der Daten. Da vor allem der niederenergetischste Peak (in Abbildung 5.6 als "1" bezeichnet) verglichen zum dominierenden Signal bei $2.7 \mathrm{eV}$ im absoluten Verhältnis den größten Intensitätsverlust mit steigender Temperatur aufweist, kann dies zu einer stärkeren Entkopplung der Parameter und einem damit einhergehenden Absinken der ermittelten Unsicherheiten führen. Eine Unberücksichtigung der Fehler und eine somit Gleichgewichtung aller aus den Fits resultierenden Parameter führt über die Probensysteme hinweg zudem zu einem konsistenteren Ergebnis der bestimmten Aktivierungsenergien. Eine Beschreibung des Hauptpeaks als lediglich eine einzelne Normalverteilung, erweist 


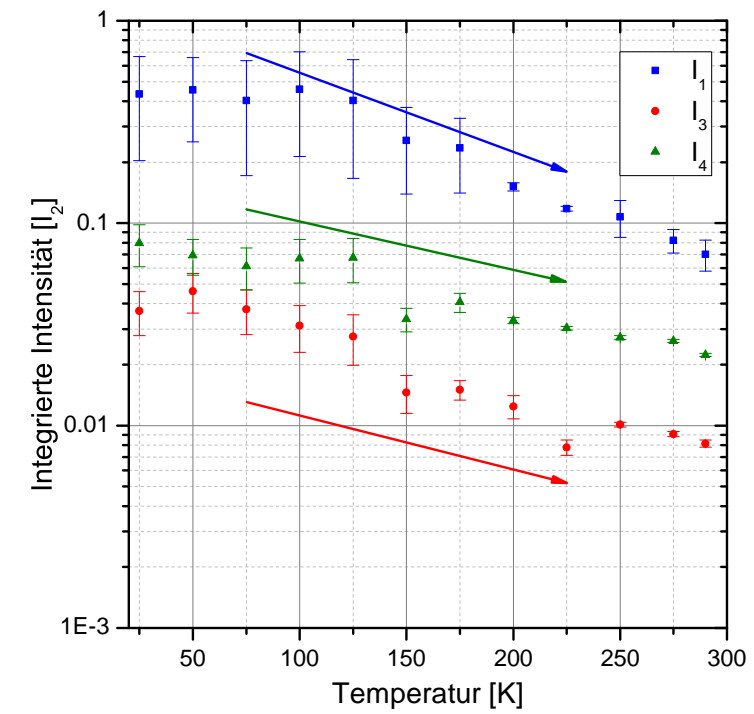

(a) AlN

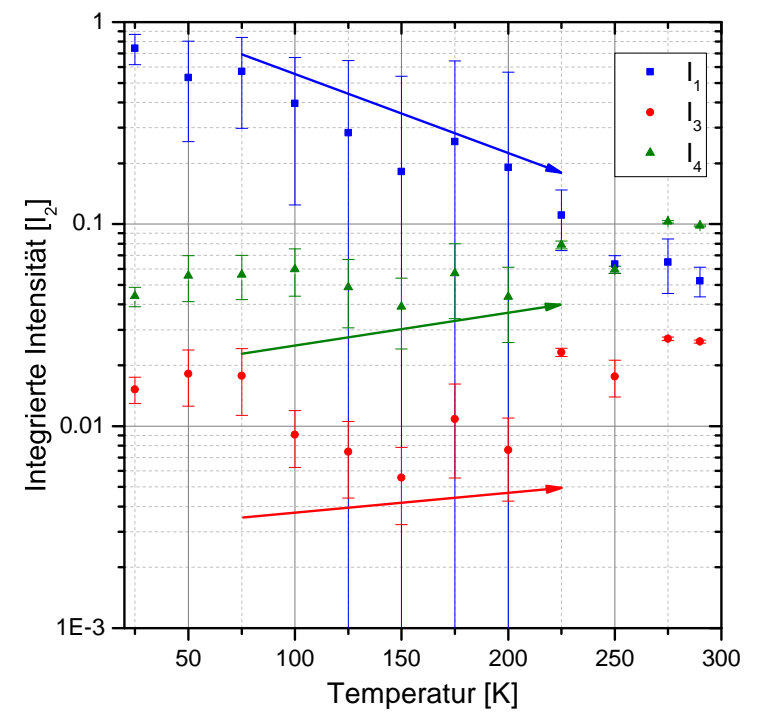

(b) $\mathrm{AlN}+\mathrm{Eu}$

Abbildung 5.6: Intensitäten der drei schwächeren normalverteilten Bänder aus den Anpassungen nach Gleichung 5.3 normiert auf das stärkste Band. Die Fehlerbalken ergeben sich durch die Unsicherheiten der berechneten Parameter während der durchgeführten Anpassungen. Die Pfeile deuten die jeweiligen Tendenzen mit zunehmender Temperatur an.

sich für die zwei Probensysteme als weniger konsistent. Während sich die berechneten Maximaverschiebungen bei der ersteren Methode nur um 29.6\% unterscheiden, liegt die Diskrepanz bei einer Beschreibung durch lediglich eine Normalverteilung bei 58.6\% . Die ermittelten Aktivierungsenergien aus den Halbwertsbreiten liegen in etwa $30 \%$ über denen aus einer Anpassung aus vier Normalverteilungen. Dabei zeigt sich zudem, dass eine Peakbeschreibung nur unmittelbar um das Maximum selbst durch eine einzelne Verteilung abgebildet werden kann und sich nach außen hin wesentliche Diskrepanzen in Bezug auf die Messdaten ergeben. Dies wird in der anderen Methode mit Hilfe der umliegenden Nebenbänder kompensiert.

Die beobachtete Tendenz einer sinkenden Halbwertsbreite mit steigender Temperatur für das Signal um $3.9 \mathrm{eV}$ wäre für einen einzelnen Prozess sehr ungewöhnlich. Eine mögliche Erklärung könnte ein zweiter in dieser Region vorhandener Transfer sein, welcher mit steigender Temperatur in seiner Intensität absinkt; beispielsweise hervorgerufen durch einen strahlungslosen Übergang in seinen Grundzustand oder einen Transfer in ein anderes Defektniveau. Des weiteren ergibt sich zudem aufgrund der lediglich halbseitigen Erfassung bedingt durch die Messmethodik eine relativ große Unsicherheit bezüglich der bestimmten Halbwertsbreiten als auch Maxima.

Die Unterschiede in den relativen Intensitätsverläufen bezüglich des dominierenden Defektsignals bei $2.7 \mathrm{eV}$ in den zwei betrachteten Probensystemen könnte 
infolge einer vermehrten Löschung einiger Lumineszenzanteile durch das aktivierte Europium im dotierten AlN hervorgerufen werden. Im undotierten Fall sinken alle Intensitäten verglichen zum Hauptpeak mit steigender Temperatur stark ab, während im dotierten Präparat lediglich das niederenergetischste Signal noch an Intensität verliert. Dieses Verhalten könnte durch eine mit zunehmender Temperatur anwachsende Übertragung der Emissionsenergie der beiden Haupttransfers an das im AlN befindliche Europiumsystem erklärt werden, da jenes in seiner Intensität in gleicher Weise stark ansteigt.

Nur aus den zeitunabhängigen Spektren lässt sich eine genauere Zuordnung der beobachteten Übergänge zu in AlN bekannten Defektsystemen (siehe Kapitel 2.2 aufgrund der Vielzahl der möglichen Ursachen nur schwer treffen. Daher wurden neben den statischen Aufnahmen auch zeitabhängige Spektren für die zwei Probensysteme analysiert, wodurch eine leichtere Separation ermöglicht wird.

\subsubsection{Zeitabhängiges Lumineszenzverhalten}

Messungen des zeitabhängigen Verhaltens der breitbandigen Lumineszenzsignale können zum einen einen Aufschluss über den generellen Ursprung eines Defektsignals (siehe Kapitel 2.2) als auch eine durch beispielsweise unterschiedliche zeitliche Skalen hervorgerufene Separation sich ansonsten überlagernder Bänder ermöglichen.

Wie in Abbildung 5.7(a) zu sehen, handelt es sich bei der Lumineszenz um $3.9 \mathrm{eV}$ um ein sehr kurzlebiges Signal. Die aus einer nach Gleichung A.3 ermittelten Lebensdauern (siehe Abbildung 5.7(b)) liegen dabei im Bereich der zeitlichen Auflösung des hier verwendeten spektroskopischen Aufbaus (siehe Kapitel 3.3.2). Bedingt durch eine in der Aufnahme hohe erzielte Statistik ist es dennoch möglich, Aussagen über das zeitliche Verhalten dieses Signals zu treffen. Bei Messungen mit solch kurzen Zeitfenstern spielt das Wandern des relativen Trigger-Zeitpunktes eine große Rolle bei der Erfassung der Daten (siehe Kapitel 4.3). Auch das zur Auswertung verwendete Modell zur Beschreibung jener hat einen signifikanten Einfluss. Um einen möglichst hohen als auch präzisen Informationsgewinn aus den Daten erhalten zu können, wurden jene deshalb zuvor in ihrer über die Messzeit auftretenden Verschiebungen korrigiert und Anpassungen unter Berücksichtigung der zeitlichen Aufösung durchgeführt. Die Aufösung wurde dabei für jede Temperatur als neuer freier Parameter zugelassen, da diese innerhalb der durchgeführten Messreihen leicht variieren kann. Dabei zeigen sich mit steigender Temperatur für beide betrachteten Systeme um lediglich etwa 3\% reduzierte Lebensdauern, was aufgrund der von der Streak-Einheit her vorgegebenen im Vergleich dazu großen zeitlichen Auflösung zu relativ hohen Unsicherheiten bei den ermittelten Zeiten als auch den daraus berechneten Aktivierungsenergien führt.

Auch für die Signale um $2.7 \mathrm{eV}$ existiert ein kurzlebiger Anteil mit Lebensdauern im Bereich weniger Nanosekunden und darunter. Die dort ermittelten Intensitäten wurden ebenfalls wie bei der Lumineszenz um $3.9 \mathrm{eV}$ nach Gleichung A.3 ange- 


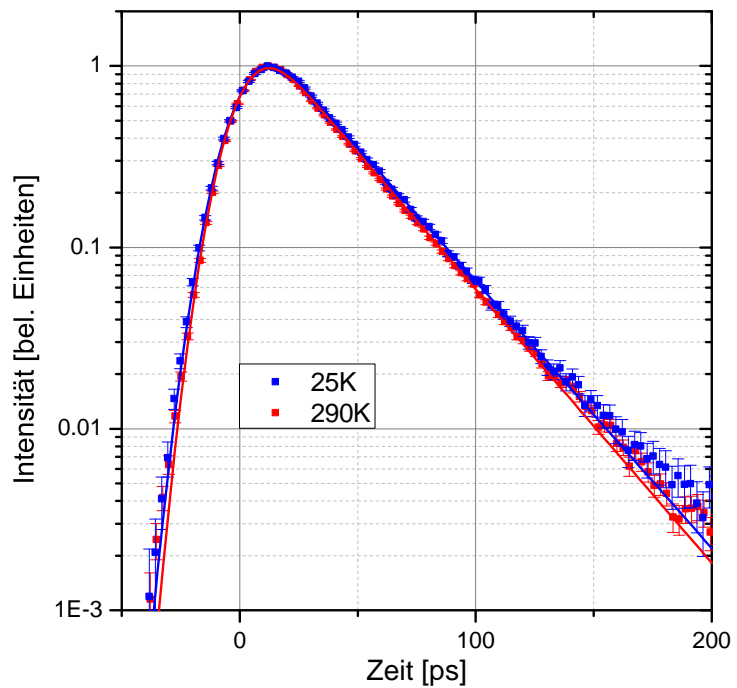

(a)

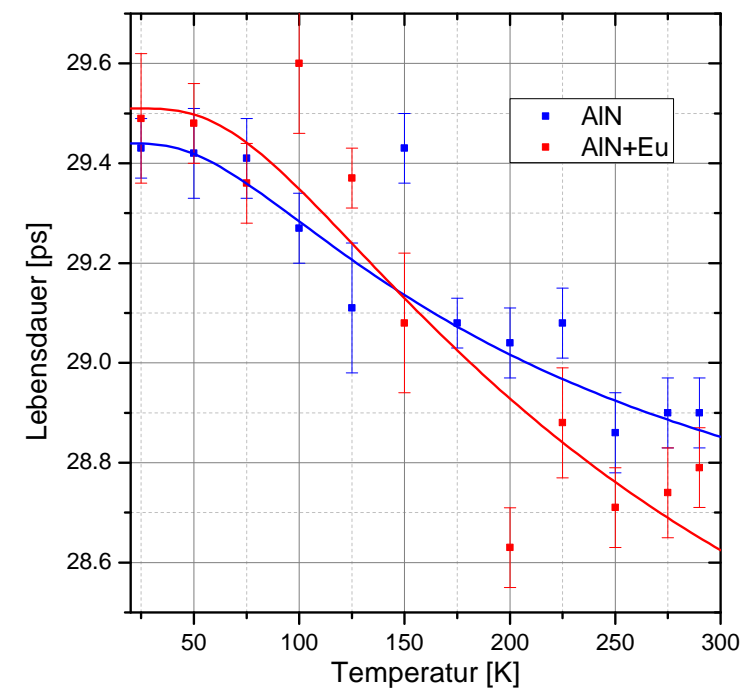

(b)

Abbildung 5.7: (a) Zeitliches Verhalten der Lebensdauer um $3.9 \mathrm{eV}$ in europiumdotiertem AlN. Bei den Linien handelt es sich um Anpassungen nach Gleichung A.3. Der Untergrund wurde zuvor schon vom Signal subtrahiert und liegt in der Größenordnung um $10^{-3}$. (b) Aus den Fits bestimmte Lebensdauern für beide Materialsysteme. Bei den Linien handelt es sich um Anpassungen nach Gleichung 5.20 mit resultierenden Aktivierungsenergien von jeweils $E_{a}=17(6) \mathrm{meV}$ (blau) und $E_{a}=22(10) \mathrm{meV}$ (rot).

passt, wobei es sich hierbei um ein Aufnahmezeitfenster von 5 ns handelte. Zudem wurden zwei voneinander unabhängige Rekombinationsprozesse mit jeweils eigenen Lebensdauern zugrunde gelegt. Die aus den Fits resultierenden Parameter sind in Abbildung 5.8 dargestellt. Dabei zeigt sich zum einen ein Transfer mit einer Lebensdauer im Bereich von 1 - 2 ns als auch ein weiterer Prozess mit einer erheblich schnellerer Rekombinationsrate. Der langsamere der beiden Prozesse besitzt im undotierten AlN eine Temperaturabhängigkeit, welche über einen Mott-Seitz Ansatz 316] nach Gleichung 5.20 beschrieben werden kann, während die Lebensdauer im europiumdotiertem Präparat über den gesamten Temperaturbereich konstant erscheint. Der kurzlebige Anteil liegt in seiner Rekombinationsrate über der in diesem Zeitfenster zu erreichenden Auflösung. Auch eine Messung mit einem kürzeren Zeitfenster zeigt, dass sich die Lebensdauer in der Größenordnung der erreichbaren Aufösung des verwendeten Systems oder sogar darunter befindet. Im Gegensatz zum Signal bei $3.9 \mathrm{eV}$ besitzt die kurzlebige Lumineszenz um $2.7 \mathrm{eV}$ einen signifikanten langlebigen Untergrund, welcher eine genauere Analyse hervorgerufen durch die statistische Streuung jenes deutlich erschwert und dadurch sehr lange Messzeiten für jede einzelne Temperatur verlangen würde. 


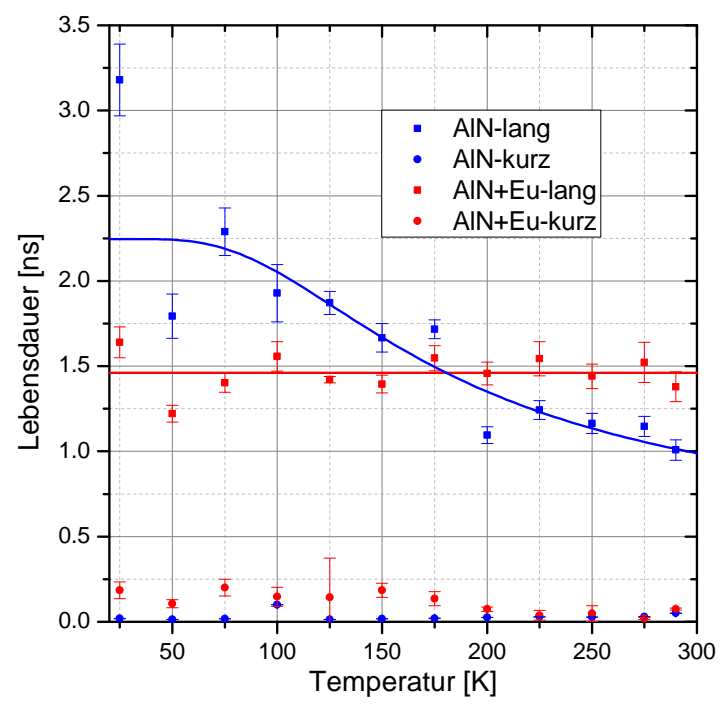

Abbildung 5.8: Lebensdauern des Kurzlebigen Anteils um $2.7 \mathrm{eV}$ für die zwei betrachteten Systeme aufgetragen gegen die Temperatur. Die Lebensdauern wurden aus einer Anpassung nach Gleichung A.3 für zwei unabhängige Zerfallskanäle bestimmt. Die Fits zeigen jeweils eine Anpassung nach Gleichung 5.20 (blaue Linie) beziehungsweise den gewichteten Mittelwert (rote Linie).

Dieser Signaluntergrund liegt in seiner Lebensdauer um mehrere Größenordnungen über denen der kurzlebigen Übergänge. Zur besseren Veranschaulichung sind hierfür jeweils eine Messung mit einem sich öffnenden und sich schließenden Fenster des Chopperrads in Abbildung 5.9 für den sichtbaren Wellenlängenbereich dargestellt. Dabei sind für die Lumineszenz um $2.7 \mathrm{eV}$ deutliche Unterschiede während der Anregung und danach auszumachen. Der kurzlebige Anteil zeichnet sich durch einen abrupten Wechsel der Intensität beim Öffnen beziehungsweise Schließen des Fensters aus. Zudem scheinen die zentralen Positionen zwischen lang- und kurzlebigen Parts nicht genau überein zu stimmen. Auch ist über den hier betrachteten zeitlichen Bereich kein signifikanter Anstieg beziehungsweise Abfall irgendeines Signals um $2.7 \mathrm{eV} \mathrm{zu}$ beobachten, was für eine weitaus höhere Lebensdauer des langlebigen Untergrunds verglichen zum gewählten Zeitfenster von $1 \mathrm{~ms}$ spricht. Im Gegensatz dazu zeigen die Signale um $2.0 \mathrm{eV}$ für die zwei Probensysteme zwar spektral sehr unterschiedliche aber im Zeitverhalten Rekombinationsraten in vergleichbarer Größenordnung, was durch einen signifikanten Anstieg als auch Abfall im gewählten Zeitfenster zu erkennen ist. Die Lebensdauer im undotierten AlN scheint dabei etwas langlebiger zu sein. Eine genaue Analyse dieser Bänder findet noch in Kapitel 5.3 und 5.4 statt.

Die abrupte Änderung der Intensitäten aufgrund der sehr unterschiedlichen Zeitskalen der Lumineszenz um $2.7 \mathrm{eV}$ bei der Verwendung des Chopperrads kann für eine bessere spektrale Separation genutzt werden. In Abbildung 5.10 wurden daher zwei unterschiedliche zeitliche Bereiche integriert und zu zwei zeitunabhängigen Spektren zusammengefasst. Bedingt durch die Messmethode mit der Streak-Kamera liegen dabei die zu erreichenden Zählraten typischerweise deutlich unter denen bei einer Nutzung des zeitunabhängigen Aufbaus mit dem $1 \mathrm{~m}$-Spektrometer. Jedoch ergibt sich hier die Möglichkeit einer weitgehenden Trennung des langlebigen Anteils vom kurzlebigen (siehe Abbildung 5.10(a)). Jener kann durch lediglich eine 


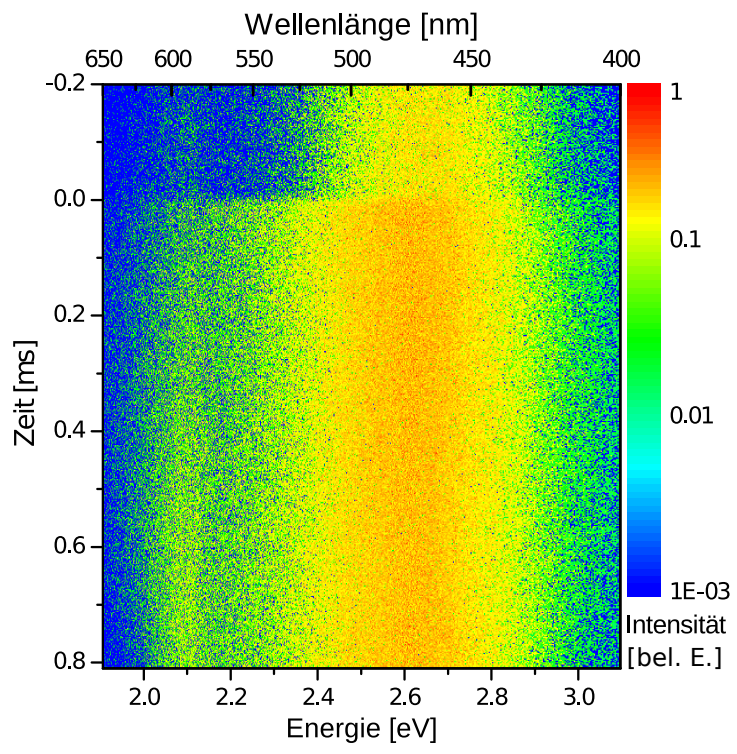

(a) AlN

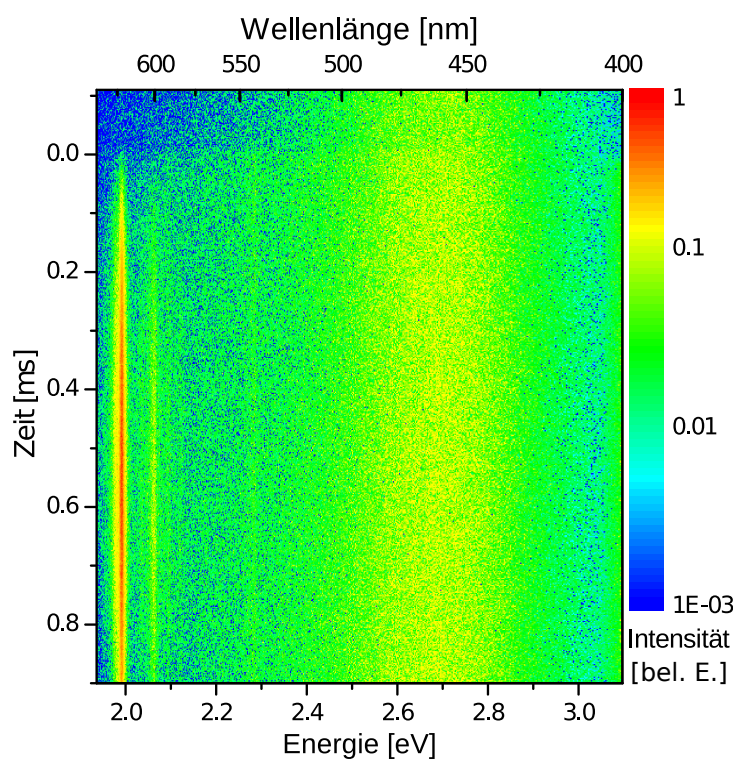

(c) $\mathrm{AlN}+\mathrm{Eu}$

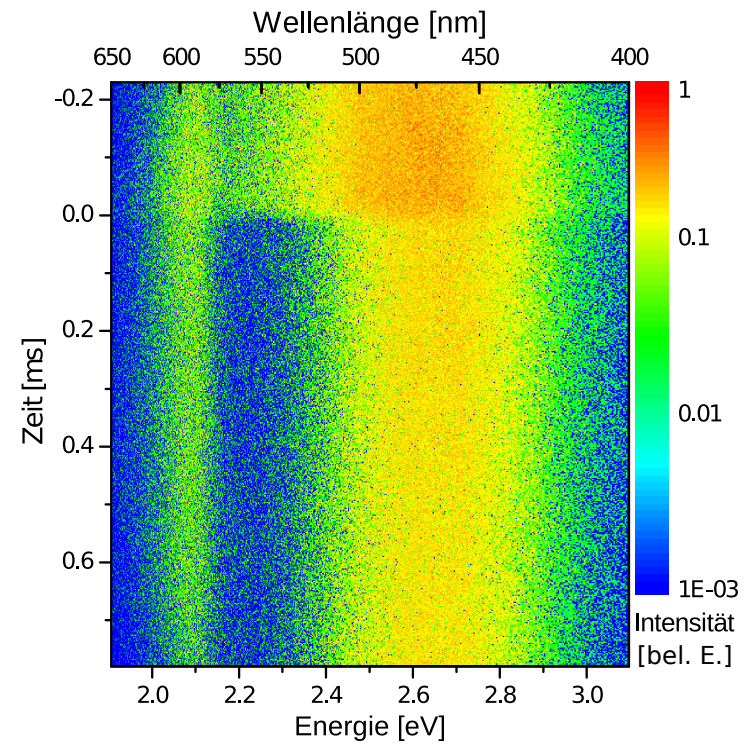

(b) AlN

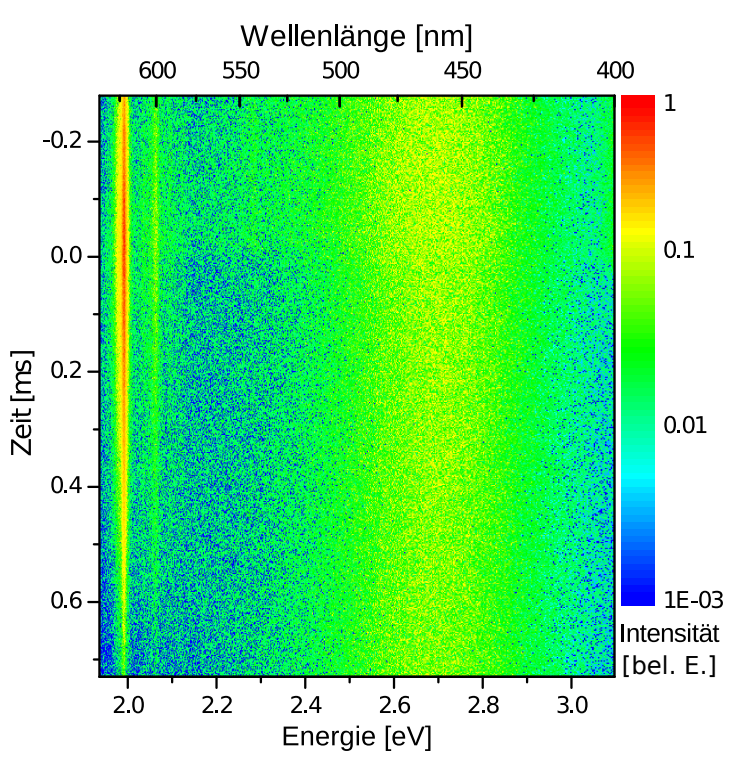

(d) $\mathrm{AlN}+\mathrm{Eu}$

Abbildung 5.9: Intensitätsverteilungen für Messungen mit dem Chopperrad für ein sich öffnendes (a, c) als auch ein sich schließendes (b, d) Fenster bei 290 K. Das beim sich öffnenden Fenster vorher noch vorhandene Signal stammt noch aus einem früheren Zyklus und ist aufgrund seiner langen Lebensdauer noch als Untergrund $\mathrm{zu}$ registrieren. 
Normalverteilung gut abgebildet werden und korrespondiert mit einer ermittelten Peak-Wellenlänge von 2.6556(6) eV sehr gut mit der Position des Hauptpeaks aus den zeitunabhängigen Spektren bei Raumtemperatur. Die Halbwertsbreite liegt mit $0.369(4) \mathrm{eV}$ etwas unterhalb derer aus den weiter oben präsentierten Messungen. Eine Anpassung des langlebigen als auch des kurzlebigen Anteils zusammen über eine einzelne Normalverteilung führt auch schon wie bei den vorher zeitunabhängigen Spektren nicht zu einer guten Repräsentation der Messdaten. Um eine Konsistenz der Daten untereinander zu wahren wurde deshalb eine Anpassung mit zwei Normalverteilungen in Betracht gezogen, wobei bei einer Verteilung der Mittelwert als auch die Halbwertsbreite auf die vorher bestimmten Werte fixiert wurden. Der daraus resultierende Fit ist in Abbildung 5.10 (b) dargestellt und führt zu einer im niedrigeren energetischen Bereich befindlichen Verteilung mit einem Maximalwert bei $2.488(9) \mathrm{eV}$. Dieser liegt damit etwas höher als die für diese Temperaturen bestimmten Werte aus den zeitunabhängigen Messungen.

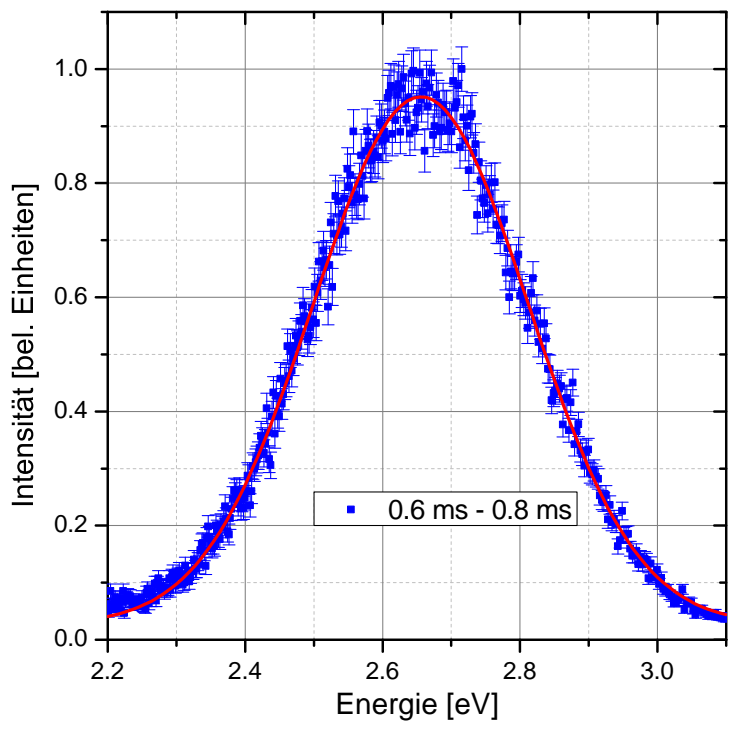

(a)

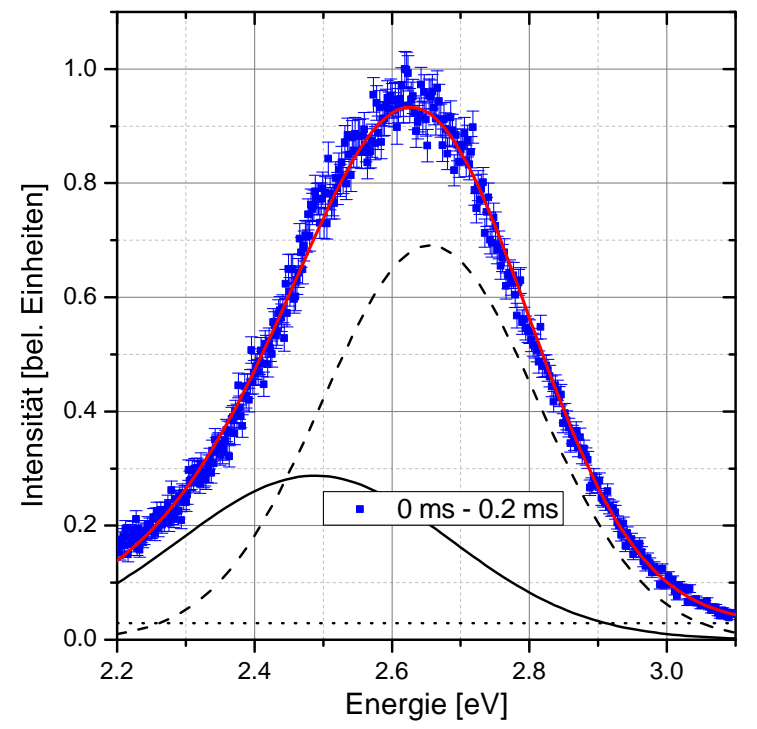

(b)

Abbildung 5.10: Integrierte Spektren in AlN aus einer Messung dargestellt in Abbildung 5.9 (b) für verschiedene Zeitbereiche. (a) Spektrum des langlebigen Anteils angepasst nach 5.5 zuzüglich konstantem Hintergrund. (b) Spektrum im Zeitbereich während der Anregung. Der Fit (rote Linie) erfolgte über zwei Normalverteilungen nach 5.5 und Hintergrund (gepunktet), wobei die Position als auch die Halbwertsbreite des langlebigen Anteils (gestrichelt) aus den Ergebnissen aus (a) übernommen wurden.

Um den bei einer Aufnahme von einer Millisekunde nahezu konstanten Signalverlauf des langlebigen Anteils um $2.7 \mathrm{eV}$ in seiner Lebensdauer spezifizieren zu 
können, wurden zunächst Messungen mit dem Trigger-Generator (siehe Kapitel 3.3.5) bei einer Wahl von 16 Zeitfenstern durchgeführt. Dabei wurden sowohl der ansteigende als auch der abfallende Anteil registriert. Die aus einer exponentiellen Anpassung resultierenden Lebensdauern sind in Abbildung 5.11(a) dargestellt. Eine eindeutige Signifikanz der Lebensdaueränderung mit der Temperatur ist dabei nicht zu erkennen. Jedoch fällt eine im Verhältnis zu den resultierenden Unsicherheiten deutlich zu große Streuung der Messdaten auf. Ursächlich dafür könnten noch andere in diesen Zeitbereichen überlagerte Rekombinationsprozesse sein. Da die bestimmten Lebensdauern im niedrigen zweistelligen Millisekundenbereich liegen, wurde die Messreihe mit nunmehr 56 Zeitfenstern und einer höheren Statistik wiederholt. In Abbildung 5.12 (a) ist eine Aufnahme des gleichen spektralen Bereichs wie in Abbildung 5.9 (a), jedoch über einen größeren Zeitraum, dargestellt. Der spektrale Unterschied zwischen geöffnetem und geschlossenem Fenster des Chopperrads ist dabei infolge der Dominanz des langlebigen Anteils wesentlich geringer ausgeprägt als noch bei den Aufnahmen mit kurzen Zeitbereichen. Korrespondierend dazu sind in Abbildung 5.12(b) der zeitliche Anstieg sowie Abfall gezeigt. Eine komplette Anpassung des Signals nach Gleichung A.10 auch unter Zuhilfenahme von bis zu drei Lebensdauern schlägt fehl. Daher wurde sich für eine rein exponentielle Anpassung des Verlaufs für zeitlich weit vom Verschlusszeitpunkt entfernte Ereignisse entschieden, um einen möglichst geringen Untergrund von Signalen mit kurzlebigeren Anteilen zu erhalten. Die aus diesen Fits bestimmten Lebensdauern sind in Abbildung 5.11(b) aufgetragen und können durch Gleichung 5.20 gut beschrieben werden. Im Gegensatz zu den vorherigen Aufnahmen dieser Lumineszenz zeigt sich eine wesentlich geringere Streuung der Messdaten, was auch gut zu den ermittelten Fehlern korrespondiert. Die aus der Anpassung berechnete Aktivierungsenergie für jene langlebige Lumineszenz beträgt $E_{a}=18(5) \mathrm{meV}$.

\section{Anregung mit anderen Wellenlängen}

Die bis hierher präsentierten Messungen in AlN wurden alle mit einer Anregungswellenlänge von $\lambda_{e x}=267 \mathrm{~nm}$ realisiert, da jene über eine relativ hohe Intensität als auch eine hohe Energie von $4.64 \mathrm{eV}$ verfügt. Um die Bandlücke von AlN zu überwinden, wären jedoch Anregungsenergien $>6 \mathrm{eV}$ nötig, was sich mit dem hier vorgestellten Lumineszenzaufbau nur mit der FHG $(3+1)$ realisieren lässt. Jene besitzt aber aufgrund des geringen Wirkungsgrads bei der Erzeugung auch nach einer optimalen Justage eine wesentlich niedrigere Intensiät (mindestens zwei Größenordnungen geringer), was wiederum zu einem deutlich abgeschwächten Lumineszenzverhalten der Proben führt. Des weiteren ist für die hier betrachteten Kristalle nicht mit einer Bandkantenlumineszenz infolge der hohen Defektlumineszenz nach Tang et al. [74] zu rechnen. Auch zeigten an den Proben zwischenzeitlich durchgeführte Kathodolumineszenzexperimente keinerlei Hinweise auf eine Bandkantenlumineszenz. Jedoch können verschiedene Anregungsenergien, welche kleiner als die Bandlücke sind, zu unter- 


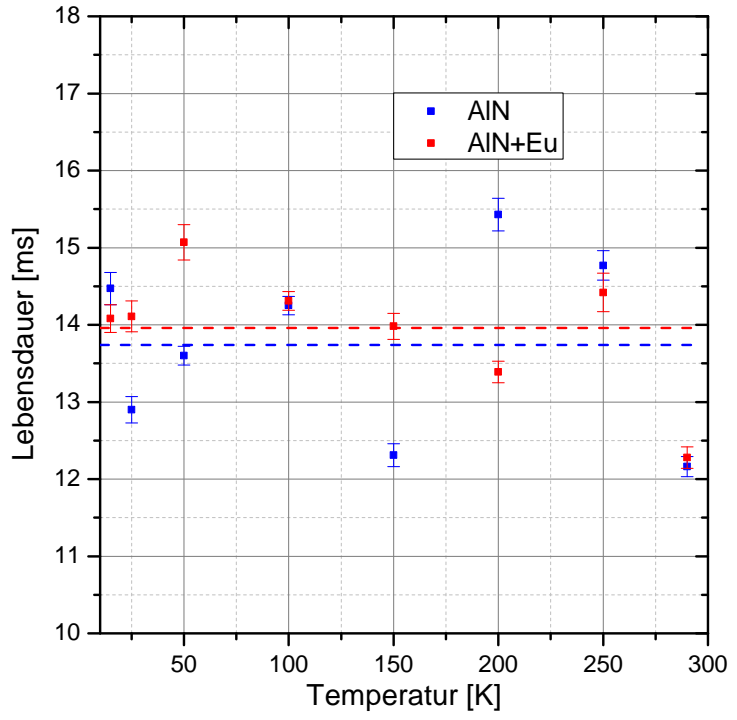

(a)

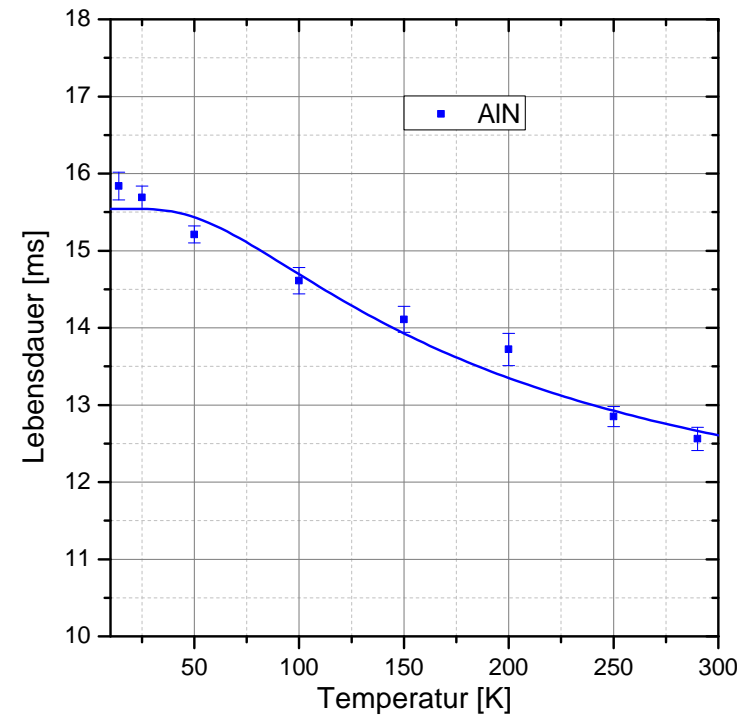

(b)

Abbildung 5.11: (a) Lebensdauer bestimmt aus exponentiellen Fits von Messungen mit Chopperrad und Trigger-Generator (16 Zeitfenster). Die eingezeichneten Linien spiegeln den gewichteten Mittelwert wieder. Die angegebenen Fehlerbalken stammen aus den Unsicherheiten der Anpassungen. (b) Lebensdauern aus exponentiellen Fits bei 56 Zeitfenstern mit zusätzlicher Beschreibung nach Gleichung 5.20. Auch hier stammen die Fehler aus den Unsicherheiten der bestimmten Parameter.

schiedlich ausgeprägten Lumineszenzbändern aufgrund der wellenlängenabhängigen Absorptionseigenschaften der Defekte führen.

Eine Anregung mit $\lambda_{e x}=377 \mathrm{~nm}(3.29 \mathrm{eV})$ führt zu einem starken Anstieg des kurzlebigen Anteils bei einer Wellenlänge um $2.5 \mathrm{eV}$. Jener lässt sich mit Hilfe von drei Lebensdauern nach Gleichung A.3 gut beschreiben (siehe Abbildung 5.13). Der vorher so dominante langlebige Untergrund ist dabei nicht mehr zu registrieren. Des weiteren fällt dieses kurzlebige Band durch seine hohe spektrale Breite von 0.565(1) eV über den fast kompletten sichtbaren Bereich auf.

Als eine weitere Anregungswellenlänge wurde die höherenergetische $\mathrm{FHG}(2+2)$ mit $\lambda_{e x}=220 \mathrm{~nm}(5.64 \mathrm{eV})$ gewählt. Während das Spektrum hier unterhalb von $3 \mathrm{eV}$ weitestgehend dem bei einer Anregung mit $267 \mathrm{~nm}$ entspricht, ist im Bereich um $3.9 \mathrm{eV}$ zusätzlich zum sehr kurzlebigen Signal ein langlebiger Anteil ebenda als auch bei $3.5 \mathrm{eV}$ hinzugekommen. Auf temperaturabhängige Messreihen wurde für diese zwei anderen Anregungsenergien verzichtet. 


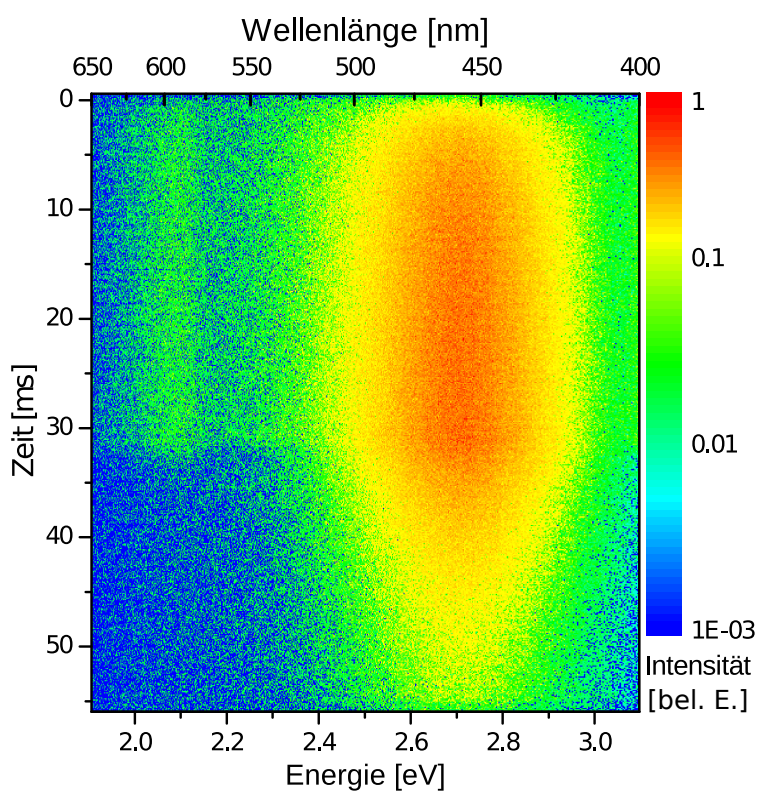

(a)

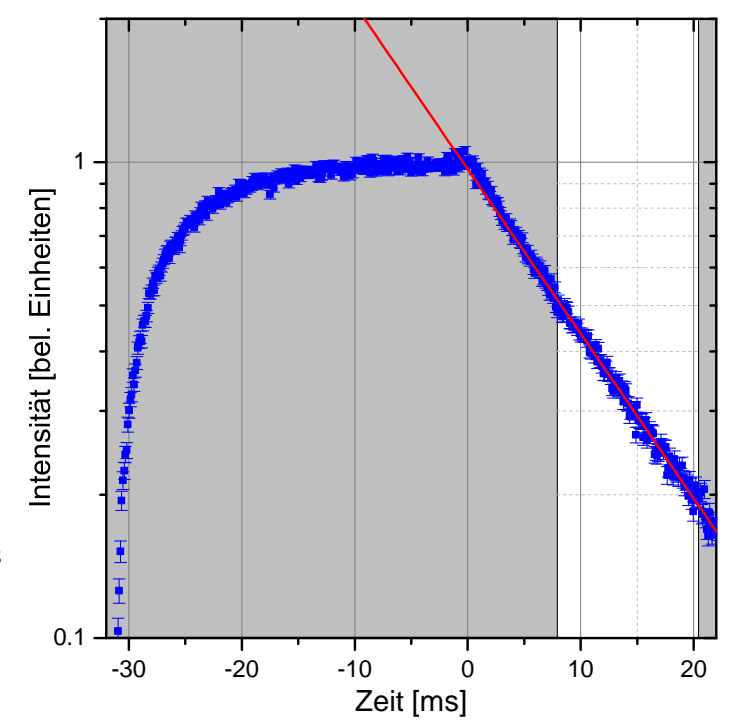

(b)

Abbildung 5.12: (a) Langlebige Komponenten einer Anregung mit Chopperrad und Trigger-Generator (56 Zeitfenster) in undotiertem AlN bei einer Temperatur von $290 \mathrm{~K}$. (b) Zeitliches Verhalten des Bereichs integriert von $2.63 \mathrm{eV}$ vis $2.88 \mathrm{eV}$. Angepasst wurde ein exponentieller Zerfall unter Berücksichtigung des nicht grau hinterlegten Gebiets.

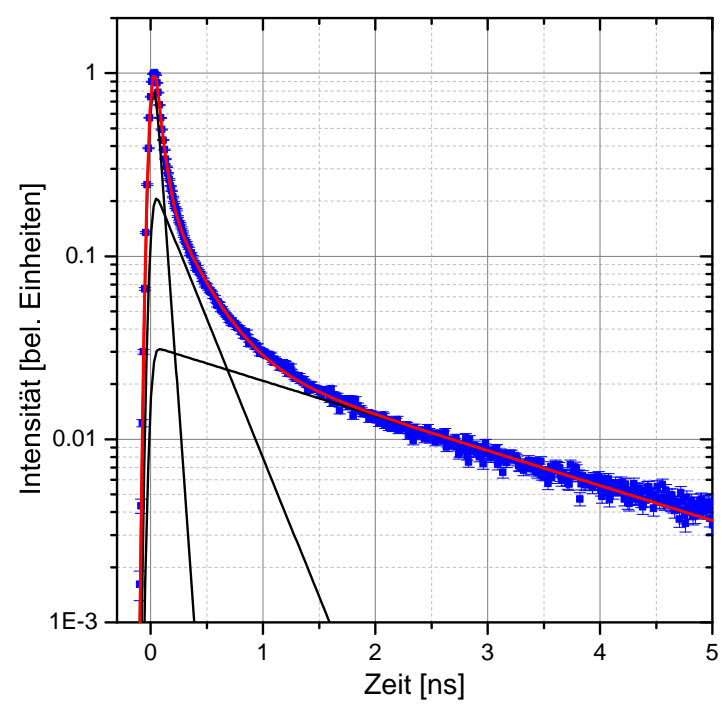

Abbildung 5.13: Zeitliches Verhalten des Lumineszenzsignals um $2.5 \mathrm{eV}$ (Integration aller Ereignisse von $2.07 \mathrm{eV}$ bis $2.76 \mathrm{eV}$ ) nach einer Anregung mit $\lambda_{e x}=$ $377 \mathrm{~nm}$. Bei der roten Kurve handelt es sich um eine Anpassung einer Summe von drei Lebensdauern (jeweils schwarz) nach Gleichung A.3 


\subsubsection{Diskussion und Zuordnung}

Unabhängig davon, ob das AlN mit Europium dotiert wurde oder undotiert vorliegt, zeigt sich für die durch Defekte erzeugte Lumineszenz in beiden Systemen ein ähnliches Verhalten. Über eine Beschreibung der zeitunabhängigen Spektren mit Hilfe von vier Normalverteilungen können die Messdaten gut reproduziert werden. Unter Zuhilfenahme der zeitaufgelösten Spektren wird deutlich, dass im betrachteten Materialsystem eine Vielzahl von Übergängen mit unterschiedlichsten Zeitkonstanten $\mathrm{zu}$ beobachten ist. Insbesondere um $2.7 \mathrm{eV}$ überlagern sich mindestens zwei breite Bänder, weshalb eine Beschreibung dieses Peaks mit lediglich einer Normalverteilung nicht möglich ist. Wie aus der Anregung mit $\lambda_{e x}=377 \mathrm{~nm}$ hervorgeht, existiert ein kurzlebiges sehr breites Defektband mit einer zentralen Wellenlänge um $2.5 \mathrm{eV}$. Dieses Band lässt sich mit drei Zeitkonstanten sehr gut beschreiben. Wahrscheinlich handelt es sich bei diesem Band nicht um einen einzelnen Übergang, sondern um mehrere überlagerte verschiedene Transferzentren, welche jedoch alle sehr kurze Lebensdauern aufweisen. In den Zeitunabhängigen Spektren repräsentieren sie dabei die in Abbildung 5.2 mit "1" und "3" markierten Bänder. Im gleichen energetischen Bereich existiert zudem ein sehr langlebiger Übergang, welcher insbesondere bei einer Anregung mit hohen Energien das Lumineszenzsspektrum dominiert. Unabhängig von diesen zentralen Signalen ist bei den hochenergetischen Anregungen und für alle Temperaturen auch ein extrem kurzlebiges Band um $3.9 \mathrm{eV}$ zu beobachten, welches im Fall sehr hoher Anregungsenergien noch durch einen langlebigen Transfer überlagert wird. Im Folgenden werden den einzelnen Bändern mögliche Ursachen auf Grundlage der in Kapitel 2.2 präsentierten Recherche der in der Literatur angegebenen Übergänge zugeschrieben.

\section{Signal bei $3.9 \mathrm{eV}$}

In diesem Energiebereich wurden zwei verschiedene Lumineszenzbänder beobachtet. Für den extrem kurzlebigen Transfer sind Band zu Defektübergänge als wahrscheinlichste Ursache zu benennen, da DAPs typischerweise um viele Größenordnungen längere Transferzeiten aufweisen. Als mögliche Kandidaten kommen hier ein Übergang vom Leitungsband auf einen Kohlenstoff-Defekt (Tabelle 2.5 ID 12) oder auch ein Transfer von Silizium in $D X$-Konfiguration zum Valenzband (Tabelle 2.5 ID 11) in Frage. Bei beiden möglichen Ursachen sind Fremdatome im Transfer mit involviert.

Für den langlebigen Übergang bei dieser Energie käme ein DAP-Transfer zwischen einem flachen Donator und einem Sauerstoff-Leerstellen-Komplex in Frage (Tabelle 2.5 ID 13, 14). Eine genaue Zuordnung um welchen Komplex beziehungsweise Ladungszustand es sich handelt, ist im Zuge der in Kapitel 2.2.2 schon dargestellten kontroversen Darstellung in der Literatur nicht ohne weiteres möglich. 


\section{Signal bei $2.7 \mathrm{eV}$}

Der das Defektspektrum dominierende Peak um $2.7 \mathrm{eV}$ weist eine sehr lange Lebensdauer im Bereich von 14 ms auf, was einen DAP-Übergang als wahrscheinlichsten Transfertyp favorisiert. In diesem Energiebereich liegen nur zugeordnete Übergänge dieser Art zwischen Stickstoffleerstellen und Kohlenstoff (Tabelle 2.5 ID 26) oder wie fast über den ganzen spektralen Bereich möglich, Transfers zwischen einem flachen Donator und einem Sauerstoff-Leerstellen-Komplex (Tabelle 2.5 ID 24, 32). Im Zusammenhang mit dem kurzlebigen Lumineszenzsignal um $3.9 \mathrm{eV}$, bei welchem ebenfalls Kohlenstoff als mögliche Ursache in Betracht gezogen werden kann, erscheint selbiger für die Lumineszenz um $2.7 \mathrm{eV}$ ebenfalls als plausibel. Da bei einer Anregung mit $\lambda_{e x}=377 \mathrm{~nm}$ dieses Signal nicht mehr zu detektieren ist, muss sich das dazugehörige Absorptionsband oberhalb von $3.3 \mathrm{eV}$ befinden. Auch dies spricht eher für Kohlenstoff als die Sauerstoff-Leerstellen-Komplexe, da sich ersterer für einen Transfer um $3.9 \mathrm{eV}$ auch entsprechend weit weg von der Kante des Leitungsbandes befinden muss, während bei den Komplexen zumeist eher flache Donatorzustände angenommen werden.

\section{Signal bei $2.5 \mathrm{eV}$}

Das Signal um 2.5 eV ist ein sehr kurzlebiges, sodass DAP-Übergänge als eher unwahrscheinlich angesehen werden können. Dabei scheint das sehr breite Defektband aus einer Kombination mehrerer einzelner Bänder mit unterschiedlichen Lebensdauern zu bestehen. Mit einer zentralen Wellenlänge um 2.5 eV befindet sich dieses Band am energetisch unteren Ende typischer Defektzustände in AlN. Von der Energie her passend sind Übergänge von Aluminiumleerstellen zum Valenzband (Tabelle 2.5 ID 29) oder Sauerstoff-Leerstellen-Komplexe mit einer Abregung ebenfalls in ein Valenzband (Tabelle 2.5 ID 33) als mögliche Ursache zu benennen. Bei den Komplexen müsste jedoch der Sauerstoffgehalt in den Proben aufgrund von drei Sauerstoffatomen pro Defekt schon sehr hoch sein. Eine andere Möglichkeit wäre gegebenenfalls noch ein Übergang vom Leitungsband in eine $D X$-Konfiguration des Sauerstoffs (Tabelle 2.5 ID 28), welche ebenfalls eine geringe Lebensdauer erwarten lässt. Jedoch gehen die meisten Theorien von einer Konfiguration des Sauerstoffs in Komplexen in Verbindung mit Leerstellen aus (siehe Kapitel 2.2.2), was eine Zuordnung dieses Signals nicht eindeutig gestaltet.

\subsection{Mangan-spezifische Lumineszenz}

Neben den beobachteten breitbandigen Defektlumineszenzen bei $2.7 \mathrm{eV}$ und $3.9 \mathrm{eV}$ zeigt das undotierte AlN auch eine Lumineszenz um $2.1 \mathrm{eV}$, die im Gegensatz zu den Erstgenannten bei allen gemessenen Temperaturen eine Unterstruktur aufweist (siehe Abbildung 5.14). Bei niedrigen Temperaturen sinkt die Breite der Peaks so 
weit ab, dass sich diese sogar voneinander separieren. Ein analoges Lumineszenzverhalten wurde schon von anderen Gruppen beobachtet und mit einer Lumineszenz in Verbindung mit Mangan in Zusammenhang gebracht (siehe Tabelle 2.5 ID 36 und 37). Insbesondere Karel et al. 212, 213, 250 untersuchten die spektralen Eigenschaften ihrer Proben für verschiedene Temperaturen (hauptsächlich oberhalb Raumtemperatur, nur zwei Messungen unterhalb), an welche in dieser Arbeit weiter angeknüpft wird.

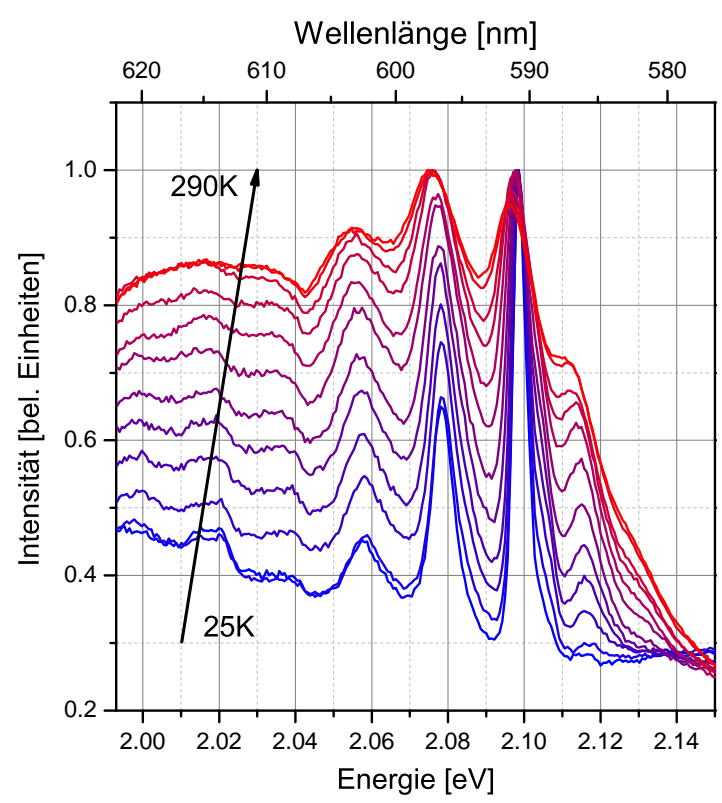

\begin{abstract}
Abbildung 5.14: Statisches Lumineszenzverhalten des undotieren AlN um $2.1 \mathrm{eV}$ für unterschiedliche Temperaturen $(25 \mathrm{~K}, 50 \mathrm{~K} \ldots 275 \mathrm{~K}, 290 \mathrm{~K})$ mit einer Anregung bei $\lambda_{e x}=267 \mathrm{~nm}$. Die Intensität ist für alle Messungen jeweils auf den höchsten Wert nach vorherigem Abzug des Untergrunds normiert. Die spektrale Auflösung bei diesen Messungen beträgt hier in etwa $2.8 \mathrm{meV}$ (Halbwertsbreite).
\end{abstract}

Zur besseren Vergleichbarkeit sind in allen Darstellungen die Intensitäten auf den Maximalwert normiert. Eine absolute Vergleichbarkeit jener für Messungen bei verschiedenen Temperaturen ist nicht ohne weiteres möglich, da durch den Schrägeinfall der Laseranregung und einer vom Aufbau her bedingten minimalen Verschiebung der Proben für jede Temperatur eine Nachjustage der Optiken durchgeführt werden musste, um eine maximale Lichtausbeute zu erreichen. Verglichen zu den anderen Defektbändern innerhalb des AlN fällt die integrierte Gesamtintensität der Lumineszenz um $2.1 \mathrm{eV}$ auf einen Wert unter $20 \%$ bei einer Abkühlung von $290 \mathrm{~K}$ auf $25 \mathrm{~K}$. Die Konzentration von schweren Elementen innerhalb des AlN liegt durchgeführten RBS-Messungen zufolge bei unter 0.1 at. \% (Nachweisgrenze in dieser Messung). Dies deckt sich auch mit Ergebnissen von Karel et al. [212], wonach bei höheren Konzentrationen die zu beobachtenden Linien bei tiefen Temperaturen deutlich breiter ausfallen müssten.

\title{
5.3.1 Beschreibung der Unterstruktur
}

Im Folgenden wird versucht, das temperaturabhängige Lumineszenzverhalten um $2.1 \mathrm{eV}$ genauer zu beschreiben. Nach Davydov haben bei einer starken Wechselwir- 
kung der Lumineszenzzentren mit der umgebenen Gitterschwingung die beobachteten Lumineszenzbänder in der Nähe der Resonanz die Form einer Normalverteilung und weiter entfernt die einer Lorentz-Funktion [317]. Der Unterschied zwischen diesen beiden Funktionen in der Nähe ihrer Maxima ist nicht sehr groß, sodass sich hier bei der Anpassung für reine Lorentz-Funktionen entschieden wurde. Bei der Beschreibung des Peaks ohne zusätzliche phononische Verschiebung ergibt sich damit folgende Form:

$$
I_{M 0}(E)=\frac{I_{0}}{2 \pi} \frac{\gamma_{0}}{\left(\frac{\gamma_{0}}{2}\right)^{2}+\left(E-E_{0}\right)^{2}} .
$$

Hierbei stehen $I_{0}$ für die integrierte Intensität, $\gamma_{0}$ für die Halbwertsbreite und $E_{0}$ für die energetische Position des Maximums. Im Fall der Stokes-/Anti-Stokesverschobenen Bänder gilt unter der Annahme einer simultanen Emission/Absorption von $n$ Phononen für die integrierte Intensität der dazugehörigen Bänder eine Poissonverteilung nach 318]:

$$
I_{n}=\frac{b^{n}}{n !} \exp \left(b^{n}\right)=\frac{b^{n}}{n !} I_{0} .
$$

Dabei stellt $b$ eine charakteristische Konstante für die mittlere Phononenzahl $\left(S_{ \pm}\right.$ in [153]) dar. Somit lassen sich die Intensitätsverteilungen für die Stokes- $\left(I_{S}\right)$ beziehungsweise Anti-Stokes $\left(I_{A}\right)$ verschobenen Resonanzen folgendermaßen darstellen:

$$
\begin{gathered}
I_{S}(E)=\frac{I_{0}}{2 \pi} \sum_{n=1}^{N} \frac{b_{S}^{n}}{n !} \frac{\gamma_{n}}{\left(\frac{\gamma_{n}}{2}\right)^{2}+\left(E-E_{0}+n \Delta E_{S}\right)^{2}} \\
I_{A}(E)=\frac{I_{0}}{2 \pi} \sum_{n=1}^{N^{\prime}} \frac{b_{A}^{n}}{n !} \frac{\gamma_{n}}{\left(\frac{\gamma_{n}}{2}\right)^{2}+\left(E-E_{0}-n \Delta E_{A}\right)^{2}} .
\end{gathered}
$$

Die Peaks sind hier jeweils um ganzzahlige Vielfache der Energie $\Delta E_{S}$ und $\Delta E_{A}$ vom Nullphononenübergang $(M 0)$ verschoben. Mit Blick auf die in Abbildung 5.14 dargestellten Resonanzen sind zur energetisch höheren Seite, auch schon bei niedrigen Temperaturen, Signalverbreiterungen zu erkennen. Diese treten sowohl beim Nullphononenübergang als auch bei den zu niedrigeren und höheren Energien verschobenen Übergängen auf. Für eine bessere Reproduktion des Signalverlaufes, aber um eine Überanpassung durch zu viele freie Parameter zu vermeiden, wird ein um die Energie $\Delta E_{R}$ verschobenes Replikat der vorher definierten Peaks in Betracht gezogen:

$$
I_{R}(E)=r \cdot\left[I_{M 0}\left(E-\Delta E_{R}\right)+I_{S}\left(E-\Delta E_{R}\right)+I_{A}\left(E-\Delta E_{R}\right)\right] .
$$


Als weiteren freien Parameter erhält jenes lediglich eine Skalierung seiner gesamten Intensität um den Faktor $r$. Somit ergibt sich als erstes Modell für die spektrale Anpassung des Lumineszenzsignals um $2.1 \mathrm{eV}$ eine Gesamtintensität:

$$
I_{T_{1}}(E)=I_{M 0}(E)+I_{S}(E)+I_{A}(E)+I_{R}(E)+c .
$$

Die Signale wurden vor dem Fit schon vom Untergrund bereinigt. Jedoch befinden sich unterhalb der betrachteten Peaks noch Ausläufer der breiteren höherenergetischen Defektlumineszenzen (siehe Kapitel 5.2.2 und 5.2.2), welche hier in nullter Näherung als konstantes Signal der Intensität $c$ modelliert werden. Eine Anpassung nach Gleichung 5.12 ist in Abbildung 5.15 für vier verschiedene Temperaturen dargestellt. Aufgrund der relativ freien Parameterwahl haben sich nur Fits mit maximal zwei Stokes- und zwei Anti-Stokes-Peaks als stabil erwiesen. Da mit steigender Temperatur die Peakbreite stark zunimmt, sind Prozesse höherer Phononenzahl nur noch schwer voneinander zu unterscheiden und zeigen bezüglich der Daten keine eindeutige Signifikanz mehr. Somit wurde auch der betrachtete Anpassungsbereich auf Energien oberhalb von $2.05 \mathrm{eV}$ beschränkt. Dies hat zur Folge, dass mit steigender Temperatur die Halbwertsbreite, insbesondere die des zweiten Stokes-verschobenen Peaks, unterschätzt wird (siehe Abbildung 5.15(c) und 5.15(d)). Bei einer niedrigeren energetischen Grenze findet allerdings eine starke Überschätzung jener statt, was wiederum aufgrund der gekoppelten Einzelintensitäten zu einer weit zu geringen Gesamthöhe führt.

Um eine Anpassung auch für Übergänge mit mehr als zwei Phononen durchzuführen, müssen die freien Parameter weiter eingeschränkt werden. Nach Pryce 318 können die Halbwertsbreiten für Prozesse mit $n$ Phononen folgendermaßen gekoppelt werden:

$$
\gamma_{n}=\sqrt{n} \cdot \gamma_{1}
$$

Somit verfügen die Multiphononenübergänge in Kombination mit den Einphononenübergängen über keine weiteren freien Parameter mehr. Ein Fit für die vorher schon gezeigten vier Temperaturen mit jeweils fünf Stokes-Peaks ist in Abbildung 5.16 dargestellt. Der Fitbereich wurde nach unten hin auf einen Wert von 2.045 eV erweitert, was verglichen zu der vorherigen Anpassung zu deutlich besseren Ergebnissen führt. Im Hinblick auf die Beschreibung bei höheren Temperaturen (siehe Abbildung $5.16(d))$ zeigt sich jedoch auch hier eine stärkere Diskrepanz bezüglich des eigentlichen Signalverlaufes. Zudem ist auch mit dieser Methodik eine Berücksichtigung des niederenergetischen Teils nicht möglich.

Da es sich bei diesem Anteil des Signals um eine relativ breitbandige Verteilung handelt, wird eine Anpassung jener durch eine Normalverteilung äquivalent zu Gleichung 5.5 in Betracht gezogen. Diese besitzt als freie Parameter ihre integrierte Gesamtintensität $I_{N 0}$, ihre zentrale Position $E_{N}$ und eine Standardabweichung $\sigma$. 


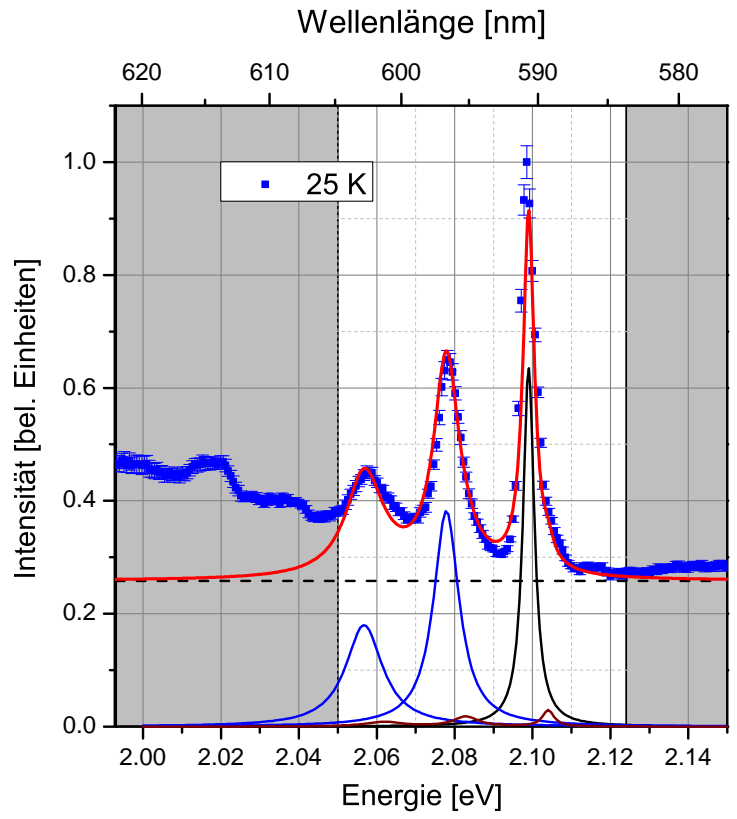

(a)

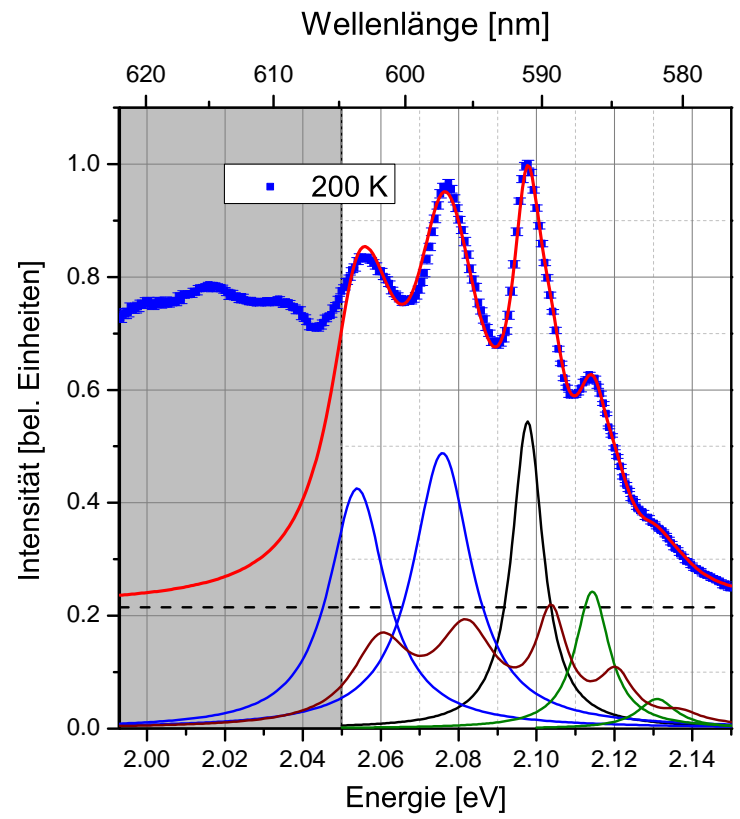

(c)

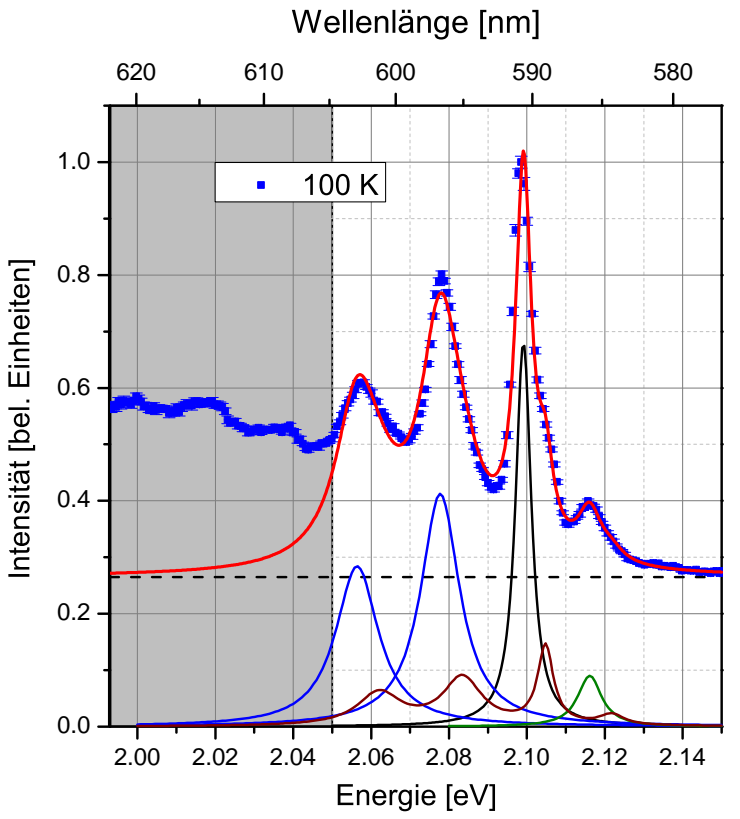

(b)

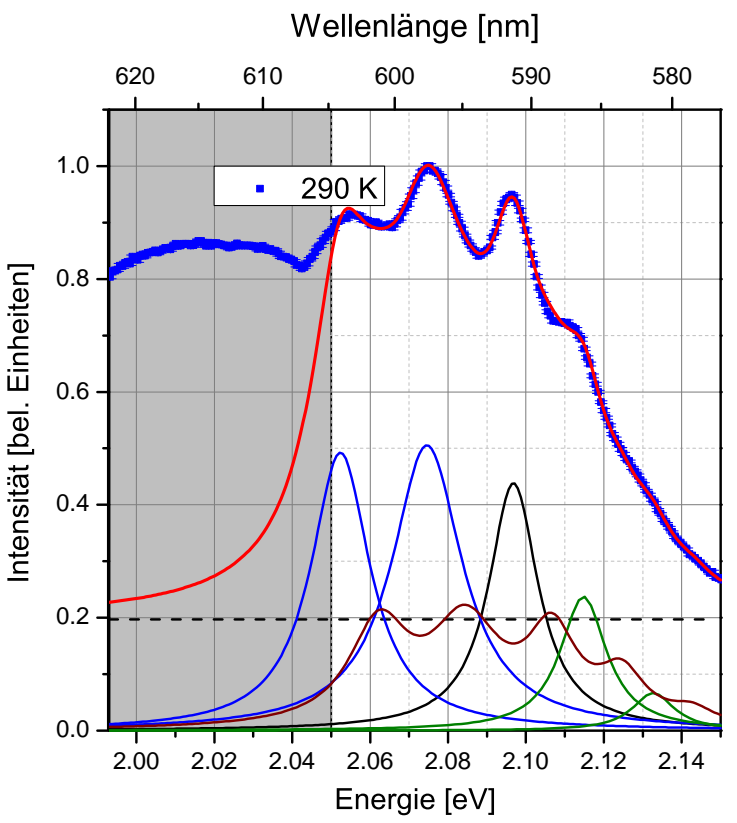

(d)

Abbildung 5.15: Anpassungen des Modells aus Gleichung 5.12 für vier verschiedene Temperaturen mit voneinander entkoppelten Halbwertsbreiten $\gamma_{n}$. Der jeweilig grau hinterlegte Bereich bleibt bei der Anpassung ohne Betrachtung. Pro Signal werden zwei Stokes-verschobene Resonanzen (blau) berücksichtigt. In der Messung bei $25 \mathrm{~K}$ (a) wird kein und bei $100 \mathrm{~K}$ (b) nur ein Anti-Stokes-Peak (grün) angepasst. Neben den dominanten Signalen wird zusätzlich ein dazu verschobenes, aber in seiner Form identisches Signal gefittet. 


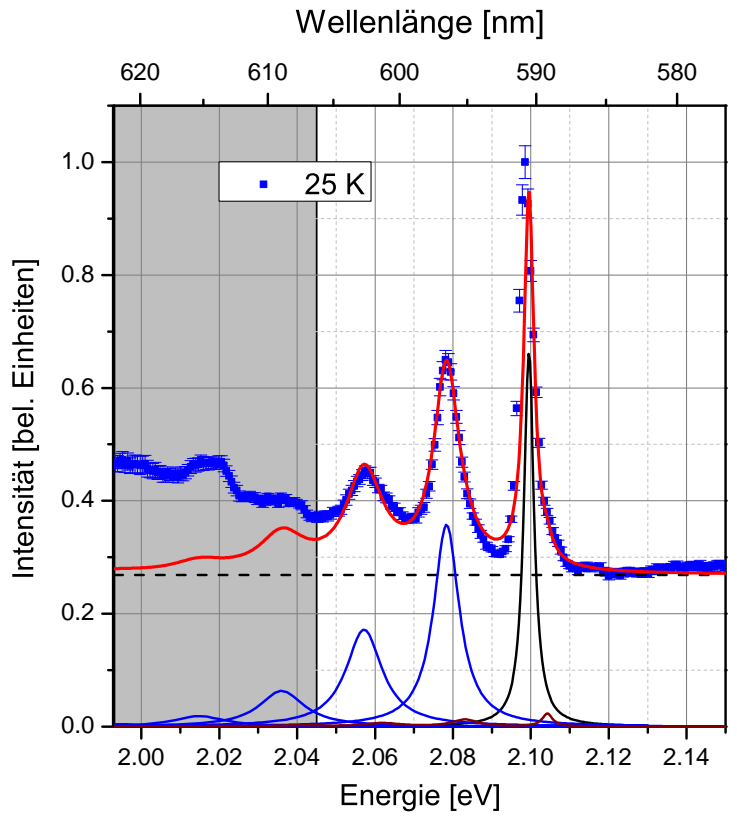

(a)

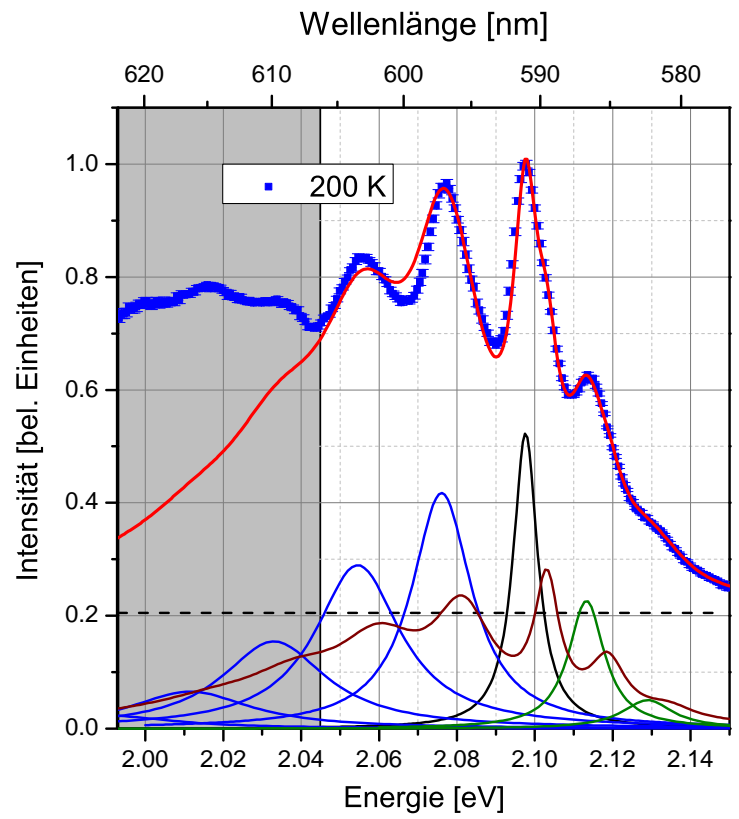

(c)

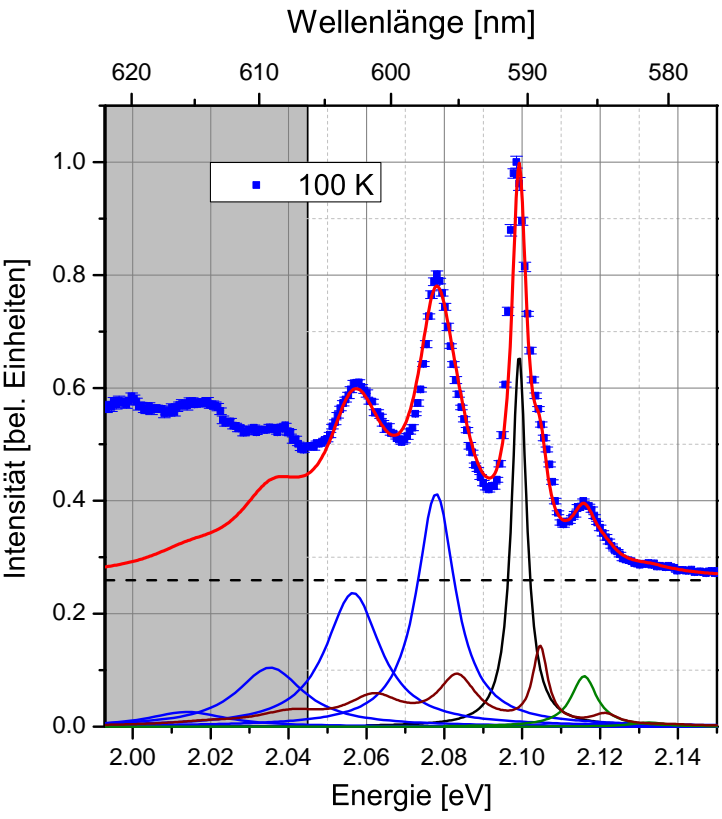

(b)

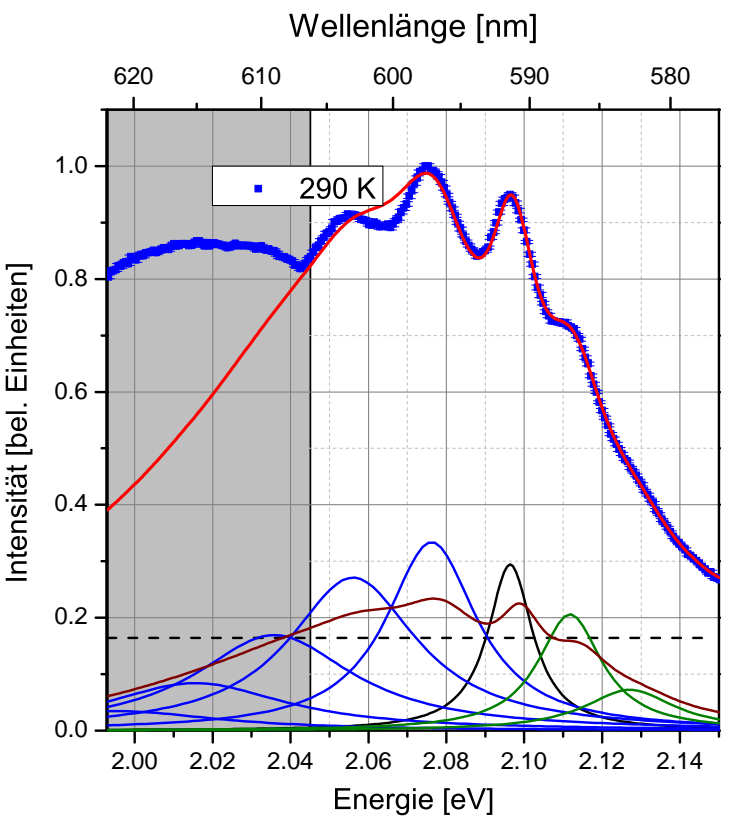

(d)

Abbildung 5.16: Anpassungen des Modells aus Gleichung 5.12 für vier verschiedene Temperaturen mit jeweilig gekoppelten Halbwertsbreiten $\gamma_{n}$ nach Gleichung 5.13 für Stokes und Anti-Stokes-Prozesse. Der grau hinterlegte Bereich bleibt bei der Anpassung unberücksichtigt. Es werden für jede Temperatur fünf Stokes- (blau) und für Temperaturen ab $75 \mathrm{~K}$ zwei Anti-Stokes-verschobene Peaks angepasst. Neben den dominanten Signalen wird zusätzlich ein dazu verschobenes, aber in seiner Form identisches Signal gefittet. 
Damit ergibt sich für die vollständige Beschreibung des Signals um $2.1 \mathrm{eV}$ folgendes Modell:

$$
I_{T_{2}}(E)=I_{M 0}(E)+I_{S}(E)+I_{A}(E)+I_{R}(E)+I_{N}(E)+c .
$$

Im Gegensatz zu den beiden vorherigen Modellen kann nun eine komplette Anpassung über den gesamten gemessenen Bereich erfolgen (siehe Abbildung 5.17). Insbesondere bei niedrigen Temperaturen ist somit auch eine Beschreibung des dritten Stokes-Peak möglich. Jedoch zeigt sich auch hier eine Unterschätzung des gemessenen Signals für einen bei etwa $2.015 \mathrm{eV}$ liegenden Peak, welcher somit durch das vorhandene Modell nicht weiter erklärt werden kann. Dennoch liefert der gesamte Fit eine deutlich bessere Beschreibung als die vorherigen Modelle. Bei allen Anpassungen wurden die Parameter für jede Temperatur getrennt bestimmt. Auch Parameter, welche über den gesamten Bereich hätten vielleicht konstant gehalten werden können, wie die Verschiebung der Replika $\Delta E_{R}$, wurden für jede Temperatur neu berechnet. Ein komplett gekoppelter Fit aller Temperaturen zugleich auch mit den Ergebnissen der Einzelanpassungen als Startbedingungen erwies sich aufgrund der Komplexität und der schieren Anzahl der Parameter für die Fitroutine (durchgeführt mit Gnuplot 4.6.4-2 [319]) nicht als stabil, wäre aber rein aus physikalischen Aspekten einer Einzelanpassung vorzuziehen.

Die energetischen Abstände $\Delta E_{S}$ und $\Delta E_{A}$ für die Stokes- beziehungsweise AntiStokes verschobenen Resonanzen sind in allen drei Ansätzen für jede Temperatur neu angepasst worden. Sie verhalten sich über den betrachteten Temperaturbereich nahezu konstant; mit einer leichten Tendenz zu niedrigeren Energien für hohe Temperaturen. Da die einzelnen Peaks durch diese äquidistante Separation gut beschrieben werden können, ist dies ein Hinweis auf eine Elektron-Phonon-Wechselwirkung. Die Ergebnisse aus den Anpassungen sind in der Tabelle 5.1 gegeben. Dabei zeigt sich, dass die energetisch nach oben verschobenen Linien einen in etwa $4 \mathrm{meV}$ geringeren Abstand zueinander haben, als die nach unten verschobenen, was für unterschiedliche Potentialformen im angeregten und im Grundzustand spricht [250]. Aufgrund der geringen Energie der Phononen verglichen zu den Gitterphononen in AlN [152] ist hier von pseudo-lokalen Phononen auszugehen. Im Vergleich zu Karel et al. [212, 250] zeigen sich sehr ähnliche energetische Abstände. Auch die absolute Position des Nullphononenübergangs liegt im Rahmen der gegebenen spektralen Auflösung nahezu exakt auf der dort angegebenen Position. Mit steigender Temperatur ist jedoch ein kontinuierlicher Drift hin zu niedrigeren Energien des Nullphononenübergangs zu beobachten (siehe Abbildung 5.18). Während sich bei den Modellen mit gekoppelten Parametern die zentrale Position sehr gleichmäßig zu niedrigeren Energien hin verschiebt, ist bei den Anpassungen mit komplett frei wählbaren Parametern bei Temperaturen oberhalb von $200 \mathrm{~K}$ eine starke Abflachung im Verlauf zu beobachten. Um den Kurvenverlauf theoretisch beschreiben zu können, wurden unterschiedliche Modelle an die Datensätze angefittet. Unter der Annahme von pseudo-lokalen Phononen konnte eine Anpassung mit einem Modell nach Skinner et al. [320] durchgeführt 


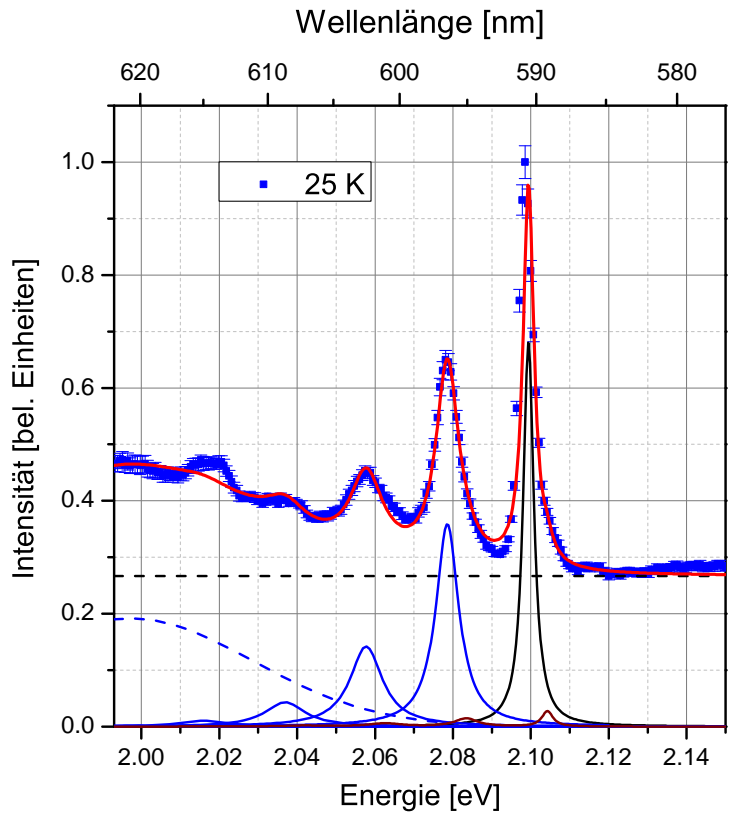

(a)

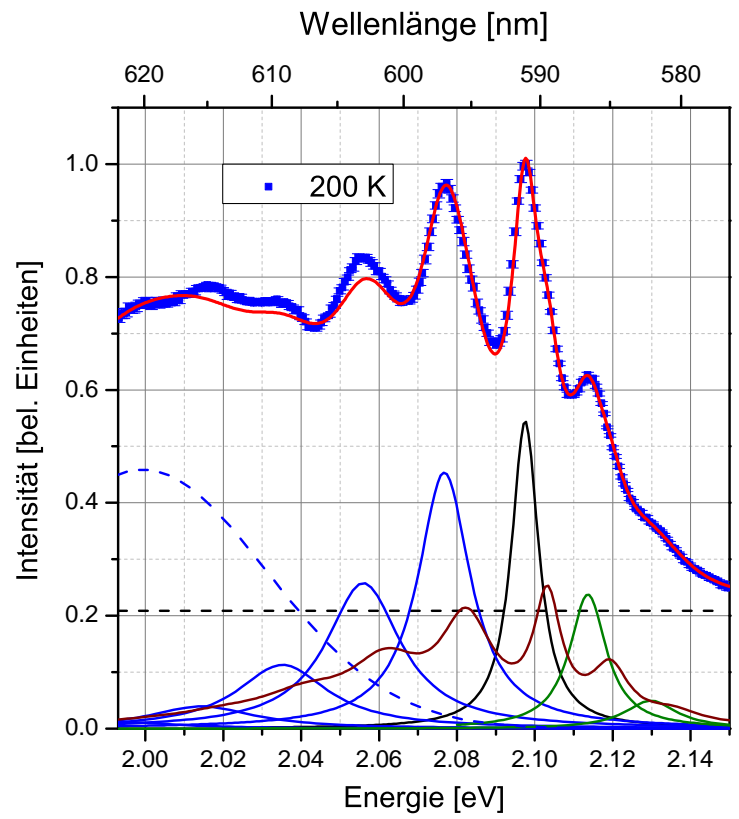

(c)

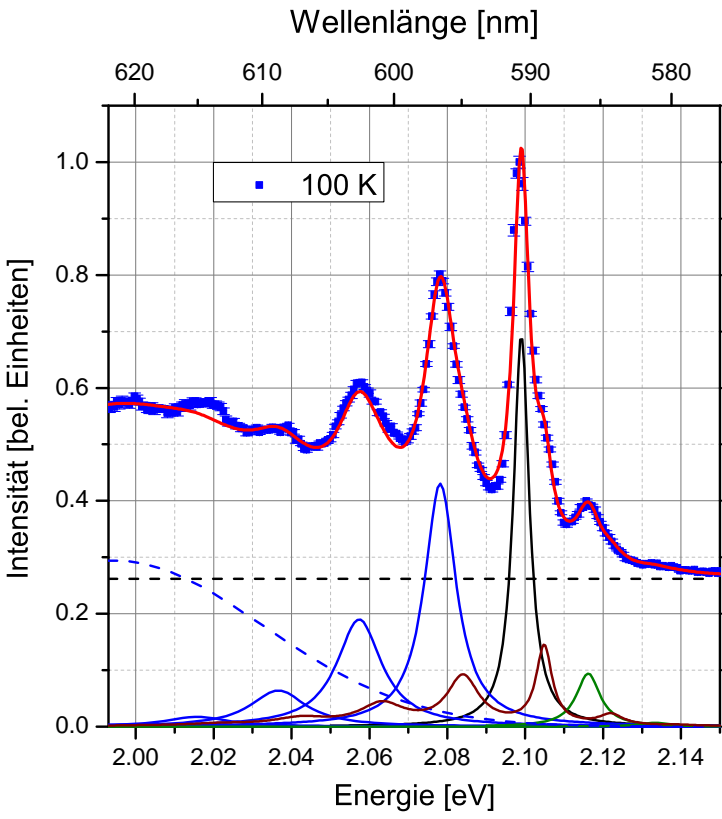

(b)

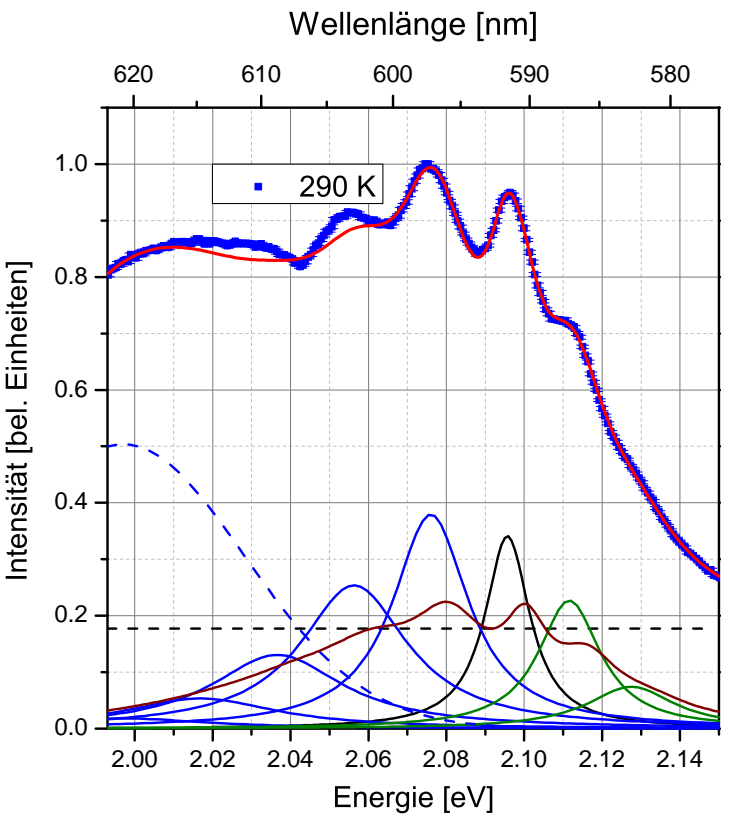

(d)

Abbildung 5.17: Anpassung des Modells aus Gleichung 5.14 für vier verschiedene Temperaturen mit jeweilig gekoppelten Halbwertsbreiten $\gamma_{n}$ nach Gleichung 5.13 für Stokes und Anti-Stokes-Prozesse. Im Gegensatz zu Abbildung 5.16 wird zusätzlich zur näherungsweisen Beschreibung des Signals im niedrigen energetischen Bereich eine Normalverteilung nach Gleichung 5.5 bei einer Energie um $2.0 \mathrm{eV}$ angepasst. Hierbei wird der komplette dargestellte Bereich vom Fit berücksichtigt. 
werden (Gleichung A.19), wobei es sich dabei um eine harmonische Näherung mit einer Taylor-Entwicklung der Potentialdifferenzen bis zur quadratischen Ordnung handelt. Angenommen wurde eine Verteilung der Phononenzustandsdichte nach der Form einer Lorentz-Funktion. Dieses Modell liefert für die pseudo-lokalen Phononen eine Energie von 35(5) meV und liegt somit etwas oberhalb der Energien bestimmt aus den Abständen zwischen den verschobenen Übergängen. Des weiteren zeigt sich bei Temperaturen oberhalb von $200 \mathrm{~K}$ eine deutliche Unterschätzung des energetischen Drifts. Alternativ kann auch eine Beschreibung in der Form nach Arrhenius:

$$
E_{0}(T)=a \cdot \exp \left(-\frac{E_{A}}{k_{\mathrm{B}} T}\right)+E_{0}(0)
$$

durchgeführt werden, wobei jene an sich für die Verschiebung nur für einen sehr eingeschränkten energetischen Bereich bei schwacher Kopplung gültig ist [320]. Ungeachtet dessen folgt diese Anpassung auch bei hohen Temperaturen den gemessenen Verschiebungen deutlich besser. Die Konstante $a$ ist hierbei ein Maß für die Kopplung. Mit einer ermittelten Aktivierungsenergie von 31.2(18) meV liegt der Wert etwas niedriger, als der des vorherigen Modells. Unter der Annahme, dass vielleicht auch akustische Phononen des Gitters als Ursache für das temperaturabhängige Verhalten in Betracht kommen können, wurde zudem eine Beschreibung nach dem Debye-Modell [320]:

$$
E_{0}(T)=a \cdot\left(\frac{T}{T_{D}}\right)^{4} \int_{0}^{T_{D} / T} \mathrm{~d} x \frac{x^{3}}{\exp (x)-1}+E_{0}(0)
$$

versucht. Auch hierdurch kann die Verschiebung des Maximums über den gesamten betrachteten Bereich gut abgebildet werden. Dabei wurde eine Debye-Temperatur von $T_{D}=330(40) \mathrm{K}$ bestimmt, welche jedoch weit unter der für wurtzitisches AlN (950 K [6]) liegt. Umgerechnet entspricht dies einer Energie von 28(4) meV, was noch etwas tiefer als die Ergebnisse der anderen beiden Modelle liegt. Jedoch ist die Annahme $T \ll T_{D}$ strenggenommen für den höheren Temperaturbereich nicht mehr erfüllt.

Im Hinblick auf die mit der Temperatur beobachtete Signalverbreiterung des Nullphononenübergangs liefern das Modell nach Debye und die allgemeine Herleitung für pseudo-lokale Phononen nach Skinner et al. [320] auch Voraussagen zur harmonischen Verbreiterung mit steigender Temperatur. Da bei den betrachteten Signalformen von Lorentz-Funktionen ausgegangen wurde, verhalten sich die minimale temperaturunabhängige Linienbreite $\gamma_{0}(0)$ (Breite beim Temperaturnullpunkt) und die harmonische Verbreiterung mit steigender Temperatur aufgrund der Invarianz gegenüber einer Faltung additiv. Für das allgemeine Modell für pseudo-lokale Phononen ergibt sich dabei die Form nach Gleichung A.20. Angepasst an die ermittelten Halbwertsbreiten zeigt sich für hohe Temperaturen ein Verlauf, welcher wie schon bei den Verschiebungen die eigentliche Änderung im Signal unterschätzt 
(siehe Abbildung 5.18(b) (gepunktet)). Insgesamt zeigt sich auch bei niedrigeren Temperaturen eine den bestimmten Halbwertsbreiten nur im Mittel folgende Kurve. Die daraus berechnete relativ hohe Energie der Phononen von 103(77) meV mit sehr großem Fehler spiegelt dies wieder. Ein Grund dafür liegt auch in der nötigen Bestimmung der Breite der Phononenverteilung im angewandten Fit.

Auch in der Herleitung nach Debye gibt es Voraussagen für die Verbreiterung durch akustische Gitterphononen nach der Form [320]:

$$
\gamma_{0}(T)=a \cdot\left(\frac{T}{T_{D}}\right)^{7} \int_{0}^{T_{D} / T} \mathrm{~d} x \frac{x^{6} \exp (x)}{[\exp (x)-1]^{2}}+\gamma_{0}(0) .
$$

Die Anpassung mit einer freien Wahl von $T_{D}$ verhält sich sehr instabil, weshalb in dem gezeigtem Fit in Abbildung 5.18(b) (durchgehend) die Temperatur auf den zuletzt ermittelten Wert von $T_{D}=330 \mathrm{~K}$ fixiert wurde. Verglichen zum vorherigen Modell zeigt sich eine bessere Abbildung der Daten mit einer leichten Überschätzung für tiefe Temperaturen. Jedoch sei auch hier darauf hingewiesen, dass die eigentliche Grundbedingung $T \ll T_{D}$ für die höheren Temperaturen nicht erfüllt ist.

Weitere aus den Messdaten zu extrahierende Parameter sind die für jede Temperatur neu bestimmten Faktoren $b_{S}$ und $b_{A}\left(S_{ \pm}\right.$in [153]). Unter der Annahme einer vernachlässigbaren Dispersion der beteiligten Moden haben jene für die Phononenemission beziehungsweise -absorption folgende vereinfachte Form [250]:

$$
\begin{gathered}
b_{S}=S \cdot\left[\frac{1}{\exp \left(\frac{E_{S}}{k_{\mathrm{B}} T}\right)-1}+1\right] \\
b_{A}=S \cdot\left[\frac{1}{\exp \left(\frac{E_{A}}{k_{\mathrm{B}} T}\right)-1}\right] .
\end{gathered}
$$

Hierbei wurde angenommen, dass der Faktor $S$ (temperaturunabhängiger HuangRhys-Faktor) sowohl für die Absorption als auch für die Emission die gleiche Größe besitzt. Ein simultaner Fit der beiden Gleichungen 5.18 und 5.19 ist in Abbildung 5.19 gegeben. Die Ergebnisse aus den Anpassungen für die drei unterschiedlichen Modelle sind in Tabelle 5.1 dargestellt. Im Fall freier Halbwertsbreiten werden die berechneten Energien verglichen zu den Abständen zwischen den Peaks stark überschätzt, während bei den Modellen mit gekoppelten Parametern die daraus gewonnen Werte nahe an den vorher bestimmten liegen, was die Annahme von phononisch verschobenen Peaks weiter unterstützt. Verglichen zur Literatur [250] zeigt auch der Parameter $S$ im Fall des dritten Modells die gleiche Größe.

\subsubsection{Lebensdauer}

Neben den statischen Messungen wurden auch zeitabhängige Experimente für die verschiedenen Temperaturen durchgeführt. Die Anregung wurde ebenso wie bei 


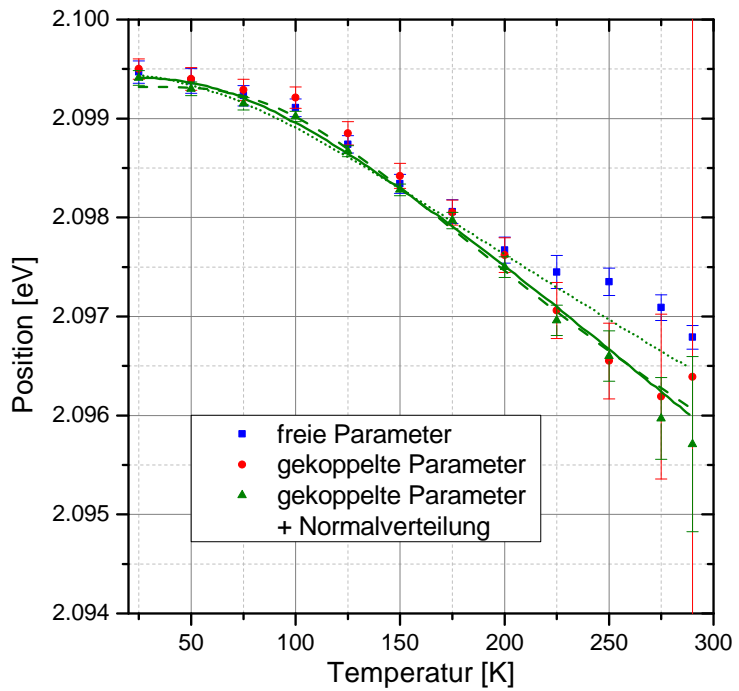

(a)

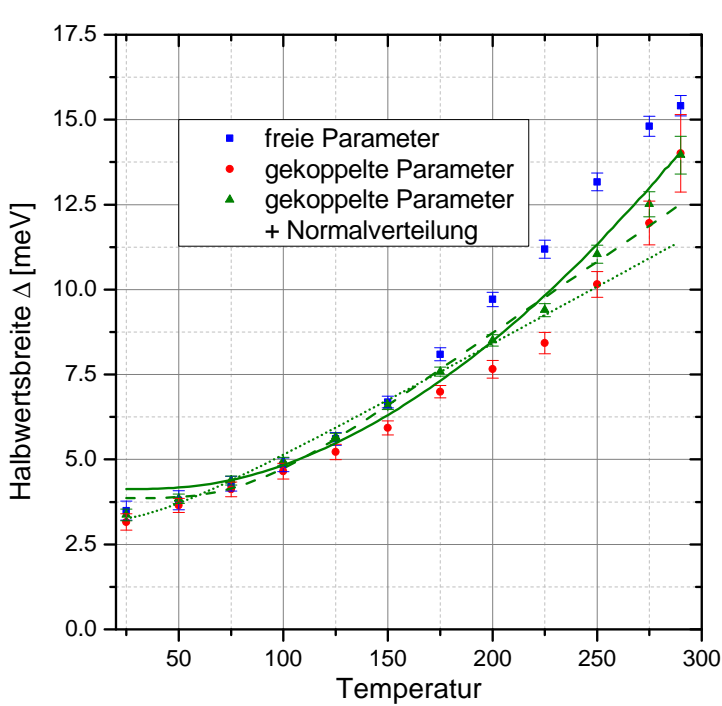

(b)

Abbildung 5.18: (a) Verschiebung des Maximums des phononlosen Übergangs (M0) mit der Temperatur. Für das Modell mit gekoppelten Parametern inklusive einer im Untergrund vorhandenen Normalverteilung wurden exemplarisch Anpassungen nach den Gleichungen 5.16 (durchgängig), 5.15 (gestrichelt) und A.19 (gepunktet) durchgeführt. (b) Halbwertsbreite des Nullphononenübergangs für die drei Modelle in Abhängigkeit von der Temperatur. Beim gleichen Modell wie vorher wurden hier die Gleichungen 5.17 (durchgängig), 5.21 (gestrichelt) und A.20 (gepunktet) angepasst.

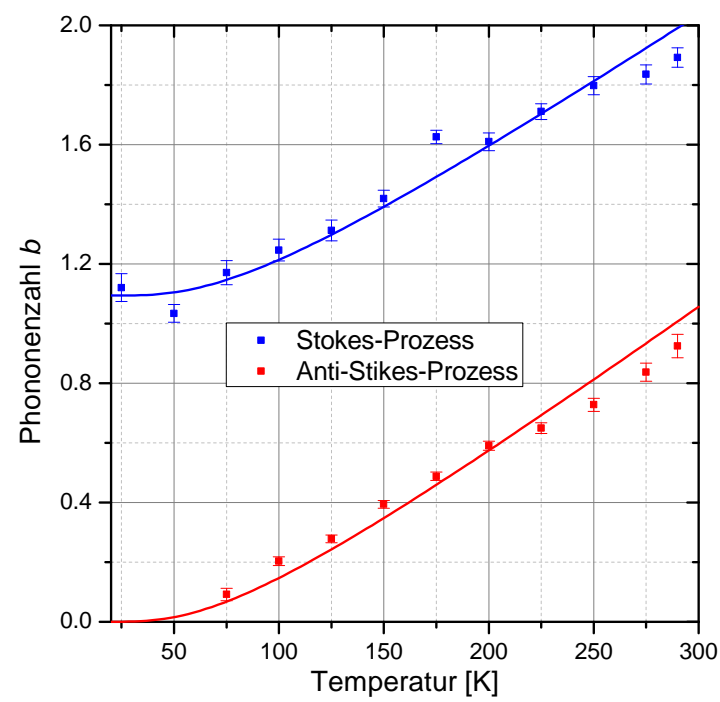

Abbildung 5.19: Charakteristische Konstante der mittleren Phonenzahl $b$ für Stokes- sowie Anti-Stokes verschobene Peaks für das Modell inklusive Normalverteilung. Angepasst wurden jeweilig die Gleichungen 5.18 und 5.19, wobei der Parameter $S=1.09(6)$ für beide Prozesse gekoppelt bestimmt wurde. 


\begin{tabular}{ccccccc}
\hline Modell & $\begin{array}{c}\Delta E_{S} \\
(\mathbf{m e V})\end{array}$ & $\begin{array}{c}\Delta E_{A} \\
(\mathbf{m e V})\end{array}$ & $\begin{array}{c}E_{S} \\
(\mathbf{m e V})\end{array}$ & $\begin{array}{c}E_{A} \\
(\mathbf{m e V})\end{array}$ & $S$ & $\begin{array}{c}\Delta E_{R} \\
(\mathbf{m e V})\end{array}$ \\
\hline frei & $21.5(5)$ & $17.7(6)$ & $63(21)$ & $31.1(16)$ & $1.57(8)$ & $6.3(16)$ \\
gekoppelt & $21.08(28)$ & $16.2(6)$ & $22.7(16)$ & $20.8(9)$ & $1.42(5)$ & $5.09(27)$ \\
gekoppelt+Gauß & $20.55(28)$ & $16.7(6)$ & $19.9(18)$ & $18.4(7)$ & $1.09(6)$ & $5.22(39)$ \\
\hline
\end{tabular}

Tabelle 5.1: Ergebnisse aus den Anpassungen für die drei verschiedenen Modelle. Die Stokes- $\left(\Delta E_{S}\right)$, Anti-Stokes-Verschiebung $\left(\Delta E_{A}\right)$ und die Verschiebung der Replika $\left(\Delta E_{R}\right)$ wurden aus den Gleichungen 5.9, 5.10 sowie 5.11 bestimmt und für die verschiedenen Temperaturen gemittelt. $E_{S}, E_{A}$ und a stammen aus den Gleichungen 5.18 und 5.19 .

den zeitunabhängigen Aufnahmen zur besseren Vergleichbarkeit auf $\lambda_{e x}=267 \mathrm{~nm}$ eingestellt. Da es sich bei den Lumineszenzen unter der Annahme von $\mathrm{Mn}^{4+}$ aber auch im Fall von $\mathrm{Mn}^{2+}$ um verbotene Übergänge innerhalb der Energieniveaus von einem Doublett- in einen Triplett- beziehungsweise Singulettzustand (Änderung der Multiplizität $\Delta s=1[213]$ ) handeln würde, wäre in diesem Fall mit einer verhältnismäßig langen Lebensdauer zu rechnen. Somit wurden die Messungen in Kombination mit dem Chopperad $(\sim 60 \mathrm{~Hz})$ und dem Trigger-Generator bei einer Aufnahme von 16 Zeitfenstern mit einer Länge von je $1 \mathrm{~ms}$ durchgeführt, wodurch sowohl die ansteigenden als auch die abfallenden Flanken der Signale registriert werden konnten (siehe Abbildung 5.20). Die Aufnahmen zwischen hohen und tiefen Temperaturen unterscheiden sich dabei signifikant voneinander. Während bei Raumtemperatur nur ein dominantes langlebiges Signal mit kontinuierlichem Anstieg als auch Abfall zu beobachten ist, zeigt sich bei Temperaturen unterhalb der $150 \mathrm{~K}$ ein immer weiter zunehmender Untergrund, hervorgerufen durch die Ausläufer einer im Verhältnis zum primären Signal kurzlebigen Defektlumineszenz (siehe Kapitel 5.2.2). Da jene aber auch bei höheren Energien direkt in der gleichen Aufnahme ohne das hier primär interessante Signal zu beobachten sind, kann deren zeitliches Verhalten für den entsprechenden Temperaturbereich getrennt bestimmt und in die Analyse mit einbezogen werden.

Im Fall von separaten Anpassungen des Anstiegs sowie des Abfalls bei $290 \mathrm{~K}$ nach Gleichung A.4 und A.7 zeigten sich sehr ähnliche Lebensdauern. Deshalb wurde sich bei den Fits zunächst für eine Beschreibung nach Gleichung A.10 entschieden. Auch ist bei den ermittelten Zerfallsraten der einzelnen Peaks kein im Vergleich zum Fehler her zu unterscheidender Wert gefunden worden, weshalb sich zum Erhalt einer besseren Statistik für einen Zusammenschluss der Werte von $2.07 \mathrm{eV}$ bis $2.11 \mathrm{eV}$ entschieden wurde. Dies stärkt die Theorie, dass es sich bei den beobachteten Übergängen um den gleichen physikalischen Ursprung handelt. Versuche unter 


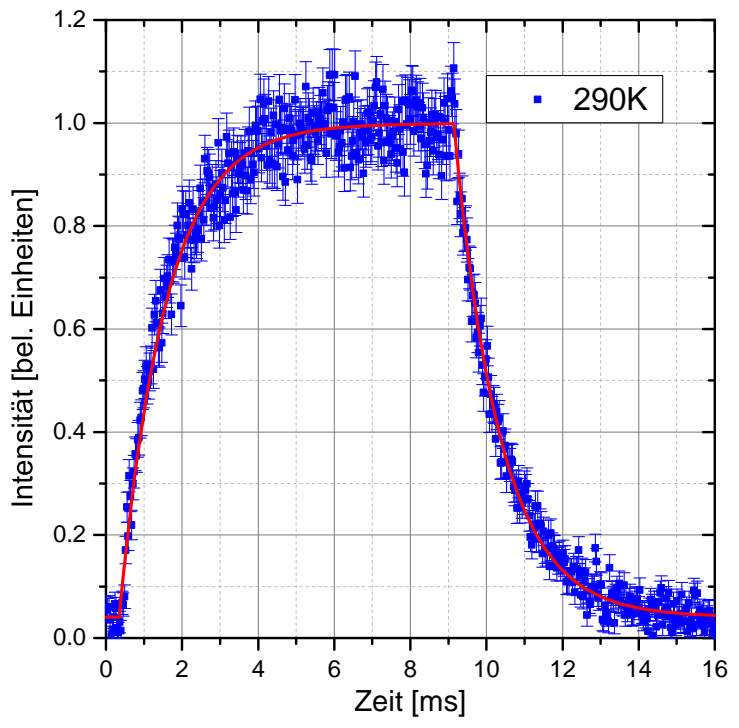

(a)

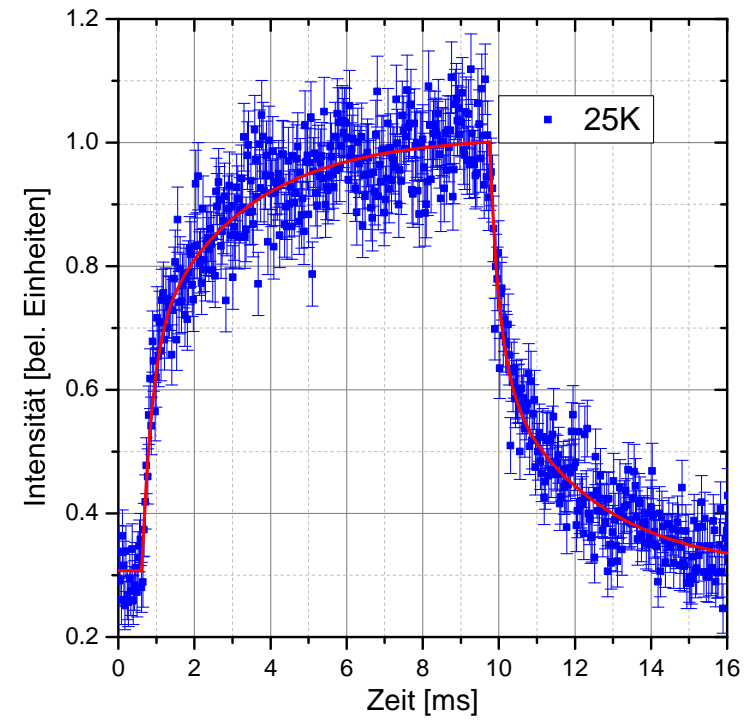

(b)

Abbildung 5.20: Zeitliches Verhalten des Lumineszenzsignals um $2.1 \mathrm{eV}$ mit einer Box-Anregung (siehe Abbildung $4.12(\mathrm{~g})$ ) für zwei unterschiedliche Temperaturen. Bei (a) fand eine Anpassung nach Gleichung A.10 mit konstantem Hintergrund statt, während bei (b) zwei überlagerte Lumineszenzen nach Gleichung A.10 mit konstantem Hintergrund angefittet wurden.

Berücksichtigung der zeitlichen Auflösung nach Gleichung A.12 ergaben mit einer Abweichung von unter 1\% die gleiche Lebensdauer. Um die Anzahl der freien Parameter möglichst gering zu halten, fand weiterhin Gleichung A.10 Anwendung. Für Temperaturen unterhalb von $150 \mathrm{~K}$ wird die Summe von zwei Gleichungen der Form A.10 angewandt, wobei zur Vermeidung einer Überparametrisierung für eine der beiden lediglich die Intensität als freier Parameter zur Verfügung steht. Die aus den Messungen ermittelten Lebensdauern sind in Abbildung 5.21(a) dargestellt. Dabei zeigt sich für Temperaturen unterhalb von $150 \mathrm{~K}$ eine nahezu konstante Lebensdauer, welche danach auf ein niedrigeres aber immer noch langlebiges Niveau abfällt. Jenes passt mit einer ermittelten Lebensdauer von 1.244(27) ms sehr gut zu den in der Literatur durch zeitaufgelöste Kathodolumineszenzmessungen beobachteten Zerfallskonstanten [212, 250]. Dort fanden die Autoren auch für höhere Temperaturen bis $400{ }^{\circ} \mathrm{C}$ keinerlei Temperaturabhängigkeit. Die zwei Plateaus der Lebensdauer mit einem Übergangsbereich bei $150 \mathrm{~K}$ können mit einem Einniveausystem nicht erklärt werden, da eine mögliche Löschung der Lumineszenz durch nichtradiative Prozesse nach einem startendem signifikanten Abfall zu einer immer weiter sinkenden Lebensdauer mit zunehmender Temperatur führen müsste. Das nächst komplexere System wäre eine Beschreibung über ein angeregtes Zwischenniveau (siehe Abbildung 
$4.11(\mathrm{~b}))$. Unter der Annahme eines im gemessenen Bereich temperaturunabhängigen strahlenden Übergangs wird jene Zerfallszeit auf den vorher ermittelten Wert von 1.244(27) ms festgelegt. Für eine Anpassung nach Gleichung A.11 sind die Lebensdauern für die Transferzeiten vom oberen zum Zwischenniveau in Abbildung 5.21(b) gegen die Temperatur aufgetragen. Der zu beobachtende Verlauf lässt sich über einen Mott-Seitz-Ansatz [316] der Form:

$$
\tau(T)=\frac{\tau_{0}}{1+C \cdot \exp \left(\frac{E_{a}}{k_{\mathrm{B}} T}\right)}
$$

gut reproduzieren. Der Parameter $\tau_{0}$ beschreibt dabei die Lebensdauer bei $0 \mathrm{~K}$. Für die Aktivierungsenergie wurde ein Wert von 160(20) meV bestimmt.

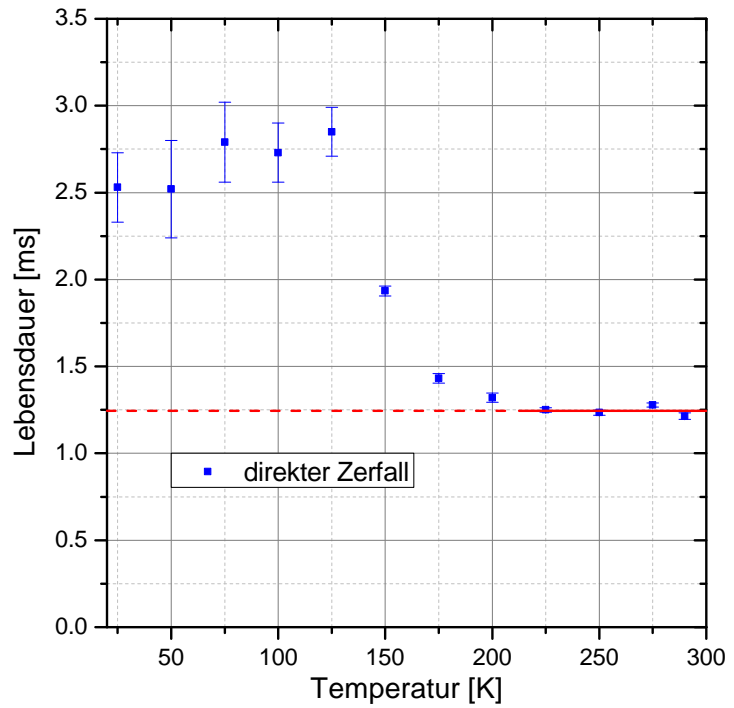

(a)

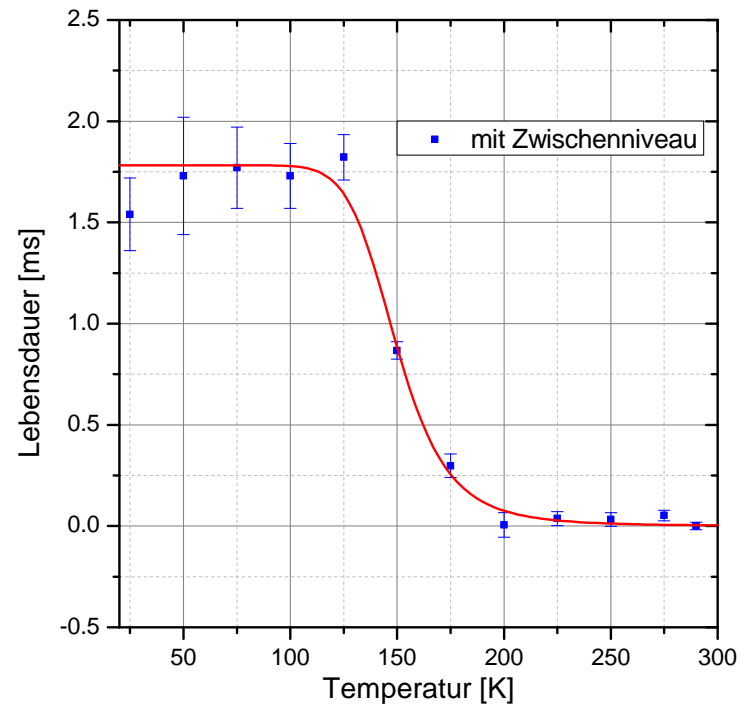

(b)

Abbildung 5.21: (a) Bestimmung der Lebensdauer der Lumineszenz um $2 \mathrm{eV}$ nach Gleichung A.10. Oberhalb von $200 \mathrm{~K}$ fällt sie auf einen Wert von 1.244(27) ms (rote Linie). (b) Lebensdauer unter der Annahme eines nicht strahlenden Zwischenniveaus nach Gleichung A.11. Die Lebensdauer des strahlenden Niveaus wird konstant mit 1.244(27) ms festgesetzt. Aus der Anpassung nach Gleichung 5.20 ergibt sich eine Aktivierungsenergie von $E_{a}=160(20) \mathrm{meV}$.

Aufgrund dieser Daten kann noch ein weiterer Versuch zur Beschreibung der Verbreiterung des Nullphononenübergangs mit der Temperatur unternommen werden. Nach einem Modell von Skinner 320] können auch Verbreiterungen über eine doppelte Arrhenius-Beziehung bei pseudo-lokalen Phononen beschrieben werden:

$$
\gamma_{0}(T)=a \cdot\left[\exp \left(\frac{-E_{A 1}}{k_{\mathrm{B} T}}\right)+\frac{1+E_{A 2} / E_{A 1}}{2} \exp \left(\frac{-E_{A 2}}{k_{\mathrm{B} T}}\right)\right]+\gamma_{0}(0) .
$$


Für die Energie $E_{A 2}$ wird versuchsweise der aus der Lebensdauer bestimmte Wert von $160 \mathrm{meV}$ eingesetzt. Damit ergibt sich für die erste Aktivierungsenergie eine Größe von $E_{A 1}=29.4(25) \mathrm{meV}$, was gut zu den in den Verschiebungen des Peaks mit der Temperatur gefundenen Parametern korrespondiert. Zudem zeigt sich verglichen zu den vorherigen Modellen eine bessere Reproduktion des Verlaufs der Daten (siehe Abbildung 5.18(b) (gestrichelt)).

\subsubsection{Diskussion}

Verglichen mit der Literatur ist sehr sicher davon auszugehen, dass es sich bei den vermessenen Lumineszenzen um $2.1 \mathrm{eV}$ in den hier untersuchten Proben um Übergänge des Mangan handelt. Die beobachteten Nebenpeaks verhalten sich mit der Temperatur wie für Übergänge mit zusätzlichen phononischen Absorptionen beziehungsweise Emissionen erwartet. Die Verschiebung insbesondere aber auch die Verbreiterung des Nullphononenübergangs lässt sich jedoch nicht zweifelsfrei allein mit den Modellen für pseudo-lokale Phononen erklären. Mangan substituiert im Falle eines tetravalenten Ladungszustandes höchstwahrscheinlich den Aluminiumplatz [213]. Dabei besitzt es in etwa die doppelte Masse wie Aluminium. Möglicherweise wird die beobachtete Verschiebung als auch die Verbreiterung durch eine Mischung aus den pseudo-lokalen sowie den umgebenen Gitterphononen hervorgerufen und lässt sich daher weder mit dem einen, noch dem anderen Modell allein komplett beschreiben. Die Änderung der Lebensdauer kann mithilfe eines Zwischenniveaus für den betrachteten Temperaturbereich gut reproduziert werden. Da der strahlende Übergang im Einklang mit der Literatur als temperaturunabhängig angenommen wurde, resultiert die Änderung der Lebensdauer nur aus dem Transfer eines zunächst nicht weiter zu spezifizierenden Niveaus. Dieser Transfer könnte zum einen von einem anderen langlebigen Defekt innerhalb des AlN stammen, wonach jener jedoch aufgrund der deutlich reduzierten Intensität bei niedrigen Temperaturen ebenfalls weniger stark angeregt werden müsste. Zudem dürfte dieser Defekt keine weiteren Zerfallskanäle mit niedrigeren Aktivierungsenergien als auch Lebensdauern im Bereich bis $160 \mathrm{meV}$ aufweisen. Zum anderen gäbe es die Möglichkeit, dass der Defektkomplex Mangan selbst über ein höher energetisches Niveau verfügt, welches in seinem Übergang zum niedrigeren strahlenden Zustand ebenfalls verboten oder in der Kopplung stark unterdrückt ist. Mit steigender Temperatur könnte dieser Übergang dann durch mehrere Phononen überbrückt werden.

\subsection{Europium-spezifische Lumineszenz}

Während in den undotierten Proben bei $2.0 \mathrm{eV}$ die Lumineszenz des Mangan eindeutig als verantwortlicher Ursprung identifiziert werden kann, ist dies bei den europiumdotierten Proben der Ladungszustand " $3+$ " jenes seltenen Erds. Die verhältnismäßig 
schmalbandigen Linien sind schon in den aufgenommenen Übersichtsspektren (siehe beispielsweise 5.1(b)) deutlich zu erkennen. Auf eine detaillierte temperaturabhängige Untersuchung aller Linien wurde aufgrund des erheblichen Mess- und Auswertungsaufwandes und dem hier in dieser Arbeit nicht primären Fokus auf die einzelnen Bänder verzichtet. Zudem existiert für die hier vorgestellten Proben schon eine Veröffentlichung, welche auf die energetischen Positionen der einzelnen Linien und deren Zuordnung zu den verschiedenen Energieniveaus zumindest für eine Temperatur eingeht (siehe dazu Referenz [228]). Dabei konnten aus theoretischen Betrachtungen ein Großteil der beobachtbaren Linien schon zugeordnet werden. Im Folgenden wurde sich hinsichtlich ihrer Prominenz im Spektrum auf die intensitätsstärksten Peaks beschränkt. Insbesondere der Bereich um $1.987 \mathrm{eV}$ wurde auch spektral genauer untersucht.

\subsubsection{Statisches Lumineszenzverhalten um $1.987 \mathrm{eV}$}

Zunächst wird auf die statische Lumineszenz um $1.987 \mathrm{eV}$ eingegangen. Hierfür wurden wie schon beim Mangan (siehe Kapitel 5.3) für jede untersuchte Temperatur jeweils 20 Messungen durchgeführt, gemittelt und die Fehler der berechneten Mittelwerte bestimmt. Die daraus resultierenden Spektren sind in Abbildung 5.22 zusammengefasst. Dabei sind drei sich teilweise gegenseitig überlappende schmale Lumineszenzbänder zu beobachten. Nach Gruber et al. 228 handelt es sich bei den zwei niederenergetischen Peaks um einen Transfer der Energielevel ${ }^{5} D_{0} \rightarrow{ }^{7} F_{2}$, welche dort die spektroskopische Bezeichnung " $A_{1} \rightarrow X_{1}$ " (mittlerer Peak) und " $A_{1} \rightarrow X_{2}$ " (niederenergetischster Peak) tragen. Eine Zuordnung des energetisch am höchsten liegenden Peaks fand indes nicht statt. Dieser wird daher im Folgenden als "? $\rightarrow$ ?" bezeichnet.

Bei den hier durchgeführten Messungen wurde darauf geachtet, dass die spektrale Auflösung durch ein entsprechend schmales Einstellen der Eingangs- und Ausgangsfilter möglichst weit unterhalb der beobachteten Linienbreiten liegt. Für den schmalsten Übergang bei niedrigen Temperaturen wird aber schon ein leichter Einfluss auf die ermittelten Halbwertsbreiten angenommen, da jene nur etwa um einen Faktor von 2.2 über der erreichbaren Auflösung liegen. Wie auch schon bei den Signalen des Mangan wird eine Beschreibung der Europium-Peaks über Lorentz-Funktionen der Form nach Gleichung 5.7 angestrebt. Dabei werden für jede Temperatur die jeweiligen Intensitäten $I_{0}$, Halbwertsbreiten $\gamma_{0}$ als auch die zentralen Positionen $E_{0}$ neu bestimmt. Zusätzlich wird ein konstanter Hintergrund, welcher durch Dunkelrauschen und Ausläufern von breitbandigen Lumineszenzen herrührt in den Anpassungen mit einbezogen. In Abbildung 5.23 sind exemplarisch die Ergebnisse aus den Fits für vier Temperaturen grafisch dargestellt. Hierbei ist zu beobachten, dass die jeweiligen Peaks auch weit abseits von den eigentlichen Maxima gut durch die gewählte Anpassung beschrieben werden können. Lediglich 


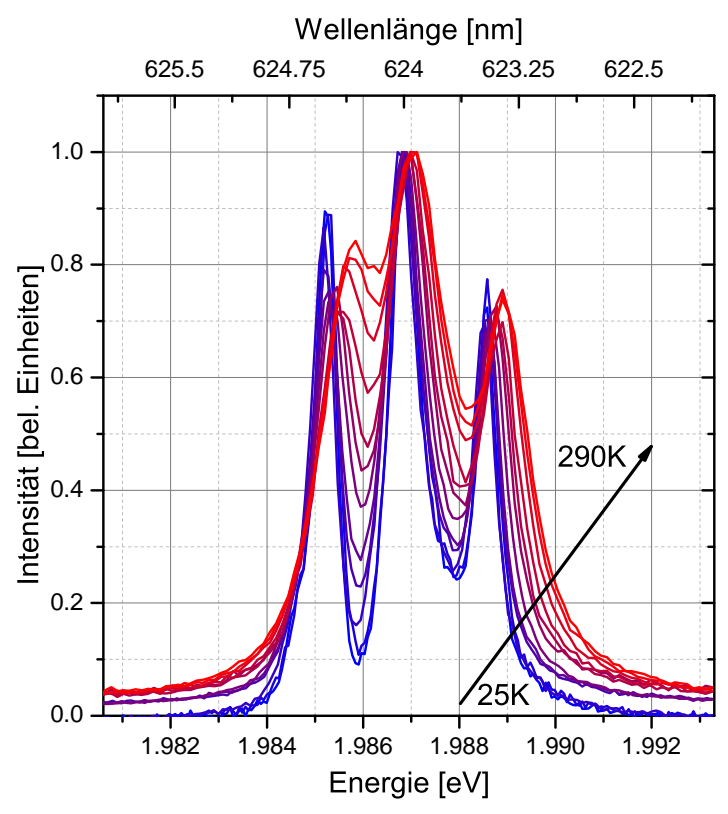

Abbildung 5.22: Statisches temperaturabhängiges Verhalten $(25 \mathrm{~K}$, $50 \mathrm{~K} . .275 \mathrm{~K}, \quad 290 \mathrm{~K}$ ) der intensiven Lumineszenz im europiumdotierten AlN um $1.987 \mathrm{eV}$ nach einer Anregung mit $\lambda_{e x}=267 \mathrm{~nm}$. Die Signale sind jeweils auf ihren Maximalwert normiert und beinhalten zum Teil noch vorhandene Untergundanteile. Die spektrale Auflösung in dieser Messung liegt bei $1.987 \mathrm{eV}$ bei unter $0.2 \mathrm{meV}$.

bei niedrigen Temperaturen zeigt sich zwischen den beiden niederenergetischen Signalen eine größere Diskrepanz zwischen Modell und Daten.

Die aus den Anpassungen ermittelten Änderungen der zentralen Positionen der Peaks sowie deren jeweilige Halbwertsbreiten sind in Abbildung 5.24 dargestellt. Im Gegensatz zu den schmalbandigen Peaks der Lumineszenzen des Mangan wandern die zentralen Maxima des Europium mit steigender Temperatur zu höheren Energien. Auch hier wurde eine Anpassung der Kurvenverläufe der Positionen als auch der Halbwertsbreiten nach einer Theorie von Skinner et al. [320 für pseudolokale Phononen angewandt. Insbesondere bei den Halbwertsbreiten kann dabei der Verlauf der Daten gut reproduziert werden. Für die ermittelten Positionen hingegen ergeben sich leicht größere Abweichungen. Vor allem bei den niedrigsten Temperaturen ist in den Daten ein leichter Sprung hin zu einer höheren Energie zu erkennen.

Im Gegensatz zu den phononischen Replika bei den Signalen des Mangan ist im hier betrachteten Temperaturbereich für die verschiedenen Intensitäten der drei Peaks kein spezifischer Trend zu erkennen (siehe Abbildung 5.25). Die beiden Lumineszenzen bewegen sich in etwa bei $50 \%$ der Intensität des stärksten Signals in der Mitte. Der zum selben Niveau zugehörige Peak ist dabei in seiner Intensität gegenüber dem unbestimmten Signal tendenziell leicht erhöht. Beide zeigen eine zueinander korrelierte Variation der relativen Intensität.

\subsubsection{Zeitabhängiges Lumineszenzverhalten}

Neben den spektralen Eigenschaften wurde auch das zeitabhängige Lumineszenzverhalten des Übergangs ${ }^{5} D_{0} \rightarrow{ }^{7} F_{2}$, sowie der benachbarten Übergänge ${ }^{5} D_{0} \rightarrow{ }^{7} F_{1}$ und ${ }^{5} D_{0} \rightarrow{ }^{7} F_{3}$ für einen Temperaturbereich bis $290 \mathrm{~K}$ untersucht. Da es sich bei 


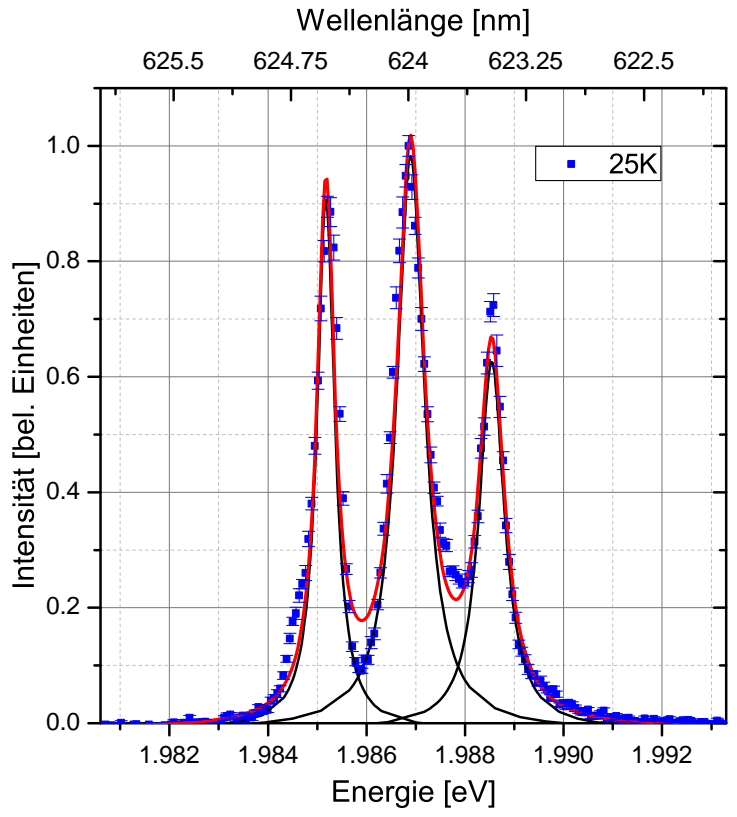

(a)

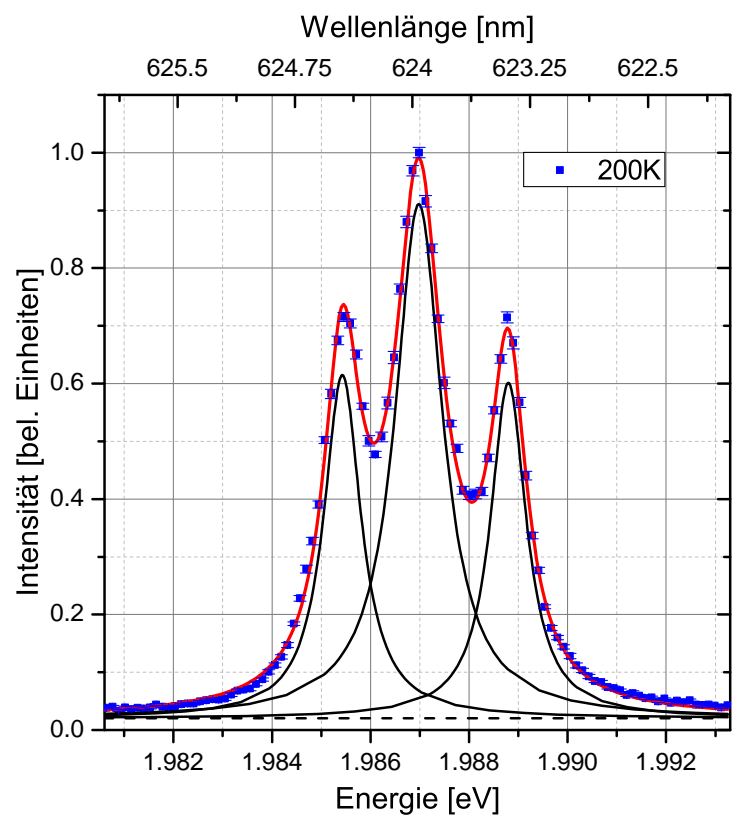

(c)

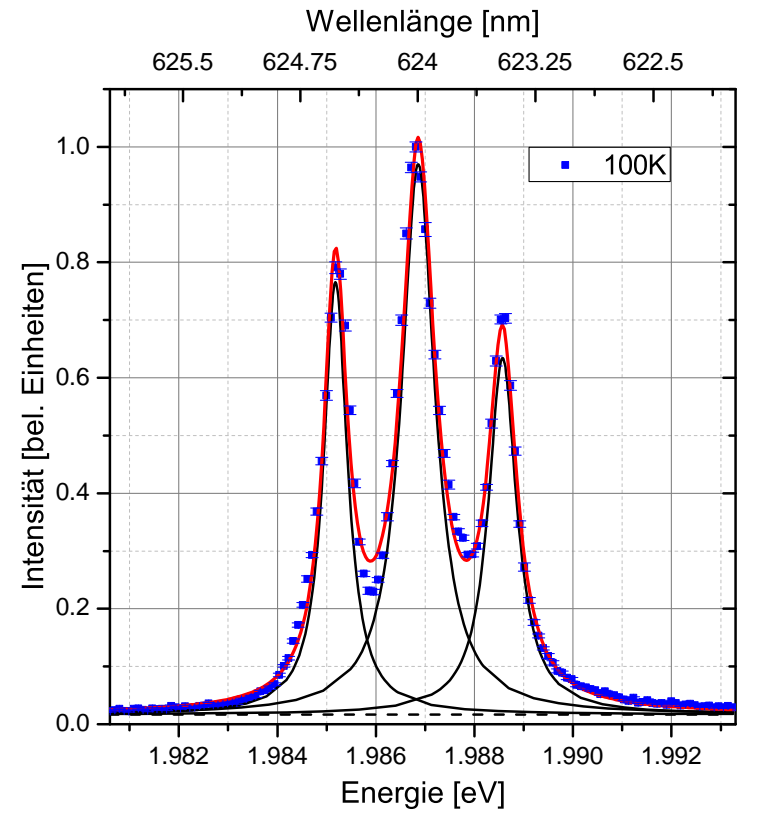

(b)

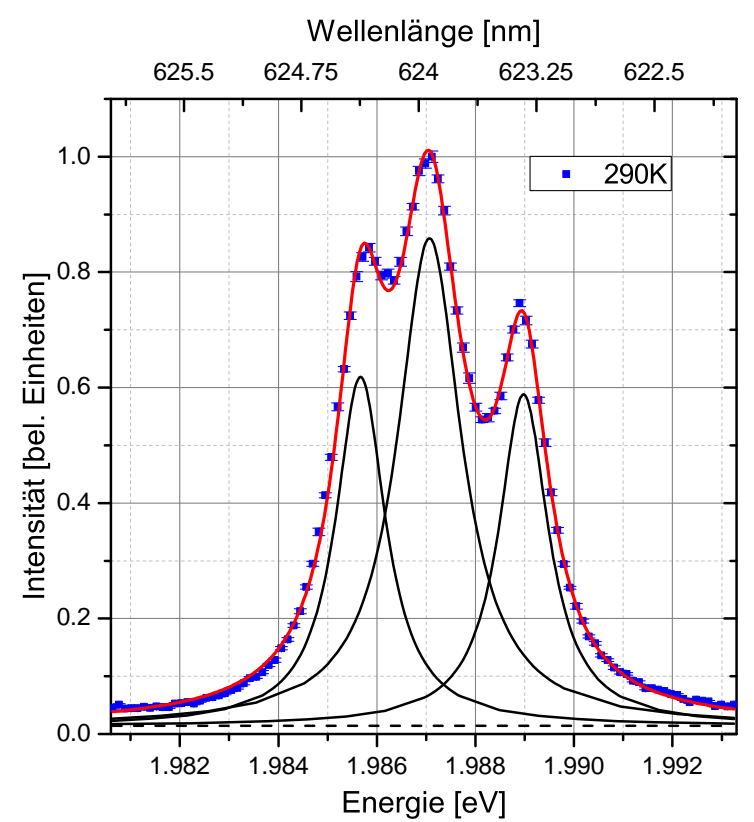

(d)

Abbildung 5.23: Anpassungen der Lumineszenz um $1.987 \mathrm{eV}$ mit drei Verteilungen nach Gleichung 5.7 zuzüglich Untergrund für vier Temperaturen. Für die Messungen unterhalb von $175 \mathrm{~K}$ als auch noch einmal für Messungen unterhalb von $75 \mathrm{~K}$ wurden jeweilig geringere Punktabstände für eine bessere Beschreibung der schmaler werdenden Bänder gewählt. 


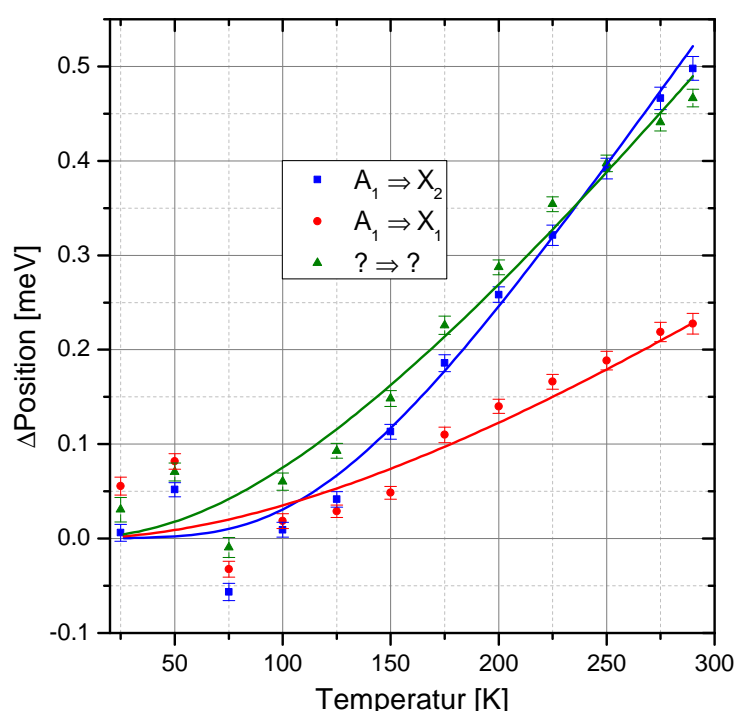

(a)

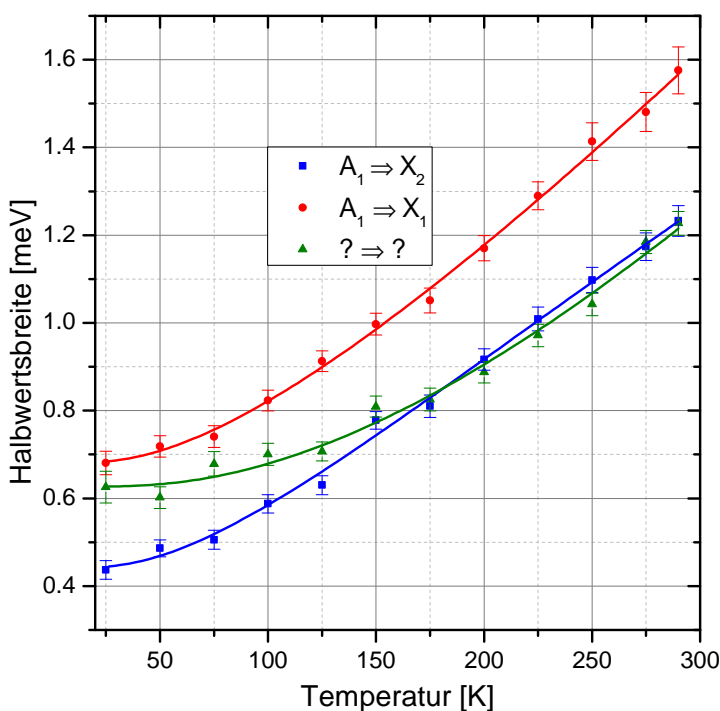

(b)

Abbildung 5.24: Verschiebung der Maxima (a) beziehungsweise die Änderung der Halbwertsbreite (b) der drei Lumineszenzbänder um $1.987 \mathrm{eV}$ im europiumdotierten AlN. Angepasst wurde jeweils für die Positionsänderung Gleichung A.19 und für die Breiten Gleichung A.20, Die Fehlerbalken der Datenpunkte resultieren aus den Unsicherheiten der Fits der jeweiligen Peaks.

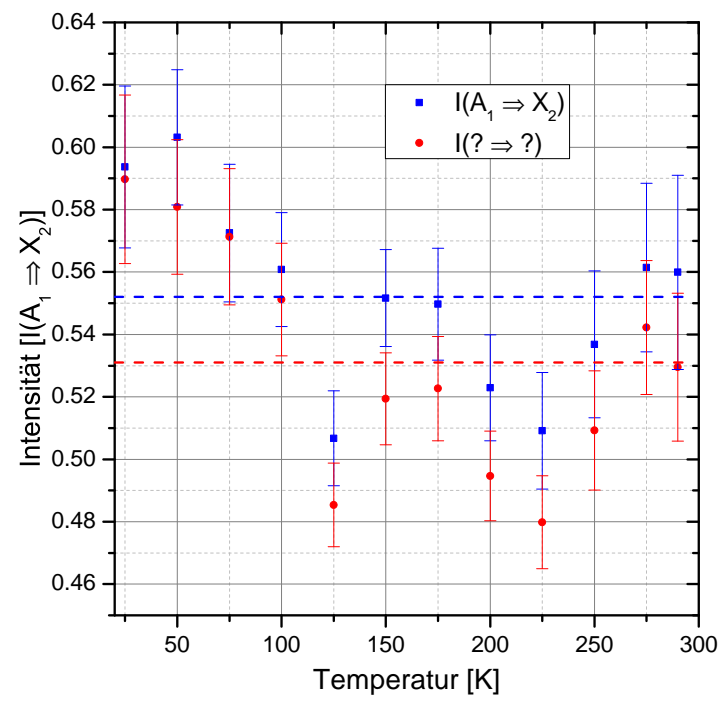

Abbildung 5.25: Temperaturabhängigkeit der Intensitäten der beiden Peaks im europiumdotierten AlN um die zentrale Lumineszenz bei $1.987 \mathrm{eV}$ normiert auf deren Wert. Die gestrichelten Geraden beschreiben den jeweiligen Mittelwert der Bänder. 
den beobachteten Transfers im freien Raum um verbotene Übergänge aufgrund der Paritätserhaltung handelt [222], diese Symmetrie jedoch durch das lokale Feld im Festkörper reduziert werden kann, ist von relativ langlebigen Übergängen auszugehen. In Abbildung 5.26 (a,b) sind zwei Übersichtsspektren aus Messungen mit Hilfe des Chopperrads (siehe Kapitel 3.3.5) für das ansteigende als auch das abfallende Lumineszenzsignal dargestellt. Dabei sind die drei schmalbandigen Lumineszenzzentren deutlich vom spektralen Untergrund zu unterscheiden. Eine Nahaufnahme der zuvor schon betrachteten Bänder um $1.987 \mathrm{eV}$ ist in Abbildung 5.26 (c,d) präsentiert. Die Anstiegs- als auch Abfallzeiten lassen sich innerhalb des $1 \mathrm{~ms}-Z$ Zeitfensters gut dokumentieren. Die drei Peaks um 1.987 eV besitzen im Rahmen der ermittelten Unsicherheiten gleiche Lebensdauern und werden nunmehr als ein Transfer behandelt, da zum einen bei höheren Temperaturen eine Trennung nur noch schwer möglich ist und zum andern die übrigen temperaturabhängigen Messungen mit dem 50er Gitter durchgeführt wurden, um jeweils immer alle drei Verläufe im Bereich von $1.85 \mathrm{eV}$ bis $2.1 \mathrm{eV}$ simultan registrieren zu können. Dies verhindert jedoch eine spektrale Aufösung von Unterstrukturen, wie die bei $1.987 \mathrm{eV}$.

Die Anstiegsflanken sowie auch die Zerfallstransienten wurden getrennt voneinander in verschiedenen Experimenten, wie in Abbildung 5.26 (a,b) dargestellt, aufgenommen und für die jeweiligen Peaks spektral integriert. Für eine Messung bei $290 \mathrm{~K}$ sind die daraus erhaltenen Intensitätsverläufe in Abbildung 5.27 gezeigt. In den dabei durchgeführten Anpassungen wurde ein direkter Transfer ohne Zwischenniveau angenommen (vergleiche Abbildung 4.11(a)). Der Grund liegt in schon vorher ermittelten Transferzeiten im Bereich weniger Mikrosekunden [282], welche aufgrund des groß gewählten Zeitfensters nicht mehr aufgelöst werden können und damit die Zahl der freien Parameter während des Fits unbegründet ansteigen lassen würde.

Die unter der Annahme lediglich einer Transferrate ermittelten Lebensdauern für die jeweiligen Anstiege und Zerfallstransienten sind in Abbildung 5.28 dargestellt. Da alle beobachteten Transfers von einem energetischen Niveau $\left({ }^{5} D_{0}\right)$ stammen sollen, ist mit einer einheitlichen Lebensdauer unabhängig der jeweiligen Linien zu rechnen. Insgesamt lässt sich für die jeweiligen Peaks keine eindeutige Abhängigkeit der Lebensdauer mit der Temperatur herstellen. Die jeweiligen Mittelwerte für die Anstiegs- und Abfallzeiten liegen jedoch nicht alle auf einem Wert zusammen. Insbesondere bei den Anstiegszeiten herrscht eine erhöhte Streuung der einzelnen Lebensdauern vor. Infolge der geringeren Intensitäten der Linien zu den Niveaus ${ }^{7} F_{1}$ und ${ }^{7} F_{3}$ spielt dort der spektrale Hintergrund, welcher ebenfalls verschiedene Lebensdauern besitzt, eine wesentlich größere Rolle als beim dominierenden Peak zum Niveau ${ }^{7} F_{2}$. Für jenen ist die durchschnittliche Anstiegszeit $\left(\tau_{r}=365(27) \mu \mathrm{s}\right)$ in ihrer Größe mit der Lebensdauer beim Abfall $\left(\tau_{d}=370(6) \mu \mathrm{s}\right)$, wie auch generell zu erwarten, vergleichbar. 


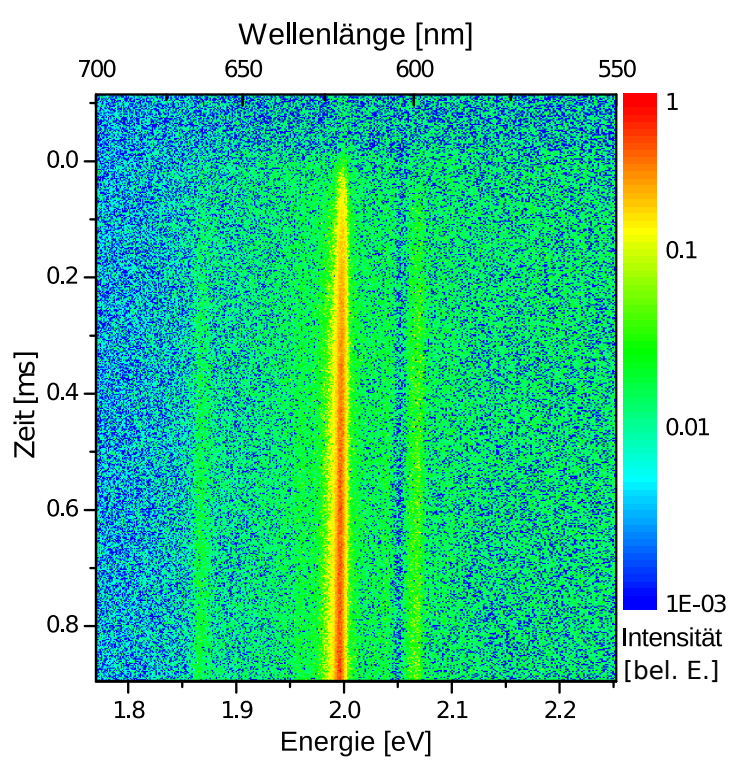

(a)

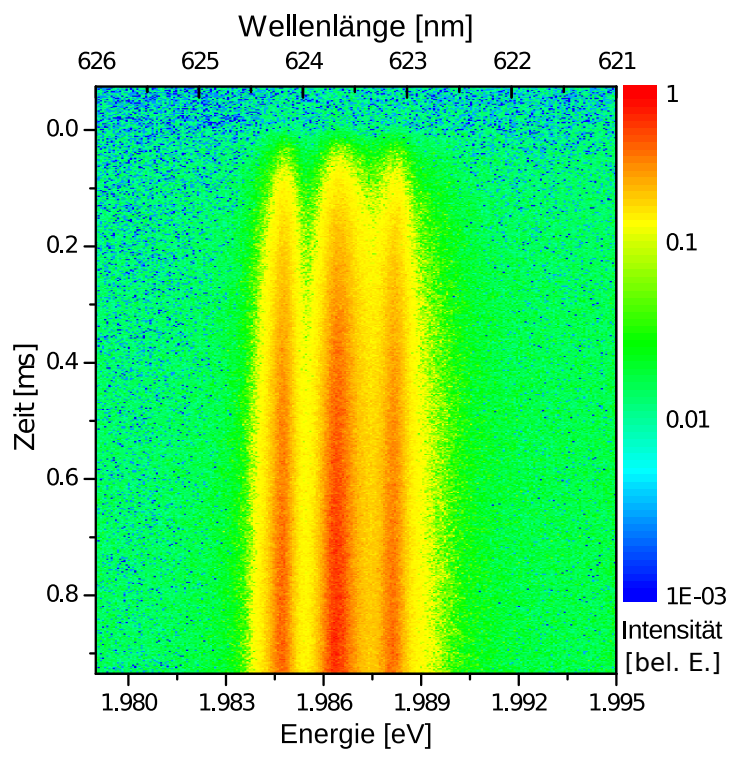

(c)

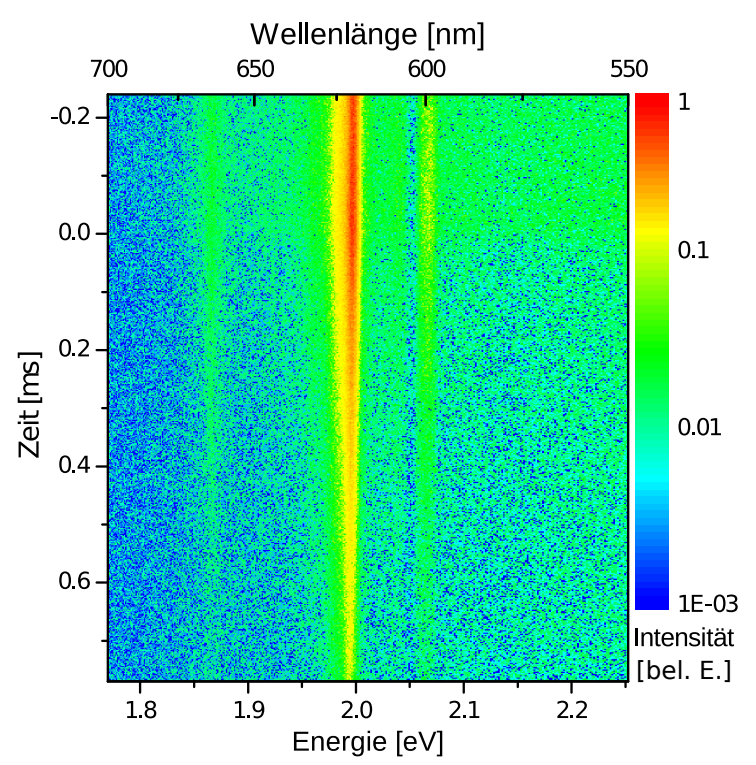

(b)

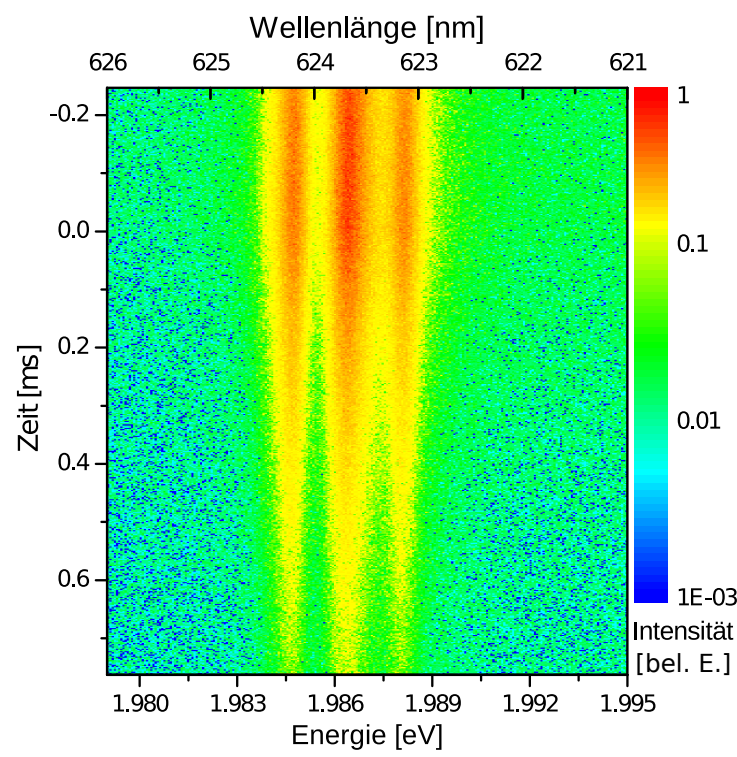

(d)

Abbildung 5.26: (a,b) Übersicht des zeitlichen Verhaltens der europiumspezifischen Lumineszenz bei sich öffnenden und schließenden Fenstern des Chopperrads bei einer Temperatur von $16 \mathrm{~K}$. Der lokale Intensitätsabfall bei $2.05 \mathrm{eV}$ stammt von einem Staubkorn am horizontalen Eingangsspalt der Streak-Kamera (siehe Kapitel 3.3.2). (c,d) Übersicht des zeitlichen Verhaltens der Lumineszenz direkt um $1.987 \mathrm{eV}$ mit einer hochaufgelösten Messung unter der Nutzung des 900er Gitters (siehe Kapitel 3.3.1. 


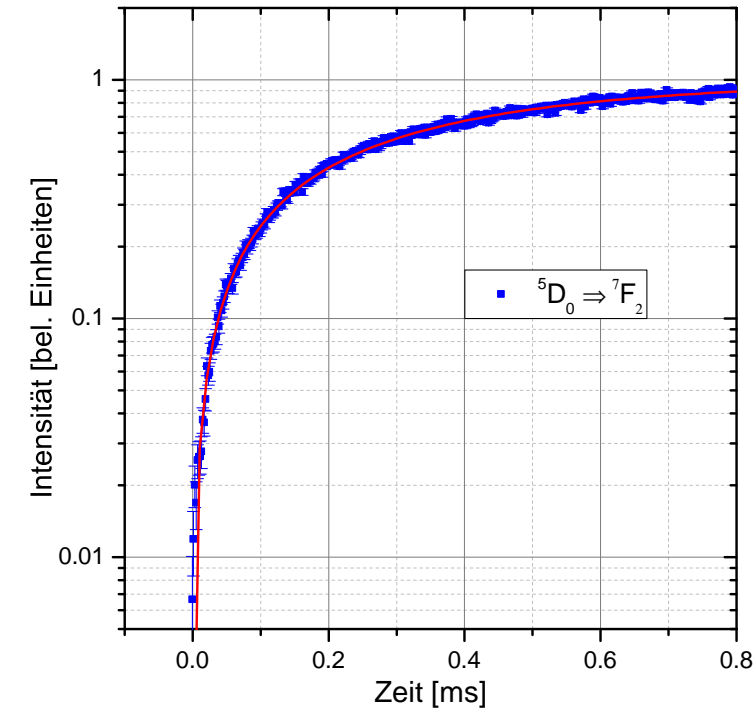

(a)

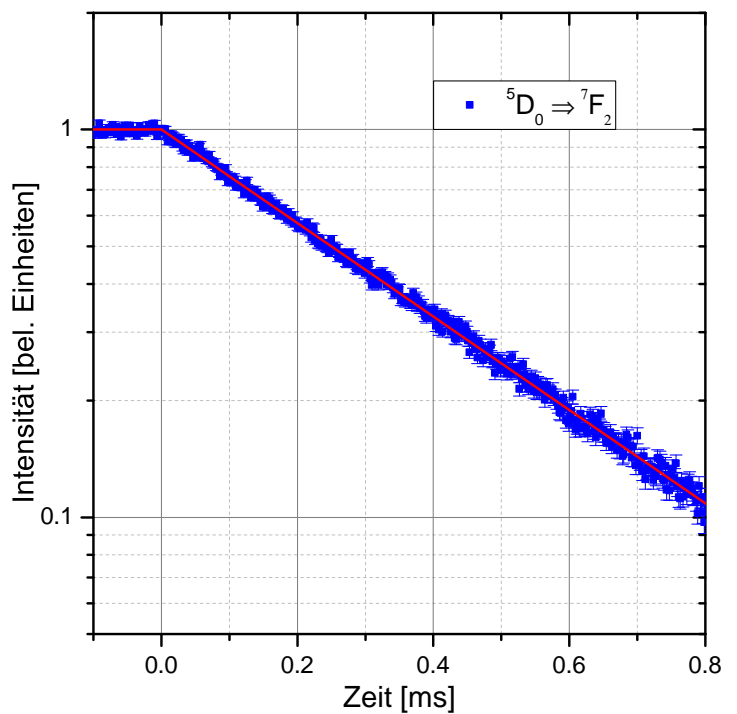

(b)

Abbildung 5.27: Ansteigendes (a) und abfallendes (b) Signal des europiumdotierten AlN integriert um den Bereich um $1.987 \mathrm{eV}$ bei einer Temperatur von $290 \mathrm{~K}$. Der Lumineszenzverlauf wurde jeweils durch die Gleichung A.4 beziehungsweise Gleichung A.7 beschrieben. Die Daten wurden dabei aus Messungen äquivalent zu denen in Abbildung 5.26 (a,b) gewonnen.

\subsubsection{Diskussion}

Das im Bereich um $2 \mathrm{eV}$ beobachtete aus schmalen Bändern bestehende Spektrum im dotierten AlN lässt sich eindeutig auf das seltenen Erd $E u^{3+}$ zurückführen. Die spektralen Eigenschaften passen sehr gut zu den in der Literatur angegebenen Werten (siehe Tabelle 2.5ID 39). Eine Beschreibung der einzelnen Peaks um $1.987 \mathrm{eV}$ als Lorentz-Funktionen führt über den gesamten betrachteten Temperaturbereich zu einer guten Anpassung. Lediglich zwischen den beiden niederenergetischen Signalen kann bei niedrigen Temperaturen eine signifikante Abweichung beobachtet werden. Jene könnte unter anderem aus der schon weiter oben beschriebenen endlichen Auflösung des spektroskopischen Aufbaus herrühren, wodurch für kleine Halbwertsbreiten eine künstliche Verbreiterung der Linien auftreten kann. Diese kann in etwa durch eine Faltung des Signals mit einer Normalverteilung beschrieben werden. Da jene für Werte abseits des Maximums signifikant schneller als eine Lorentz-Kurve abfällt, wird die tatsächliche Breite vor allem in Bereichen niedriger Intensitäten als zu groß abgeschätzt.

Die ermittelten Verschiebungen der Positionen der Maxima zeigen für die zwei niedrigsten Temperaturen eine dem Modell nicht entsprechende Abweichung. Dies kann aus einer Änderung der gemessenen Punktdichte für die zwei niedrigeren Tem- 


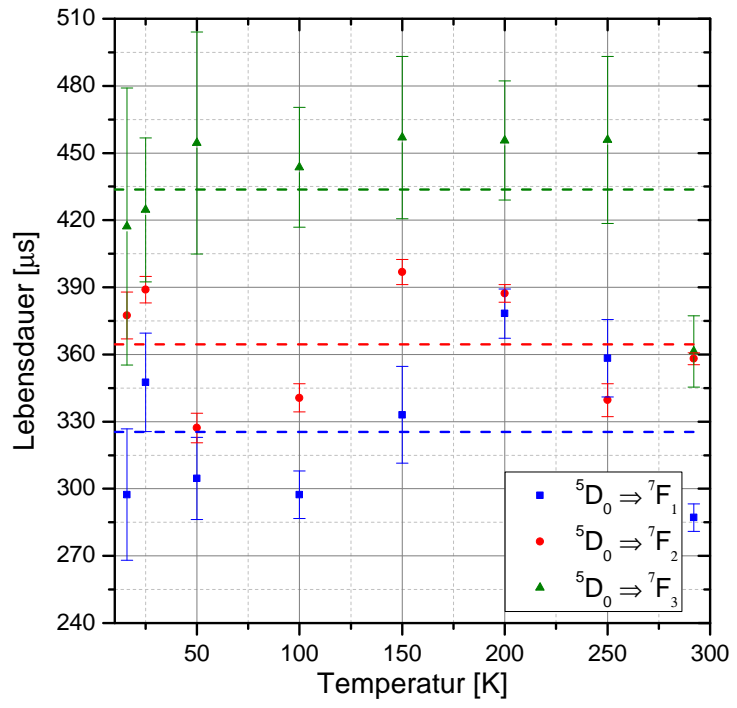

(a)

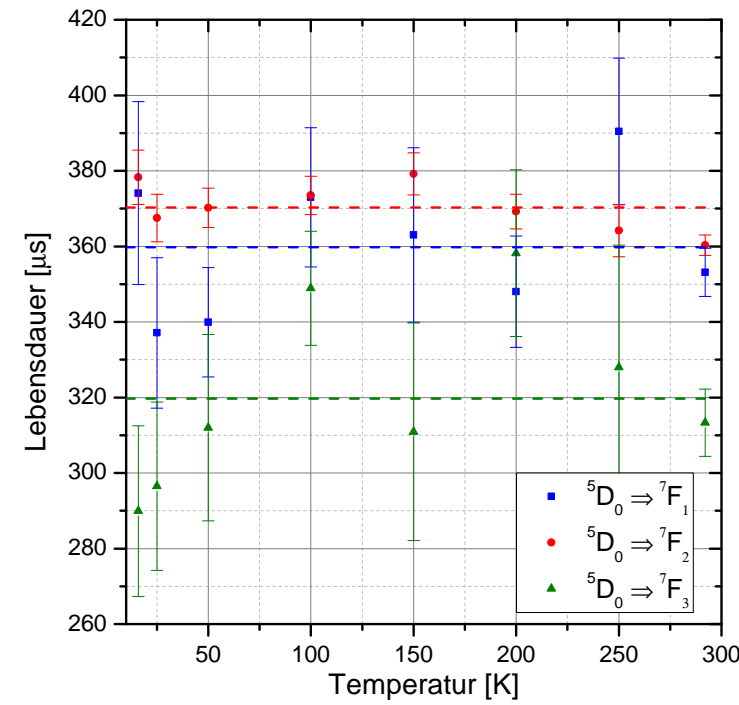

(b)

Abbildung 5.28: Lebensdauern ermittelt aus den Anstiegs- (a) als auch Abfallzeiten (b) für den betrachteten Temperaturbereich bis $290 \mathrm{~K}$. Die gestrichelten Linien beschreiben dabei den zur jeweiligen Farbe zugehörigen Mittelwert der Messdaten. Die dargestellten Fehler stammen aus den Unsicherheiten in den Anpassungen nach Gleichung A.4 beziehungsweise Gleichung A.7 für die bestimmten Parameter.

peraturen resultieren. Des weiteren ist zu beachten, dass sich die Änderungen der Positionen in der Größenordnung der spektralen Auflösung und darunter befinden, was ebenfalls stärkere Abweichungen erklären kann. Auf die Bestimmung der Halbwertsbreiten hat dies jedoch keinen erkennbaren Einfluss. Für die Verschiebungen und die Halbwertsbreiten ist festzuhalten, dass die durchgeführten Anpassungen nach den Gleichungen A.19 beziehungsweise A.20 aufgrund von vier frei verfügbaren Parametern hohe Unsicherheiten in der eigentlichen Bestimmung aufweisen. Die daraus ermittelbaren Aktivierungsenergien in einem Bereich um $10 \mathrm{meV}$ sind dabei sehr stark von den anderen freien Parametern abhängig.

Auch der bis jetzt nicht weiter zugeordnete Peak $(? \rightarrow$ ?) scheint einen unabhängigen Ursprung zu haben und nicht wie im Fall von Mangan auf der Grundlage eines Phononen-Replika zu basieren. Begründen lässt sich dies dadurch, dass sich seine relative Intensität im Verhältnis zu den beiden anderen Peaks mit der Temperatur nicht wesentlich verändert. Die beobachteten Varianzen und die damit einhergehenden Streuungen rühren wahrscheinlich von der ermittelten Intensität des Hauptmaximums her, da sich die beiden kleineren Peaks immer simultan zueinander verschieben.

Die ermittelten Lebensdauern beim Anstieg als auch beim Abfall der drei untersuchten Lumineszenzbänder sollten unter der Annahme eines einheitlichen Startni- 
veaus $\left({ }^{5} D_{0}\right)$ gleich sein. Die hohen Streuungen insbesondere bei den ansteigenden Zeiten können durch Ausläufer breitbandiger Defektbänder (siehe Kapitel 5.2) im Hintergrund des eigentlichen Signals erklärt werden. Für die Lebensdauermessung des stärksten Signals $\left({ }^{5} D_{0} \rightarrow{ }^{7} F_{2}\right)$ spielt dies aufgrund der hohen Intensität im Gegensatz zu den anderen Bändern bei den Zerfallstransienten nur eine untergeordnete Rolle. Die ermittelten Zeiten um $370 \mu$ s sind vergleichbar zu anderen in der Literatur gefundenen Werten [220,259]. Für den ansteigenden Teil ist das Modell eines einzelnen Übergangs gegebenenfalls zu einfach gewählt. Messungen an anderen seltenen Erd dotierten Systemen wie beispielsweise in Abbildung 4.14 durchgeführt, können bei kürzeren Lebensdauern besser von den langlebigen Untergründen separiert werden, was in diesem Fall nicht ohne weiteres möglich ist.

Auffällig für den nicht zugeordneten Transfer $(? \rightarrow$ ?) ist dessen gleiche Lebensdauer im Vergleich zu den zwei anderen bestimmten Übergängen. Dies lässt als oberes Niveau auch den Zustand ${ }^{5} D_{0}$ vermuten. 


\section{Zusammenfassung und Ausblick}

In diesem letzten Kapitel werden die Hauptkomponenten der Arbeit noch einmal zusammengefasst und ein Ausblick auf mögliche Verbesserungen im Aufbau oder bei den durchgeführten Messungen gegeben. Im Kern teilt sich diese Zusammenfassung dabei in die durchgeführte Literaturrecherche und deren Auswertung, den Aufbau und die Vermessung eines zeitabhängigen spektroskopischen Experiments sowie die Untersuchung HPHT-synthetisierter Proben.

\section{Lumineszenz in Aluminiumnitrid in der Literatur}

In Kapitel 2 konnte nach einer weitreichenden Literaturrecherche ein umfassender Überblick zu den in den letzten Jahrzehnten publizierten Artikeln, welche sich mit Lumineszenzen nahe der Bandkante sowie auch Lumineszenzen hervorgerufen durch verschiedenste Defekte in AlN beschäftigen, gegeben werden. Zusätzlich wurden neben den experimentellen Berichten auch theoretische Untersuchungen mit einem Fokus auf die Bandstruktur und die energetischen Positionen der Defektzustände analysiert. Die Ergebnisse wurden neben den beschreibenden Ausführungen in den Tabellen 2.4, 2.5 und A.1 übersichtlich zum schnellen Nachschlagen zusammengefasst. Wie anhand der Auswertung der vorhandenen Literatur zu erkennen ist, existiert eine Vielzahl von möglichen Ursachen für Lumineszenzen nahe der Bandkante und insbesondere tief innerhalb der Bandlücke.

Die Eigenschaften der freien Exzitonen in AlN sind prinzipiell gut verstanden. Lediglich das Vorzeichen der Konstante zur Beschreibung der Elektron-LochAustauschwechselwirkung ist zum jetzigen Zeitpunkt noch nicht abschließend geklärt. Auch die Energieverschiebungen durch das Einwirken einer mechanischen Spannung können gut erklärt werden. Im Gegensatz dazu sind für Exzitonen, welche an Donatoren gebunden sind in einem großen Teil der Fälle die mikroskopischen Ursachen unklar. Einzig die Exzitonen mit einer Bindung zu neutralen Siliziumoder Sauerstoffatomen wurden bis jetzt identifiziert. Für die an neutrale Akzeptoren gebundenen Exzitonen konnten aufgrund einer vorherigen Dotierung der Präparate einige Übergänge zugeordnet werden. Die damit eigentlich verbundene Intention, eine hinreichende p-Typ-Leitfähigkeit in Bulk-AlN bei Raumtemperatur zu erzielen, konnte bis heute nicht verwirklicht werden. Für hohe Anregungsintensitäten wird zudem in der Literatur von Rekombinationen infolge von Prozessen höherer Ordung, wie Bi-Exzitonen, Exziton-Exziton-Streuung als auch Elektron-Loch-Plasmen berichtet, welche ein superlineares intensitätsabhängies Verhalten zeigen. 
Defekte und Störstellen innerhalb des Materials sind der Ursprung einer großen Anzahl an verschiedenen Lumineszenzsignalen tief in der Bandlücke von AlN. Die meisten von ihnen zeigen eine starke Konzentrationsabhängigkeit dahingehend, ob sie unter Al-reichen oder N-reichen Bedingungen hergestellt wurden. Von der Vielzahl der möglichen intrinsischen Defekte wurden Leerstellen auf den Positionen des Aluminium- aber auch des Stickstoffplatzes in der Literatur sowohl theoretisch als auch experimentell am häufigsten untersucht. Dabei weisen sich Methoden, welche über die rein optischen Untersuchungen hinaus gehen, wie Positronen-AnnihilationsExperimente oder Elektronenspinresonanz-Messungen als ein nützliches Werkzeug zur Identifikation jener aus. Zusätzlich dazu können Experimente, bei denen sich die Proben nicht in thermischen Gleichgewichtsbedingungen befinden, z. B. durch die gezielte Produktion intrinsischer Defekte über eine Bestrahlung der Präparate mit hochenergetischen Ionen, auch jene untersucht werden, welche aufgrund der Herstellungsbedingungen sehr hohe Bildungsenergien besitzen.

Neben den Lumineszenzen durch rein intrinsische Defekte existiert auch eine große Anzahl an beobachteten optischen Übergängen, die Defekten mit Fremdatomen zugeordnet werden. In vielen hier analysierten Berichten werden verschiedenste Lumineszenzsignale zu Rekombinationen mit Sauerstoff oder auch Leerstellen-SauerstoffKomplexen in Verbindung gebracht (siehe Kapitel 2.2.2). Die energetischen Positionen sind dabei über einen weiten Bereich innerhalb der Bandlücke verteilt. Des weiteren zeigt sich in der Literatur eine uneinheitliche Zuordnung verschiedener Lumineszenzzentren insbesondere im Fall der Leerstellen-Sauerstoff-Komplexe. Hier teilt sich die Literatur in zwei in der Zuordnung komplementäre Lager. Eine zweite typischerweise ungewollte aber häufig beobachtete Verunreinigung von AlN ist Kohlenstoff. Verglichen zu Sauerstoff ist die Zuordnung der energetischen Positionen in der Literatur hier jedoch einheitlicher. Neben den ungewollten Verunreinigungen führt auch eine gezielte Dotierung mit Donatoren oder Akzeptoren zur Erzielung einer signifikanten elektrischen Leitfähigkeit ebenso zu spezifischen Lumineszenzen. Im Fall von Akzeptoren werden diese Übergänge zumeist als DAPs in Verbindung mit Stickstoffleerstellen interpretiert. Das Einfügen von Elementen der Metalle führt hingegen typischerweise zu relativ schmalbandigen Linien.

Auch theoretische Untersuchungen mit unterschiedlichsten Ansätzen, welche innerhalb der letzten vier Jahrzehnten veröffentlicht wurden, wurden aufgearbeitet und in Verbindung mit den dazugehörigen Experimenten gebracht. Schon die frühen Arbeiten lieferten eine gute Beschreibung der Valenzbandstruktur von AlN, litten aber entweder an einer signifikanten Unterschätzung (OPW, LDA, GGA) oder an einer bei weitem zu starken Überschätzung (HF) der Bandlücke. Daher sollten die Ergebnisse zu den energetischen Positionen von Defektzuständen aus solchen Simulationen mehr als Indikatoren, anstatt einer exakten Repräsentation des Experiments gesehen werden. Dies gilt insbesondere in den Fällen, in denen die Berechnungen auf Grundlage einer Zinkblendenstruktur basieren. Neuere Ansätze hingegen (HSE, B3LYP, LDA-1/2) können die experimentell beobachtete Bandlücke reproduzieren, wobei 
dies jedoch meist durch das Einstellen bestimmter Mischungsparameter forciert wird. Gleichwohl führt dies tendenziell dennoch zu einer präziseren Repräsentation der spezifischen Energielevel innerhalb der Bandlücke.

\section{Aufbau und Charakterisierung einer zeitaufgelösten Lumineszenzapparatur}

Im Rahmen dieser Arbeit wurde der bestehende Lumineszenzaufbau um ein Femtosekunden-Lasersystem und eine Streak-Kamera zur Kurzzeit-Lumineszenz-Spektroskopie vom nahen Infrarot bis in den nahen UV-Bereich erweitert. Dabei wurde das System im Laufe der Arbeit sukzessive mit zusätzlichen Komponenten, wie beispielsweise einem Chopperrad oder einem Filterrad vervollständigt. Auch wurden die einzelnen Komponenten so neu angeordnet, dass ein schneller Wechsel zwischen zeitunabhängigen als auch zeitabhängigen Versuchsreihen möglich ist. Insbesondere die Entwicklung eines speziellen Trigger-Generators (siehe Kapitel 3.3.5) erweitert die Funktionalität des Systems hin zur Analyse von Lebensdauern im Bereich mittlerer zweistelliger Millisekunden. Somit können mit einer Anlage Zerfallszeiten auf einer Skala von neun Größenordnungen mit gleichzeitiger spektraler Information analysiert werden. Durch den Entwurf eines neuen Probenhalters ist es zudem möglich, neben Festkörpern, auch Flüssigkeiten zu untersuchen.

Zusätzlich zum Aufbau und der Erweiterung wurden die signifikanten Leistungsparameter der Kernkomponenten der in Verwendung befindlichen Elemente (Pumplaser, Femtosekunden-Laser, Pulspicker, harmonischer Generator, Chopperrad, beide Spektrometer, Streak-Einheit) vermessen (in Teilen auch aus externen Messungen zusammengetragen), ausgewertet und die maximalen Effizienzen des Systems ermittelt. Lediglich die Parameter der erzeugten FHG konnten aufgrund einer nicht hoch genug liegenden absoluten Leistung jener und einer nur schwer zu entkoppelnden Intensität von den verarmten Restsignalen niedrigerer Harmonischer mit den vorhandenen Messsystemen nicht bestimmt werden.

Des weiteren wurde auch eine umfangreiche Software zur effizienten Auswertung und Nachverarbeitung der proprietären Daten aus dem Streak-System programmiert. Somit können unter anderem Sensitivitätsunterschiede der unterschiedlichen Messbereiche als auch der verwendeten Gitter sowie das zeitliche Wandern des Trigger-Signals während einer Messung zumindest in Teilen kompensiert werden.

Für eine weitere Steigerung der Funktionalität des komplexen Lumineszenzaufbaus können zum jetzigen Zeitpunkt für die Zukunft noch einige Ergänzungen vorgeschlagen werden. Um auch die FHG(3+1) für Systeme mit nicht all zu hoher Lichtausbeute, wie das hier behandelte AlN, besser einsetzen zu können, müsste jene über eine höhere Intensität verfügen. Inzwischen existiert ein leistungsstärkerer Pumplaser (Verdi G20, Coherent, Inc.) mit einer doppelten Ausgangsleistung verglichen zum vorhandenen System, welcher kompatibel zum Femtosekunden-Laser ist. Dadurch könnte eine deutliche Leistungssteigerung bezüglich der FHG(3+1) erzielt 
werden. Zudem existiert auch ein alternatives Spiegelsystem für den FemtosekundenLaser, welches den spektralen Stimmbereich nach oben als auch nach unten erweitert. Dadurch wäre es möglich die momentan noch vorhandenen Lücken im einstellbaren Anregungsspektrum vollends zu schließen und somit im optimalen Fall eine Anregung von etwa 200 - $1040 \mathrm{~nm}$ zu ermöglichen. Möglicherweise wäre für die meisten Anwendungen vielleicht auch eine Nutzung der im Laserkopf befindlichen Diode als eine Alternative zur extern verwendeten Trigger-Diode möglich, womit sich der damit in Verbindung hängende Justageaufwand erübrigen würde.

Im Bereich der Signalaufnahme könnten die chromatischen Aberrationen hervorgerufen durch das vor den Spektrometern befindliche Linsensystem mit dem Einsatz von paraboloiden Hohlspiegeln eliminiert werden. Dadurch würden gemessene Intensitäten unterschiedlicher Wellenlängen leichter vergleichbar. Um dies auch für alle Temperaturen zu ermöglichen, müsste die Probe selbst nachjustierbar sein. Um auch noch Zerfälle mit einer noch kürzeren Lebensdauer untersuchen zu können, würde sich der Einsatz der Synchroscan-Einheit (M10911-01, Coherent, Inc.) anbieten. Jene liefert zeitliche Auflösungen bis hin zu 1 ps. Des weiteren erhöht sich dabei die effektive Sensitivität des Streak-Systems für diese kurzen Zeiten um einen Faktor von 19, da jeder einzelne Anregungspuls mit in die Messung einfließen kann. Insbesondere bei niedrigen Anregungsleistungen (beispielsweise die FHG(3+1)) würde sich somit für kurzlebige Signale wie Bandkantenlumineszenzen ein deutlicher Sensitivitätsgewinn ergeben.

Eine das Labor betreffende mögliche Erweiterung wäre eine aktive Steuerung der Luftfeuchtigkeit, welche insbesondere im Sommer sehr hohe Werte annimmt und durch die vorhandenen Absorptionsbereiche zu einer Reduzierung der Anregungsleistung führt. Zudem könnten Fußmatten und Filter in der Lüftungsanlage für eine Reduktion der Partikel in der Luft und einer damit verringerten stetigen Kontamination der Optiken sorgen.

\section{Experimentelle Untersuchung von Aluminiumnitrid}

Die im Rahmen dieser Arbeit am detailliertesten untersuchten Systeme sind über das HPHT-Verfahren hergestellte AlN-Einkristalle. Diese zeigen ein vielfältiges Spektrum hervorgerufen durch verschiedene Defekte sowie auch im Fall der dotierten Proben eine ausgeprägte Lumineszenz des Europium. Die Symbiose von zeitunabhängigen Spektren mit zeitabhängigen Informationen liefert gerade bei einer großen Zahl sich überlagernder Defektbänder eine Möglichkeit der Trennung der einzelnen Bereiche. Die Lebensdauern der beobachteten Übergänge schöpfen hierbei die komplette Bandbreite der mit dem spektroskopischen Aufbau bestimmbaren Transferzeiten aus. Sie reichen dabei von etwa 30 ps im Fall der Lumineszenz um $3.9 \mathrm{eV}$, welche wahrscheinlich von Übergängen vom Leitungsband zu Kohlenstoff herrührt, bis hin zu $16 \mathrm{~ms}$ bei einer Energie um $2.7 \mathrm{eV}$, die auch mit Kohlenstoff in Verbindung stehen könnte. Dabei zeigt sich für das betrachtete Probensystem, 
dass für die breitbandigen Lumineszenzbänder stets mehrere mögliche Ursachen existieren, welche nur durch spektroskopische Aufnahmen nicht abschließend geklärt werden können, zumal auch in der Literatur für viele Transfers unterschiedliche Ansichten bestehen. Um diese vorhandenen Mehrdeutigkeiten zu reduzieren, währen beispielsweise äquivalent hergestellte Proben mit leicht unterschiedlichen stöchiometrischen Zusammensetzungen bezüglich der Fremdatome (bestimmbar möglicherweise durch Sekundärionen-Massenspektroskopie) nötig, um jeweilig deren Einfluss auf die Spektren zu untersuchen. Auch alternative Methoden wie die Positronen-Annihilations-Spektroskopie, insbesondere bei der Analyse von Leerstellenkonzentrationen, könnten einen weiteren Aufschluss geben. Jedoch ist dies mit einem sehr hohen experimentellen Aufwand verbunden.

Die relativ schmalbandigen Signale um $2.1 \mathrm{eV}$ in den undotierten Proben konnten eindeutig dem Mangan zugeordnet werden. Eine genaue Analyse der zusätzlichen Bänder konnte mit Hilfe der temperaturabhängigen Messungen jene als phononische Repliken erklären. Die zeitabhängigen Messungen zeigen einen kaskadierten Übergang mit mindestens einem Zwischenniveau. Der eigentliche strahlende Übergang erwies sich im Einklang mit der Literatur in seiner Lebensdauer als temperaturunabhängig.

Das mit Europium dotierte AlN weist die für $E u^{3+}$ typischen schmalen Lumineszenzbänder auf. Eine Analyse der Lebensdauer der in ihrer Intensität am stärksten vertretenen Übergänge liefert Rekombinationszeiten im Bereich von $370 \mu \mathrm{s}$. Dies steht im Einklang mit in anderen Systemen ermittelten Lebensdauern aus der Literatur. Auch zeigt sich für einen bis jetzt nicht zugeordneten Peak, dass jener mit hoher Wahrscheinlichkeit ebenfalls, wie die anderen hier untersuchten Signale, vom Niveau ${ }^{5} D_{0}$ ausgeht.

Die in der Literatur zu findenden zeitabhängigen Untersuchungen bezüglich Übergangszeiten in AlN, welche mit Streak-Systemen durchgeführt wurden, beschränken sich auf Lumineszenzen nahe der Bandkante. Dabei wurden nur die dort auftretenden Exzitonen mit ihren sehr kurzen Lebensdauern untersucht. Mit dem hier zusammengestellten Aufbau konnten jedoch Messungen auf verschiedensten Zeitskalen an AlN durchgeführt werden, was in diesem Umfang vorher (soweit bekannt) noch nicht geschehen ist. Dies lässt sich auch auf andere Systeme übertragen. Zum Beispiel die mit dem hier untersuchten verwandten wurtzitischen Halbleiter GaN oder $\mathrm{Al}_{x} \mathrm{Ga}_{1-x} \mathrm{~N}$ lassen sich hervorragend untersuchen. Aufgrund der einstellbaren Bandlücke im Fall des letzteren wären so gegebenenfalls auch Messungen oberhalb der Bandlücke unter der Nutzung der FHG(2+2) möglich.

Die hier untersuchten Proben wurden zwar im Rahmen der Arbeit schon weitgehend spektroskopisch analysiert, jedoch könnten zusätzliche Messungen mit anderen anregenden Wellenlängen als $\lambda_{e x}=267 \mathrm{~nm}$ durchgeführt werden, um unterschiedliche Absorptionszentren gezielt ansprechen zu können. Somit ist es wie bei einer Messung bei $\lambda_{e x}=377 \mathrm{~nm}$ schon gezeigt, möglich, beispielsweise langlebige Untergrundsignale zu unterdrücken, um die kurzlebigen Anteile besser untersuchen zu können. Des 
weiteren sind dadurch auch genauere Aussagen bezüglich der energetischen Lage der einzelnen Niveaus innerhalb der Bandlücke möglich. Jedoch erfordert dies einen enormen zusätzlichen Mess- als auch Auswertungsaufwand. 


\section{Literaturverzeichnis}

[1] F. Briegleb, A. Geuther, "Ueber das Stickstoffmagnesium und die Affinitäten des Stickgases zu Metallen", Ann. der Chemie und Pharm., 123(2):228, 1862

[2] J. W. Mallet, "XIII.-On aluminum nitride, and the action of metallic aluminum upon sodium carbonate at high temperatures", J. Chem. Soc., 30:349, 1876

[3] J. W. Mallet, "Ueber Stickstoffaluminium und die Einwirkung von Aluminium auf kohlensaures Natron bei hoher Temperatur", Justus Liebig's Ann. der Chemie, 186(1):155, 1877

[4] H. Ott, "Das Gitter des Aluminiumnitrids (AlN)", Zeitschrift für Phys., 22(1):201, 1924

[5] J. W. Richards, "The Serpek process for the manufacture of ammonium nitride", J. Ind. Eng. Chem., 5(4):335, 1913

[6] G. A. Slack, "Nonmetallic crystals with high thermal conductivity", J. Phys. Chem. Solids, 34(2):321, 1973

[7] G. A. Slack, R. Tanzilli, R. Pohl, J. Vandersande, "The intrinsic thermal conductivity of AIN", J. Phys. Chem. Solids, 48(7):641, 1987

[8] J. Edwards, K. Kawabe, G. Stevens, R. Tredgold, "Space charge conduction and electrical behaviour of aluminium nitride single crystals", Solid State Commun., 3(5):99, 1965

[9] G. A. Slack, S. F. Bartram, "Thermal expansion of some diamondlike crystals", J. Appl. Phys., 46(1):89, 1975

[10] D. Gerlich, S. L. Dole, G. A. Slack, "Elastic properties of aluminum nitride", J. Phys. Chem. Solids, 47(5):437, 1986

[11] J. Thorpe, K. Yen, "2.148 GHz surface acoustic wave oscillator", 1979 Ultrason. Symp., 882-885, 1979

[12] M. B. Assouar, O. Elmazria, P. Kirsch, P. Alnot, V. Mortet, C. Tiusan, "High-frequency surface acoustic wave devices based on AlN/diamond layered structure realized using e-beam lithography", J. Appl. Phys., 101(11):114507, 2007 
[13] F. Medjdoub, M. Zegaoui, D. Ducatteau, N. Rolland, P. A. Rolland, "Highperformance low-leakage-current AlN/GaN HEMTs grown on silicon substrate", IEEE Electron Device Lett., 32(7):874, 2011

[14] A. Bairamis, C. Zervos, A. Adikimenakis, A. Kostopoulos, M. Kayambaki, K. Tsagaraki, G. Konstantinidis, A. Georgakilas, "Electron density and currents of AlN/GaN high electron mobility transistors with thin GaN/AlN buffer layer", Appl. Phys. Lett., 105(11):113508, 2014

[15] Karl Sensse, "Aluminiumnitrid und seine durch Silicium bedingte Phosphoreszenz", Ph.D. thesis, Friedrich-Wilhelms-Universität zu Berlin, 1928

[16] E. Tiede, "Herstellung eines rotleuchtenden Aluminiumnitrid-ManganLuminophors (Patent Nr. 739390)", 1943

[17] A. BenMoussa, A. Soltani, U. Schühle, K. Haenen, Y. Chong, W. Zhang, R. Dahal, J. Lin, H. Jiang, H. Barkad, B. BenMoussa, D. Bolsee, C. Hermans, U. Kroth, C. Laubis, V. Mortet, J. De Jaeger, B. Giordanengo, M. Richter, F. Scholze, J. Hochedez, "Recent developments of wide-bandgap semiconductor based UV sensors", Diam. Relat. Mater., 18(5-8):860, 2009

[18] Y. Taniyasu, M. Kasu, T. Makimoto, "An aluminium nitride light-emitting diode with a wavelength of 210 nanometres", Nature, 441(7091):325, 2006

[19] S. Zhao, A. T. Connie, M. H. T. Dastjerdi, X. H. Kong, Q. Wang, M. Djavid, S. Sadaf, X. D. Liu, I. Shih, H. Guo, Z. Mi, "Aluminum nitride nanowire light emitting diodes: Breaking the fundamental bottleneck of deep ultraviolet light sources.", Sci. Rep., 5:8332, 2015

[20] J. B. Gruber, U. Vetter, H. Hofsäss, B. Zandi, M. F. Reid, "Spectra and energy levels of $G^{3+}\left(4 f^{7}\right)$ in AlN", Phys. Rev. B, 69(19):195202, 2004

[21] N. Nepal, M. L. Nakarmi, H. U. Jang, J. Y. Lin, H. X. Jiang, "Growth and photoluminescence studies of Zn-doped AlN epilayers", Appl. Phys. Lett., 89(19):192111, 2006

[22] M. L. Nakarmi, N. Nepal, C. Ugolini, T. M. Altahtamouni, J. Y. Lin, H. X. Jiang, "Correlation between optical and electrical properties of Mg-doped AlN epilayers", Appl. Phys. Lett., 89(15):152120, 2006

[23] Z. Y. Fan, J. Y. Lin, H. X. Jiang, "Achieving conductive high Al-content AlGaN alloys for deep UV photonics", M. Razeghi, G. J. Brown (Editors), "Integr. Optoelectron. Devices 2007", International Society for Optics and Photonics, 2007, 64791I-1 
[24] L. T. Berzina, Baiba, Advances in Ceramics - Characterization, Raw Materials, Processing, Properties, Degradation and Healing, InTech, 2011

[25] T. Tansley, R. Egan, "Point-defect energies in the nitrides of aluminum, gallium, and indium", Phys. Rev. B, 45(19):10942, 1992

[26] H. Morkoc, Handbook of Nitride Semiconductors and Devices, WILEY-VCH Verlag GmbH \& Co. KGaA, 2009

[27] D. Palmer, Comprehensive Semiconductor Science and Technology, Elsevier, 2011

[28] A. Sedhain, J. Lin, H. Jiang, Handbook of luminescent semiconductor materials, AlN: Properties and Applications, CRC Press, 2012

[29] T. Koppe, H. Hofsäss, U. Vetter, "Overview of band-edge and defect related luminescence in aluminum nitride", J. Lumin., 178:267, 2016

[30] L. Roskovcová, J. Pastrňák, "The "Urbach" absorption edge in ALN", Czechoslov. J. Phys., 30(5):586, 1980

[31] F. R. M. Hossain, X. Tang, K. Wongchotigul, M. G. Spencer, "Study of cathodoluminescence spectroscopy of aluminum nitride", D. K. DeBusk, R. T. Chen (Editors), "Microelectron. Manuf. 1996", International Society for Optics and Photonics, 1996, 42-45

[32] A. Sedhain, J. Y. Lin, H. X. Jiang, "Nature of optical transitions involving cation vacancies and complexes in AlN and AlGaN", Appl. Phys. Lett., 100(22):221107, 2012

[33] M. Bickermann, B. M. Epelbaum, M. Kazan, Z. Herro, P. Masri, A. Winnacker, "Growth and characterization of bulk AlN substrates grown by PVT", Phys. status solidi, 202(4):531, 2005

[34] M. Feneberg, R. a. R. Leute, B. Neuschl, K. Thonke, M. Bickermann, "Highexcitation and high-resolution photoluminescence spectra of bulk AlN", Phys. Rev. B, 82(7):075208, 2010

[35] M. Feneberg, M. Fátima Romero, B. Neuschl, K. Thonke, M. Röppischer, C. Cobet, N. Esser, M. Bickermann, R. Goldhahn, "Negative spin-exchange splitting in the exciton fine structure of AlN", Appl. Phys. Lett., 102(5):052112, 2013

[36] Z. Bryan, I. Bryan, M. Bobea, L. Hussey, R. Kirste, Z. Sitar, R. Collazo, "Exciton transitions and oxygen as a donor in m-plane AlN homoepitaxial films", J. Appl. Phys., 115(13):133503, 2014 
[37] I. Bryan, Z. Bryan, M. Bobea, L. Hussey, R. Kirste, R. Collazo, Z. Sitar, "Homoepitaxial AlN thin films deposited on m-plane ( 1100) AlN substrates by metalorganic chemical vapor deposition", J. Appl. Phys., 116(13):133517, 2014

[38] G. Rossbach, M. Feneberg, M. Röppischer, C. Werner, N. Esser, C. Cobet, T. Meisch, K. Thonke, A. Dadgar, J. Bläsing, A. Krost, R. Goldhahn, "Influence of exciton-phonon coupling and strain on the anisotropic optical response of wurtzite AlN around the band edge", Phys. Rev. B - Condens. Matter Mater. Phys., 83(19):195202, 2011

[39] L. Chen, B. J. Skromme, R. F. Dalmau, R. Schlesser, Z. Sitar, C. Chen, W. Sun, J. Yang, M. a. Khan, M. L. Nakarmi, J. Y. Lin, H. X. Jiang, "Bandedge exciton states in AlN single crystals and epitaxial layers", Appl. Phys. Lett., 85(19):4334, 2004

[40] E. Silveira, J. A. Freitas, O. J. Glembocki, G. A. Slack, L. J. Schowalter, "Excitonic structure of bulk AIN from optical reflectivity and cathodoluminescence measurements", Phys. Rev. B - Condens. Matter Mater. Phys., 71(4):041201, 2005

[41] A. Sedhain, J. Y. Lin, H. X. Jiang, "Valence band structure of AlN probed by photoluminescence", Appl. Phys. Lett., 92(4):041114, 2008

[42] T. Onuma, T. Shibata, K. Kosaka, K. Asai, S. Sumiya, M. Tanaka, T. Sota, A. Uedono, S. F. Chichibu, "Free and bound exciton fine structures in AlN epilayers grown by low-pressure metalorganic vapor phase epitaxy", J. Appl. Phys., 105(2):023529, 2009

[43] J. Li, K. B. Nam, M. L. Nakarmi, J. Y. Lin, H. X. Jiang, P. Carrier, S.-H. Wei, "Band structure and fundamental optical transitions in wurtzite AlN", Appl. Phys. Lett., 83(25):5163, 2003

[44] E. Kuokstis, J. Zhang, Q. Fareed, J. W. Yang, G. Simin, M. A. Khan, R. Gaska, M. Shur, C. Rojo, L. Schowalter, "Near-band-edge photoluminescence of wurtzite-type AlN", Appl. Phys. Lett., 81(15):2755, 2002

[45] E. Silveira, J. A. Freitas, G. A. Slack, L. J. Schowalter, "Cathodoluminescence studies of large bulk AlN crystals", Phys. status solidi, 0(7):2618, 2003

[46] E. Silveira, J. A. Freitas, M. Kneissl, D. W. Treat, N. M. Johnson, G. A. Slack, L. J. Schowalter, "Near-bandedge cathodoluminescence of an AlN homoepitaxial film", Appl. Phys. Lett., 84(18):3501, 2004 
[47] T. Onuma, S. F. Chichibu, T. Sota, K. Asai, S. Sumiya, T. Shibata, M. Tanaka, "Exciton spectra of an AlN epitaxial film on (0001) sapphire substrate grown by low-pressure metalorganic vapor phase epitaxy", Appl. Phys. Lett., 81(4):652, 2002

[48] S. Tungasmita, P. O. A. Persson, L. Hultman, J. Birch, "Pulsed low-energy ion-assisted growth of epitaxial aluminum nitride layer on $6 \mathrm{H}$-silicon carbide by reactive magnetron sputtering", J. Appl. Phys., 91(6):3551, 2002

[49] G. M. Prinz, A. Ladenburger, M. Schirra, M. Feneberg, K. Thonke, R. Sauer, Y. Taniyasu, M. Kasu, T. Makimoto, "Cathodoluminescence, photoluminescence, and reflectance of an aluminum nitride layer grown on silicon carbide substrate", J. Appl. Phys., 101(2):023511, 2007

[50] G. Prinz, A. Ladenburger, M. Feneberg, M. Schirra, S. Thapa, M. Bickermann, B. Epelbaum, F. Scholz, K. Thonke, R. Sauer, "Photoluminescence, cathodoluminescence, and reflectance study of AlN layers and AlN single crystals", Superlattices Microstruct., 40(4-6):513, 2006

[51] T. Onuma, K. Hazu, A. Uedono, T. Sota, S. F. Chichibu, "Identification of extremely radiative nature of AlN by time-resolved photoluminescence", Appl. Phys. Lett., 96(6):061906, 2010

[52] T. Koyama, M. Sugawara, T. Hoshi, A. Uedono, J. F. Kaeding, R. Sharma, S. Nakamura, S. F. Chichibu, "Relation between Al vacancies and deep emission bands in AlN epitaxial films grown by $\mathrm{NH}_{3}$-source molecular beam epitaxy", Appl. Phys. Lett., 90(24):241914, 2007

[53] T. Hoshi, T. Koyama, M. Sugawara, A. Uedono, J. F. Kaeding, R. Sharma, S. Nakamura, S. F. Chichibu, "Correlation between the violet luminescence intensity and defect density in AlN epilayers grown by ammonia-source molecular beam epitaxy", Phys. status solidi, 5(6):2129, 2008

[54] H. Ikeda, T. Okamura, K. Matsukawa, T. Sota, M. Sugawara, T. Hoshi, P. Cantu, R. Sharma, J. F. Kaeding, S. Keller, U. K. Mishra, K. Kosaka, K. Asai, S. Sumiya, T. Shibata, M. Tanaka, J. S. Speck, S. P. DenBaars, S. Nakamura, T. Koyama, T. Onuma, S. F. Chichibu, "Impact of strain on freeexciton resonance energies in wurtzite AlN", J. Appl. Phys., 102(12):123707, 2007

[55] C. Reich, M. Feneberg, V. Kueller, A. Knauer, T. Wernicke, J. Schlegel, M. Frentrup, R. Goldhahn, M. Weyers, M. Kneissl, "Excitonic recombination in epitaxial lateral overgrown AlN on sapphire", Appl. Phys. Lett., 103(21):212108, 2013 
[56] B. Neuschl, K. Thonke, M. Feneberg, S. Mita, J. Xie, R. Dalmau, R. Collazo, Z. Sitar, "Optical identification of silicon as a shallow donor in MOVPE grown homoepitaxial AlN", Phys. status solidi, 249(3):511, 2012

[57] M. Feneberg, B. Neuschl, K. Thonke, R. Collazo, A. Rice, Z. Sitar, R. Dalmau, J. Xie, S. Mita, R. Goldhahn, "Sharp bound and free exciton lines from homoepitaxial AlN", Phys. status solidi, 208(7):1520, 2011

[58] R. A. R. Leute, M. Feneberg, R. Sauer, K. Thonke, S. B. Thapa, F. Scholz, Y. Taniyasu, M. Kasu, "Photoluminescence of highly excited AlN: Biexcitons and exciton-exciton scattering", Appl. Phys. Lett., 95(3):031903, 2009

[59] Y. Taniyasu, M. Kasu, T. Makimoto, "Radiation and polarization properties of free-exciton emission from AlN (0001) surface", Appl. Phys. Lett., 90(26):261911, 2007

[60] B. N. Pantha, N. Nepal, T. M. Al Tahtamouni, M. L. Nakarmi, J. Li, J. Y. Lin, H. X. Jiang, "Correlation between biaxial stress and free exciton transition in AlN epilayers", Appl. Phys. Lett., 91(12):121117, 2007

[61] E. Silveira, J. Freitas, S. Schujman, L. Schowalter, "AlN bandgap temperature dependence from its optical properties", J. Cryst. Growth, 310(17):4007, 2008

[62] K. B. Nam, J. Li, M. L. Nakarmi, J. Y. Lin, H. X. Jiang, "Deep ultraviolet picosecond time-resolved photoluminescence studies of AlN epilayers", Appl. Phys. Lett., 82(11):1694, 2003

[63] J. Li, K. B. Nam, M. L. Nakarmi, J. Y. Lin, H. X. Jiang, "Band-edge photoluminescence of AlN epilayers", Appl. Phys. Lett., 81(18):3365, 2002

[64] B. Neuschl, K. Thonke, M. Feneberg, R. Goldhahn, T. Wunderer, Z. Yang, N. M. Johnson, J. Xie, S. Mita, A. Rice, R. Collazo, Z. Sitar, "Direct determination of the silicon donor ionization energy in homoepitaxial AlN from photoluminescence two-electron transitions", Appl. Phys. Lett., 103(12):122105, 2013

[65] R. M. Frazier, J. Stapleton, G. T. Thaler, C. R. Abernathy, S. J. Pearton, R. Rairigh, J. Kelly, A. F. Hebard, M. L. Nakarmi, K. B. Nam, J. Y. Lin, H. X. Jiang, J. M. Zavada, R. G. Wilson, "Properties of Co-, Cr-, or Mn-implanted AlN", J. Appl. Phys., 94(3):1592, 2003

[66] N. Nepal, K. B. Nam, M. L. Nakarmi, J. Y. Lin, H. X. Jiang, J. M. Zavada, R. G. Wilson, "Optical properties of the nitrogen vacancy in AlN epilayers", Appl. Phys. Lett., 84(7):1090, 2004 
[67] A. Sedhain, L. Du, J. H. Edgar, J. Y. Lin, H. X. Jiang, "The origin of 2.78 eV emission and yellow coloration in bulk AlN substrates", Appl. Phys. Lett., 95(26):262104, 2009

[68] N. Nepal, M. L. Nakarmi, K. B. Nam, J. Y. Lin, H. X. Jiang, "Acceptor-bound exciton transition in Mg-doped AlN epilayer", Appl. Phys. Lett., 85(12):2271, 2004

[69] H. Murotani, T. Kuronaka, Y. Yamada, T. Taguchi, N. Okada, H. Amano, "Temperature dependence of excitonic transitions in a-plane AlN epitaxial layers", J. Appl. Phys., 105(8):083533, 2009

[70] Y. Yamada, K. Choi, S. Shin, H. Murotani, T. Taguchi, N. Okada, H. Amano, "Photoluminescence from highly excited AlN epitaxial layers", Appl. Phys. Lett., 92(13):131912, 2008

[71] A. Sedhain, T. M. Al Tahtamouni, J. Li, J. Y. Lin, H. X. Jiang, "Beryllium acceptor binding energy in AlN", Appl. Phys. Lett., 93(14):141104, 2008

[72] J. A. Freitas, G. C. B. Braga, E. Silveira, J. G. Tischler, M. Fatemi, "Properties of Bulk AlN grown by thermodecomposition of $\mathrm{AlCl}_{3} \mathrm{xNH}_{3}$ ", Appl. Phys. Lett., 83(13):2584, 2003

[73] J. A. Freitas, "Optical studies of bulk and homoepitaxial films of III-V nitride semiconductors", J. Cryst. Growth, 281(1):168, 2005

[74] X. Tang, F. Hossain, K. Wongchotigul, M. G. Spencer, "Near band-edge transition in aluminum nitride thin films grown by metal organic chemical vapor deposition", Appl. Phys. Lett., 72(12):1501, 1998

[75] R. Kirste, M. R. Wagner, C. Nenstiel, F. Brunner, M. Weyers, A. Hoffmann, "Effect of TMGa preflow on the properties of high temperature AlN layers grown on sapphire", Phys. status solidi, 210(2):285, 2013

[76] W.-Y. Wang, P. Jin, G.-P. Liu, W. Li, B. Liu, X.-F. Liu, Z.-G. Wang, "Effect of high-temperature annealing on AlN thin film grown by metalorganic chemical vapor deposition", Chinese Phys. B, 23(8):087810, 2014

[77] A. Sedhain, N. Nepal, M. L. Nakarmi, T. M. Al tahtamouni, J. Y. Lin, H. X. Jiang, Z. Gu, J. H. Edgar, "Photoluminescence properties of AlN homoepilayers with different orientations", Appl. Phys. Lett., 93(4):041905, 2008

[78] A. Sedhain, J. Li, J. Y. Lin, H. X. Jiang, "Probing exciton-phonon interaction in AlN epilayers by photoluminescence", Appl. Phys. Lett., 95(6):061106, 2009 
[79] N. Teofilov, K. Thonke, R. Sauer, D. Ebling, L. Kirste, K. Benz, "Near bandedge transitions in AlN thin films grown on different substrates", Diam. Relat. Mater., 10(3-7):1300, 2001

[80] M. Funato, K. Matsuda, R. G. Banal, R. Ishii, Y. Kawakami, "Homoepitaxy and Photoluminescence Properties of (0001) AlN", Appl. Phys. Express, 5(8):082001, 2012

[81] S. F. Chichibu, K. Hazu, Y. Ishikawa, M. Tashiro, T. Ohtomo, K. Furusawa, A. Uedono, S. Mita, J. Xie, R. Collazo, Z. Sitar, "Excitonic emission dynamics in homoepitaxial AlN films studied using polarized and spatio-time-resolved cathodoluminescence measurements", Appl. Phys. Lett., 103(14):142103, 2013

[82] B. Hejda, "Energy Band Structure of AIN", Phys. status solidi, 32(1):407, 1969

[83] B. Hejda, K. Hauptmanová, "Energy Band Structure of AlN", Phys. status solidi, 36(2):K95, 1969

[84] S. Bloom, "Band structures of GaN and AIN", J. Phys. Chem. Solids, 32(9):2027, 1971

[85] D. Jones, A. Lettington, "The electronic band structures of the wide band gap semiconductors GaN and A1N", Solid State Commun., 11(5):701, 1972

[86] S.-H. Wei, A. Zunger, "Valence band splittings and band offsets of AlN, GaN, and InN", Appl. Phys. Lett., 69(18):2719, 1996

[87] X.-h. Jiang, J.-j. Shi, M. Zhang, H.-x. Zhong, P. Huang, Y.-m. Ding, T.-j. $\mathrm{Yu}, \mathrm{B}$. Shen, J. Lu, X. Wang, "Enhancement of TE polarized light extraction efficiency in nanoscale $(\mathrm{AlN})_{m} /(\mathrm{GaN})_{n}(m>n)$ superlattice substitution for Al-rich AlGaN disorder alloy: ultra-thin GaN layer modulation", New J. Phys., 16(11):113065, 2014

[88] W. M. Yim, "Epitaxially grown AlN and its optical band gap", J. Appl. Phys., 44(1):292, 1973

[89] S. K. Pugh, D. J. Dugdale, S. Brand, R. A. Abram, "Electronic structure calculations on nitride semiconductors", Semicond. Sci. Technol., 14(1):23, 1999

[90] L. Cláudio de Carvalho, A. Schleife, F. Fuchs, F. Bechstedt, "Valence-band splittings in cubic and hexagonal AlN, GaN, and InN", Appl. Phys. Lett., 97(23):232101, 2010 
[91] K. Kim, W. Lambrecht, B. Segall, M. van Schilfgaarde, "Effective masses and valence-band splittings in GaN and AlN", Phys. Rev. B, 56(12):7363, 1997

[92] J. A. Majewski, M. Städele, P. Vogl, "Electronic structure of biaxially strained wurtzite crystals GaN, AlN, and InN", MRS Internet J. Nitride Semicond. Res., 1:30, 1996

[93] S. L. Chuang, C. S. Chang, "k.p method for strained wurtzite semiconductors", Phys. Rev. B, 54(4):2491, 1996

[94] V. Fock, "Näherungsmethode zur Lösung des quantenmechanischen Mehrkörperproblems", Zeitschrift für Phys., 61(1-2):126, 1930

[95] J. Heyd, G. E. Scuseria, M. Ernzerhof, "Hybrid functionals based on a screened Coulomb potential", J. Chem. Phys., 118(18):8207, 2003

[96] R. Collazo, J. Xie, B. E. Gaddy, Z. Bryan, R. Kirste, M. Hoffmann, R. Dalmau, B. Moody, Y. Kumagai, T. Nagashima, Y. Kubota, T. Kinoshita, A. Koukitu, D. L. Irving, Z. Sitar, "On the origin of the $265 \mathrm{~nm}$ absorption band in AlN bulk crystals", Appl. Phys. Lett., 100(19):191914, 2012

[97] B. E. Gaddy, Z. Bryan, I. Bryan, R. Kirste, J. Xie, R. Dalmau, B. Moody, Y. Kumagai, T. Nagashima, Y. Kubota, T. Kinoshita, A. Koukitu, Z. Sitar, R. Collazo, D. L. Irving, "Vacancy compensation and related donor-acceptor pair recombination in bulk AlN", Appl. Phys. Lett., 103(16):161901, 2013

[98] B. E. Gaddy, Z. Bryan, I. Bryan, J. Xie, R. Dalmau, B. Moody, Y. Kumagai, T. Nagashima, Y. Kubota, T. Kinoshita, A. Koukitu, R. Kirste, Z. Sitar, R. Collazo, D. L. Irving, "The role of the carbon-silicon complex in eliminating deep ultraviolet absorption in AlN", Appl. Phys. Lett., 104(20):202106, 2014

[99] J. L. Lyons, a. Janotti, C. G. Van de Walle, "Effects of carbon on the electrical and optical properties of InN, GaN, and AlN", Phys. Rev. B, 89(3):035204, 2014

[100] Q. Yan, A. Janotti, M. Scheffler, C. G. Van de Walle, "Origins of optical absorption and emission lines in AlN", Appl. Phys. Lett., 105(11):111104, 2014

[101] E. O. Kane, "Band structure of indium antimonide", J. Phys. Chem. Solids, $1(4): 249,1957$

[102] J. Pastrňák, L. Roskovcová, "Optical absorption edge of AIN single crystals", Phys. Status Solidi, 26(2):591, 1968 
[103] H. Yamashita, K. Fukui, S. Misawa, S. Yoshida, "Optical properties of AlN epitaxial thin films in the vacuum ultraviolet region", J. Appl. Phys., 50(2):896, 1979

[104] K. B. Nam, J. Li, M. L. Nakarmi, J. Y. Lin, H. X. Jiang, "Unique optical properties of AlGaN alloys and related ultraviolet emitters", Appl. Phys. Lett., 84(25):5264, 2004

[105] I. Vurgaftman, J. R. Meyer, "Band parameters for nitrogen-containing semiconductors", J. Appl. Phys., 94(6):3675, 2003

[106] P. Tronc, Y. E. Kitaev, G. Wang, M. F. Limonov, A. G. Panfilov, G. Neu, "Optical Selection Rules for Hexagonal GaN", Phys. Status Solidi, 216(1):599, 1999

[107] R. Ishii, M. Funato, Y. Kawakami, "Huge electron-hole exchange interaction in aluminum nitride", Phys. Rev. B, 87(16):161204, 2013

[108] R. Ishii, M. Funato, Y. Kawakami, "Effects of strong electron-hole exchange and exciton-phonon interactions on the exciton binding energy of aluminum nitride", Jpn. J. Appl. Phys., 53(9):091001, 2014

[109] M. Leroux, S. Dalmasso, F. Natali, S. Helin, C. Touzi, S. Laügt, M. Passerel, F. Omnes, F. Semond, J. Massies, P. Gibart, "Optical characterization of $\mathrm{Al}_{x} \mathrm{Ga}_{1-x} \mathrm{~N}$ Alloys $(x<0.7)$ grown on sapphire or silicon", Phys. status solidi, 234(3):887, 2002

[110] B. N. Pantha, A. Sedhain, J. Li, J. Y. Lin, H. X. Jiang, "Probing the relationship between structural and optical properties of Si-doped AlN", Appl. Phys. Lett., 96(13):131906, 2010

[111] R. Dalmau, B. Moody, R. Schlesser, S. Mita, J. Xie, M. Feneberg, B. Neuschl, K. Thonke, R. Collazo, A. Rice, J. Tweedie, Z. Sitar, "Growth and characterization of AlN and AlGaN epitaxial films on AlN single crystal substrates", $J$. Electrochem. Soc., 158(5):H530, 2011

[112] N. Bohr, "On the constitution of atoms and molecules", Philos. Mag., 26(151):1, 1913

[113] B. Bastek, F. Bertram, J. Christen, T. Hempel, A. Dadgar, A. Krost, "Analysis of point defects in AlN epilayers by cathodoluminescence spectroscopy", Appl. Phys. Lett., 95(3):032106, 2009

[114] K. Kornitzer, W. Limmer, K. Thonke, R. Sauer, D. Ebling, L. Steinke, K. Benz, "AlN on sapphire and on SiC: CL and Raman study", J. Cryst. Growth, 201202:441, 1999 
[115] Y. Shishkin, R. Devaty, W. Choyke, "Near bandedge cathodoluminescence studies of AlN films: Dependence on MBE growth conditions", Phys. Stat. Sol., 594(2):591, 2001

[116] E. Monroy, J. Zenneck, G. Cherkashinin, O. Ambacher, M. Hermann, M. Stutzmann, M. Eickhoff, "Luminescence properties of highly Si-doped AlN", Appl. Phys. Lett., 88(7):071906, 2006

[117] M. Feneberg, N. T. Son, A. Kakanakova-Georgieva, "Exciton luminescence in AlN triggered by hydrogen and thermal annealing", Appl. Phys. Lett., 106(24):242101, 2015

[118] M. Hermann, F. Furtmayr, A. Bergmaier, G. Dollinger, M. Stutzmann, M. Eickhoff, "Highly Si-doped AlN grown by plasma-assisted molecular-beam epitaxy", Appl. Phys. Lett., 86(19):192108, 2005

[119] N. Nepal, M. Nakarmi, J. Lin, H. Jiang, "Photoluminescence studies of impurity transitions in AlGaN alloys", Appl. Phys. Lett., 89(9):092107, 2006

[120] G. M. Prinz, M. Feneberg, M. Schirra, R. Sauer, K. Thonke, S. B. Thapa, F. Scholz, "Silicon-doping induced strain of AlN layers: a comparative luminescence and Raman study", Phys. status solidi - Rapid Res. Lett., 2(5):215, 2008

[121] K. B. Nam, M. L. Nakarmi, J. Li, J. Y. Lin, H. X. Jiang, "Photoluminescence studies of Si-doped AlN epilayers", Appl. Phys. Lett., 83(14):2787, 2003

[122] I. Bryan, A. Rice, L. Hussey, Z. Bryan, M. Bobea, S. Mita, J. Xie, R. Kirste, R. Collazo, Z. Sitar, "Strain relaxation by pitting in AlN thin films deposited by metalorganic chemical vapor deposition", Appl. Phys. Lett., 102(6):061602, 2013

[123] D. W. Jenkins, J. D. Dow, "Electronic structures and doping of InN, $\mathrm{In}_{x} \mathrm{Ga}_{1-x} \mathrm{~N}$, and $\mathrm{In}_{x} \mathrm{Al}_{1-x} \mathrm{~N}^{\prime \prime}$, Phys. Rev. B, 39(5):3317, 1989

[124] C. Stampfl, C. G. Van de Walle, "Doping of $\mathrm{Al}_{x} \mathrm{Ga}_{1-x} \mathrm{~N}$ ", Appl. Phys. Lett., $72(4): 459,1998$

[125] C. G. Van de Walle, "DX -center formation in wurtzite and zinc-blende $\mathrm{Al}_{x} \mathrm{Ga}_{1-x} \mathrm{~N}^{\prime}$, 57(4):2033, 1998

[126] A. Fara, F. Bernardini, V. Fiorentini, "Theoretical evidence for the semiinsulating character of AlN", J. Appl. Phys., 85(3):2001, 1999 
[127] D. F. Hevia, C. Stampfl, F. Viñes, F. Illas, "Microscopic origin of n-type behavior in Si-doped AlN", Phys. Rev. B - Condens. Matter Mater. Phys., 88(8):085202, 2013

[128] C. H. Park, D. J. Chadi, "Stability of deep donor and acceptor centers in GaN, AlN, and BN", Phys. Rev. B, 55(19):12995, 1997

[129] T. Mattila, R. M. Nieminen, "Point-defect complexes and broadband luminescence in GaN and AlN", Phys. Rev. B, 55(15):9571, 1997

[130] P. Bogusławski, J. Bernholc, "Doping properties of C, Si, and Ge impurities in GaN and AlN", Phys. Rev. B, 56(15):9496, 1997

[131] R. Zeisel, M. W. Bayerl, S. T. B. Goennenwein, R. Dimitrov, O. Ambacher, M. S. Brandt, M. Stutzmann, "DX-behavior of Si in AlN", Phys. Rev. B, $61(24): 283,2000$

[132] S. Petit, R. Jones, M. J. Shaw, P. R. Briddon, B. Hourahine, T. Frauenheim, "Electronic behavior of rare-earth dopants in AlN: A density-functional study", Phys. Rev. B - Condens. Matter Mater. Phys., 72(7):073205, 2005

[133] N. T. Son, M. Bickermann, E. Janzén, "Silicon in AlN: shallow donor and DX behaviors", Phys. status solidi, 8(7-8):2167, 2011

[134] N. T. Son, M. Bickermann, E. Janzén, "Shallow donor and DX states of Si in AlN", Appl. Phys. Lett., 98(9):092104, 2011

[135] L. Silvestri, K. Dunn, S. Prawer, F. Ladouceur, "Hybrid functional study of Si and O donors in wurtzite AlN", Appl. Phys. Lett., 99(12):122109, 2011

[136] C. Stampfl, C. Van de Walle, "Theoretical investigation of native defects, impurities, and complexes in aluminum nitride", Phys. Rev. B, 65(15):155212, 2002

[137] T. Mattila, R. M. Nieminen, "Ab initio study of oxygen point defects in GaAs, GaN, and AlN.", Phys. Rev. B. Condens. Matter, 54(23):16676, 1996

[138] S. Yoshida, "Properties of $\mathrm{Al}_{x} \mathrm{Ga}_{1-x} \mathrm{~N}$ films prepared by reactive molecular beam epitaxy", J. Appl. Phys., 53(10):6844, 1982

[139] X. Zhang, P. Kung, A. Saxler, D. Walker, T. C. Wang, M. Razeghi, "Growth of $\mathrm{Al}_{x} \mathrm{Ga}_{1-x} \mathrm{~N}$ :Ge on sapphire and silicon substrates", Appl. Phys. Lett., 67(12):1745, 1995

[140] V. A. Soltamov, I. V. Ilyin, A. A. Soltamova, E. N. Mokhov, P. G. Baranov, "Identification of the deep level defects in AlN single crystals by electron paramagnetic resonance", J. Appl. Phys., 107(11):113515, 2010 
[141] S. Limpijumnong, C. G. Van De Walle, "Passivation and doping due to hydrogen in III-nitrides", Phys. Status Solidi Basic Res., 228(1):303, 2001

[142] J. R. Haynes, "Experimental proof of the existence of a new electronic complex in silicon", Phys. Rev. Lett., 4(7):361, 1960

[143] F. Mireles, S. E. Ulloa, "Acceptor binding energies in GaN and AlN", Phys. Rev. B, 58(7):3879, 1998

[144] R. Q. Wu, L. Shen, M. Yang, Z. D. Sha, Y. Q. Cai, Y. P. Feng, Z. G. Huang, Q. Y. Wu, "Possible efficient p-type doping of AlN using Be: An ab initio study", Appl. Phys. Lett., 91(15):152110, 2007

[145] Y. Zhang, W. Liu, H. Niu, "Native defect properties and p-type doping efficiency in group-IIA doped wurtzite AlN", Phys. Rev. B, 77(3):035201, 2008

[146] Á. Szabó, N. T. Son, E. Janzén, A. Gali, "Group-II acceptors in wurtzite AlN: A screened hybrid density functional study", Appl. Phys. Lett., 96(19):192110, 2010

[147] I. Gorczyca, A. Svane, N. Christensen, "Calculated defect levels in GaN and AlN and their pressure coefficients", Solid State Commun., 101(10):747, 1997

[148] I. Gorczyca, A. Svane, N. Christensen, "Theory of point defects in GaN, AlN, and BN: Relaxation and pressure effects", Phys. Rev. B, 60(11):8147, 1999

[149] R. Q. Wu, L. Shen, M. Yang, Z. D. Sha, Y. Q. Cai, Y. P. Feng, Z. G. Huang, Q. Y. Wu, "Enhancing hole concentration in AlN by Mg:O codoping: Ab initio study", Phys. Rev. B, 77(7):073203, 2008

[150] K. B. Nam, M. L. Nakarmi, J. Li, J. Y. Lin, H. X. Jiang, "Mg acceptor level in AlN probed by deep ultraviolet photoluminescence", Appl. Phys. Lett., 83(5):878, 2003

[151] A. Janotti, E. Snow, C. G. Van de Walle, "A pathway to p-type wide-band-gap semiconductors", Appl. Phys. Lett., 95(17):172109, 2009

[152] J. G. Tischler, J. A. Freitas, "Anharmonic decay of phonons in strain-free wurtzite AlN", Appl. Phys. Lett., 85(11):1943, 2004

[153] K. Huang, A. Rhys, "Theory of light absorption and non-radiative transitions in F-centres", Proc. R. Soc. London. Ser. A. Math. Phys. Sci., 204(1078):406, 1950

[154] M. A. Lampert, "Mobile and immobile effective-mass-particle complexes in nonmetallic solids", Phys. Rev. Lett., 1(12):450, 1958 
[155] L. Keldysh, A. Kozlov, "Collective properties of excitons in semiconductors", Sov. J. Exp. Theor. Phys., 27(3):521, 1968

[156] D. Magde, H. Mahr, "Exciton-exciton interaction in CdS, CdSe, and ZnO", Phys. Rev. Lett., 24(16):890, 1970

[157] M. Smith, G. D. Chen, J. Y. Lin, H. X. Jiang, A. Salvador, B. N. Sverdlov, A. Botchkarev, H. Morkoc, B. Goldenberg, "Mechanisms of band-edge emission in Mg-doped p-type GaN", Appl. Phys. Lett., 68(14):1883, 1996

[158] D. G. Thomas, J. J. Hopfield, W. M. Augustyniak, "Kinetics of radiative recombination at randomly distributed donors and acceptors", Phys. Rev., 140(1A):A 202, 1965

[159] R. Dingle, M. Ilegems, "Donor-acceptor pair recombination in GaN", Solid State Commun., 9(3):175, 1971

[160] K. Laaksonen, M. G. Ganchenkova, R. M. Nieminen, "Vacancies in wurtzite GaN and AlN.", J. Phys. Condens. Matter, 21(1):015803, 2009

[161] I. Gorczyca, N. Christensen, A. Svane, "Influence of hydrostatic pressure on cation vacancies in GaN, AlN, and GaAs", Phys. Rev. B, 66(7):075210, 2002

[162] A. Hung, S. P. Russo, D. G. McCulloch, S. Prawer, "An ab initio study of structural properties and single vacancy defects in wurtzite AlN.", J. Chem. Phys., 120(10):4890, 2004

[163] Q. Hu, T. Noda, H. Tanigawa, T. Yoneoka, S. Tanaka, "The oxygen-related defect complexes in AlN under gamma irradiation and quantum chemistry calculation", Nucl. Instruments Methods Phys. Res. Sect. B Beam Interact. with Mater. Atoms, 191(1-4):536, 2002

[164] H.-G. Ye, G.-D. Chen, Y.-Z. Zhu, H.-M. Lü, "First principle study of nitrogen vacancy in aluminium nitride", Chinese Phys., 16(12):3803, 2007

[165] P. Bogusławski, E. L. Briggs, J. Bernholc, "Amphoteric properties of substitutional carbon impurity in GaN and AlN", Appl. Phys. Lett., 69(2):233, 1996

[166] L. Ramos, J. Furthmüller, L. Scolfaro, J. Leite, F. Bechstedt, "Substitutional carbon in group-III nitrides: Ab initio description of shallow and deep levels", Phys. Rev. B, 66(7):075209, 2002

[167] C. G. Van de Walle, J. Neugebauer, "First-principles calculations for defects and impurities: Applications to III-nitrides", J. Appl. Phys., 95(8):3851, 2004 
[168] Y. Li, D. W. Brenner, "First principles prediction of the gas-phase precursors for AlN sublimation growth.", Phys. Rev. Lett., 92(7):075503, 2004

[169] Z. Wu, W. Zhang, H. Hu, S. Zuo, F. Wang, P. Yan, J. Wang, R. Zhuo, D. Yan, "Effect of temperature on growth and ultraviolet photoluminescence of Zn doped AlN nanostructures", Mater. Lett., 136:95, 2014

[170] Q. Hu, S. Tanaka, T. Yoneoka, V. Grismanovs, "In-situ luminescence measurement for AlN ceramics under reactor irradiation", Radiat. Eff. Defects Solids, 147(4):283, 1999

[171] Q. Hu, S. Tanaka, T. Yoneoka, T. Noda, "Study of radiation defects for AlN ceramics under $\mathrm{O}^{+}$irradiation", Nucl. Instruments Methods Phys. Res. Sect. $B$ Beam Interact. with Mater. Atoms, 166-167:70, 2000

[172] M. Bickermann, B. M. Epelbaum, O. Filip, B. Tautz, P. Heimann, A. Winnacker, "Faceting in AlN bulk crystal growth and its impact on optical properties of the crystals", Phys. Status Solidi, 9(3-4):449, 2012

[173] T. Nagashima, Y. Kubota, T. Kinoshita, Y. Kumagai, J. Xie, R. Collazo, H. Murakami, H. Okamoto, A. Koukitu, Z. Sitar, "Structural and optical properties of carbon-doped AlN substrates grown by hydride vapor phase epitaxy using AlN substrates prepared by physical vapor transport", Appl. Phys. Express, 5(12):125501, 2012

[174] A. Dadgar, A. Krost, J. Christen, B. Bastek, F. Bertram, A. Krtschil, T. Hempel, J. Bläsing, U. Haboeck, A. Hoffmann, "MOVPE growth of high-quality AlN", J. Cryst. Growth, 297(2):306, 2006

[175] A. Uedono, S. Ishibashi, S. Keller, C. Moe, P. Cantu, T. M. Katona, D. S. Kamber, Y. Wu, E. Letts, S. A. Newman, S. Nakamura, J. S. Speck, U. K. Mishra, S. P. DenBaars, T. Onuma, S. F. Chichibu, "Vacancy-oxygen complexes and their optical properties in AlN epitaxial films studied by positron annihilation", J. Appl. Phys., 105(5):054501, 2009

[176] R. A. Youngman, J. H. Harris, "Luminescence studies of oxygen-related defects In aluminum nitride", J. Am. Ceram. Soc., 73(11):3238, 1990

[177] K. B. Nam, M. L. Nakarmi, J. Y. Lin, H. X. Jiang, "Deep impurity transitions involving cation vacancies and complexes in AlGaN alloys", Appl. Phys. Lett., 86(22):222108, 2005

[178] M. Bickermann, B. M. Epelbaum, O. Filip, P. Heimann, S. Nagata, A. Winnacker, "Point defect content and optical transitions in bulk aluminum nitride crystals", Phys. status solidi, 246(6):1181, 2009 
[179] M. Bickermann, B. M. Epelbaum, A. Winnacker, "PVT growth of bulk AlN crystals with low oxygen contamination", Phys. status solidi, 0(7):1993, 2003

[180] B. Berzina, L. Trinkler, E. Palcevskis, J. Sils, "The oxygen-related luminescence centers in AlN ceramics", Proc. 13th Int. Conf. defects Insul. Mater. - ICDIM 96, 239-:145, 1997

[181] A. Sarua, S. Rajasingam, M. Kuball, N. Garro, O. Sancho, A. Cros, A. Cantarero, D. Olguin, B. Liu, D. Zhuang, J. H. Edgar, "Effect of impurities on raman and photoluminescence spectra of AlN bulk crystals", MRS Proc., 798:Y5.17, 2004

[182] M. Morita, K. Tsubouchi, N. Mikoshiba, "Optical absorption and cathodoluminescence of epitaxial aluminum nitride films", Jpn. J. Appl. Phys., 21(7):1102, 1982

[183] S. Yoshida, S. Misawa, S. Gonda, "Cathodoluminescence of impurity-doped aluminium nitride films produced by reactive evaporation", Thin Solid Films, $58(1): 55,1979$

[184] Q. Hu, S. Tanaka, T. Yoneoka, V. Grismanovs, T. Noda, "Aggregation of radiation defects in AIN ceramics under $\mathrm{He}^{+}$ion irradiation", Radiat. Eff. Defects Solids, 152(3):247, 2000

[185] U. Vetter, S. Müller, M. Brötzmann, H. Hofsäss, J. B. Gruber, "Effective reduction of AlN defect luminescence by fluorine implantation", Diam. Relat. Mater., 20(5-6):782, 2011

[186] I. Weinstein, A. Vokhmintsev, D. Spiridonov, "Thermoluminescence kinetics of oxygen-related centers in AlN single crystals", Diam. Relat. Mater., 25:59, 2012

[187] T. Schulz, M. Albrecht, K. Irmscher, C. Hartmann, J. Wollweber, R. Fornari, "Ultraviolet luminescence in AlN", Phys. status solidi, 248(6):1513, 2011

[188] D. Zhang, F.-M. Liu, L.-G. Cai, "N vacancy, substitutional O, and Al defects in the bandgap of composition-tunable nonstoichiometric AlN powder", Chinese Phys. B, 23(6):067803, 2014

[189] S. Bellucci, A. Popov, C. Balasubramanian, G. Cinque, A. Marcelli, I. Karbovnyk, V. Savchyn, N. Krutyak, "Luminescence, vibrational and XANES studies of AlN nanomaterials", Radiat. Meas., 42(4-5):708, 2007

[190] L. Shen, N. Wang, X. Xiao, "Strong orange luminescence from AlN whiskers", Mater. Lett., 94:150, 2013 
[191] L. Trinkler, B. Berzina, "Radiation induced recombination processes in AIN ceramics", J. Phys. Condens. Matter, 13(40):8931, 2001

[192] S. Schweizer, U. Rogulis, J.-M. Spaeth, L. Trinkler, B. Berzina, "Investigation of Oxygen-Related Luminescence Centres in AlN Ceramics", Phys. status solidi, 219(1):171, 2000

[193] L. Trinkler, B. Berzina, "Recombination luminescence in aluminum nitride ceramics", Phys. status solidi, 251(3):542, 2014

[194] B. Berzina, L. Trinkler, J. Sils, K. Atobe, "Luminescence mechanisms of oxygen-related defects in AlN", Radiat. Eff. Defects Solids, 157(6-12):1089, 2002

[195] B. Berzina, L. Trinkler, J. Sils, E. Palcevskis, "Oxygen-related defects and energy accumulation in aluminum nitride ceramics", Radiat. Eff. Defects Solids, 156(1-4):241, 2001

[196] B. Berzina, "Spectral characterization of bulk and nanostructured aluminum nitride", J. Nanophotonics, 3(1):031950, 2009

[197] J. Pastrňák, S. Pačesová, L. Roskovcová, "Luminescent properties of the oxygen impurity centres in AlN", Czechoslov. J. Phys., 24(10):1149, 1974

[198] J. Nappé, M. Benabdesselam, P. Grosseau, B. Guilhot, "Effect of swift heavy ion irradiations in polycrystalline aluminum nitride", Nucl. Instruments Methods Phys. Res. Sect. B Beam Interact. with Mater. Atoms, 269(2):100, 2011

[199] M. Benabdesselam, P. Iacconi, "Thermoluminescence of AlN. Influence of synthesis processes", J. Phys. Chem., 99(25):10319, 1995

[200] Y. Cao, X. Chen, Y. Lan, J. Li, Y. Xu, T. Xu, Q. Liu, J. Liang, "Blue emission and Raman scattering spectrum from AlN nanocrystalline powders", J. Cryst. Growth, 213(1-2):198, 2000

[201] A. L. Martin, C. M. Spalding, V. I. Dimitrova, P. G. Van Patten, M. L. Caldwell, M. E. Kordesch, H. H. Richardson, "Visible emission from amorphous AlN thin-film phosphors with $\mathrm{Cu}, \mathrm{Mn}$, or Cr", J. Vac. Sci. Technol. A Vacuum, Surfaces, Film., 19(4):1894, 2001

[202] N. Hirosaki, R.-J. Xie, K. Inoue, T. Sekiguchi, B. Dierre, K. Tamura, "Blueemitting AlN: $\mathrm{Eu}^{2+}$ nitride phosphor for field emission displays", Appl. Phys. Lett., 91(6):061101, 2007 
[203] B. Dierre, X. L. Yuan, K. Inoue, N. Hirosaki, R.-J. Xie, T. Sekiguchi, "Role of Si in the luminescence of AlN:Eu,Si phosphors", J. Am. Ceram. Soc., 92(6):1272, 2009

[204] H.-S. Do, S.-W. Choi, S.-H. Hong, "Blue-emitting AlN:Eu ${ }^{2+}$ powder phosphor prepared by spark plasma sintering", J. Am. Ceram. Soc., 93(2):356, 2010

[205] K. Inoue, N. Hirosaki, R.-J. Xie, T. Takeda, "Highly efficient and thermally stable blue-emitting AlN:Eu ${ }^{2+}$ phosphor for ultraviolet white light-emitting diodes", J. Phys. Chem. C, 113(21):9392, 2009

[206] T. Takeda, N. Hirosaki, R.-J. Xie, K. Kimoto, M. Saito, "Anomalous Eu layer doping in Eu, Si co-doped aluminium nitride based phosphor and its direct observation", J. Mater. Chem., 20(44):9948, 2010

[207] L.-J. Yin, Q.-Q. Zhu, W. Yu, L.-Y. Hao, X. Xu, F.-C. Hu, M.-H. Lee, "Europium location in the AlN: Eu green phosphor prepared by a gas-reductionnitridation route", J. Appl. Phys., 111(5):053534, 2012

[208] B. Dierre, X. M. Zhang, N. Fukata, T. Sekiguchi, T. Suehiro, T. Takeda, R. J. Xie, N. Hirosaki, "Growth temperature influence on the luminescence of Eu,Si-codoped AlN phosphors", ECS J. Solid State Sci. Technol., 2(7):R126, 2013

[209] H. Hu, X. Ji, Z. Wu, P. Yan, H. Zhou, S. Du, X. Wu, G. Gong, C. Li, "Synthesis and photoluminescence of AlN:Mn hexagonal maze-like complex nanostructure", Mater. Lett., 70:34, 2012

[210] T. Miyajima, Y. Kudo, T. Uruga, K. Hara, "Analysis of the local structure of AlN:Mn using X-ray absorption fine structure measurements", Phys. status solidi, 3(6):1742, 2006

[211] A. Sato, K. Azumada, T. Atsumori, K. Hara, "Characterization of AlN:Mn thin film phosphors prepared by metalorganic chemical vapor deposition", $J$. Cryst. Growth, 298:379, 2007

[212] F. Karel, J. Pastrňák, "Fine structure of emission spectra of the red AIN:Mn luminescence", Phys. status solidi, 693:693, 1966

[213] F. Karel, J. Mareš, "Electron states of manganese luminescence centres in AlN", Czechoslov. J. Phys. B, 22:847, 1972

[214] G. A. Wolff, I. Adams, J. W. Mellichamp, "Electroluminescence of AlN", Phys. Rev., 114(5):1262, 1959 
[215] C. Thomas, C. Taylor, J. Griffin, W. L. Rose, M. G. Spencer, M. Capano, S. Rendakova, K. Kornegay, "Annealing of ion implantation damage in $\mathrm{SiC}$ using a graphite mask", MRS Proc., 572:45, 1999

[216] M. Caldwell, H. Richardson, M. Kordesch, "Optical Properties of Manganese Doped Amorphous and Crystalline Aluminum Nitride Films", MRS Proc., 595:1, 1999

[217] I. Adams, T. R. AuCoin, G. A. Wolff, "Luminescence in the system $\mathrm{Al}_{2} \mathrm{O}_{3}$ AIN", J. Electrochem. Soc., 109(11):1050, 1962

[218] K. Hara, A. Sato, K. Azumada, T. Atsumori, M. Shiratori, "Preparation of AlN:Mn films by metalorganic chemical vapor deposition for thin film electroluminescent devices", Phys. status solidi, (7):2274, 2003

[219] A. Sato, K. Azumada, T. Atsumori, K. Hara, "Low-temperature metalorganic chemical vapor deposition of luminescent manganese-doped aluminum nitride films", Appl. Phys. Lett., 87(2):021907, 2005

[220] W. M. Jadwisienczak, H. J. Lozykowski, I. Berishev, A. Bensaoula, I. G. Brown, "Visible emission from AlN doped with Eu and Tb ions", J. Appl. Phys., 89(8):4384, 2001

[221] F. Liu, H. Dong, Q. Liu, J. Liang, J. Luo, Y. Zhang, L. Yang, G. Rao, "Characterization and photoluminescence of AlN:Eu films", Opt. Mater. (Amst)., 28(8):1029, 2006

[222] Y. Nakanishi, A. Wakahara, H. Okada, A. Yoshida, T. Ohshima, H. Itoh, T. Shibata, M. Tanaka, "Effects of Al composition on luminescence properties of europium implanted $\mathrm{Al}_{x} \mathrm{Ga}_{1-x} \mathrm{~N}(0 \leq x \leq 1)$ ", Phys. status solidi, $0(7): 2623$, 2003

[223] K. Lorenz, E. Alves, T. Monteiro, A. Cruz, M. Peres, "Structural and optical characterisation of Eu implanted $\mathrm{Al}_{x} \mathrm{Ga}_{1-x} \mathrm{~N}$ ", Nucl. Instruments Methods Phys. Res. Sect. B Beam Interact. with Mater. Atoms, 257(1):307, 2007

[224] B. Han, K. C. Mishra, M. Raukas, K. Klinedinst, J. Tao, J. B. Talbot, "A study of luminescence from $\mathrm{Tm}^{3+}, \mathrm{Tb}^{3+}$, and $\mathrm{Eu}^{3+}$ in AlN powder", J. Electrochem. Soc., 154(9):J262, 2007

[225] H. J. Lozykowski, W. M. Jadwisienczak, "Thermal quenching of luminescence and isovalent trap model for rare-earth-ion-doped AlN", Phys. Status Solidi Basic Res., 244(6):2109, 2007 
[226] K. Lorenz, E. Alves, F. Gloux, P. Ruterana, M. Peres, A. J. Neves, T. Monteiro, "Optical doping and damage formation in AlN by Eu implantation", J. Appl. Phys., 107(2):023525, 2010

[227] K. Lorenz, S. Magalhães, E. Alves, M. Peres, T. Monteiro, A. Neves, M. Boćkowski, "High temperature annealing of europium implanted AlN", Nucl. Instruments Methods Phys. Res. Sect. B Beam Interact. with Mater. Atoms, 268(19):2907, 2010

[228] J. B. Gruber, U. Vetter, T. Taniguchi, G. W. Burdick, H. Hofsäss, S. Chandra, D. K. Sardar, "Spectroscopic analysis of $\mathrm{Eu}^{3+}$ in single-crystal hexagonal phase AlN", J. Appl. Phys., 110(2):023104, 2011

[229] H. Lozykowski, W. Jadwisienczak, A. Bensaoula, O. Monteiro, "Luminescence and excitation mechanism of $\mathrm{Pr}, \mathrm{Eu}, \mathrm{Tb}$ and $\mathrm{Tm}$ ions implanted into AlN", Microelectronics J., 36(3):453, 2005

[230] J. Baur, M. Kunzer, K. Maier, U. Kaufmann, J. Schneider, "Determination of the GaN/AlN band discontinuities via the (-/0) acceptor level of iron", Mater. Sci. Eng. B, 29(1-3):61, 1995

[231] M. Bickermann, B. M. Epelbaum, O. Filip, P. Heimann, S. Nagata, A. Winnacker, "UV transparent single-crystalline bulk AlN substrates", Phys. Status Solidi Curr. Top. Solid State Phys., 7(1):21, 2010

[232] G. A. Slack, L. J. Schowalter, D. Morelli, J. A. Freitas, "Some effects of oxygen impurities on AlN and GaN", J. Cryst. Growth, 246(3-4):287, 2002

[233] G. Cox, D. Cummins, K. Kawabe, R. Tredgold, "On the preparation, optical properties and electrical behaviour of aluminium nitride", J. Phys. Chem. Solids, 28(4):543, 1967

[234] Q. Zhou, M. O. Manasreh, M. Pophristic, S. Guo, I. T. Ferguson, "Observation of nitrogen vacancy in proton-irradiated $\mathrm{Al}_{x} \mathrm{Ga}_{1-x} \mathrm{~N}$ ", Appl. Phys. Lett., 79(18):2901, 2001

[235] K. Atobe, M. Honda, N. Fukuoka, M. Okada, M. Nakagawa, "F-type centers in neutron-irradiated AIN", Jpn. J. Appl. Phys., 29(Part 1, No. 1):150, 1990

[236] D. Chen, J. Wang, D. Xu, Y. Zhang, "The influence of defects and impurities in polycrystalline AlN films on the violet and blue photoluminescence", Vacuum, 83(5):865, 2009

[237] X. Sun, J. Xiong, W. Zhang, L. Liu, H. Gu, "Investigation of blue luminescence in Mg doped AlN films", J. Alloys Compd., 621:314, 2015 
[238] Z. Yao, Y. Li, J. Tang, W. Zhang, S. Lee, "Growth and photoluminescence studies of AlN thin films with different orientation degrees", Diam. Relat. Mater., 17(7-10):1785, 2008

[239] J. Rosa, I. Tale, "Mechanism of thermoluminescence in AlN:O", Czechoslov. J. Phys., 29(7):810, 1979

[240] J. H. Harris, R. A. Youngman, "Time-resolved luminescence of oxygen related defects in aluminum nitride", Mat. Res. Soc. Symp. Proc., 167:253, 1989

[241] D. Redfield, M. a. Afromowitz, "The direct absorption edge in covalent solids", Appl. Phys. Lett., 11(4):138, 1967

[242] J. H. Harris, R. A. Youngman, R. G. Teller, "On the nature of the oxygenrelated defect in aluminum nitride", J. Mater. Res., 5(08):1763, 1990

[243] J. Neugebauer, C. G. Van de Walle, "Gallium vacancies and the yellow luminescence in GaN", Appl. Phys. Lett., 69(4):503, 1996

[244] M. Cardona, "Optical properties of the silver and cuprous halides", Phys. Rev., 129(1):69, 1963

[245] S. R. Lee, A. F. Wright, M. H. Crawford, G. A. Petersen, J. Han, R. M. Biefeld, "The band-gap bowing of $\mathrm{Al}_{x} \mathrm{Ga}_{1-x} \mathrm{~N}$ alloys", Appl. Phys. Lett., 74(22):3344, 1999

[246] J.-M. Mäki, I. Makkonen, F. Tuomisto, A. Karjalainen, S. Suihkonen, J. Räisänen, T. Y. Chemekova, Y. N. Makarov, "Identification of the $V_{A l}-O_{N}$ defect complex in AlN single crystals", Phys. Rev. B, 84(8):081204, 2011

[247] J. Senawiratne, M. Strassburg, N. Dietz, U. Haboeck, A. Hoffmann, V. Noveski, R. Dalmau, R. Schlesser, Z. Sitar, "Raman, photoluminescence and absorption studies on high quality AlN single crystals", Phys. status solidi, 2(7):2774, 2005

[248] M. Strassburg, J. Senawiratne, N. Dietz, U. Haboeck, A. Hoffmann, V. Noveski, R. Dalmau, R. Schlesser, Z. Sitar, "The growth and optical properties of large, high-quality AlN single crystals", J. Appl. Phys., 96(10):5870, 2004

[249] K. Irmscher, C. Hartmann, C. Guguschev, M. Pietsch, J. Wollweber, M. Bickermann, "Identification of a tri-carbon defect and its relation to the ultraviolet absorption in aluminum nitride", J. Appl. Phys., 114(12):123505, 2013

[250] F. Karel, J. Pastrňák, "Phonon structure of $\mathrm{Mn}^{4+}$ activator centres in AlN", Czechoslov. J. Phys. B, 19:78, 1969 
[251] L. Trinkler, B. Berzina, "Localised transitions in luminescence of AlN ceramics", Radiat. Meas., 71:232, 2014

[252] H. J. Lozykowski, "Kinetics of luminescence of isoelectronic rare-earth ions in III-V semiconductors", Phys. Rev. B, 48(24):17758, 1993

[253] N. S. VanDamme, S. M. Richard, S. R. Winzer, "Liquid-phase sintering of aluminum nitride by europium oxide additives", J. Am. Ceram. Soc., $72(8): 1409,1989$

[254] M. Peres, A. Cruz, M. Soares, A. Neves, T. Monteiro, K. Lorenz, E. Alves, "Optical and structural studies in Eu-implanted AlN films", Superlattices Microstruct., 40(4):537, 2006

[255] K. Lorenz, E. Alves, F. Gloux, P. Ruterana, "RE implantation and annealing of III-nitrides", "Rare-earth doped III-nitrides Optoelectron. Spintron. Appl.", chap. 2, 25-54, 2010

[256] R. Weingärtner, O. Erlenbach, A. Winnacker, A. Welte, I. Brauer, H. Mendel, H. Strunk, C. Ribeiro, A. Zanatta, "Thermal activation, cathodo- and photoluminescence measurements of rare earth doped (Tm,Tb,Dy,Eu,Sm,Yb) amorphous/nanocrystalline AlN thin films prepared by reactive rf-sputtering", Opt. Mater. (Amst)., 28(6):790, 2006

[257] J. Rodrigues, S. Miranda, N. Santos, A. Neves, E. Alves, K. Lorenz, T. Monteiro, "Rare earth co-doping nitride layers for visible light", Mater. Chem. Phys., 134(2):716, 2012

[258] P. Dorenbos, E. van der Kolk, "Location of lanthanide impurity energy levels in the III-V semiconductor $\mathrm{Al}_{x} \mathrm{Ga}_{1-x} \mathrm{~N}(0 \leq x \leq 1)$ ", Opt. Mater. (Amst)., 30(7):1052, 2008

[259] U. Vetter, "Lanthanide doped wide band gap semiconductors : Intra- $4 f$ luminescence and lattice location studies", Ph.D. thesis, 2003

[260] L.-J. Yin, W. Yu, X. Xu, L.-Y. Hao, A. Simeon, "The effects of fluxes on AlN:Eu ${ }^{2+}$ blue phosphors synthesized by a carbothermal reduction method", J. Am. Ceram. Soc., 94(11):3842, 2011

[261] K. Wang, K. P. O'Donnell, B. Hourahine, R. W. Martin, I. M. Watson, K. Lorenz, E. Alves, "Luminescence of Eu ions in $\mathrm{Al}_{x} \mathrm{Ga}_{1-x} \mathrm{~N}$ across the entire alloy composition range", Phys. Rev. B, 80(12):125206, 2009

[262] "Verdi G-Series laser operator's manual", Coherent Inc., 1169144, 2011 
[263] S. a. R. Horsley, J.-H. Wu, M. Artoni, G. C. L. Rocca, "Revisiting the Bragg reflector to illustrate modern developments in optics", Am. J. Phys., 82(3):206, 2014

[264] Iso, "Lasers and laser-related equipment - Test methods for laser beam widths, divergence angles and beam propagation ratios - Stigmatic and simple astigmatic beams ISO 11146-1", 2005

[265] J. Stone, A. R. Chraplyvy, C. A. Burrus, "Gas-in-glass: new way to generate light", Opt. News, 8(6):9, 1982

[266] "MIRA900 - Modelocked Ti:Sapphire lasers", Coherent Inc., MC-135-022.5M0603, 2002

[267] J. Kerr, "A new relation between electricity and light: Dielectrified media birefringent.", Philos. Mag., 50(332):337, 1875

[268] R. Menzel, Photonics - Linear and Nonlinear Interactions of Laser Light and Matter, Springer Berlin Heidelberg New York, 2001

[269] A. Major, F. Yoshino, I. Nikolakakos, J. S. Aitchison, P. W. E. Smith, "Dispersion of the nonlinear refractive index in sapphire", Opt. Lett., 29(6):602, 2004

[270] "Operator's manual - The Coherent Mira model 900-F laser", Coherent Inc., 1997

[271] T. L. Koch, J. E. Bowers, "Nature of wavelength chirping in directly modulated semiconductor lasers", Electron. Lett., 20(25):1038, 1984

[272] B. Lyot, "Un monochromateur à grand champ utilisant les interférences en lumiére polarisée", C. R. Acad. Sci. Paris, 197:1593, 1933

[273] J. P. Peixto, A. H. Oort, "Physics of climate", "Chapter 6 Radiat. Balanc.", 91-130, 1992

[274] J. Eddie H. Young, "Design considerations for acousto-optic devices", Proc. IEEE, 69(1):54, 1981

[275] "Model 9200 pulse picker operator's manual", Coherent Inc., 0165-430-0, 2005

[276] C. S. Zha, R. J. Hemley, H. K. Mao, T. S. Duffy, C. Meade, "Acoustic velocities and refractive index of $\mathrm{SiO} 2$ glass to $57.5 \mathrm{GPa}$ by Brillouin scattering", 1994

[277] K.-i. Kondo, "Nonlinear pressure dependence of the elastic moduli of fused quartz up to 3 GPa", J. Appl. Phys., 52(4):2826, 1981 
[278] A. Savage, R. C. Miller, "Measurements of second harmonic generation of the ruby laser line in piezoelectric crystals", Appl. Opt., 1(5):661, 1962

[279] P. A. Franken, A. E. Hill, C. W. Peters, G. Weinreich, "Generation of optical harmonics", Phys. Rev. Lett., 7(4):118, 1961

[280] J. Soret, E. Sarasin, "On the rotatory polarization of quartz", Philos. Mag. Ser. 5, 2(13):475, 1876

[281] "HarmoniXX Generator (SHG/THG/FHG)", APE, 2010

[282] O. Beck, "Zeitaufgelöste Photolumineszenz-Spektroskopie an Seltenerddotiertem Aluminiumnitrid", Masterarbeit, 2013

[283] "Model SR540 optical chopper", Stanford Res. Syst. Inc., 2009

[284] R. G. Lyons, "Chapter two. Periodic sampling", "Underst. Digit. signal Process.", vol. 3, 43-63, 2010

[285] "Synchronous optical network (SONET) transport systems: common generic criteria", GR-253-CORE, (4), 2005

[286] ITU-T, "G.810 - Definitions and terminology for synchronization networks", G.810, 1997

[287] "Understanding and characterizing timing jitter", Tektronix, 2012

[288] G. Pólya, "Über den zentralen Grenzwertsatz der Wahrscheinlichkeitsrechnung und das Momentenproblem", Math. Zeitschrift, 8(3):171

[289] R. Stephens, "Part 2: What the dual-Dirac model is and what it is not", Jitter 360 Knowl. Ser., (1):1, 2006

[290] "CTI-Cryogenics Cryodyne refrigeration system: Model 22, 350, 1020 and 1050", Cryodyne@ Refrig. Syst., 2002

[291] "Allectra Katalog: Koaxial Durchführungen", Allectra GmbH, 3.1-3.14, 2013

[292] M. Czerny, A. F. Turner, "Über den Astigmatismus bei Spiegelspektrometern", Zeitschrift für Phys., 61(11-12):792, 1930

[293] "Detector components, IGA series photodiode", Eos (Washington. DC).

[294] "510 48 XXX", Horiba Ltd, http//www.horiba.com/uploads/pics/510a486.gif, 15.09.2016

[295] "530 25 XXX", Horiba Ltd, http//www.horiba.com/uploads/pics/530a25\%.gif, 12.10.2016 
[296] Hamamatsu Photonics K.K., "Photomultiplier tubes R928, R955 (TPMS1091E01)", 2016

[297] "Acton series monochromators and spectrographs", Princet. Instruments, Act. Ser. Rev. N3.1

[298] "Grating efficiency curve 50g/mm with 600nm blaze", Newport Corp. http//www.princetoninstruments.com/userfiles/images/products/accessories/ grating-curves/1-005-600-1469-Sept2010.png, 31.05.2016

[299] "Diffraction grating specification sheet", Newport Corp. http//www.gratinglab.com/Products/Product_Tables/Efficiency/Efficiency.asp $x$ ? catalog $=53-{ }^{*}-172 R, 31.05 .2016$

[300] Corporation, "Grating efficiency curve $900 \mathrm{~g} / \mathrm{mm}$ with 550nm blaze", Newport Corp. http//www.princetoninstruments.com/Uploads/Princeton/Images/Gra .../1-090-550-1329-Sept2010.gif, 01.01.2012

[301] C. Palmer, E. Loewen, Diffraction grating handbook, Newport Corporation, 6th ed., 2005

[302] C. J. Sansonetti, M. L. Salit, J. Reader, "Wavelengths of spectral lines in mercury pencil lamps.", Appl. Opt., 35(1):74, 1996

[303] Hamamatsu Photonics K.K., "Universal streak camera C10910 series", 2012

[304] Hamamatsu Photonics K.K., "Universal streak camera C10910 instruction manual", 2012

[305] H. Hertz, "Ueber einen Einfluss des ultravioletten Lichtes auf die electrische Entladung", Ann. Phys., 267(8):983, 1887

[306] A. Einstein, "Über einen die Erzeugung und Verwandlung des Lichtes betreffenden heuristischen Gesichtspunkt", Ann. Phys., 322(6):132, 1905

[307] Hamamatsu Photonics K.K., "Digital CCD camera C10600-10B instruction manual", 2011

[308] M. Peltier, "Nouvelles expériences sur la caloricité des courans électriques", Ann. Chim., 56:371, 1834

[309] Hamamatsu Photonics K.K., "Synchronous delay generator C10647-01 instruction Manual", 2009

[310] Thorlabs Inc., "DET10A(/M) - Si biased detector - user guide", Rev E:1, 2015

[311] Hamamatsu Photonics K.K., "Delay unit C1097-05 instruction manual", 2007 
[312] STMicroelectronics N. V., "UM1472 User manual - Discovery kit for STM32F407/417 lines", 2014

[313] I. Wolfram Research, "Mathematica", 2010

[314] T. Taniguchi, S. Yamaoka, "Spontaneous nucleation of cubic boron nitride single crystal by temperature gradient method under high pressure", J. Cryst. Growth, 222(3):549, 2001

[315] S. Shionoya, T. Koda, K. Era, H. Fujiwara, "Nature of luminescence transitions in ZnS crystals", J. Phys. Soc. Japan, 19(7):1157, 1964

[316] S. N. F. Mott, R. W. Gurney, Electronic processes in ionic crystals, Oxford: Clarendon Press, 2nd ed., 1950

[317] A. S. Davydov, "The theory of molecular excitons (translation)", Sov. Phys. Uspekhi, 82(3-4):393, 1964

[318] M. H. L. Pryce, "Interaction of lattice vibrations with electrons at point defects", "Phonons perfect lattices lattices with point imperfections", 1966, 403-448

[319] T. Williams, C. Kelley, Others, "Gnuplot 4.6: an interactive plotting program", 2016

[320] J. L. Skinner, D. Hsu, "Optical dephasing of ions and molecules in crystals", "Adv. Chem. Physics, Vol. 65", John Wiley \& Sons, Inc., chap. 1, 1-44, 1986

[321] E. D. Nelson, J. Y. Wong, A. L. Schawlow, "Far infrared spectra of $\mathrm{Al}_{2} \mathrm{O}_{3}: \mathrm{Cr}^{3+}$ and $\mathrm{Al}_{2} \mathrm{O}_{3}: \mathrm{Ti}^{3+}$, Phys. Rev., 156(2):298, 1967

[322] D. S. McClure, "Optical spectra of transition-metal ions in corundum", J. Chem. Phys., 36(10):2757, 1962

[323] H. A. Jahn, E. Teller, "Stability of polyatomic molecules in degenerate electronic states. I. Orbital degeneracy", Proc. R. Soc. A Math. Phys. Eng. Sci., 161(905):220, 1937

[324] H. A. Kramers, "Théorie générale de la rotation paramagnétique dans les cristaux", Proc. K. Akad. van Wet., (33):959, 1930

[325] J. Franck, E. G. Dymond, "Elementary processes of photochemical reactions", Trans. Faraday Soc., 21(February):536, 1926

[326] E. Condon, "A theory of intensity distribution in band systems", Phys. Rev., 28(6):1182, 1926 
[327] P. F. Moulton, "Spectroscopic and laser characteristics of Ti: $\mathrm{Al}_{2} \mathrm{O}_{3}$ ", J. Opt. Soc. Am. B, 3(1):125, 1986

[328] B. F. Gächter, "Zero phonon transitions and interacting Jahn-Teller phonon energies from the fluorescence spectrum of $\alpha-\mathrm{Al}_{2} \mathrm{O}_{3}: \mathrm{Ti}^{3+} "$, J. Chem. Phys., 60(5):2003, 1974

[329] M. Manga, R. Jeanloz, "Thermal conductivity of corundum and periclase and implications for the lower mantle", J. Geophys. Res., 102(B2):2999, 1997

[330] R. Armiento, A. E. Mattsson, "Functional designed to include surface effects in self-consistent density functional theory", Phys. Rev. B, 72(8):085108, 2005

[331] O. K. Andersen, "Linear methods in band theory", Phys. Rev. B, 12(8):3060, 1975

[332] A. D. Becke, "Density-functional thermochemistry. III. The role of exact exchange", J. Chem. Phys., 98(7):5648, 1993

[333] P. J. Stephens, F. J. Devlin, C. F. Chabalowski, M. J. Frisch, "Ab Initio calculation of vibrational absorption and circular dichroism spectra using density functional force fields", J. Phys. Chem., 98(45):11623, 1994

[334] E. Engel, S. H. Vosko, "Exact exchange-only potentials and the virial relation as microscopic criteria for generalized gradient approximations", Phys. Rev. $B, 47(20): 13164,1993$

[335] M. Hybertsen, S. Louie, "First-principles theory of quasiparticles: Calculation of band gaps in semiconductors and insulators.", Phys. Rev. Lett., 55(13):1418, 1985

[336] J. M. Luttinger, W. Kohn, "Motion of electrons and holes in perturbed periodic fields", Phys. Rev., 97(4):869, 1955

[337] W. Y. Ching, "Theoretical studies of the electronic properties of ceramic materials", J. Am. Ceram. Soc., 73(11):3135, 1990

[338] L. Ferreira, M. Marques, L. Teles, "Approximation to density functional theory for the calculation of band gaps of semiconductors", Phys. Rev. B, 78(12):125116, 2008

[339] P. E. Blöchl, "Projector augmented-wave method", Phys. Rev. B, 50(24):17953, 1994

[340] J. P. Perdew, K. Burke, M. Ernzerhof, "Generalized gradient approximation made simple", Phys. Rev. Lett., 77(18):3865, 1996 
[341] J. P. Perdew, K. A. Jackson, M. R. Pederson, D. J. Singh, C. Fiolhais, "Atoms, molecules, solids, and surfaces: Applications of the generalized gradient approximation for exchange and correlation", Phys. Rev. B, 46(11):6671, 1992

[342] J. P. Perdew, A. Zunger, "Self-interaction correction to density-functional approximations for many-electron systems", Phys. Rev. B, 23(10):5048, 1981

[343] A. D. Becke, "Density-functional exchange-energy approximation with correct asymptotic behavior", Phys. Rev. A, 38(6):3098, 1988

[344] J. C. Slater, G. F. Koster, "Simplified LCAO method for the periodic potential problem", Phys. Rev., 94(6):1498, 1954

[345] N. E. Christensen, I. Gorczyca, "Optical and structural properties", Phys. Rev. B, 50(7):4397, 1994

[346] M. van Schilfgaarde, a. Sher, a. B. Chen, "Theory of AlN, GaN, InN and their alloys", J. Cryst. Growth, 178(1-2):8, 1997

[347] M. Goano, E. Bellotti, E. Ghillino, G. Ghione, K. F. Brennan, "Band structure nonlocal pseudopotential calculation of the III-nitride wurtzite phase materials system. Part I. Binary compounds GaN, AlN, and InN", J. Appl. Phys., 88(11):6467, 2000

[348] D. Fritsch, H. Schmidt, M. Grundmann, "Band-structure pseudopotential calculation of zinc-blende and wurtzite AlN, GaN, and InN", Phys. Rev. B, $67(23): 235205,2003$

[349] R. Ahmed, H. Akbarzadeh, "A first principle study of band structure of III-nitride compounds", Phys. B Condens. Matter, 370(1-4):52, 2005

[350] C. Persson, A. Ferreira da Silva, R. Ahuja, B. Johansson, "Effective electronic masses in wurtzite and zinc-blende GaN and AlN", J. Cryst. Growth, 231(3):397, 2001

[351] M. Suzuki, T. Uenoyama, A. Yanase, "First-principles calculations of effectivemass parameters of AlN and GaN", Phys. Rev. B, 52(11):8132, 1995

[352] F. Litimein, B. Bouhafs, Z. Dridi, P. Ruterana, "The electronic structure of wurtzite and zincblende AlN: an ab initio comparative study", New J. Phys., $4(1): 64.1,2002$

[353] A. Ferreira da Silva, N. Souza Dantas, J. de Almeida, R. Ahuja, C. Persson, "Electronic and optical properties of wurtzite and zinc-blende TIN and AlN", J. Cryst. Growth, 281(1):151, 2005 
[354] N. E. Christensen, I. Gorczyca, "Calculated structural phase transitions of aluminum nitride under pressure", Phys. Rev. B, 47(8):4307, 1993

[355] A. Rubio, J. L. Corkill, M. L. Cohen, E. L. Shirley, S. G. Louie, "Quasiparticle band structure of AlN and GaN", Phys. Rev. B, 48(16):11810, 1993

[356] M. M. Armenta, A. Reyes-Serrato, M. Avalos Borja, "Ab initio determination of the electronic structure of beryllium-, aluminum-, and magnesium-nitrides: A comparative study", Phys. Rev. B, 62(8):4890, 2000

[357] J. A. Majewski, M. Städele, P. Vogl, "Electronic structure of biaxially-strained wurtzite crystals GaN and AlN", MRS Proc., 449:887, 1997

[358] D. Dugdale, S. Brand, R. Abram, "Direct calculation of k.p parameters for wurtzite AlN, GaN and InN.", 61(19):933, 2000

[359] M.-Z. Huang, W. Ching, "A minimal basis semi-ab initio approach to the band structures of semiconductors", J. Phys. Chem. Solids, 46(8):977, 1985

[360] W. Ching, B. N. Harmon, "Electronic structure of AlN", 34(8):5305, 1986

[361] S. Loughin, R. H. French, W. Y. Ching, Y. N. Xu, G. A. Slack, "Electronic structure of aluminum nitride: Theory and experiment", Appl. Phys. Lett., 63(9):1182, 1993

[362] Y.-N. Xu, W. Ching, "Electronic, optical, and structural properties of some wurtzite crystals", Phys. Rev. B, 48(7):4335, 1993

[363] D. Vogel, P. Krüger, J. Pollmann, "Structural and electronic properties of group-III nitrides", Phys. Rev. B, 55(19):12836, 1997

[364] C. Stampfl, C. Van de Walle, "Density-functional calculations for III-V nitrides using the local-density approximation and the generalized gradient approximation", Phys. Rev. B, 59(8):5521, 1999

[365] P. van Camp, V. van Doren, J. Devreese, "High-pressure properties of wurtziteand rocksalt-type aluminum nitride", Phys. Rev. B, 44(16):9056, 1991

[366] K. Miwa, A. Fukumoto, "First-principles calculation of the structural, electronic, and vibrational properties of gallium nitride and aluminum nitride", Phys. Rev. B, 48(11):7897, 1993

[367] A. F. Wright, J. S. Nelson, "Consistent structural properties for AlN, GaN, and InN", 51(12):7866, 1995

[368] K. Shimada, T. Sota, K. Suzuki, "First-principles study on electronic and elastic properties of BN, AlN, and GaN", J. Appl. Phys., 84(9):4951, 1998 
[369] J.-M. Wagner, F. Bechstedt, "Properties of strained wurtzite GaN and AlN: Ab initio studies", Phys. Rev. B, 66(11):115202, 2002

[370] B. Rezaei, A. Asgari, M. Kalafi, "Electronic band structure pseudopotential calculation of wurtzite III-nitride materials", Phys. B Condens. Matter, $371(1): 107,2006$

[371] A. Kobayashi, O. F. Sankey, S. M. Volz, J. D. Dow, "Semiempirical tightbinding band structures of wurtzite semiconductors: AlN, CdS, CdSe, ZnS, and ZnO", Phys. Rev. B, 28(2):935, 1983 


\section{A Anhang}

\section{A.1 Titan-Saphir-Laser}

Das $\mathrm{Ti}^{3+}$-Ion ersetzt hierbei im Wirtskristall ein $A l^{3+}$. Dieser Ladungszustand des Titan besitzt lediglich ein einzelnes $3 d$-Elektron außerhalb einer geschlossenen Argonschale, welches im Falle eines freien Ions in einem 10 -fach entarteten ${ }^{2} D$ Zustand vorliegt [321]. Das Kristallfeld, welches das $T i^{3+}$-Ion umgibt, lässt sich in einen starken kubischen, skalierend mit vierter Potenz im Abstand, und einen weitaus schwächeren trigonalen Anteil, skalierend mit zweiter und vierter Potenz, aufteilen [322]. Das kubische Feld allein spaltet das ${ }^{2} D$-Level in ein 4-fach degeneriertes ${ }^{2} E_{g}$ sowie ein darunter liegendes 6-fach degeneriertes ${ }^{2} T_{2 g}$-Level auf [321]. Die Größe dieser Aufspaltung beträgt etwa $2.4 \mathrm{eV}$ [322]. Der trigonale Anteil hingegen spaltet noch einmal das ${ }^{2} T_{2 g}$-Level in ein doppelt degeneriertes ${ }^{2} A_{1}$ und ein 4 -fach degeneriertes ${ }^{2} E$-Niveau auf, nicht jedoch das obere ${ }^{2} E$. Aufgrund der Spin-BahnWechselwirkung wird auch noch das Grundniveau ${ }^{2} E$ in zwei doppelt entartete Level aufgespalten, womit sich die energetischen Unterschiede auf etwa $5 \mathrm{meV}$ zwischen diesen beiden bzw. $13 \mathrm{meV}$ zwischen dem unteren und dem ${ }^{2} A_{1}$-Level belaufen [321]. Der angeregte obere ${ }^{2} E$-Zustand kann sich aufgrund des Jahn-Teller-Effekts 323. und der damit verbundenen örtlichen Neukonfiguration in zwei doppelt degenerierte Niveaus im Abstand von etwa $230 \mathrm{meV}$ aufteilen [322]. Diese nun für alle fünf Level vorherrschende doppelte Entartung (Kramers-Dublett) lässt sich nur durch ein magnetisches Feld aufheben [324]. Eine zusammenfassende Darstellung der Energie-Niveaus des $\mathrm{Ti}^{3+}$-Ion in $\mathrm{Al}_{2} \mathrm{O}_{3}$ ist in Abbildung A.1.(b) gegeben.

Durch optische Anregung kann das 3d-Elektron in einen seiner höheren Zustände gelangen. Nach dem Franck-Condon-Prinzip erfolgt solch ein elektronischer Übergang weitaus schneller als die Relaxation der Atomrümpfe $\left(10^{-15} \mathrm{~s}\right.$ gegenüber $\left.10^{-12} \mathrm{~s}\right)$ [325, 326. Hierbei gibt es endliche Übergangswahrscheinlichkeiten in die verschiedenen vibronischen Niveaus des angeregten bzw. Grundzustands, was zu breiten Absorptions- als auch Emissionsbändern führt. Im angeregten Niveau angekommen, relaxiert das Atom zu einer energetisch günstigeren Position (Jahn-Teller-Effekt [323]) unter Abgabe von Phononen. Bei diesem angeregten Zustand im ${ }^{2} E_{g}$-Level handelt es sich um ein metastabiles Niveau mit einer Lebensdauer von $\tau \approx 3.15(5) \mu$ s bei Raumtemperatur [327]. Ausgehend von dort erfolgt auch hier wieder typischerweise ein vibronischer Übergang zurück auf das ${ }^{2} T_{2}$-Level mit anschließender Relaxation in die alte Gleichgewichtslage (siehe zusammenfassend dazu Abbildung A.1(a)). 


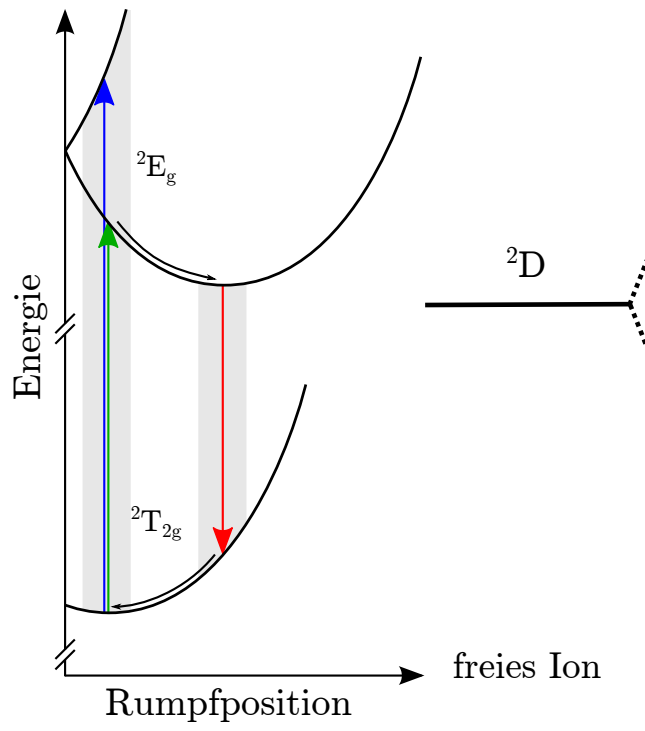

(a)

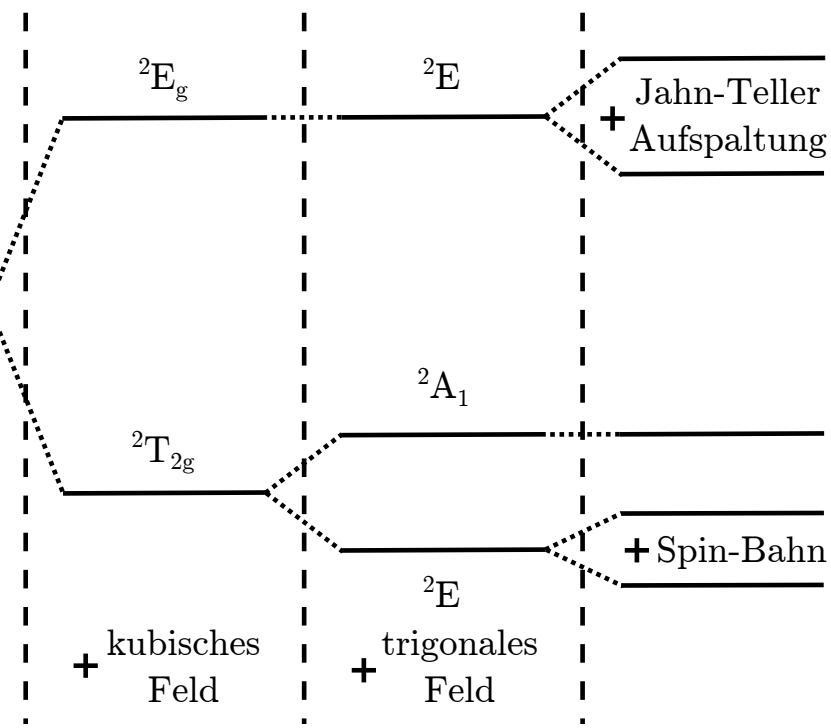

(b)

Abbildung A.1: (a) Schematische Darstellung des Konfigurations-KoordinatenDiagramms für das $3 d$-Elektron des $T i^{3+}$-Ion mit möglicher An- und Abregung. (b) Aufspaltung der Energieniveaus des ${ }^{2} D$-Zustandes unter Berücksichtigung verschiedener Effekte. Die resultierenden Niveaus rechts sind weiterhin doppelt entartet.

Das $\mathrm{Ti}: \mathrm{Al}_{2} \mathrm{O}_{3}$-System zeigt hierbei ein breites Absorptionsspektrum zwischen 400 $600 \mathrm{~nm}$ [322, 327], sowie ein stark Stokes-verschobenes Fluoreszenzspektrum im Bereich von 650 - $1000 \mathrm{~nm}$ 327,328. Aufgrund dieser breiten Emissionsbande können $\mathrm{Ti}: \mathrm{Al}_{2} \mathrm{O}_{3}$-Systeme in einem weiten Bereich von mehreren hundert Nanometer durchgestimmt werden [327]. Durch das relativ langlebige angeregte metastabile Zwischenniveau kann mit genügend Pumpleistung im Bereich um $500 \mathrm{~nm}$ eine hohe Besetzungsinversion, welche für die induzierte Emission benötigt wird, erreicht werden. Weiterhin zeichnet sich Saphir auch durch eine hohe Wärmeleitfähigkeit (>30 W/mK bei Raumtemperatur [329]) aus, wodurch das Material auch hohe Pumpleistungen bei entsprechender Kühlung zerstörungsfrei überstehen kann. 
A.1 Titan-Saphir-Laser

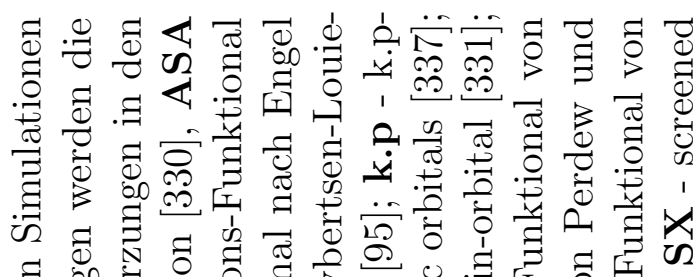

क्ञ

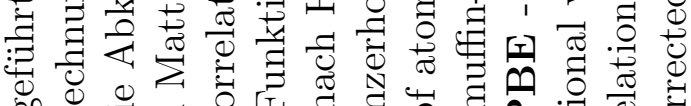

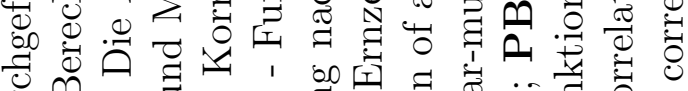

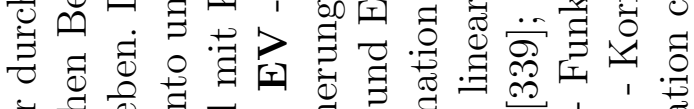

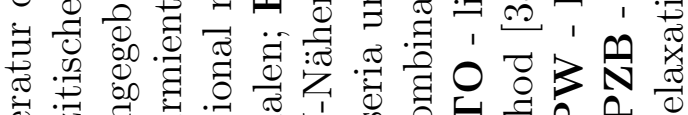

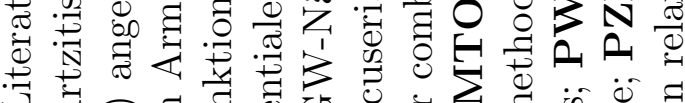

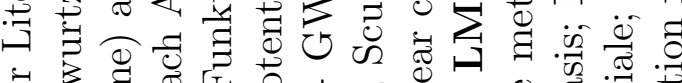

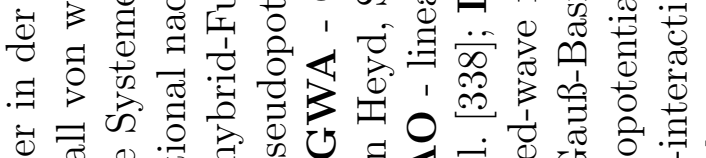

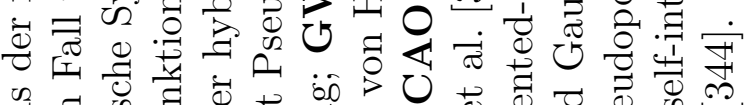

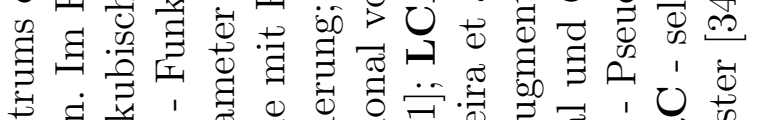

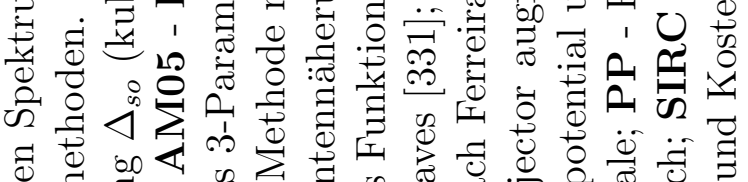

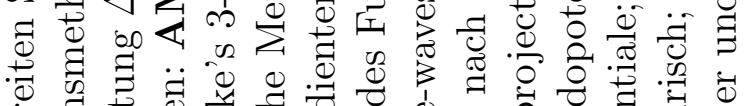

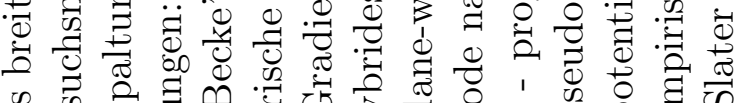
\& .

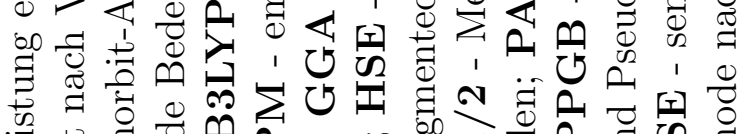

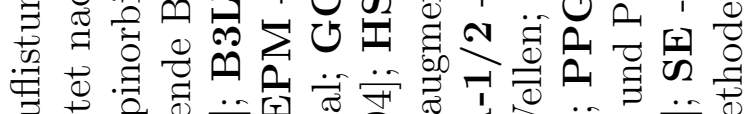

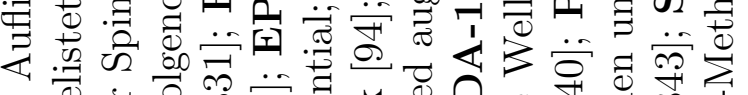

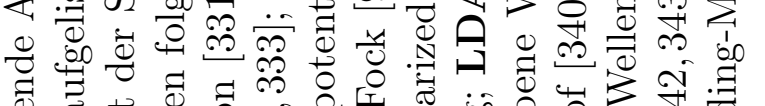

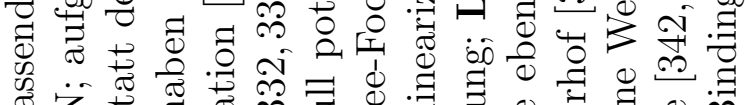

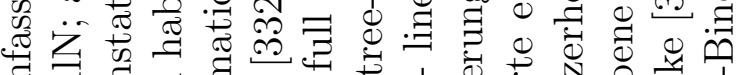

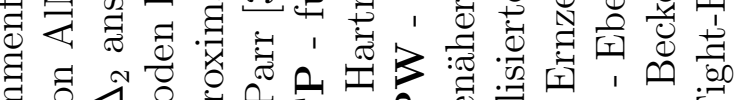

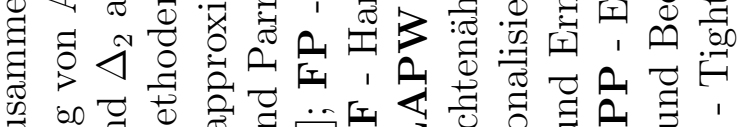
敢

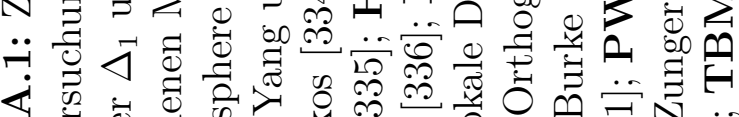

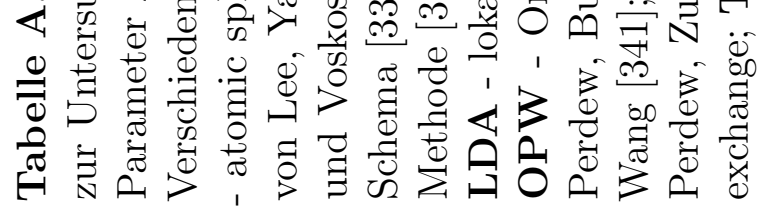

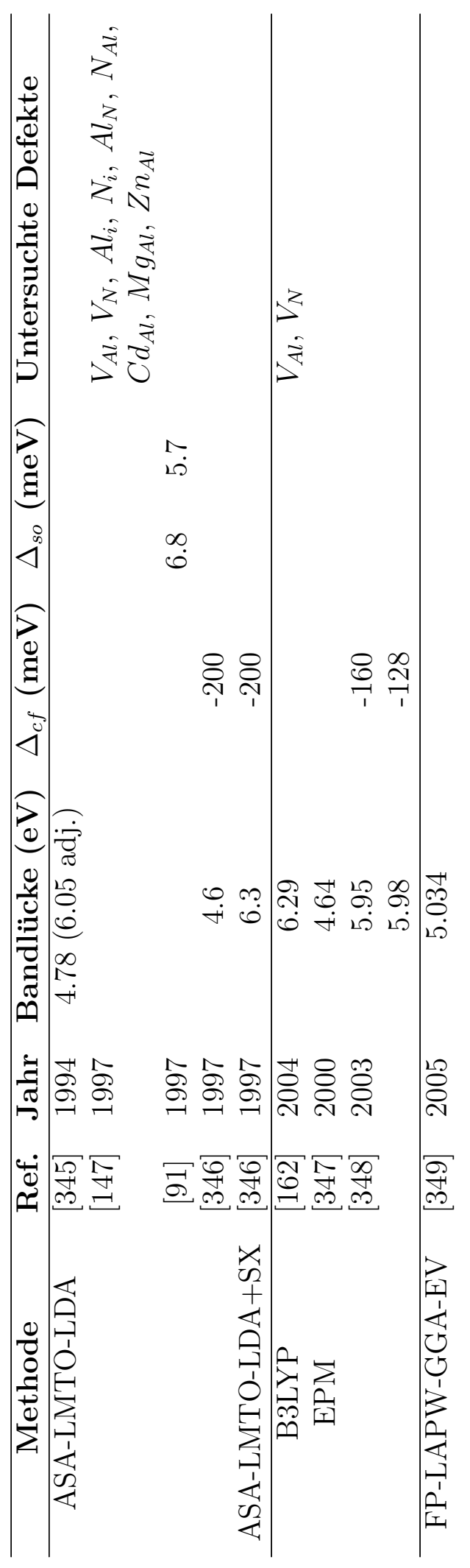




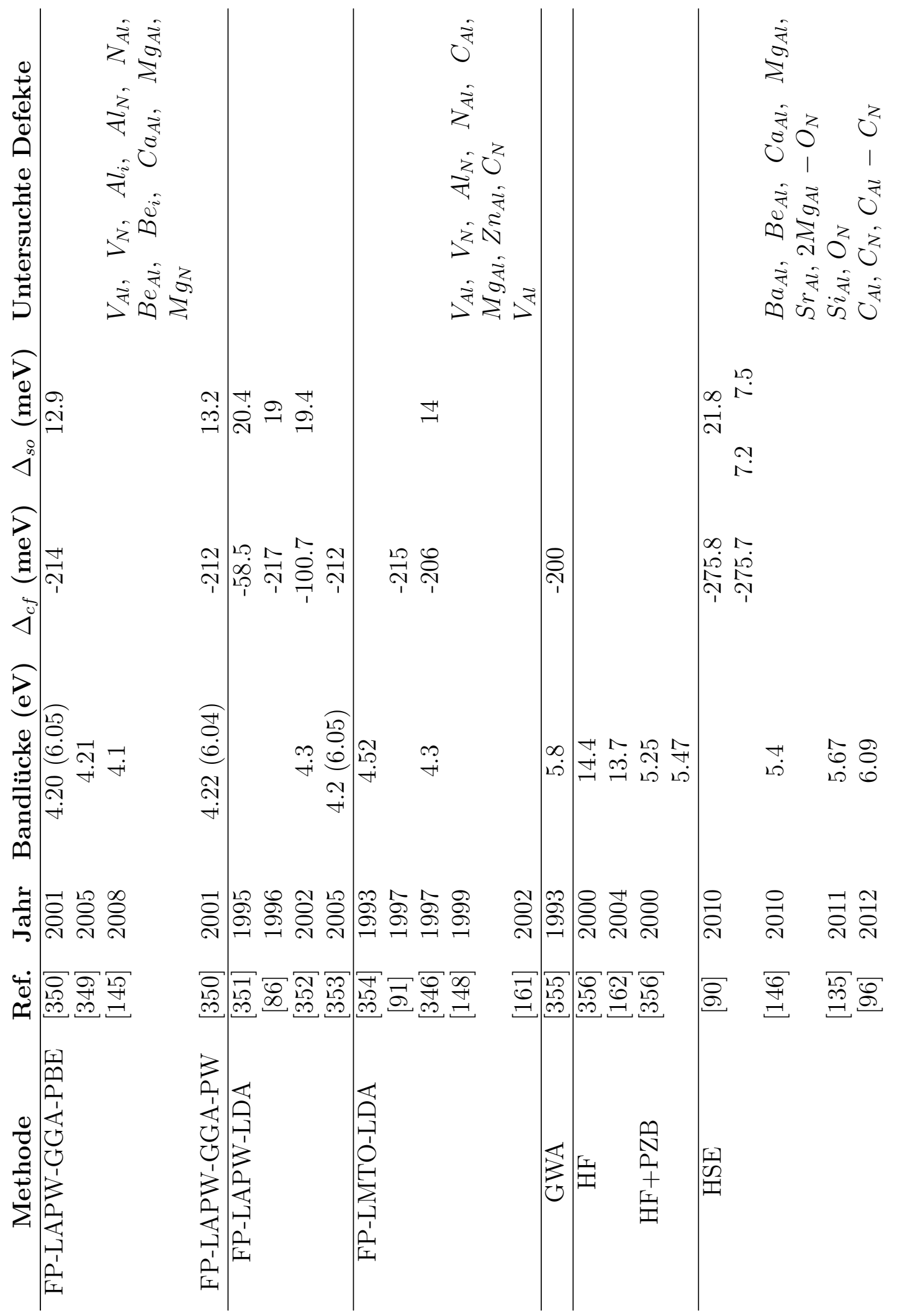




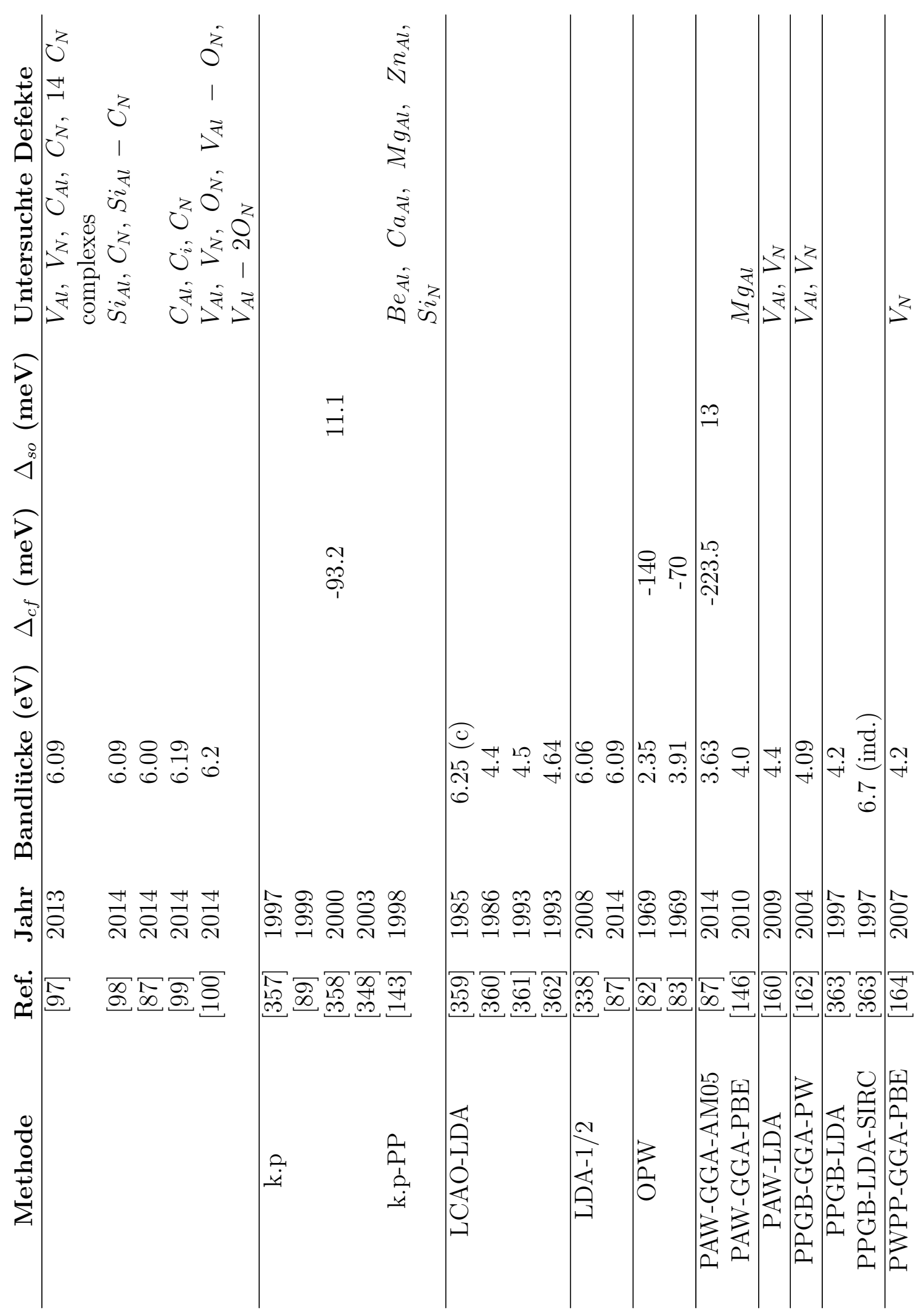




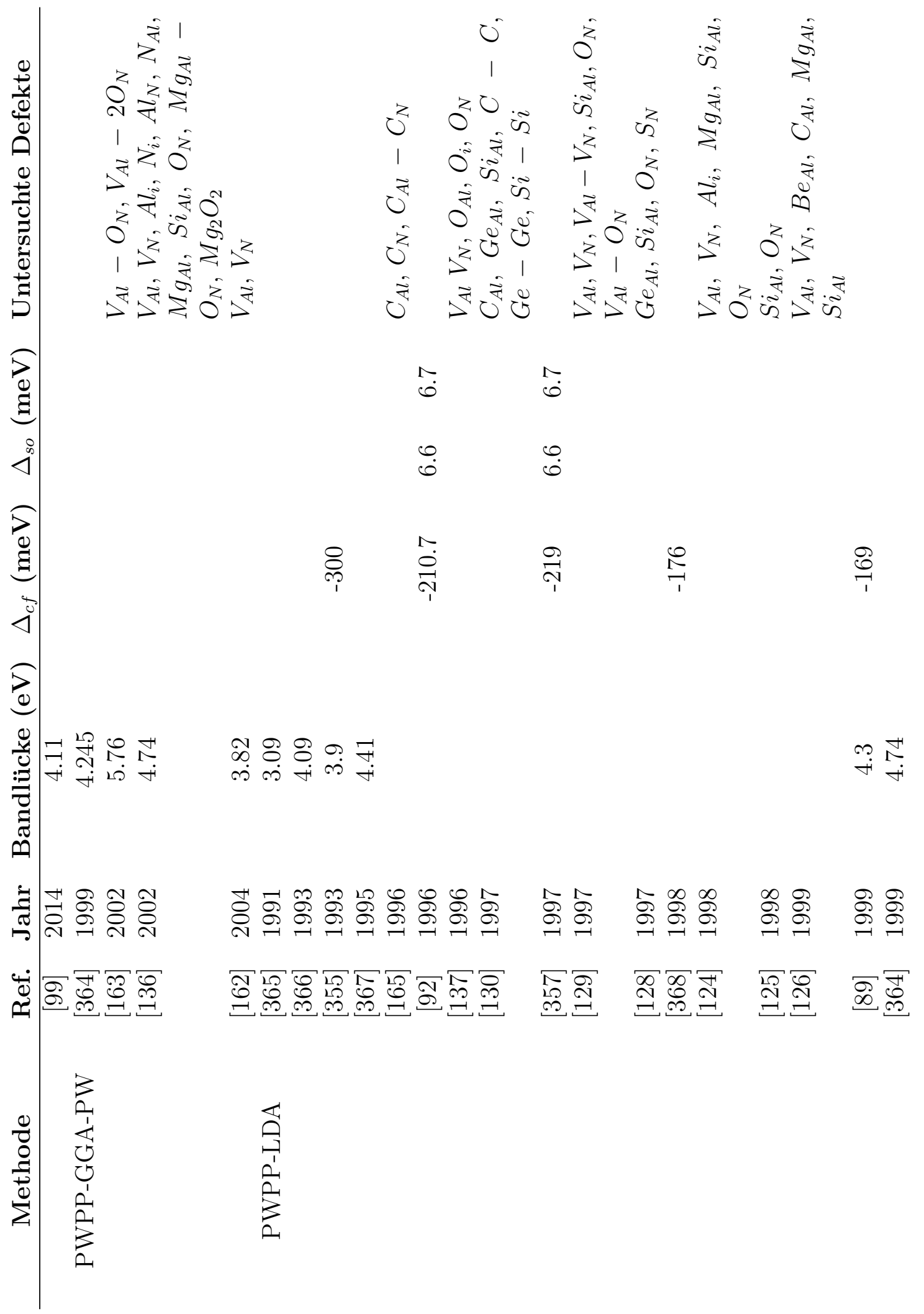




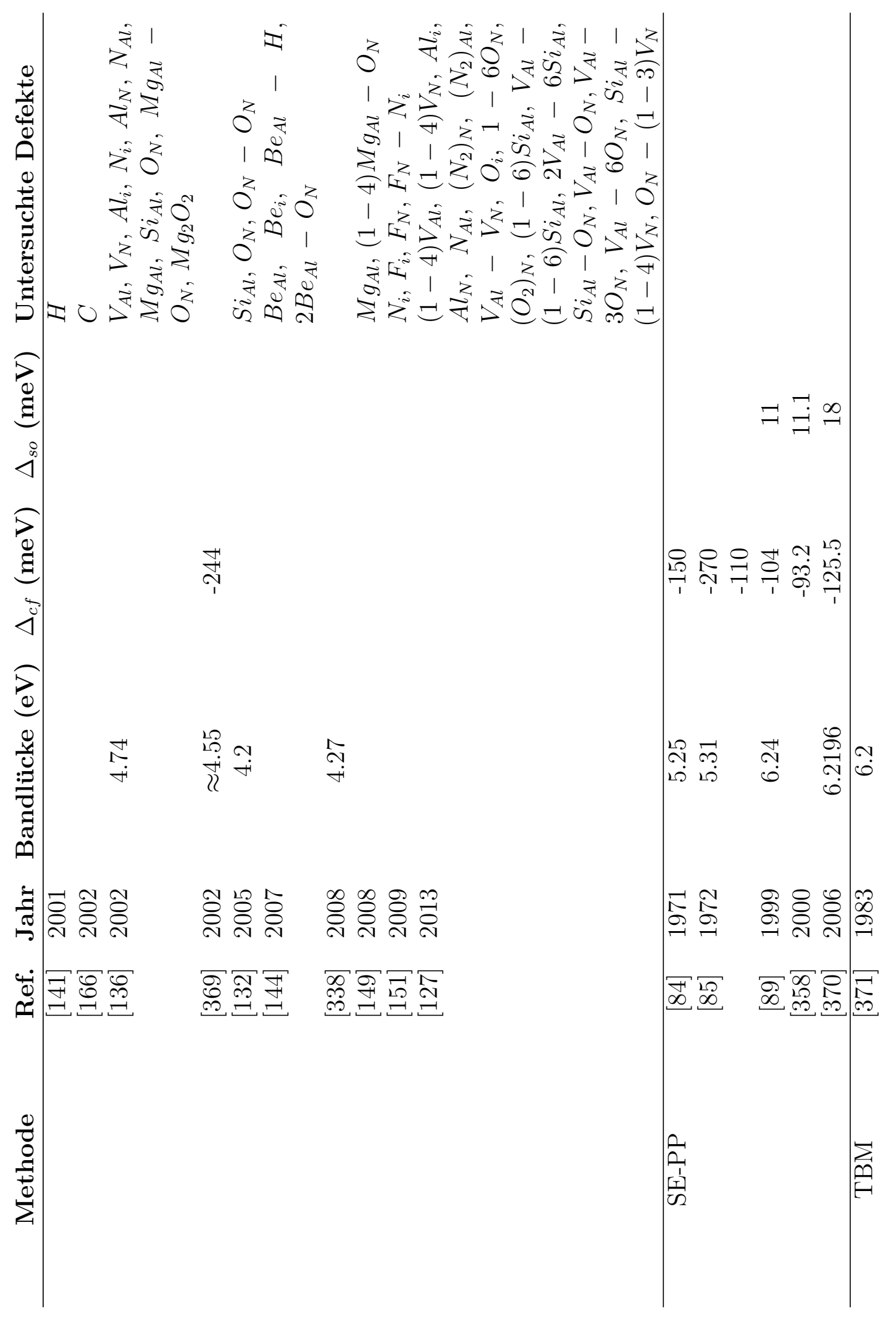




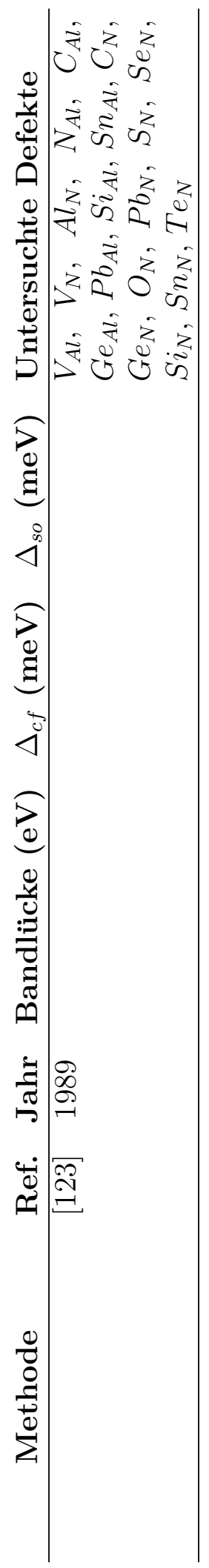


Abfall bei deltaförmiger Pulsanregung

$I_{A}(t)= \begin{cases}0 & \mid t<0 \\ A_{0} \exp \left(-k_{A} t\right) & \mid t \geq 0\end{cases}$

Abfall bei deltaförmiger Pulsanregung mit Zwischenniveau

$I_{B}(t)= \begin{cases}0 & \mid t<0 \\ \frac{k_{B} A_{0}}{k_{A}-k_{B}}\left[\exp \left(-k_{B} t\right)-\exp \left(-k_{A} t\right)\right] & \mid t \geq 0 \wedge k_{A} \neq k_{B} \\ A_{0} k_{B} t \exp \left(-k_{B} t\right) & \mid t \geq 0 \wedge k_{A}=k_{B}\end{cases}$

Abfall mit normalverteiltem Anregungspuls

$I_{A}(t)=\frac{p_{0}}{2}\left[\exp \left(\frac{k_{A}^{2} \sigma^{2}}{2}-k_{A} t\right) \operatorname{erfc}\left(\frac{k_{A} \sigma^{2}-t}{\sqrt{2} \sigma}\right)\right]$

Anstieg bei Anregung durch Stufenfunktion

$I_{A}(t)= \begin{cases}0 & t<0 \\ p_{0}\left[1-\exp \left(-k_{A} t\right)\right] & \mid t \geq 0\end{cases}$

Anstieg bei Anregung durch Stufenfunktion mit Zwischenniveau

$$
I_{B}(t)=\left\{\begin{array}{cl}
0 & \mid t<0 \\
\frac{p_{0}}{k_{A}-k_{B}}\left[\begin{array}{l}
k_{A}\left\{1-\exp \left(-k_{B} t\right)\right\} \\
\left.-k_{B}\left\{1-\exp \left(-k_{A} t\right)\right\}\right]
\end{array}\right. & \mid t \geq 0 \wedge k_{A} \neq k_{B} \\
p_{0}\left[1-\exp \left(-k_{B} t\right)-k_{B} t \exp \left(-k_{B} t\right)\right] & \mid t \geq 0 \wedge k_{A}=k_{B}
\end{array}\right.
$$


Anstieg bei Anregung mit Normalverteilung gefalteter Stufenfunktion

$I_{A}(t)=\frac{p_{0}}{2}\left[\operatorname{erfc}\left(-\frac{t}{\sqrt{2} \sigma}\right)-\exp \left(\frac{k_{A}^{2} \sigma^{2}}{2}-k_{A} t\right) \operatorname{erfc}\left(\frac{k_{A} \sigma^{2}-t}{\sqrt{2} \sigma}\right)\right]$

Abfall bei vorheriger Anregung durch Stufenfunktion

$I_{A}(t)= \begin{cases}p_{0} & \mid t<0 \\ p_{0} \exp \left(-k_{A} t\right) & \mid t \geq 0\end{cases}$

Abfall bei vorheriger Anregung durch Stufenfunktion mit Zwischenniveau

$I_{B}(t)= \begin{cases}p_{0} & \mid t<0 \\ \frac{p_{0}}{k_{A}-k_{B}}\left[k_{A} \exp \left(-k_{B} t\right)-k_{B} \exp \left(-k_{A} t\right)\right] & \mid t \geq 0 \wedge k_{A} \neq k_{B} \\ p_{0}\left[\exp \left(-k_{B} t\right)+k_{B} t \exp \left(-k_{B} t\right)\right] & \mid t \geq 0 \wedge k_{A}=k_{B}\end{cases}$

Abfall bei vorheriger Anregung mit Normalverteilung gefalteter Stufenfunktion

$I_{A}(t)=\frac{p_{0}}{2}\left[\operatorname{erfc}\left(\frac{t}{\sqrt{2} \sigma}\right)+\exp \left(\frac{k_{A}^{2} \sigma^{2}}{2}-k_{A} t\right) \operatorname{erfc}\left(\frac{k_{A} \sigma^{2}-t}{\sqrt{2} \sigma}\right)\right]$

Anstieg und Abfall durch Anregung durch Rechteckfunktion

$I_{A}(t)= \begin{cases}0 & \mid t<-t_{A} \\ p_{0}\left[1-\exp \left(-k_{A}\left\{t+t_{A}\right\}\right)\right] & \mid-t_{A}<t<0 \\ p_{0}\left[1-\exp \left(-k_{A} t_{A}\right)\right] \exp \left(-k_{A} t\right) & \mid t \geq 0\end{cases}$ 
Anstieg und Abfall durch Anregung durch Rechteck mit Zwischenniveau

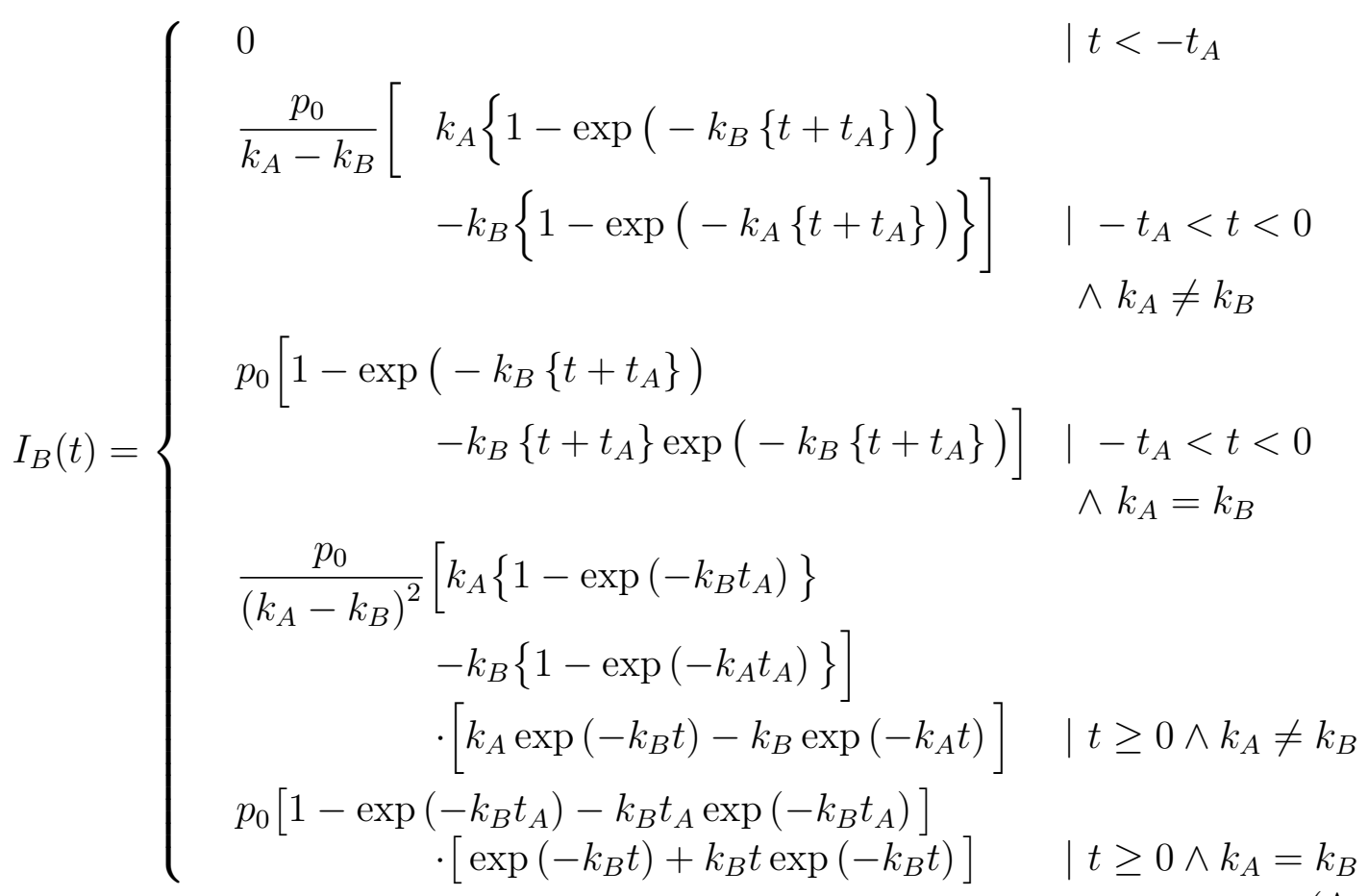

Anstieg und Abfall durch Anregung mit Normalverteilung gefaltetem Rechteck

$$
\begin{aligned}
I_{A}(t)=\frac{p_{0}}{2}[\quad & \operatorname{erfc}\left(\frac{t}{\sqrt{2} \sigma}\right)-\operatorname{erfc}\left(\frac{t_{A}+t}{\sqrt{2} \sigma}\right) \\
& +\exp \left(\frac{k_{A}^{2} \sigma^{2}}{2}-k_{A} t_{A}-k_{A} t\right) \\
& \left.\cdot\left\{\exp \left(k_{A} t_{A}\right) \operatorname{erfc}\left(\frac{k_{A} \sigma^{2}-t}{\sqrt{2} \sigma}\right)-\operatorname{erfc}\left(\frac{k_{A} \sigma^{2}-t_{A}-t}{\sqrt{2} \sigma}\right)\right\}\right]
\end{aligned}
$$


Zerfall nach Pulsanregung und Kaskade in Abbildung 4.11 (c)

$$
\begin{aligned}
I_{B}(t) & =\frac{A_{0} k_{A} k_{B 1}}{k_{B 1}+k_{B 2}-k_{A}}\left[\exp \left(-k_{A} t\right)-\exp \left(-\left\{k_{B 1}+k_{B 2}\right\} t\right)\right] \\
I_{C}(t) & =\frac{A_{0} k_{A} k_{B 2} k_{C}}{k_{B 1}+k_{B 2}-k_{A}}\left[\frac{\exp \left(-\left\{k_{B 1}+k_{B 2}\right\} t\right)}{k_{B 1}+k_{B 2}-k_{C}}-\frac{\exp \left(-k_{A} t\right)}{k_{A}-k_{C}}\right. \\
& \left.-\left(\frac{1}{k_{B 1}+k_{B 2}-k_{C}}-\frac{1}{k_{A}-k_{C}}\right) \exp \left(-k_{C} t\right)\right]
\end{aligned}
$$

Verschiebung und Verbreiterung von Peaks durch Elektron-Phonon-Wechselwirkung nach [320] für pseudo-lokale Phononen mit scharfer Verteilungsfunktion

$$
\begin{aligned}
& \Gamma_{0}(\omega)= \begin{cases}0 & \mid \omega<0 \\
\frac{\omega}{4 \tau_{0}} & \mid \omega \geq 0\end{cases} \\
& \Omega_{0}(\omega)=\frac{1}{2}\left[\frac{\frac{\omega}{\omega_{0}}-1-\left(\frac{\omega_{0} \tau_{0}}{2}\right)^{2}}{\left(\frac{\omega}{\omega_{0}}-1\right)^{2}+\left(\frac{\omega_{0} \tau_{0}}{2}\right)^{2}}\right] \\
& n(\omega, T)=\frac{1}{\exp \left[\frac{\hbar \omega}{k_{\mathrm{B}} T}\right]-1} \\
& E_{0}(T)=\int_{0}^{\infty} \mathrm{d} \omega \frac{1}{2 \pi} \arctan \left[\frac{2 n(\omega, T) W \Gamma_{0}(\omega)\left[1-W \Omega_{0}(\omega)\right]}{\left[1-W \Omega_{0}(\omega)\right]^{2}+[2 n(\omega, T)+1] W^{2} \Gamma_{0}(\omega)^{2}}\right]+E_{0}(0) \\
& \gamma_{0}(T)=\int_{0}^{\infty} \mathrm{d} \omega \frac{1}{4 \pi} \ln \left[1+\frac{4 n(\omega, T)[n(\omega, T)+1] W^{2} \Gamma_{0}(\omega)^{2}}{\left[1-W \Omega_{0}(\omega)\right]^{2}+W^{2} \Gamma_{0}(\omega)^{2}}\right]+\gamma_{0}(0)
\end{aligned}
$$




\title{
Lebenslauf
}

\section{Persönliche Daten}

Name:

Tristan Koppe

Geburtsdatum / -ort: 23.05.1988 / Mühlhausen

Staatsangehörigkeit: deutsch

Familienstand: ledig

\begin{abstract}
Abitur
09/1998 - 06/2006 Gymnasium Oberdorla, 99986 Oberdorla

Albert-Schweitzer-Gymnasium, 99974 Mühlhausen
\end{abstract}

\section{Studium}

10/2006 - 09/2009 Bachelorstudium Physik

Georg-August-Universität Göttingen, 37077 Göttingen

Thema: Verbesserung der elektronischen Auslese von

Photoelektronenvervielfachern für den

Bachelorpraktikumsversuch E4 (Kamiokanne)

Abschluss: Bachelor of Science

10/2009 - 04/2012 Masterstudium Physik

Georg-August-Universität Göttingen, 37077 Göttingen

Thema: FEM-Simulationen und experimentelle

Realisierung von SAW-Strukturen auf $2 \mathrm{H}-\mathrm{AlN}$

Abschluss: Master of Science 